\section{Check for updates}

Cite this: Chem. Soc. Rev., 2021, 50, 11098

Received 20th April 2021

DOI: $10.1039 / \mathrm{d} 1 \mathrm{cs} 00271 \mathrm{f}$

rsc.li/chem-soc-rev

\title{
Cysteine protecting groups: applications in peptide and protein science
}

\author{
Richard J. Spears, ${ }^{*}$ Clíona McMahon and Vijay Chudasama (D) *
}

\begin{abstract}
Protecting group chemistry for the cysteine thiol group has enabled a vast array of peptide and protein chemistry over the last several decades. Increasingly sophisticated strategies for the protection, and subsequent deprotection, of cysteine have been developed, facilitating synthesis of complex disulfiderich peptides, semisynthesis of proteins, and peptide/protein labelling in vitro and in vivo. In this review, we analyse and discuss the $60+$ individual protecting groups reported for cysteine, highlighting their applications in peptide synthesis and protein science.
\end{abstract}

\section{Introduction}

The concept of protecting, and subsequently deprotecting, functional groups is of paramount importance in synthetic chemistry. ${ }^{1-3}$ Protecting group strategies in particular feature extensively in the synthesis of peptides, whereby amino acid building blocks are coupled to one another via amide bonds. This is unsurprising, given the array of functional groups that are found within the 20 proteinogenic amino acids, including: amines, alcohols, thioethers, imidazole rings, and carboxylic acids. ${ }^{4}$ Of the proteinogenic amino acids, cysteine (Cys) has garnered enormous interest within the field of peptide (and protein) chemistry and biology, owing to the unique properties

Department of Chemistry, University College London, London, UK.

E-mail:r.spears@ucl.ac.uk,v.chudasama@ucl.ac.uk

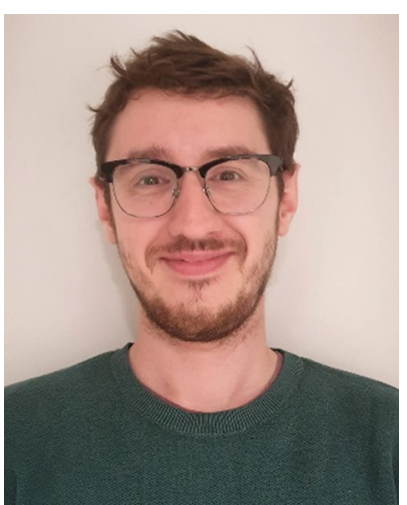

Richard J. Spears
Richard J. Spears received his MChem degree in Chemistry from The University of York in 2014. He then obtained his PhD in Chemistry from The University of York in 2018 under the supervision of Dr Martin A. Fascione, where his work focused on developing new methodologies for site-selective protein modification. In 2018 he joined Prof. Vijay Chudasama's lab as a postdoctoral researcher within University College London, where he currently works on developing novel methodology for use in peptide chemistry and protein bioconjugation. of its thiol side chain. For example, disulfide bonds, which can be formed via the covalent linkage of two Cys thiol side chains, are of utmost importance in defining certain peptides and proteins conformational, proteolytic, chemical, and biophysical properties. ${ }^{5,6}$ Disulfide-containing peptides have also gained huge interest as therapeutic agents. $^{7-9}$ In 1953 Vigneaud, Ressler, Swan, Roberts, Katsoyannis, and Gordon, demonstrated the chemical synthesis of the hormone oxytocin, a disulfide containing peptide that is the focus of a great deal of therapeutic research (Fig. 1a). ${ }^{10,11}$ Vigneaud was later awarded the Nobel prize in Chemistry in 1955. The hormone insulin, which is produced in the pancreas and contains one intrachain and two interchain disulfide bonds, has been a critical component of treating Type I diabetes over the last 100 years (Fig. 1b) ${ }^{12}$ Disulfide-containing peptides also feature in a number of Food and Drug Administration (FDA) approved therapeutics, such as Ziconotide (Prialt ${ }^{\mathrm{TM}}$, a disulfide-rich peptide

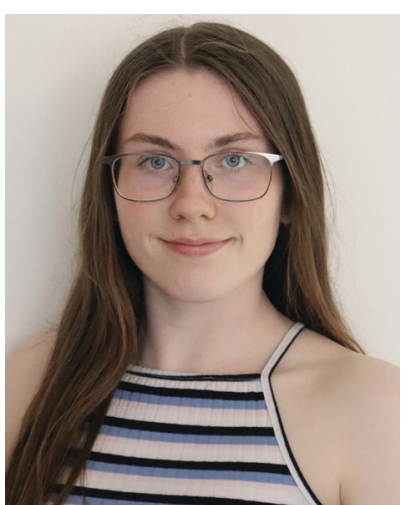

Clíona McMahon
Cliona McMahon obtained her MSci degree in Natural Sciences at UCL, majoring in synthetic organic chemistry and minoring in cell and molecular biology. Her master's research project focused on the development of a cysteine protecting group for use in solid phase peptide synthesis. She will begin a PhD in Chemical Biology in September 2021 in the Chudasama Group, working in the areas of protein and peptide modification. 
for treating severe chronic pain, Fig. 1c), ${ }^{13}$ Linaclotide (Linzess ${ }^{\mathrm{TM}}$, a disulfide-rich peptide for treating severe irritable bowel syndrome), ${ }^{14}$-and ${ }^{68}$ Ga-DOTATOC (Fig. 1d, an octreotide-based tumour imaging agent). ${ }^{15}$ In biology, Cys often finds itself of critical importance in redox processes, ${ }^{16}$ and in the active sites of enzymes such as cysteine proteases. ${ }^{17}$ Cys is also often the site of choice when it comes to the site-specific modification of proteins, also known as bioconjugation, owing to its favourable properties (nucleophilic profile of the thiol at neutral/nearneutral $\mathrm{pH}$, low natural abundance, general ease of incorporation into proteins via site-directed mutagenesis). ${ }^{18}$ This growing field in chemical biology has led to the development of a range of therapeutically relevant bioconjugates, most notable of which include antibody-drug conjugates (ADCs); ${ }^{19}$ these include FDA approved Adcetris ${ }^{\mathrm{TM}}$ (Fig. 1e, brentuximab vedotin, used to treat lymphoma), Polivy ${ }^{\mathrm{TM}}$ (polatuzumab vedotin, used to treat lymphoma), and Padcev ${ }^{\mathrm{TM}}$ (enfortumab vedotin, used to treat urothelial cancer), all of which have their therapeutic payload covalently attached to the antibody via Cys conjugation. ${ }^{20}$

The reactivity profile of Cys enables a plethora of applications in chemistry and biology related to peptides and proteins. However, this reactivity also leads to significant challenges when employing the residue for peptide chemistry in particular. The reactive nature of the thiol side chain makes it prone to side reactions such as alkylation and oxidation. ${ }^{21}$ Particularly for disulfide-rich peptides, ensuring the regioselective formation/ correct connectivity of disulfides presents a significant challenge in peptide synthesis. ${ }^{5,6}$ Ensuring racemisation of Cys at the $\alpha$-carbon stereocenter does not occur during peptide synthesis also presents a notable challenge; this is especially true for synthesising peptides containing a C-terminal Cys. ${ }^{22}$ Other side reactions related to Fmoc SPPS include formation of 3-(1-piperidinyl)alanine by-products at the C-terminal Cys position formed by an elimination-addition reaction involving piperidine. ${ }^{23,24}$ Unsurprisingly, this has led to significant research into strategies to

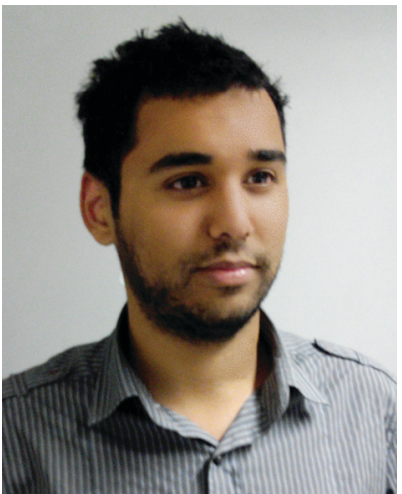

Vijay Chudasama
Prof. Vijay Chudasama obtained his MSci degree and PhD from University College London (UCL) in 2008 and 2011, respectively. Following post-doctoral studies under the supervision of Prof. Stephen Caddick, Vijay obtained a Ramsay Memorial Fellowship. During this time, he was made Technical Director of a biotechnology spin-out (ThioLogics). In April 2015, he was appointed as a Lecturer at UCL Department of Chemistry, before being promoted to Reader (2017) and then Professor (2019) at the same institution. Vijay's research has been highlighted by Forbes, Scientific American, CNN News, Nature Chemistry and the Royal Society of Chemistry. protect (and subsequently deprotect) the thiol side chain to overcome these challenges, and to further expand upon the synthesis of biomolecules. Indeed, protection of Cys during standard peptide synthesis is therefore often critical to avoid undesired alkylation/oxidation, allowing for synthesis of the desired peptide. In the case of disulfide-containing peptide synthesis, the development of "orthogonal" Cys protecting groups (which can be selectively deprotected in the presence of one another) has proved paramount to ensuring regioselective disulfide formation. ${ }^{6}$

Herein we review the different thiol protecting groups reported for Cys throughout the last $70+$ years. We analyse and discuss each of the individual $60+$ protecting groups that have been reported for Cys protection (and deprotection). For each group (and where applicable), this review will focus on (a) the conditions used for its deprotection, (b) use in Boc and Fmoc peptide synthesis, (c) its comparison to other related Cys protecting groups, and (d) notable applications of the Cys protecting group in peptide and protein chemistry.

\section{Organisation of review}

This review begins with a brief introduction to key concepts relevant to the content covered within the review and Cys protecting group chemistry as a whole. This is then followed by a table (Table 1) and a series of discussions about each individual protecting group. Taking inspiration from previously reported literature, ${ }^{4-6}$ the protecting groups within this review have been divided into separate categories based on the conditions that have primarily been used for their deprotection. We note that, in cases where multiple methods of deprotection are available, certain protecting groups do not sit rigidly within these categories. The categories are as follows: Acid labile, oxidatively labile, base labile, enzymelabile, hydrazine-labile, palladium-labile, and reductively labile. The "benzyl" protecting group, protecting groups specific to $\mathrm{N}$-terminal Cys, and the "safety catch" protecting group, have also been assigned to their own separate categories. Additionally, in order to give historical context, the groups in each category are broadly arranged in chronological order. For additional reading, we direct the reader to extensive reviews on general amino acid protecting groups by Isidrio-Llobet, Álveraz, and Albericio, ${ }^{4}$ along with reviews that focus on regioselective disulfide formation by Postma, and Albericio, ${ }^{6}$ and more recently by He, Pan, Mayer, and Li. $^{5}$ We also wish to highlight a recent review by Laps, Satish, and Brik discussing recent advances in transition metal chemistry relating to peptide/protein synthesis. ${ }^{25}$

\section{Key concepts in cysteine protecting group chemistry}

\subsection{Solid phase peptide synthesis}

Briefly, chemical peptide synthesis dates back well over 100 years ago, when Curtius synthesised benzoylglycylglycine, an N-protected dipeptide, in 1882 using benzoyl chloride and the silver salt of glycine. ${ }^{17}$ In the early 1900 s, Fischer and Fourneau reported the 


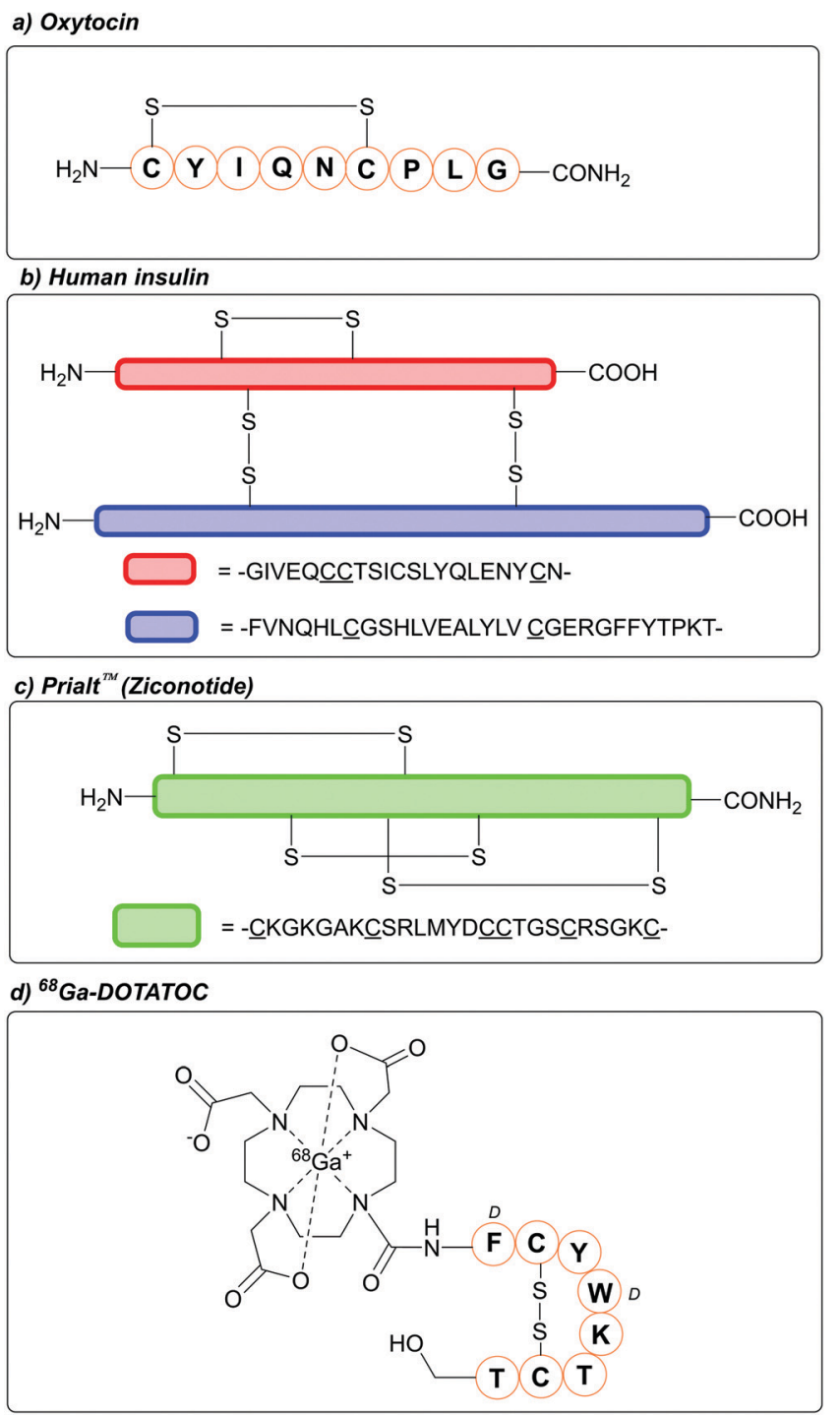

e) Adcetris $^{\text {TI }}$ (Brentuximab vedotin)

.

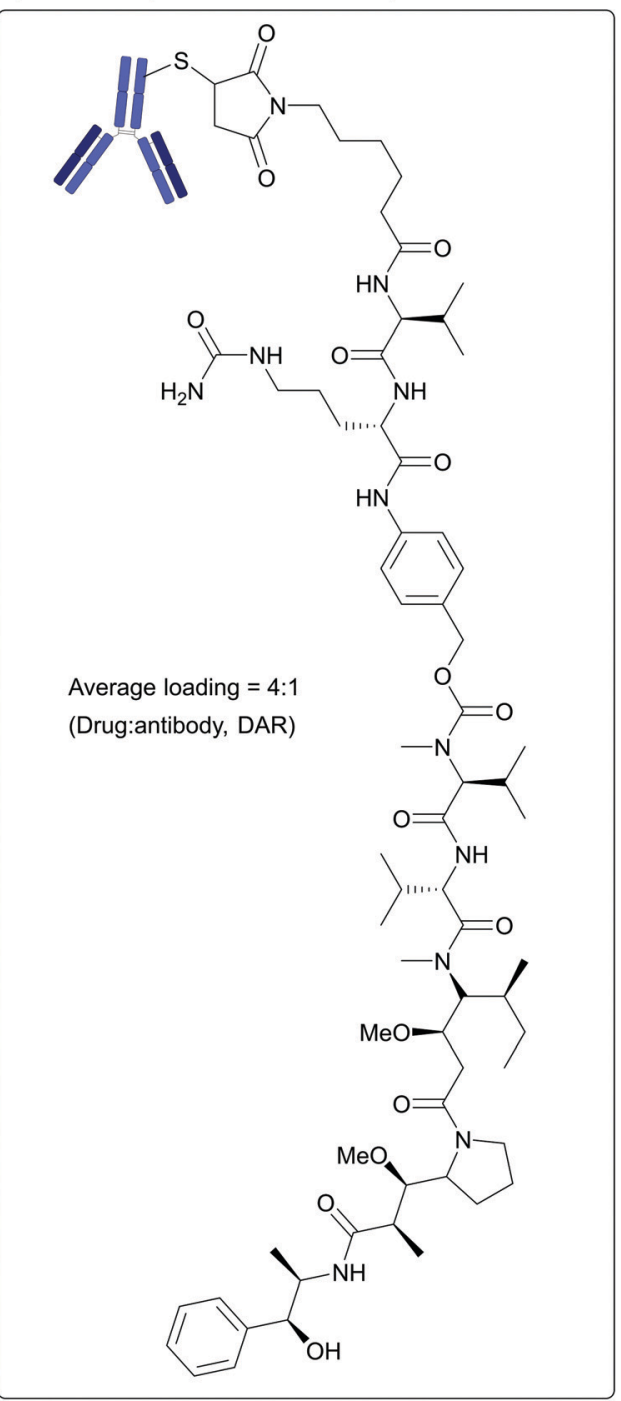

Fig. 1 Examples of Cys-containing biomolecules of therapeutic interest. (a) Oxytocin, (b) Human insulin, (c) Prialt ${ }^{\mathrm{TM}} /$ Ziconotide, (d) ${ }^{68}$ Ga-DOTATOC, (e) Adcetris ${ }^{\mathrm{TM}} /$ Brentuximab vedotin.

synthesis of the unprotected dipeptide via hydrolysis of glycine ester to give the diketopiperazine, which subsequently hydrolysed to give glycylglycine. ${ }^{26}$ Later, in 1932, the concept of using protecting groups to temporarily mask functional groups in the context of peptide synthesis was established when Bergmann and Zervas introduced the carbobenzoxy group ( $\mathrm{Cbz}$ or $\mathrm{Z}$ ) for the protection of amines. ${ }^{26}$ Another key concept was then introduced in 1955 by Sheehan and Hess, where $N, N$-dicyclohexylcarbodiimide (DCC) was established as an amide coupling reagent for peptide synthesis. ${ }^{27}$ This would later be followed by the introduction of the acid-labile tert-butyloxycarbonyl (Boc) protecting group in 1957 by Carpino, ${ }^{28}$ Mckay, and Albertson. ${ }^{29}$ Peptide synthesis would later be revolutionised by the advent of solid phase peptide synthesis (SPPS) reported in 1963 by Merrifield. ${ }^{30}$ Here, peptides are synthesised through sequential coupling of protected amino acids on a solid support (typically a polystyrene resin); impurities and excess reagents can then be removed by simple wash and filtration of the solid support (Fig. 2). Specific cocktails of reagents can then be used to cleave and isolate the peptide from the resin; different resins have since been reported over the last few decades, which yield peptides with different functional groups at the $C$-terminus once cleaved e.g. acid, amide, thioester. ${ }^{31}$ Carpino, along with Han, would then introduce the base-labile 9-fluorenylmethoxycarbonyl (Fmoc) protecting group in $1970 .^{32}$ The combination of these concepts has led to development of two solid phase strategies for peptide synthesis: the Boc/Benzyl (Bn) strategy, and the Fmoc/tert-butyl ( $t \mathrm{Bu})$ strategy. The Fmoc/ $t \mathrm{Bu}$ strategy is now the more routinely used, as it avoids both the repeated acidic treatments of the peptide, and the use of hydrogen fluoride (HF) (which requires specialist equipment and is extremely toxic) that is required in Boc/Bn peptide synthesis. ${ }^{33}$ For a more extensive breakdown of the history of peptide chemistry and related key concepts, we direct the reader's attention to discussions in recently published reviews. ${ }^{33,34}$ 
Solid phase peptide synthesis (SPPS)

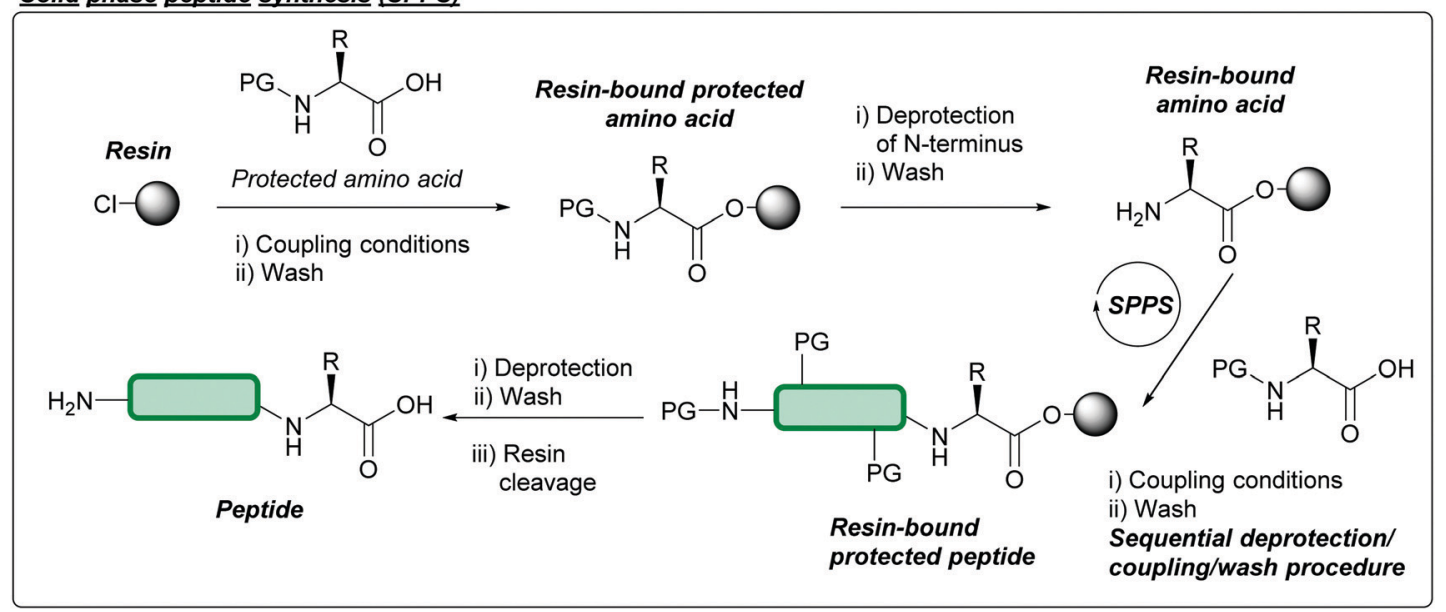

Fig. 2 Outline of solid phase peptide synthesis (SPPS) procedure. Following loading of a desired amino acid onto a functionalised polystyrene resin, sequential deprotections of the $\mathrm{N}$-terminal protecting group and amide coupling reactions yield a resin-bound, protected peptide of a desired sequence. Excess reagents/impurities can be removed by washing and filtration at each step. Removal of protecting groups and cleavage of the peptide off the resin with specific cocktails of reagents yields the final desired peptide.

\subsection{Native chemical ligation and related ligations strategies}

SPPS is now the standard for the chemical peptide synthesis; however, the process is generally limited to peptides of $c a .50$ amino acids long. ${ }^{35}$ As such, ligation reactions are usually employed when synthesising larger peptides, ${ }^{36}$ the most common of which is the native chemical ligation (NCL) strategy. Introduced in 1994 by Dawson, Muir, Clark-Lewis, and Kent, ${ }^{37}$ NCL is a ligation reaction between a C-terminal peptide thioester and an $\mathrm{N}$-terminal cysteinyl peptide. An initial exchange reaction occurs between the thiol and the thioester linking the two peptides via a thioester bond. The molecule then spontaneously rearranges via an intramolecular $S, N$-acyl transfer, resulting in the formation of a native peptide bond (Fig. 3a). ${ }^{38}$ A notable example of NCL is in the one-pot synthesis of Crambin reported in 2004, whereby three peptide fragments were ligated together using NCL, followed by folding to yield the desired protein. ${ }^{39}$ Peptide fragments for NCL can be synthesised chemically via SPPS or, when significantly longer peptide fragments are desired, can be produced recombinantly in bacterial expression systems such as in Escherichia coli (E. coli) cells. In some cases, these recombinantly produced peptides contain an intein segment fused to the C-terminus, which can then undergo $N, S$-acyl shift and, following addition of an exogenous thiol such as sodium 2-mercaptoethanesulfonate (MESNa), cleavage to yield a thioester-containing peptide fragment that can subsequently participate in NCL; this process is known as expressed protein ligation (EPL, Fig. 3b). ${ }^{40,41}$ The resulting Cys residue post-NCL can then be converted to alanine through metal-based desulfurisation, ${ }^{42}$ or metal-free-based desulfurisation using radical initiators such as 2,2'-azobis[2-(2-imidazolin2-yl)propane]dihydrochloride (VA-044) in combination with tris(2-carboxyethyl)phosphine (TCEP, Fig. 3c). ${ }^{43}$ As a result, it is therefore common for alanine within a target peptide sequence to be the ligation site of choice.

Other common systems used for ligation of large peptides are $N, S$-acyl shift systems, one example of which is the cysteinylprolyl ester (CPE) system. ${ }^{44}$ Here, peptides containing a CPE unit at the C-terminus can undergo spontaneous $\mathrm{N}->S$ transformations into diketopiperazine thioesters. The resulting thioester can then undergo NCL in a one-pot process (Fig. 3d). A related system that uses cysteinylprolyl imide (CPI) peptides has more recently been described and used in the synthesis of proteins such as ZHER2 affibody. ${ }^{45}$ In addition, peptides containing C-terminal hydrazides can be used as more chemically stable peptide thioester precursors. ${ }^{46}$ In this strategy the hydrazide group can be chemically converted to a C-terminal azide or acyl pyrazole using nitrous acid or acetyl acetone, followed by conversion to a thioester through addition of 4-mercaptophenylacetic acid (MPAA, Fig. 3(e)). ${ }^{47}$ This methodology has since been used in the synthesis of multiple proteins, including Centruroides suffusus suffusus toxin II protein (CssII) ${ }^{46}$ and $\alpha$-synuclein. ${ }^{48}$ Peptide hydrazides can also be prepared through Cys cyanylation using 2-nitro-5-thiocyanatobenzoic acid (NTCB) followed by addition of hydrazine, as shown in the recently described activated Cys-directed protein ligation (APCL). ${ }^{49}$ Another ligation strategy is the thioester method, whereby a silver $\left(\mathrm{Ag}^{+}\right)$activated C-terminal alkyl thioester is directly displaced by an $\mathrm{N}$-terminal amine to yield the desired amide bond. ${ }^{50}$ A silver-free protocol has also been developed for the thioester method, which involves the use of aryl, as opposed to alkyl, thioesters (Fig. 3f). ${ }^{51}$ To ensure a successful peptide synthesis both thioester methods require peptide fragments with protected amino groups, such as Fmoc protection of $\mathrm{N}$-terminal $\alpha$-amino groups, or Boc/Z protection of Lys side chain $\varepsilon$-amino groups; alternatively, Lys side chains can be protected by using an azido-lysine analogue during peptide synthesis, followed by azide reduction to give the desired $\varepsilon$-amino group post-thioester ligation. ${ }^{52}$ Thioester methods have since been utilised for the synthesis of proteins such as chemokine CCL $27^{51}$ and various glycoproteins. ${ }^{53}$ For further reading on ligation methods, we direct the reader to extensive reviews on the subject. ${ }^{53-57}$ 
a) Native chemical ligation (NCL)

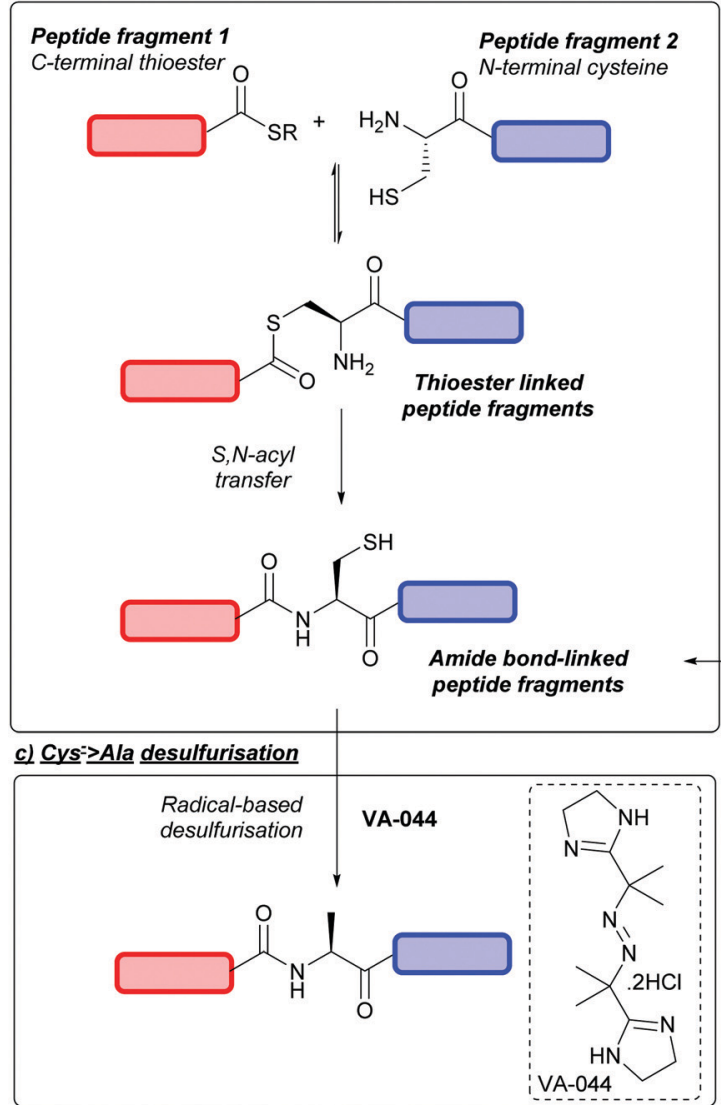

e) Peptide hydrazides

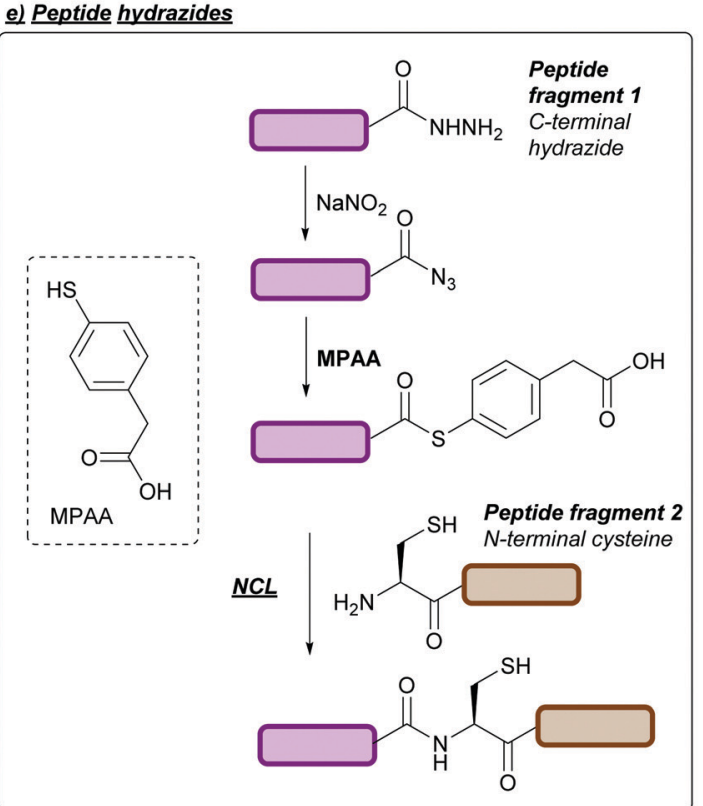

b) Expressed protein ligation EPL)

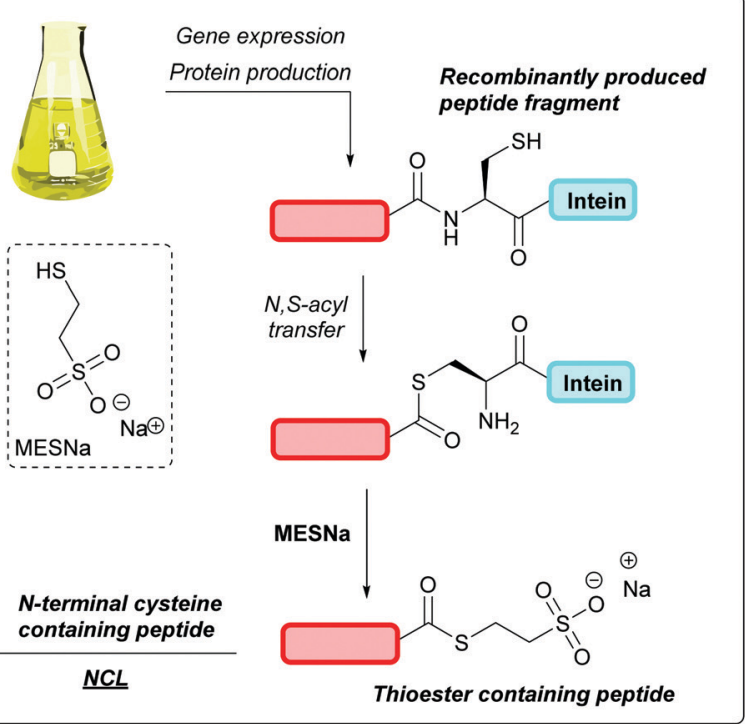

\section{d) Cysteinyl prolyl ester (CPE) system}
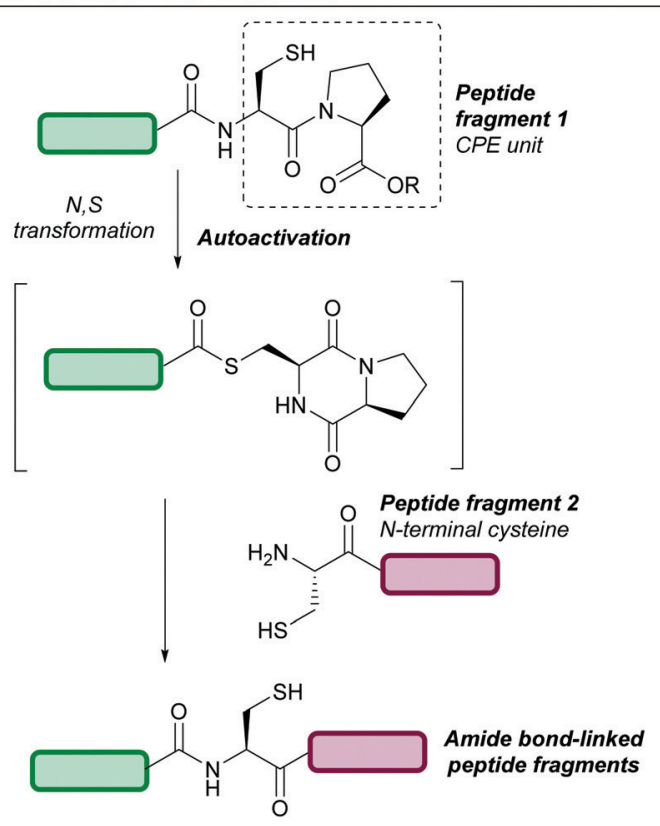

f) Thioester method

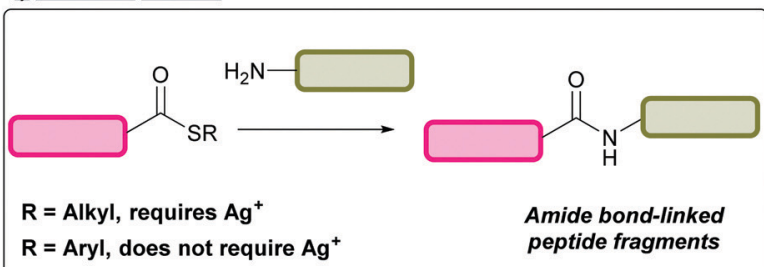

Fig. 3 Strategies related to peptide ligation. (a) Native chemical ligation (NCL), (b) expressed protein ligation (EPL), (c) radical-based desulfurisation of Cys to yield alanine using 2,2'-azobis[2-(2-imidazolin-2-yl)propane]dihydrochloride (VA-044), (d) cysteinyl prolyl ester(CPE) system, (e) ligation of peptides using peptide hydrazides, (f) thioester method for ligation of peptides.

\subsection{Orthogonality and bioorthogonality}

In 1977, Barany and Merrifield described the concept of "orthogonality", which (as mentioned previously) describes protecting groups that can be chemoselectively removed in the presence of one another without affecting/removing each other under certain sets of conditions. ${ }^{58}$ This concept has 
proved especially important in peptide synthesis when considering disulfide-containing peptides, where the use of protecting groups can avoid off-target and undesired disulfide formation (Fig. 4a). ${ }^{5,6}$ The concept of orthogonality would later be extended to chemical biology when Bertozzi coined the term "bioorthogonal". First referenced by Bertozzi in 2003, along with Hang, Yu, and Kato, ${ }^{59}$ bioorthogonal/bioorthogonality refers to chemical reactions that can be performed in biological systems without interfering with the native environment (Fig. 4b). ${ }^{60,61}$ This typically relies on incorporation of non-natural functionalities, which can be used as "handles" for selective, biooorthogonal reactions. The field of bioorthogonal chemistry has grown rapidly over the last two decades, with in vivo applications such as metabolic labelling of developing zebrafish embryos. ${ }^{62,63}$ On the related subject of protein modification, advances in synthetic biology and genetic code expansion have enabled the incorporation of non-natural functionalities into proteins via unnatural amino acid mutagenesis. This typically involves expression of a gene (encoding for a protein of interest) containing an amber STOP codon (in E. coli cells), along with expression of an orthogonal tRNA/tRNA synthetase in the presence of an unnatural amino acid, to produce the unnatural amino acid-containing protein of interest (Fig. 4c).

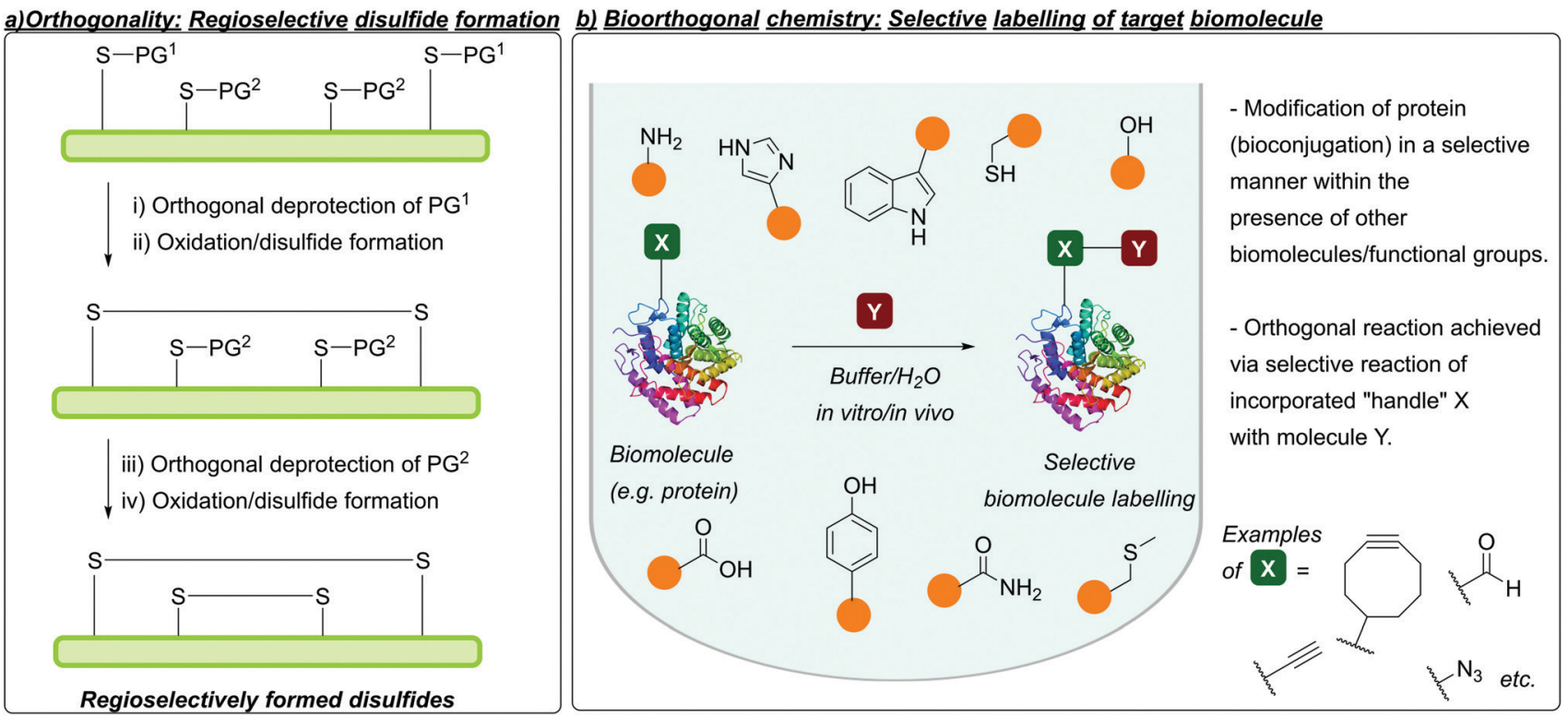

c) Unnatural amino acid mutagenesis: Installing protected amino acid residues

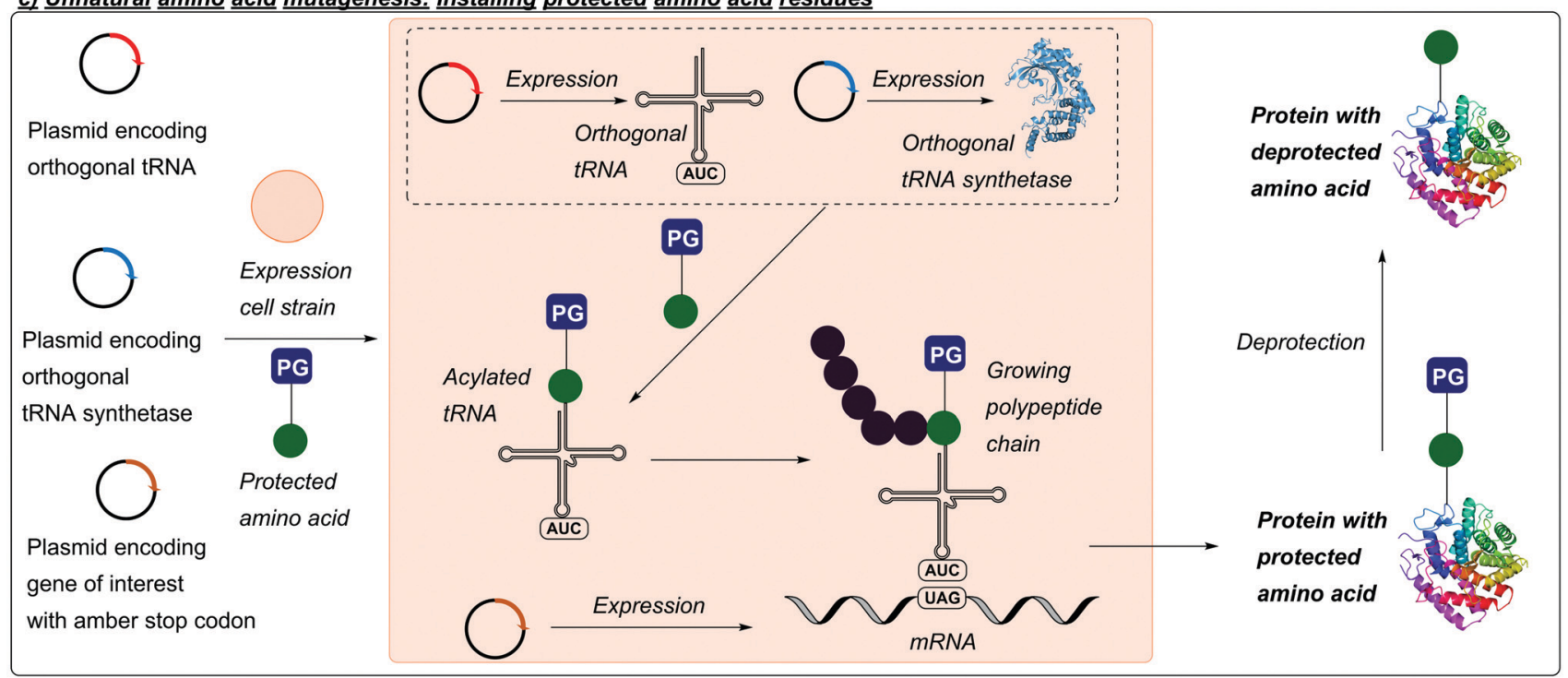

Fig. 4 (a) Concept of orthogonality and its relation to protecting group chemistry and disulfide formation. Using combinations of protecting groups that are orthogonal i.e. can be removed in the presence of one another, followed by disulfide bond formation in a sequential manner allows for controlled, regioselective disulfide bond formation. (b) Outline of bioorthogonal chemistry. Selective modification of a given biomolecule is carried out without off-target modification/perturbation of the native environment. (c) Outline of unnatural amino acid mutagenesis. Using a suitable cell strain, expression of a plasmid encoding for the desired protein (which contains an amber STOP codon) alongside an orthogonal tRNA synthetase/tRNA pair and unnatural amino acid allows for incorporation of said unnatural amino acid at the aforementioned STOP position. 
Functionalities introduced into proteins in this manner include azides, alkynes, aldehydes, and tetrazines. Similar to the thiol side chain of Cys, these groups can subsequently be used as handles for site-selective bioconjugation to yield conjugates such as ADCs. ${ }^{64}$ Alternatively, amino acids with modified or protected side chains can also be introduced; these include analogues that mimic post-translational modifications (PTMs) in proteins e.g. histones, ${ }^{65}$ or photocaged analogues of inactive enzymes which can subsequently be activated via deprotection e.g. photocaged Tyr in $\beta$-galactosidase. ${ }^{66}$

\section{Table of cysteine protecting groups}

\section{Cysteine protecting groups in peptide and protein science}

\subsection{Benzyl (Bzl)}

The benzyl (Bzl, Bn) protecting group (Fig. 5a) has been used for many years. ${ }^{67,167}$ One of the most noteworthy examples where the Cys(Bzl) protecting group has featured is in the landmark chemical synthesis of the hormone oxytocin in 1953 (Fig. 5b); this was the first example of a polypeptide hormone synthesis, and contributed towards Vincent du Vigneaud being awarded the Nobel Prize in Chemistry in 1955. Synthesis of other Cys-containing oxytocin analogues have also employed the Bzl protecting group. ${ }^{168}$ Additionally, Cys(Bzl) protected tripeptides have been shown to display apoptotic activity. ${ }^{169}$ Protocols for

Table 1 List of protecting groups for the Cys thiol that have been reported over the last several decades. The following for each protecting group is covered in this review: (i) name and abbreviations, (ii) the deprotection/lability classification (as mentioned previously in Section 2) (iii) deprotection conditions and relevant references (iv) conditions to which the protecting group is stable to/compatible with, and (v) the structure of Cys-protecting group conjugate as observed within a peptide chain

Protecting group, abbreviations and location within review Lability Deprotection conditions Stable to/compatible with Structure

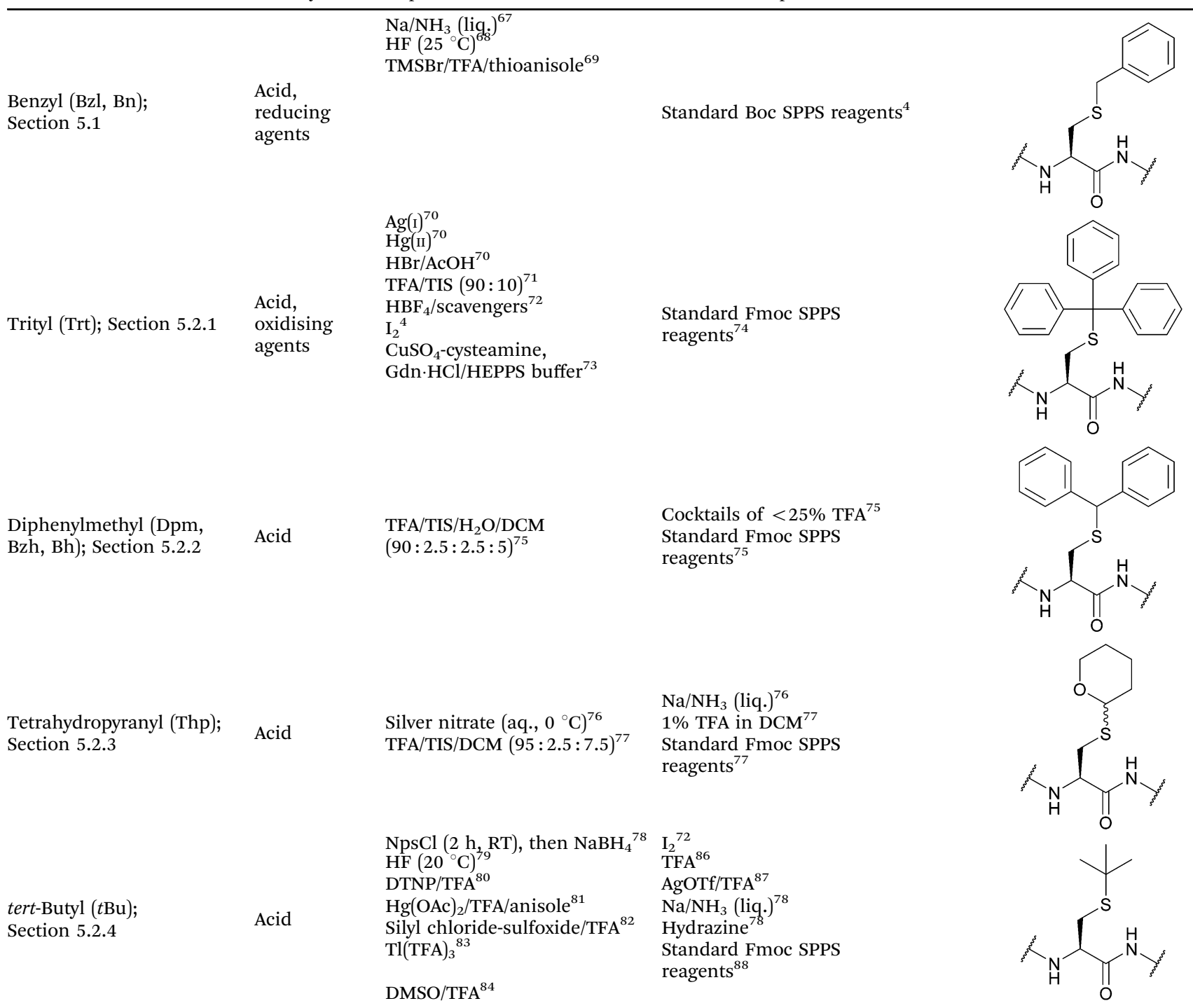


Table 1 (continued)

Protecting group,

abbreviations and

location within review

4-Methoxybenzyl (Mob, MBzl); Section 5.2.5

Acid

Lability

Deprotection conditions

Stable to/compatible with

Structure

$\mathrm{PdCl}_{2}, 50 \mathrm{mM}$ Tris or urea

buffer $\left(37^{\circ} \mathrm{C}\right)^{85}$

TFA $\left(100{ }^{\circ} \mathrm{C}\right)^{89}$

$\mathrm{HF}^{79}$

DTNP or DTP/TFA/

thioanisole ${ }^{90}$

$$
\text { MBzl); Section 5.2.5 }
$$$$
\text { TFA/TIS }\left(12 \mathrm{~h}, 37^{\circ} \mathrm{C}\right)^{86}
$$$$
\mathrm{Hg}(\mathrm{TFA})_{2}{ }^{81}
$$$$
\mathrm{Tl}(\mathrm{TFA})_{3}{ }^{83}
$$$$
\text { AgOTf/TFA/thioanisole }{ }^{87}
$$

$$
\mathrm{HBr}^{89}
$$

TFA (without scavengers) ${ }^{86}$

Standard Fmoc SPPS

reagents ${ }^{75}$

Standard Boc SPPS reagents

(for small peptides) $^{4,91}$

$50 \%$ TFA in DCM $(23 \mathrm{~h}$,

$\left.24{ }^{\circ} \mathrm{C}\right)^{92}$

Standard Boc SPPS reagents ${ }^{92}$

3,4-Dimethylbenzyl (DMB); Section 5.2.6

Acid

Methylbenzyl (Meb, 4-MeBn, 4-MeBzl);

Section 5.2.7

Acid

$$
\text { HF/anisole }\left(1 \mathrm{~h}, 0{ }^{\circ} \mathrm{C}\right)^{91}
$$

$\mathrm{HF}\left(10 \mathrm{~min}, 0{ }^{\circ} \mathrm{C}\right)^{92}$

1-Adamantyl (Ad, 1-Ada); Section 5.2.8

Acid

Benzyloxymethyl (Bom); Section 5.2.9

Acid

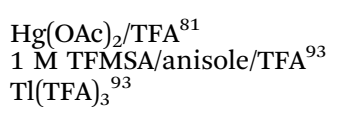

TFA $\left(2.5 \mathrm{~h}, 0{ }^{\circ} \mathrm{C}\right)^{93}$ AgOTf/TFA

Standard Boc SPPS reagents ${ }^{87}$ Standard Fmoc SPPS reagents ${ }^{4}$

$\mathrm{AgOTf}^{87}$

Standard Fmoc SPPS reagents ${ }^{74}$

DMSO/TFA $\left(45^{\circ} \mathrm{C}\right)^{74}$

Standard Boc SPPS reagents ${ }^{91}$

AgOTf/anisole/TFA $\left(1 \mathrm{~h}, 0{ }^{\circ} \mathrm{C}\right)^{94}$ TFA $\left(4 \mathrm{~h}, 0{ }^{\circ} \mathrm{C}\right)^{94}$

$1 \mathrm{M}$ TMSOTf/thioanisole/TFA Hydrazine ${ }^{94}$

$\left(1 \mathrm{~h}, 0{ }^{\circ} \mathrm{C}\right)^{94}$

Tl(TFA $)_{3}{ }^{94}$

Piperidine $/ \mathrm{DMF}^{94}$

$\mathrm{NaBO}_{3}{ }^{94}$

Standard Fmoc SPPS reagents $^{94}$

$\geq 6 \%$ TFA with TES or TIS

$(0.5 \%)$ in DCM $\left(5 \min , 25^{\circ} \mathrm{C}\right)^{95}$ $\geq 30 \%$ TFA in DCM with phenol/thioanisole $/ \mathrm{H}_{2} \mathrm{O}$

2,4,6-Trimethoxybenzyl (Tmob); Section 5.2.10

Acid

$$
(5 \% \text { each })^{95}
$$$$
\mathrm{I}_{2} / \mathrm{DMF}\left(0{ }^{\circ} \mathrm{C}\right)^{95}
$$

Standard Fmoc SPPS $\mathrm{Tl}(\mathrm{TFA})_{3} / \mathrm{DMF} /$ anisole $\left(0^{\circ} \mathrm{C}\right)^{95}$ reagents ${ }^{96}$<smiles>COc1ccc(CSC[C@H](NC(C)C)C(=O)NC(C)C)cc1</smiles><smiles>Cc1ccc(CSC[C@H](NC(C)C)C(=O)NC(C)C)cc1C</smiles><smiles>CSCc1ccc(C)cc1</smiles><smiles>CC(C)NC(=O)[C@H](CS)NC(C)C</smiles><smiles>CC(C)NC(=O)[C@H](CSC12CC3CC(CC(C3)C1)C2)NC(C)C</smiles><smiles>CC(C)NC(=O)[C@H](CSCOCc1ccccc1)NC(C)C</smiles>

$\mathrm{MeO}$

Standard Fmoc SPPS

$4,4^{\prime}, 4^{\prime \prime}$,-Trimethoxytriphenylmethyl (TMTr); $\quad$ Acid $1 \%$ TFA in $\mathrm{DCM}^{96}$ reagents $^{96}$ 
Table 1 (continued)

Protecting group,

abbreviations and

location within review

Pseudoprolines ( $\Psi$ Pro);
Section 5.2.12

Section 5.2.12

Acid

Lability

Deprotection conditions

Stable to/compatible with

Structure

TFA ( $\Psi^{\mathrm{Me}, \mathrm{Me}}$ pro, 32-36 h, Standard Fmoc SPPS

sequence dependant ${ }^{97}$

TFMSA $\left(15 \mathrm{~min}, 0{ }^{\circ} \mathrm{C}\right)^{98}$

TFA ( $\Psi^{\mathrm{Me}, \mathrm{Me}}$ pro, $1-6 \mathrm{~h}$,

reagents ${ }^{97}$

TFA $\left(\Psi^{\mathrm{H}, \mathrm{H}} \text { pro }\right)^{97}$

TFA/MeOH/TIS/ $\mathrm{H}_{2} \mathrm{O}$

$\left(80: 15: 2.5: 2.5, \Psi^{\mathrm{Me}, \mathrm{Me}}\right.$ pro, 1

h, cyclic peptides) ${ }^{101}$

TFA ( $\Psi^{\mathrm{H}, \mathrm{Dmp}}$ pro, minutes $)^{97}$

TFA ( $\Psi^{\mathrm{H}, \mathrm{Dmp}}$ pro, C-terminal

resin-bound, $1.5 \mathrm{~h})^{100}$

4-Methyltrityl (Mtt);

Section 5.2.13

Acid

$1 \%$ TFA/scavengers ${ }^{75}$

1-3\% TFA in DCM/TES

4-Methoxytrityl (Mmt);

Section 5.2.13

Acid

9H-Xanthen-9-yl (Xan);

Section 5.2.15

Acid

2-Methoxy-9H-xanthen-9-yl (2-Moxan); Section 5.2.15

Acid

4,5,6-Trimethoxy-2,2-

dimethyl-2,3-dihydrobenzo- Acid furan-7-methyl (Tmbm);

Section 5.2.16

2,2,5,7,8-Pentamethylchroman-6-methyl

(Pmcm); Section 5.2.16
Acid
$\left.\mathrm{I}_{2}{ }^{71}: 5\right)^{71}$

TFA: DCM : TES

$(0.1: 99.4: 0.5)^{102}$

TFA: DCM : BME $(10: 85: 5)^{102}$

TFA: DCM: TES $(1: 98.5: 0.5)$

$\mathrm{I}_{2} / \mathrm{MeOH}^{102}$

$\mathrm{Tl}(\mathrm{TFA})_{3}{ }^{102}$

TFA: DCM : TES

$(0.1: 99.4: 0.5)^{102}$

TFA: DCM: BME $(10: 85: 5)^{102}$

TFA: DCM: TES $(1: 98.5: 0.5)$

solid phase $(25 \mathrm{C}, 2 \mathrm{~h})^{102}$

$\mathrm{I}_{2} / \mathrm{MeOH}^{102}$

$\mathrm{Tl}(\mathrm{TFA})_{3}{ }^{102}$

iperidine/DMF ( $\geq 24 \mathrm{~h}$, $\left.25{ }^{\circ} \mathrm{C}\right)^{102}$

HOBt/DMF $\left(24 \mathrm{~h}, 25^{\circ} \mathrm{C}\right)$;

$\mathrm{AcOH}^{102}$

Very weak acids, e.g. AcOH/ TFE/DCM $(1: 2: 7,30 \mathrm{~min})^{71}$ Standard Fmoc SPPS reagents $^{71}$

Standard Fmoc SPPS reagents ${ }^{102}$

Piperidine/DMF $(\geq 24 \mathrm{~h}$, $\left.25{ }^{\circ} \mathrm{C}\right)^{102}$

HOBt/DMF $\left(24 \mathrm{~h}, 25^{\circ} \mathrm{C}\right)$;

$\mathrm{AcOH}^{102}$

Standard Fmoc SPPS reagents ${ }^{102}$

TFA/TES/DCM $(1: 5: 94)^{103}$

Standard Fmoc SPPS reagents ${ }^{103}$

Standard Fmoc SPPS reagents ${ }^{103}$
DMF $\left(24 \mathrm{~h}, 22{ }^{\circ} \mathrm{C}\right)^{71}$<smiles>C/C=C(/CSC)C(=COC)OC</smiles><smiles>CCNC(CSCC)C(=O)NC(C)C</smiles><smiles>Cc1c(C)c2c(c(C)c1CS)OC(C)(C)CC2</smiles>

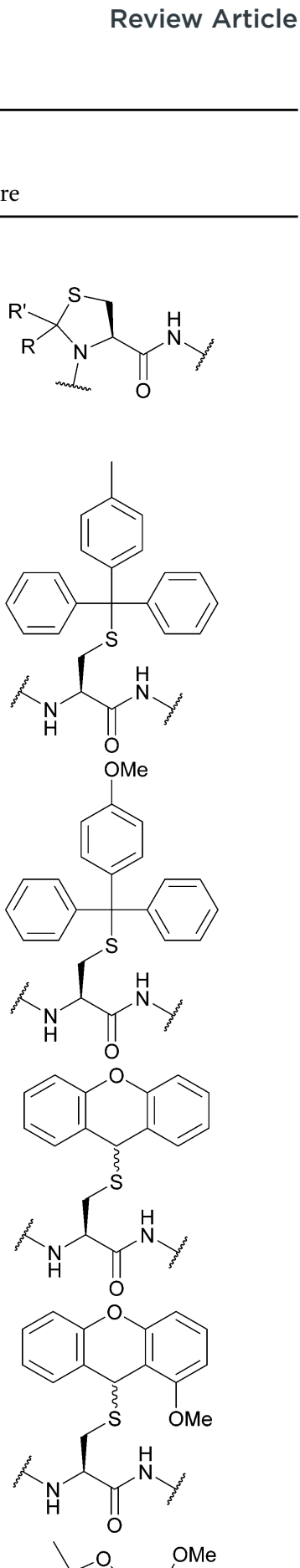

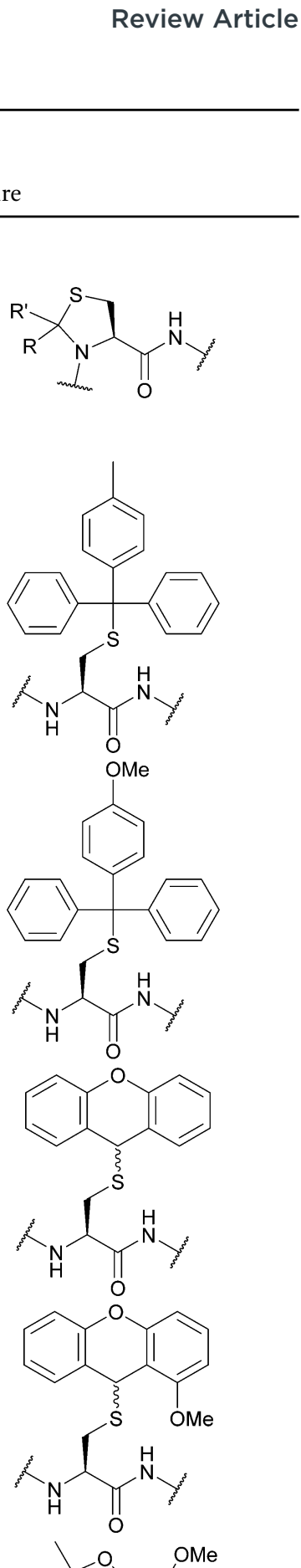

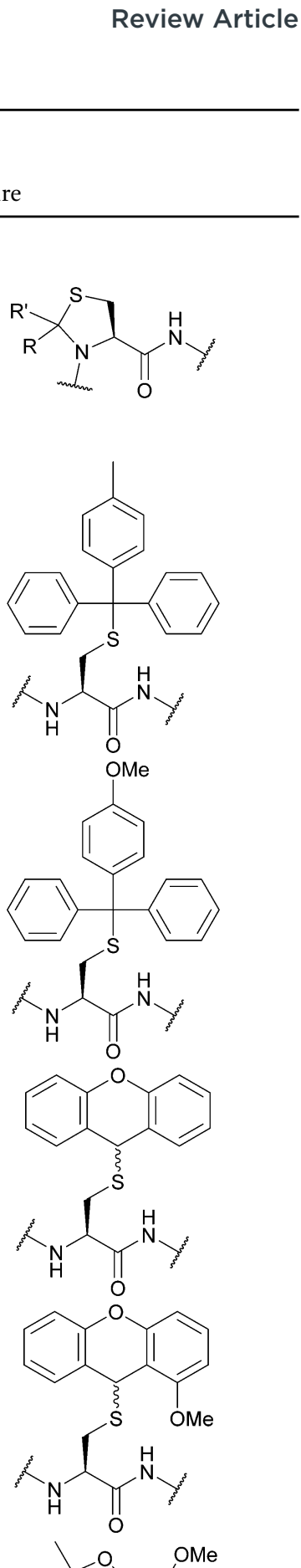

$\mathrm{OMe}$

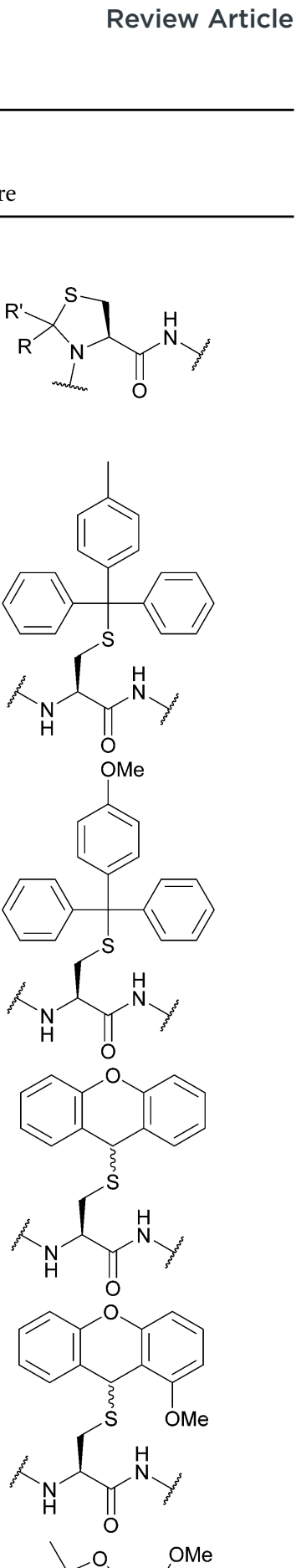

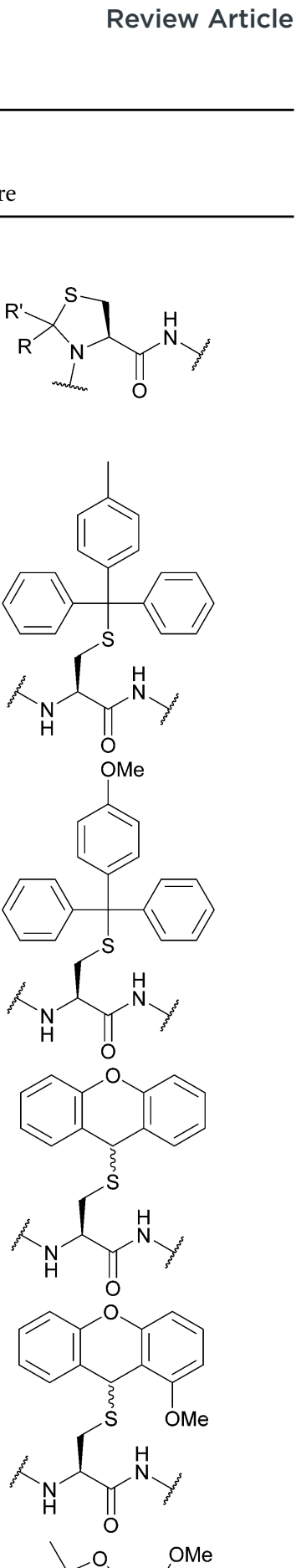

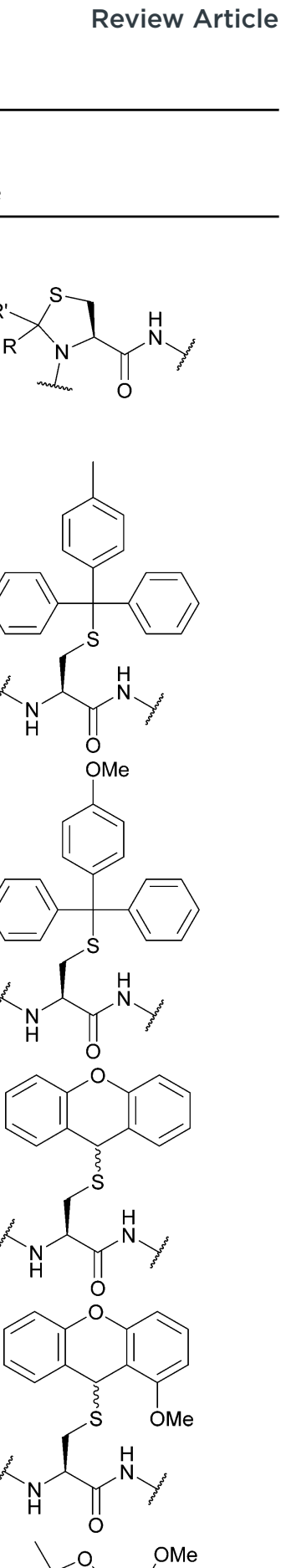

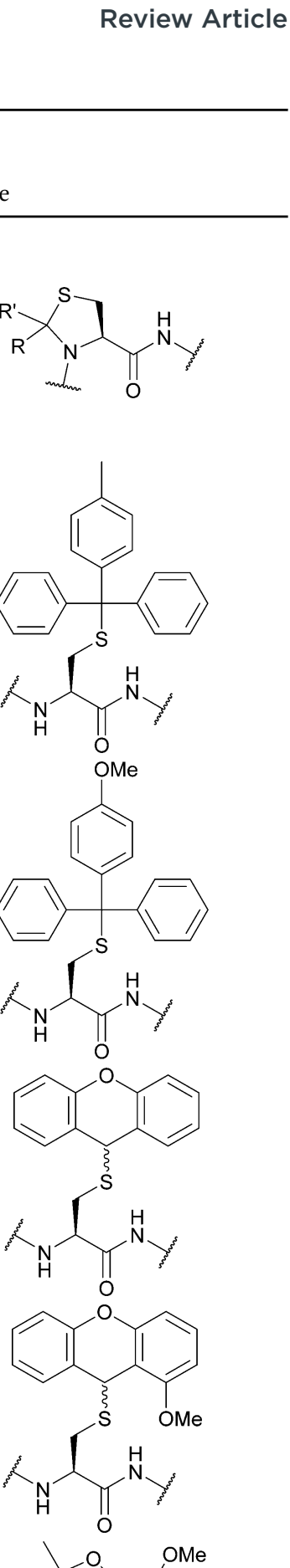

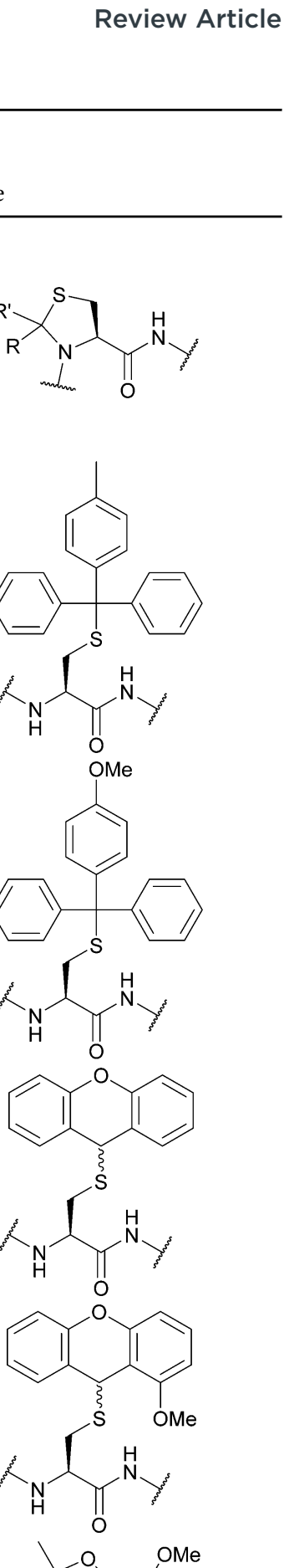

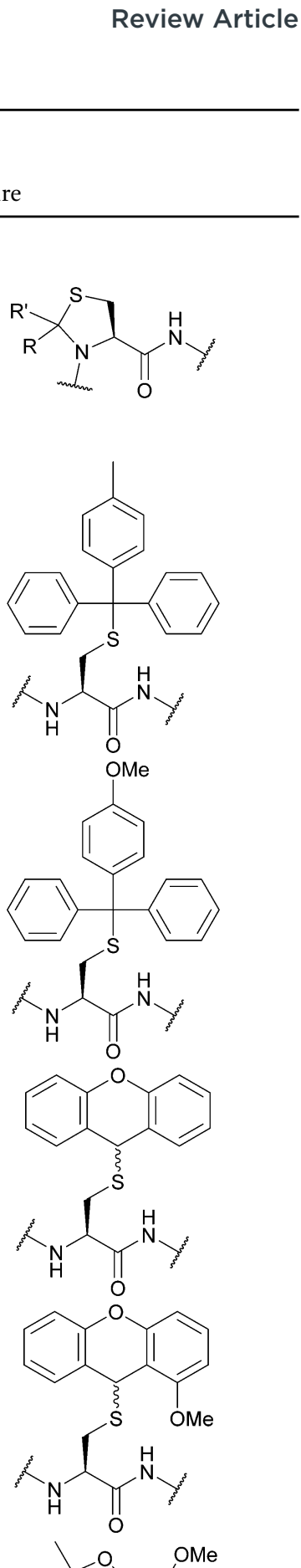<smiles>COc1ccc(C)cc1</smiles><smiles>CN[C@@H](CS)C(=O)NC(C)C</smiles> 
Table 1 (continued)

Protecting group,

abbreviations and location within review

Lability

Deprotection conditions

$\mathrm{T}_{2}{ }^{103} \mathrm{DCM} / \mathrm{TIS}(1: 5: 94)^{103}$

HFIP or TFE in DMF (15 min $)^{103}$

2,2,4,6,7-Pentamethyl-2,3dihydrobenzofuran-5-

methyl (Pbfm);

Acid

Section 5.2.16

4-Methoxybenzyloxymethyl (Mbom); Section 5.2.17

Acid

2,6-Dimethoxybenzyl (2,6diMeOBn); Section 5.2.18

Acid

Acid

(4-MeO-2MeBn);

Section 5.2.18

4,4'-Dimethoxydiphenyl-

methyl (Ddm); Section 5.2.19 Acid

$\mathrm{Hmb}^{\text {on/off }}$; Section 5.2.20 Acid

Acetamidomethyl (Acm); Section 5.3.1

TFA: DCM : TIS : $\mathrm{H}_{2} \mathrm{O}$

TFA : DCM : TIS : $\mathrm{H}_{2} \mathrm{O}$

$$
\begin{aligned}
& \text { Standard Fmoc SPPS } \\
& \text { reagents }^{103}
\end{aligned}
$$$$
\text { Reagent } \mathrm{K} / \mathrm{MeONH}_{2} \cdot \mathrm{HCl}^{104} \quad \begin{aligned}
& \text { Standard Fmoc SPPS } \\
& \text { reagents }^{104}
\end{aligned}
$$
$\left(50: 45: 2.5: 2.5,1 \mathrm{~h}, 25{ }^{\circ} \mathrm{C}\right)^{105}$ reagents $^{75}$

Standard Fmoc SPPS $\left(50: 45: 2.5: 2.5,1 \mathrm{~h}, 25{ }^{\circ} \mathrm{C}\right)^{105}$ reagents $^{75}$ $\begin{array}{ll}\text { TFA : DCM : TIS : } \mathrm{H}_{2} \mathrm{O} & \text { Standard Fmoc SPPS } \\ \left(10: 85: 2.5: 2.5,1 \mathrm{~h}, 25{ }^{\circ} \mathrm{C}\right)^{105} & \text { reagents }{ }^{106}\end{array}$ Standard Fmor
reagents

TFA/TIS/ $\mathrm{H}_{2} \mathrm{O}(95: 2.5: 2.5,2 \mathrm{~h}$, $25{ }^{\circ} \mathrm{C}$ ) in $\mathrm{Hmb}^{\text {off }}$ form ${ }^{107}$ Standard Fmoc SPPS reagents in $\mathrm{Hmb}^{\text {off }}$ form ${ }^{107}$

TFA/TIS/ $\mathrm{H}_{2} \mathrm{O}(95: 2.5: 2.5,2 \mathrm{~h}$, Standard NCL/desulfurisation/ $25{ }^{\circ} \mathrm{C}$ ) in $\mathrm{Hmb}^{\text {on }}$ form $^{107}$ HPLC reagents in $\mathrm{Hmb}^{\text {on }}$ form $^{107}$

$\mathrm{NpsCl}(2 \mathrm{~h}, \mathrm{RT})$, then $\mathrm{NaBH}_{4}{ }^{78}$
$\mathrm{Hg}(\mathrm{II})^{108} \mathrm{Ag}(\mathrm{I})^{87} \mathrm{Pd}(\mathrm{II})^{109}$
$6 \mathrm{M} \mathrm{HCl}\left(20 \mathrm{~h}, 110{ }^{\circ} \mathrm{C}\right)^{110}$
15 eq. DTNP in $97.5 \%$
TFA/thioanisole
98\%,90
$\mathrm{I}_{2}{ }^{111}$
Tl(TFA with scavengers ${ }_{3}{ }^{111}$

Silyl chloride-sulfoxide/TFA ${ }^{111}$
TFA $\left(25^{\circ} \mathrm{C}\right)^{110}$

$\mathrm{HBr} / \mathrm{AcOH}\left(25^{\circ} \mathrm{C}\right)^{110}$

$\mathrm{HCl} / \mathrm{EtOH}\left(25^{\circ} \mathrm{C}\right)^{110}$ $\mathrm{HF}\left(0^{\circ} \mathrm{C}\right)^{110}$

Oxidising agents
$\operatorname{Pd}(0)^{113}$

TCEP $^{114}$

Standard Fmoc SPPS reagents $^{74}$
Structure<smiles>CCNC(=O)C(CSCc1c(C)c(C)c2c(c1C)CC(C)(C)O2)NC(C)C</smiles><smiles>COc1ccc(COCSCC(NC(C)C)C(=O)NC(C)C)cc1</smiles><smiles>COc1cccc(OC)c1CSC</smiles><smiles>CCCC(NC(C)C)C(=O)NCC</smiles>

$\mathrm{MeO}$<smiles>CCc1ccc(C)cc1C</smiles><smiles>CCNC(=O)C(CSC)NC(C)C</smiles><smiles>COc1ccc(C(SC[C@H](NC(C)C)C(=O)NC(C)C)c2ccc(OC)cc2)cc1</smiles><smiles>CNCCN(C)C(=O)Oc1cc(OC)ccc1CSC[C@H](NC(C)C)C(=O)NC(C)C</smiles><smiles>CC(=O)NCSC[C@H](NC(C)=O)C(=O)NC(C)C</smiles> 
Table 1 (continued)

Protecting group,

abbreviations and

location within review
Lability

Deprotection conditions

Stable to/compatible with

Structure
$\mathrm{PdCl}_{2}$, aqueous/buffered

conditions ${ }^{112}$

$\mathrm{CuSO}_{4}$, aqueous/buffered

conditions with aminothiol

source $^{73}$

$\mathrm{Hg}(\mathrm{OAc})_{2}{ }^{115}$

$\mathrm{I}_{2} / \mathrm{MeOH}$ or $\mathrm{AcOH}^{115}$

$\mathrm{Tl}(\mathrm{TFA})_{3}{ }^{83}$

5-Dibenzosuberyl (Dbs, Sub); Section 5.3.2

Oxidising agents

Benzamidomethyl (Bam); Section 5.3.3

Oxidising agents

(2-Oxo-1-pyrrolidinyl)methyl (Pym); Section 5.3.4 agents

Dimethylphosphinothioyl (Mpt); Section 5.3.5

Oxidising agents

Trimethyl-acetamidomethyl (Tacm); Section 5.3.6

Oxidising

agents
$\mathrm{NH}_{3} / \mathrm{MeOH}^{122}$ $50 \%$ Piperidine in DMF $(2 \mathrm{~h}, \mathrm{RT})^{123}$

$\mathrm{Hg}(\mathrm{OAc})_{2}(1 \mathrm{~h}, \mathrm{RT})^{116}$ Ag(OTf)/TFA/anisole $\left(1 \mathrm{~h}, 0{ }^{\circ} \mathrm{C}\right)^{87} \quad 1 \mathrm{M} \mathrm{NaOH}\left(25^{\circ}{ }^{\circ} \mathrm{C}\right)^{11}$ Silyl chloride-sulfoxide/TFA ${ }^{82}$ TFA $\left(25^{\circ} \mathrm{C}\right)^{116}$ $6 \mathrm{M} \mathrm{HCl}\left(24 \mathrm{~h}, 110^{\circ} \mathrm{C}\right)^{116}$

$\mathrm{HF}^{117}$

$\mathrm{NaBO}_{3}{ }^{117}$

$\mathrm{AgNO}_{3}$ (2-4 equiv., $20 \mathrm{~min}, 1 \mathrm{~h}$ $\left.0{ }^{\circ} \mathrm{C}\right)$ in $\mathrm{H}_{2} \mathrm{O}^{118}$

in $\mathrm{H}_{2} \mathrm{O}^{118}$

TBAF in THF (free thiol) ${ }^{119} \quad 2 \mathrm{M} \mathrm{HCl} / \mathrm{AcOH}^{118}$

TBAF in DMF (disulfide) ${ }^{119} \quad 1 \mathrm{M} \mathrm{HCl} / \mathrm{H}_{2} \mathrm{O}^{118}$

TBAF in DCM

$\left(-\mathrm{S}-\mathrm{CH}_{2}-\mathrm{S}-\text { formation }\right)^{119}$

$\mathrm{Hg}(\mathrm{OAc})_{2}{ }^{120}$

$\mathrm{AgBF}_{4} / \mathrm{TFA} /$ thioanisole $^{121}$

$\mathrm{I}_{2} / \mathrm{EtOH}$ in $\mathrm{AcOH}^{120}$

Base
90\% $\mathrm{Zn} / \mathrm{AcOH}\left(0{ }^{\circ} \mathrm{C}\right)^{116}$

Standard Boc SPPS reagents ${ }^{116}$

Solution phase Boc reagents ${ }^{117}$

TFA $^{118}$

Solution phase Boc reagents ${ }^{118}$ $\mathrm{HF}\left(1 \mathrm{~h}, 0{ }^{\circ} \mathrm{C}\right)^{117}$

TFMSA/thioanisole/TFA

$\left(2 \mathrm{~h}, 0{ }^{\circ} \mathrm{C}\right)^{117}$

$0.05 \mathrm{M} \mathrm{NaOH}$ in $\mathrm{MeOH}$

(aq., $\left.1 \mathrm{~h}, 0{ }^{\circ} \mathrm{C}\right)^{117}$

Hydrazine/MeOH (24 h, RT) ${ }^{117}$

$\mathrm{Zn}$ in $90 \% \mathrm{AcOH}\left(1 \mathrm{~h}, 25^{\circ} \mathrm{C}\right)^{117}$

Standard Boc SPPS reagents ${ }^{93}$

$\mathrm{TFA}^{93}$

TFMSA/TFA ${ }^{93}$

$\mathrm{HCl}\left(110{ }^{\circ} \mathrm{C}\right)^{93}$

HF/anisole $\left(95: 5,1 \mathrm{~h}, 0{ }^{\circ} \mathrm{C}\right)^{93}$ $0.1 \mathrm{M} \mathrm{I}_{2} / \mathrm{DMF}^{12}$

$\mathrm{H}_{2}, \mathrm{Pd} / \mathrm{C}^{122}$

Standard Boc SPPS reagents ${ }^{123}$<smiles>CC(C)NC(=O)[C@H](CSC1c2ccccc2CCc2ccccc21)NC(C)C</smiles><smiles>CC(C)NC(=O)[C@H](CSCNC(=O)c1ccccc1)NC(C)C</smiles><smiles>CSCN1CCCC1=O</smiles><smiles>CC(C)NC(=O)[C@H](CS)NC(C)C</smiles><smiles>CC(C)NC(=O)[C@H](CSP(C)(C)=S)NC(C)C</smiles><smiles>CC(C)NC(=O)[C@H](CSCNC(=O)C(C)(C)C)NC(C)C</smiles><smiles>CC(C)N[C@@H](CSCC1c2ccccc2-c2ccccc21)C(=O)C(C)(C)C</smiles> 
Table 1 (continued)

Protecting group,

abbreviations and

location within review

Lability

Deprotection conditions

$50 \%$ Piperidine in DMF

$(30 \mathrm{~min})^{124}$

Dilute $\mathrm{DBU}^{68}$

2-(2,4-Dinitrophenyl)ethyl

(Dnpe); Section 5.4.2

Base

Hydroxyglycine-Acm

(Hgm); Section 5.6.1

9-Fluorenylmethyl-

oxycarbonyl (Fmoc);

Section 5.4.3

Base

$\mathrm{Et}_{3} \mathrm{~N}$, then $\mathrm{NH}_{3} / \mathrm{MeOH}$ or

$50 \%$ Piperidine in DMF

$(2 \mathrm{~h}, \mathrm{RT})^{125}$

Phenyl-acetamidomethyl (Phacm); Section 5.5.1

Hydroxyquinoline-Acm

(Hqm); Section 5.6.1

Allyloxycarbonyl (Alloc); Section 5.7.1
Penicillin $\mathrm{G}$ acylase

(pH 7.9 buffer) ${ }^{126}$

$\mathrm{Hg}_{127}(\mathrm{II})^{127} \mathrm{Ag}$ (II)

$\operatorname{Tl}(\mathrm{TFA})_{3}{ }^{127}$

Enzyme, oxidising agents

Hydrazine

$5 \%$ hydrazine in $\mathrm{H}_{2} \mathrm{O}$ $\left(\mathrm{pH} 8.5,3 \text { days, } 37^{\circ} \mathrm{C}\right)^{128}$

$5 \%$ hydrazine in $\mathrm{H}_{2} \mathrm{O}$ $\left(\mathrm{pH} 8.5,8 \mathrm{~h}, 37^{\circ} \mathrm{C}\right)^{128}$ $\mathrm{I}_{2}(30 \mathrm{~min})^{128}$

Hydrazine

$\mathrm{Pd}(0)$ cat. $/ \mathrm{Bu}_{3} \mathrm{SnH} / \mathrm{AcOH}^{126}$ Piperidine $\left(3 \mathrm{~h}, 30^{\circ} \mathrm{C}\right)^{129}$

Pd, base AgOAc $(30 \mathrm{~min})^{128}$
Stable to/compatible with Structure

5\% DIEA in DCM $(2 \mathrm{~h})^{124}$

$40 \%$ TFA in DCM $(24 \mathrm{~h})^{124}$ $90 \% \mathrm{HF} / p$-cresol or anisole

$\left(1 \mathrm{~h}, 0{ }^{\circ} \mathrm{C}\right)^{124}$

Tl(TFA) ${ }_{3} /$ TFA $^{124}$

$\mathrm{I}_{2}$ in $80 \% \mathrm{AcOH}$ (aq.) ${ }^{124}$

Standard Boc SPPS reagents ${ }^{124}$

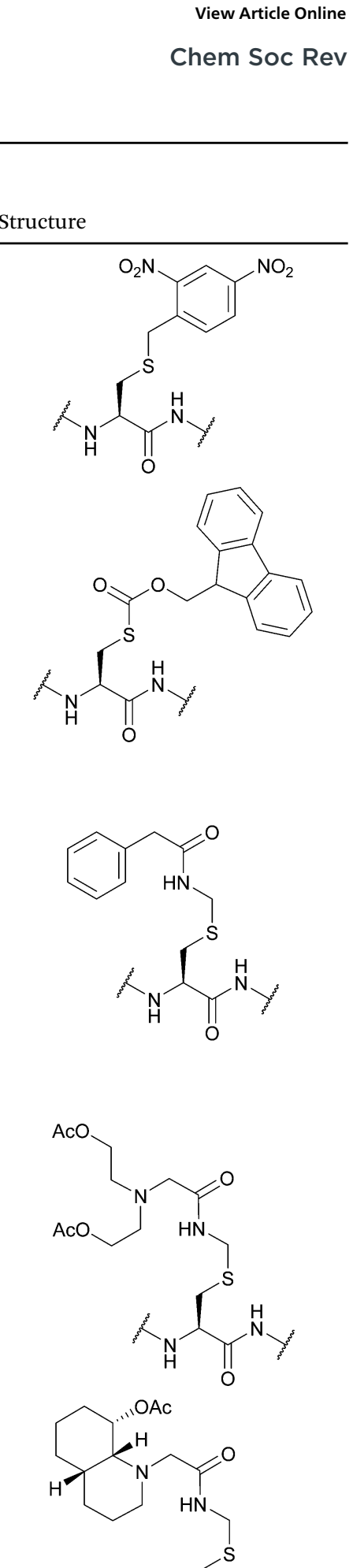

$4 \mathrm{M} \mathrm{HCl}$ in dioxane $\mathrm{e}^{125}$

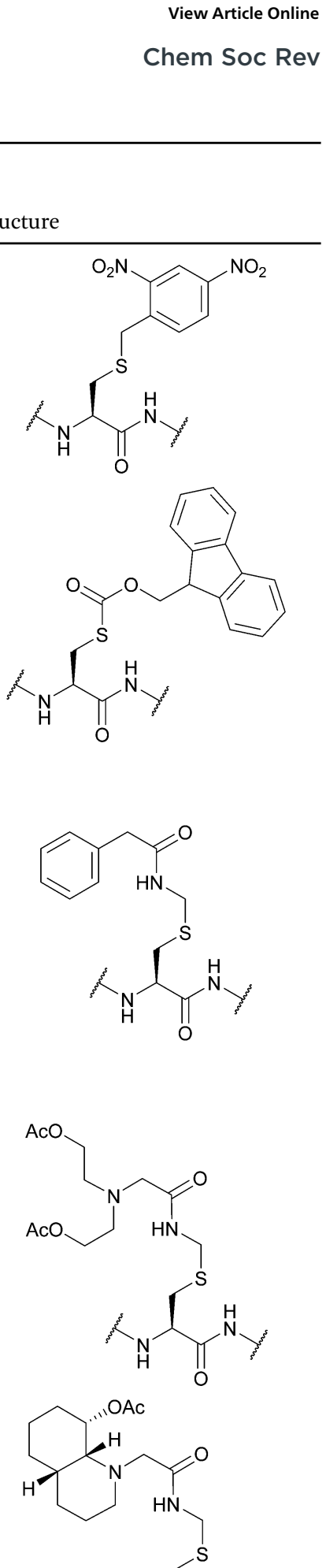

$5 \%$ DIEA in DCM $\left(24 \mathrm{~h}, 25^{\circ} \mathrm{C}\right)^{126}$

$40 \%$ TFA in DCM $\left(24 \mathrm{~h}, 25^{\circ} \mathrm{C}\right)^{126}$

$25 \%$ piperidine in DMF

$\left(24 \mathrm{~h}, 25{ }^{\circ} \mathrm{C}\right)^{126}$

$0.1 \mathrm{M}$ TBAF in DMF

$\left(24 \mathrm{~h}, 25{ }^{\circ} \mathrm{C}\right)^{126}$

$5 \%$ DBU in DMF $\left(24 \mathrm{~h}, 25^{\circ} \mathrm{C}\right)^{126}$

$90 \% \mathrm{HF} /$ anisole or $p$-cresol

$\left(1 \mathrm{~h}, 0{ }^{\circ} \mathrm{C}\right)^{126}$

$90 \% \mathrm{TFA} /$ scavengers

$\left(2 \mathrm{~h}, 25{ }^{\circ} \mathrm{C}\right)^{126}$

Standard Boc SPPS reagents ${ }^{126}$

Standard Fmoc SPPS

reagents ${ }^{126}$

Standard Fmoc SPPS

reagents ${ }^{128}$

Standard Boc SPPS reagents ${ }^{128}$

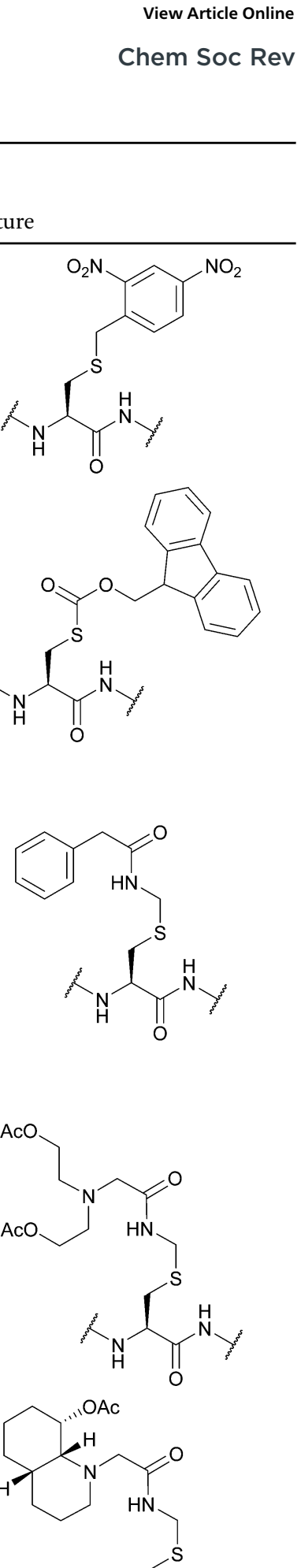

Standard Fmoc SPPS

reagents ${ }^{128}$

Standard Boc SPPS reagents ${ }^{128}$

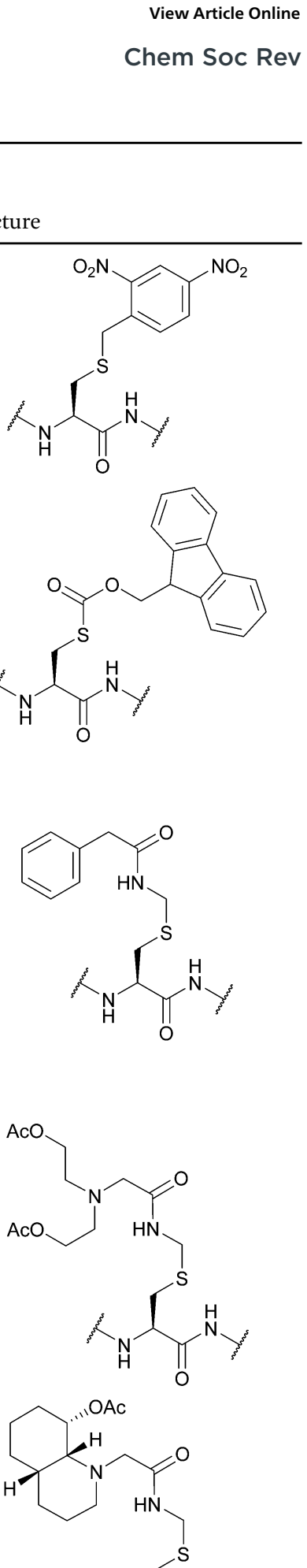

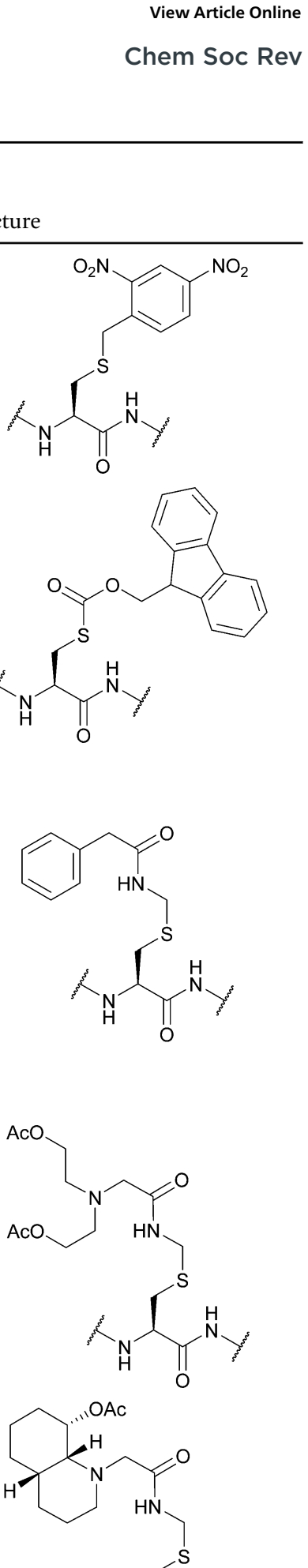

TFA/DCM $\left(24 \mathrm{~h}, 50{ }^{\circ} \mathrm{C}\right)^{129}$ Standard Boc SPPS reagents ${ }^{129}$<smiles>CCNCC</smiles>
To<smiles>C=CCOC(=O)SC[C@H](NC(C)C)C(=O)NC(C)C</smiles> 
Table 1 (continued)

\section{Protecting group, \\ abbreviations and}

location within review

Allyloxy-carbonylaminomethyl (Allocam);

Section 5.7.2

Pd

Lability

Deprotection conditions

Stable to/compatible with

Structure

$\mathrm{Pd}(0)$ cat. $/ \mathrm{Bu}_{3} \mathrm{SnH} / \mathrm{AcOH}$

$(10 \mathrm{~min}, \mathrm{RT})^{130}$

$\mathrm{Pd}(\mathrm{OAc})_{2} / \mathrm{NMM} / \mathrm{AcOH}$ in

DMSO $(2 \mathrm{~h} \text {, disulfide })^{131}$

[N-[2,3,5,6-Tetrafluoro-4( $N^{\prime}$-piperidino)-phenyl], $\mathrm{N}$-allyloxycarbonyl]aminomethyl (Fnam); Section 5.7.3

$S-[N-[2,3,5,6-$ Tetrafluoro-4(phenylthio)-phenyl], $\mathrm{N}$-allyloxycarbonyl]aminomethyl (Fsam); Pd, oxidising agents
Pd, oxidising agents

$\operatorname{Pd}(0)$ cat./allyl scavenger then $\mathrm{AcOH} / \mathrm{BME}^{133}$

$\mathrm{I}_{2}$ and other oxidants ${ }^{133}$

$\operatorname{Pd}(0)$ cat./allyl scavenger

then $\mathrm{AcOH} / \mathrm{BME}^{132}$

Heavy metal salts ${ }^{132}$

$\operatorname{Tl}(\mathrm{TFA})_{3}{ }^{132}$

\section{Acid $^{133}$}

Section 5.7.4

Allyl (Sac); Section 5.7.5

Pd

$\operatorname{Pd}(\text { tppts })_{4}{ }^{134}$

$S$-Propargyl-cysteine (SprC); Section 5.7.6

Succinimide (Suc); Section 5.7.7

$\mathrm{PdCl}_{2}$ and $\mathrm{MgCl}_{2}, 6 \mathrm{M}$ Gdn.HCl/0.2 M phosphate buffer pH 5.5, $37^{\circ} \mathrm{C}$ then DTT $^{136}$
Base $^{133}$

Standard Boc SPPS reagents ${ }^{133}$ Standard Fmoc SPPS reagents ${ }^{133}$

Acid $^{132}$

Base $^{132}$

Standard Boc SPPS reagents ${ }^{132}$ Standard Fmoc SPPS reagents ${ }^{132}$<smiles>C=CCOC(=O)NCSC[C@H](NC(C)C)C(=O)NC(C)C</smiles><smiles>C=CCOC(=O)N(CSC[C@H](NC(C)C)C(=O)NC(C)C)c1c(F)c(F)c(N2CCCCC2)c(F)c1F</smiles><smiles>C=CCOC(=O)N(CSC[C@H](NC(C)C)C(=O)NCC)c1c(F)c(F)c(Sc2ccccc2)c(F)c1F</smiles>

Unnatural amino acid mutagenesis

CuAAC, Sonagashira coupling (alkyne functionality can be used for conjugation without cleavage) ${ }^{135}$

Aqueous solution phase conditions $^{136}$ Desulfurisation conditions ${ }^{136}$<smiles>C#CCSC[C@H](NC(C)C)C(=O)NCC</smiles><smiles>CC(C)NC(=O)[C@H](CS[C@H]1CC(=O)N(C)C1=O)NC(C)C</smiles> 
Table 1 (continued)

Protecting group,
abbreviations and

location within review

Thiazolidine (Thz);

Section 5.8.1

Ninhydrin (Nin);

Section 5.8.2

(n)

2-Nitrobenzyl (oNB);

Section 5.9.1

Light

$h \nu \geq 350 \mathrm{~nm}^{142}$

[7,8-Bis(carboxymethoxy)coumarin-4-yl]methoxycarbonyl (7,8-BCMCMOC);

Section 5.9.2

[7-Bis(carboxymethyl)amino-coumarin-4yl]methoxycarbonyl (BCMACMOC);

Section 5.9.2

$\alpha$-Carboxy-4-methoxy2-nitrobenzyl (CDMNB); Section 5.9.2

$$
\text { Lability }
$$

agents, $\mathrm{Cu}$ $(8 \mathrm{~h})^{138}$ $(0.1 \% \text { TFA })^{140}$ agents

Light $\quad h \nu \geq 325 \mathrm{~nm}^{144}$

Light

$h \nu \geq 402 \mathrm{~nm}^{144}$

Light $\quad h \nu \geq 325 \mathrm{~nm}^{144}$
Deprotection conditions

Stable to/compatible with

Structure

$\begin{array}{lll}\mathrm{N} \text {-terminal } & \mathrm{H}_{2} \mathrm{O}_{2}{ }^{137} \mathrm{I}_{2}{ }^{137} & \mathrm{PdCl}_{2} / \mathrm{H}_{2} \mathrm{O}^{85} \\ \text { Pd, oxidising } & \text { Iodoacetic acid/benzyl } & \text { Standard NCL reagents }\end{array}$

chloride (pH 10-11, RT) ${ }^{137}$

Air/ferric chloride (trace, $\mathrm{pH} 10)^{137}$

Excess methoxyamine at $\mathrm{pH} 4$

Pd(II) and MPAA/TCEP or

GSH/6 M Gdn. $\mathrm{HCl}(\mathrm{pH} \sim 6.5$,

$\left.37{ }^{\circ} \mathrm{C}, 45 \mathrm{~min}\right)^{85,138}$

$\mathrm{CuSO}_{4} /$ sodium ascorbate $/ 5 \mathrm{M}$

$\mathrm{Gdn} \cdot \mathrm{HCl} / \mathrm{HEPPS}$ buffer then

DTT $\left(\mathrm{pH} 7.0,1 \mathrm{~h}, 37^{\circ} \mathrm{C}\right)^{139}$

$20 \mathrm{mM}$ DPDS, 50\% MeCN

N-terminal Excess Cys (pH 7.7, 30 min,

Reducing Cysteine O-methylester/DMF/

DIEA (on resin) ${ }^{141}$

Excess MPS, $\mathrm{pH} 7^{141}$

$10 \% \mathrm{TFA} / \mathrm{H}_{2} \mathrm{O} / \mathrm{Zn}$ dust, $1 \mathrm{~h}^{141}$

Standard Boc SPPS reagents ${ }^{141}$

Standard Fmoc SPPS

reagents ${ }^{143}$

Standard Boc SPPS reagents ${ }^{143}$

TFA $^{144}$

Thiolysis $^{144}$

$\mathrm{TFA}^{144}$

Thiolysis $^{144}$

$\mathrm{TFA}^{144}$

Thiolysis $^{144}$

Piperidine ${ }^{144}$<smiles>[SiH3][SiH3]</smiles><smiles>CC(C)NC(=O)[C@H](CS)NC(C)C</smiles><smiles>CC(C)NC(=O)[C@H](CSC(=O)OCc1cc(=O)oc2cc(N(CC(=O)O)CC(=O)O)ccc12)NC(C)C</smiles><smiles>CCNC(CSCc1ccccc1[N+](=O)[O-])C(=O)NC(C)CC</smiles><smiles>CCNC(CSCc1ccccc1[N+](=O)[O-])C(=O)NC(C)CC</smiles><smiles>CCNC(CSCc1ccccc1[N+](=O)[O-])C(=O)NC(C)C(=O)NC(C)C</smiles><smiles>CCNC(CSCc1ccccc1[N+](=O)[O-])C(=O)NC(C)CC(=O)C1CSC2(N1)C(=O)c1ccccc1C2=O</smiles>

(n)<smiles>COc1ccc([C@H](SC[C@H](NC(C)C)C(=O)O)C(=O)NC(C)C)c([N+](=O)[O-])c1</smiles> 
Table 1 (continued)

Protecting group,

abbreviations and

location within review

2-Nitroveratryl

6-Nitroveratryl

4,5-Dimethoxy-2-

nitrobenzyl ( $\circ \mathrm{NV}, \mathrm{DMNB})$

Section 5.9 .3

\section{6-Bromo-7-hydroxy- coumarin (Bhc);}

Section 5.9.4

Light

$h \nu=365 \mathrm{~nm}$ in photolysis

buffer (1 mM DTT in $50 \mathrm{mM}$ $\mathrm{PB}, \mathrm{pH} 7.2)^{145}$

Standard Fmoc SPPS reagents ${ }^{145}$

10\% TFMSA/TFA/excess dipyridine disulfide ${ }^{142}$ Standard Fmoc SPPS reagents ${ }^{142}$

$h \nu=350 \mathrm{~nm}(30 \mathrm{~min})^{142}$

Nitrodibenzofuran (NDBF); Section 5.9.5

Light

$h \nu=365 \mathrm{~nm}^{145}$

$h \nu=365 \mathrm{~nm}^{146}$

methylcoumarin (mBhc);

Section 5.9.6

Light

Light

Methoxy-nitrodibenzofuran

(OMe-NDBF); Section 5.9.7

$h \nu \geq 350 \mathrm{~nm}^{147}$

$\mathrm{Zn} / \mathrm{AcOH}$, then $\mathrm{I}_{2}$

(in solution) ${ }^{111}$

$\mathrm{SnCl}_{2} / \mathrm{HCl}$, then $\mathrm{I}_{2}$

para-Nitrobenzyl ( $p \mathrm{NB})$;

Reducing

(on resin) ${ }^{111}$

Section 5.10.1

agents

Standard Fmoc SPPS
Excess CAN/Hopkins

reagent ${ }^{111}$

$\mathrm{H}_{2}, \mathrm{Pd} / \mathrm{C}$, then oxidant ${ }^{111}$
Standard Fmoc SPPS reagents ${ }^{145}$ reagents ${ }^{146}$

Standard Fmoc SPPS reagents ${ }^{147}$

$\mathrm{HF} / p$-cresol $\left(9: 1,1 \mathrm{~h}, 0{ }^{\circ} \mathrm{C}\right)^{111}$ $\mathrm{TFA}^{111}$

$\mathrm{I}_{2} / \mathrm{AcOH} / 2 \mathrm{M} \mathrm{HCl}^{111}$

Standard Boc SPPS reagents ${ }^{111}$
Structure<smiles>COc1cc(CSC[C@H](NC(C)C)C(=O)NC(C)C)c([N+](=O)[O-])cc1OC</smiles><smiles>CC(C)NC(=O)[C@H](CSCc1cc(=O)oc2cc(O)c(Br)cc12)NC(C)C</smiles><smiles>CC(C)NC(=O)[C@H](CS[C@H](C)c1cc2c(cc1[N+](=O)[O-])oc1ccccc12)NC(C)C</smiles><smiles>Cc1c(CSC[C@H](NC(C)C)C(=O)NC(C)C)c2cc(Br)c(O)cc2oc1=O</smiles><smiles>COc1ccc2c(c1)oc1cc([N+](=O)[O-])c([C@H](C)SC[C@H](NC(C)C)C(=O)NC(C)C)cc12</smiles><smiles>CC(C)NC(=O)[C@H](CSCc1ccc([N+](=O)[O-])cc1)NC(C)C</smiles> 
Table 1 (continued)

Protecting group,

abbreviations and

location within review

(a)

Carbomethoxysulfenyl

(Scm); Section 5.10.2

Reducing

agents

DTT $^{148}$

( $N^{\prime}$-Methyl- $N^{\prime}$ -

phenylcarbamoyl)sulfenyl

(Snm); Section 5.10.3

4-Picolyl; Section 5.10.4

Reducing agents

Sulfonic acid/sulfonyl $\left(\mathrm{SO}_{3} \mathrm{H} / \mathrm{SO}_{2} \mathrm{R}\right)$; Section 5.10.5

3-Nitro-2-pyridinesulfenyl (Npys); Section 5.10.6

Reducing agents

Reducing

5-Nitro-2-pyridinesulfenyl (5-Nyps); Section 5.10.7

Thiols $^{158}$

$$
\begin{aligned}
& \text { Thiols }^{159} \\
& \text { Phosphines }{ }^{114} \\
& \text { TFA/DTNP/thioanis } \\
& \text { Zn/AcOH (aq. })^{160} \\
& \text { TFA }\left(1 \mathrm{~h}, 50{ }^{\circ}\right)^{160}
\end{aligned}
$$$$
\text { TFA/DTNP/thioanisole }{ }^{80}
$$

Reducing Section 5.10 .8 agents
Aliphatic thiols ${ }^{154}$
Tertiary phosphine/ $\mathrm{H}_{2} \mathrm{O}^{155,156}$

TFA (24 h, RT) $)^{155}$

HF $(1 \mathrm{~h}, \mathrm{RT})^{15}$

$4 \mathrm{M} \mathrm{HCl} /$ dioxane $(24 \mathrm{~h})^{155}$ DCM $^{157} \mathrm{DMF}^{157} \mathrm{MeOH}^{157}$ $N, N$-Dimethylacetaminde ${ }^{157}$ $N$-Methylpyrrolidone $e^{157}$ Trifluoroethanol ${ }^{157}$ Pentafluorophenol ${ }^{157}$ Standard Boc SPPS reagents ${ }^{155}$

Standard Boc SPPS reagents (see Nyps) ${ }^{158}$

Acid $^{159}$

Base $^{159}$

TFA/DNTP ${ }^{80}$

Standard Fmoc SPPS reagents $^{74}$
$N$-Methyl-phenacyloxycarbamidomethyl (Pocam); Section 5.10 .9
Reducing agents, acid
TFA $\left(4 \mathrm{~h}, 4{ }^{\circ} \mathrm{C}\right)^{160}$ Standard Fmoc SPPS reagents ${ }^{160}$
Structure<smiles>COC(=O)SS</smiles><smiles>CCC(NC(C)C)C(=O)NC(C)C</smiles><smiles>CC(C)NC(=O)C(CSSC(=O)N(C)c1ccccc1)NC(C)C</smiles><smiles>CC(C)NC(=O)C(CSCc1ccncc1)NC(C)C</smiles><smiles>[R]OS(C)(=O)=O</smiles><smiles>CC(C)NC(=O)C(CS)NC(C)C</smiles><smiles>CC(C)NC(CSSc1ncccc1[N+](=O)[O-])C(=O)NC(C)(C)C</smiles><smiles>CC(C)NC(CSSc1ccc([N+](=O)[O-])cn1)C(=O)NC(C)(C)C</smiles><smiles>CCNC(=O)C(CSSC(C)(C)C)NC(C)C</smiles><smiles>CC(C)NC(=O)C(CSCN(C)C(=O)OCC(=O)c1ccccc1)NC(C)C</smiles> 
Table 1 (continued)

Protecting group,

abbreviations and location within review

Lability

Deprotection conditions

TCEP in PBS pH 7.4
$37^{\circ} \mathrm{C}^{114}$

$S$-Iso-Propyl (SiPr);

Section 5.10.11

Dimethoxyphenylthio

( $S$-Dmp); Section 5.10.12

2,4,6-Trimethoxyphenylthio ( $S$-Tmp); Section 5.10.12

Reducing agents
2-Methyloxolane-3-thiol (MOT); Section 5.10.13 BME/DMF or $5 \%$ DTT/DMF
2-Pyridinesulfenyl ( $S$-Pyr); Section 5.10.14
Reducing agents
4,4-Bis(dimethylsulfinyl)benzhydryl (Msbh); Section 5.11.1
Reducing agents

Reducing agents

$\begin{array}{ll} & \text { BME in DMF }(1: 4), 0.1 \mathrm{M} \\ & \text { DIEA }^{165} \\ & 20 \text { equiv. DTT, MeCN/DIEA/ } \\ \text { Reducing } & \mathrm{H}_{2} \mathrm{O}(90: 5: 5)^{165} \\ \text { agents } & 5 \text { equiv. DTT } \times 3, \text { DMF/DIEA/ } \\ & \mathrm{H}_{2} \mathrm{O}(95: 2.5: 2.5)^{165}\end{array}$

BME in DMF (1:4), $0.1 \mathrm{M}$ DIEA $^{165}$

20 equiv. DTT, MeCN/DIEA/

NMM $(0.1 \mathrm{M})$ then either $20 \%$ $(5 \mathrm{~min})^{163}$

DABDT, DIEA $/ \mathrm{H}_{2} \mathrm{O} / \mathrm{MeCN} \quad 95 \%$ TFA $(1 \mathrm{~h}, \mathrm{RT})^{163}$ $(3: 3: 94)^{164}$

NMM $(0.1 \mathrm{M})$ then either $20 \%$ $\mathrm{BME} / \mathrm{DMF}$ or $5 \% \mathrm{DTT} / \mathrm{DMF}$ $(5 \mathrm{~min})^{163}$

$\mathrm{H}_{2} \mathrm{O}(90: 5: 5)^{165}$

Reducing 5 equiv. DTT $\times 3, \mathrm{DMF} / \mathrm{DIEA} /$ $\mathrm{H}_{2} \mathrm{O}(95: 2.5: 2.5)^{165}$

Standard Fmoc SPPS
reagents

95\% TFA $(1 \mathrm{~h}, \mathrm{RT})^{163}$

Standard Fmoc SPPS

reagents ${ }^{163}$

Standard Fmoc SPPS

reagents ${ }^{165}$

Standard Fmoc SPPS

reagents ${ }^{165}$
Safety-catch
Thiols $^{4}$

Acid (TFA, HF) ${ }^{166}$

Oxidants ${ }^{166}$

Reductants ${ }^{166}$

Standard Boc SPPS reagents ${ }^{166}$
Structure<smiles>CC(C)NC(=O)C(CSCC(=O)c1ccccc1)NC(C)C</smiles>

Standard Fmoc SPPS

reagents ${ }^{114}$

$20 \%$ piperidine in DMF $(4 \mathrm{~h})^{163}$

1 M TFMSA in TFA-anisole $\left(10: 1,2 \mathrm{~h}, 0{ }^{\circ} \mathrm{C}\right)^{4}$

Standard Boc SPPS reagents ${ }^{4}$<smiles>CCNC(=O)[C@H](CSSC(C)C)NC(C)C</smiles><smiles>CCNC(=O)[C@H](CSSc1ccc(OC)cc1OC)NC(C)C</smiles><smiles>CCCNC(=O)[C@H](CSSc1c(OC)cc(OC)cc1OC)NC(C)C</smiles><smiles>CC(C)C(C)SS</smiles><smiles>CC(C)NC(=O)[C@H](CS)NC(C)C</smiles><smiles>CC(C)NC(=O)[C@H](CSSC1CCOC1C)NC(C)C</smiles><smiles>CC(C)NC(=O)[C@H](CSSc1ccccn1)NC(C)C</smiles><smiles>CC(C)NC(=O)[C@H](CSC(c1ccc(S(C)=O)cc1)c1ccc(S(C)=O)cc1)NC(C)C</smiles> 
a) Benzyl

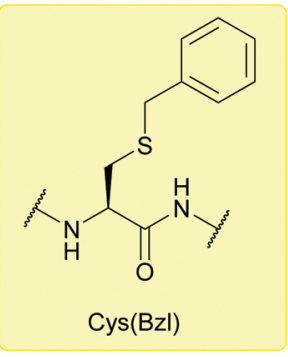

b) Oxytocin synthesis<smiles>CC(C)(C)OC(=O)Nc1ccc(C(=O)O)cc1SCC(=O)O</smiles>

i) Tetraethyl pyrophosphate

$\mathrm{H}_{2} \mathrm{~N} \rightarrow \mathbf{Q}(\mathbf{C})\left(\mathbf{C}-\mathrm{CONH}_{2}\right.$

ii) $\mathrm{Na} / \mathrm{NH}_{3}$ (liq.) iii) $\mathrm{H}_{2} \mathrm{O}, \mathrm{pH} 6.5$ (air oxidation)

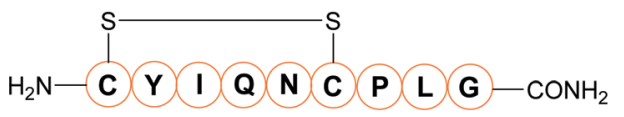

Oxytocin

Fig. 5 (a) Cys thiol protection with the benzyl (Bn/Bzl) protecting group (b) the synthesis of oxytocin using Cys(Bzl). removing Bzl with $\mathrm{Na} / \mathrm{NH}_{3}$ (liq.) have been described as far back as $1930,{ }^{67}$ and Bzl can also be removed by treatment with $\mathrm{HF}$ at $25{ }^{\circ} \mathrm{C},{ }^{68}$ or with strong Lewis acids (e.g. TMSBr) in TFA-thioanisole. ${ }^{69}$ However, these removal methods have a number of significant issues associated with them. For example, using $\mathrm{Na} / \mathrm{NH}_{3}$ (liq.) cleaves existing disulfide bonds alongside Bzl removal. ${ }^{70} \mathrm{HF}$ gas, in particular, is expensive, not widely available, and highly toxic. TMSBr been reported to undergo side reactions with peptides and has a relatively poor solubility in diethyl ether $\left(\mathrm{Et}_{2} \mathrm{O}\right.$, a commonly used solvent for precipitation of a synthesised peptide once cleaved from the resin). TMSBr can thus precipitate with the peptide instead of being removed resulting in issues with handling following lyophilisation. ${ }^{90,170}$ Considering this, it is likely that Bzl will see diminishing use as a Cys protecting group in routine peptide synthesis, as it is superseded by newer protecting groups, benzyl-based or otherwise, that do not require such harsh conditions for removal. ${ }^{4,78}$

\subsection{Acid-labile protecting groups}

5.2.1 Trityl (Trt). In 1962 the trityl (Trt) group (Fig. 6a) was proposed as an alternative Cys protecting group to protecting groups such as Bzl. ${ }^{70}$ The key difference, in this case, was that cleavage of Trt could be accomplished without reduction of existing disulfide bonds which was a side effect of $\mathrm{Na} / \mathrm{NH}_{3}$ (liq.) treatment. Originally, metal salts such as $\mathrm{Ag}$ (I) or $\mathrm{Hg}$ (II) were used to remove the group; provided the amino and carboxyl groups in the peptide were protected, cleavage of the disulfide bond was repressed, enabling the synthesis of unsymmetrical

\section{a) Trityl}

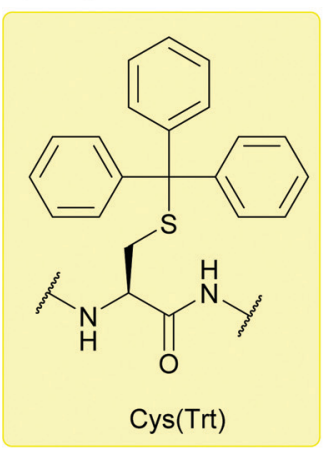

b) Marcocylic Cys(Trt) peptides

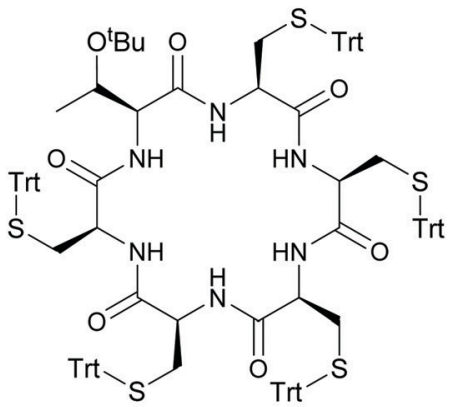

c) Regioselective disulfide formation via post-synthetic tritylation strategy
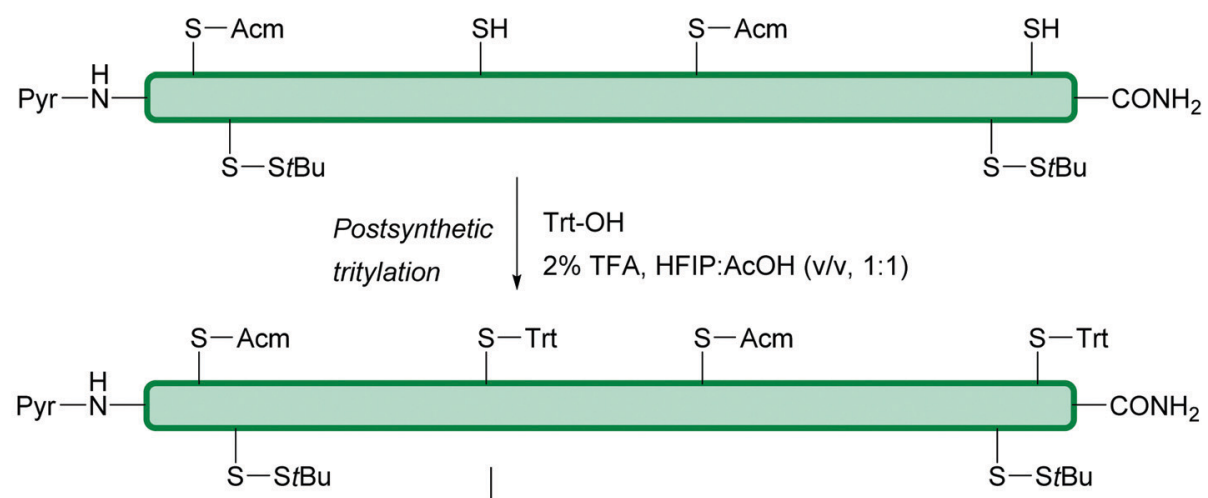

Orthogonal protection/deprotection Disulfide formation

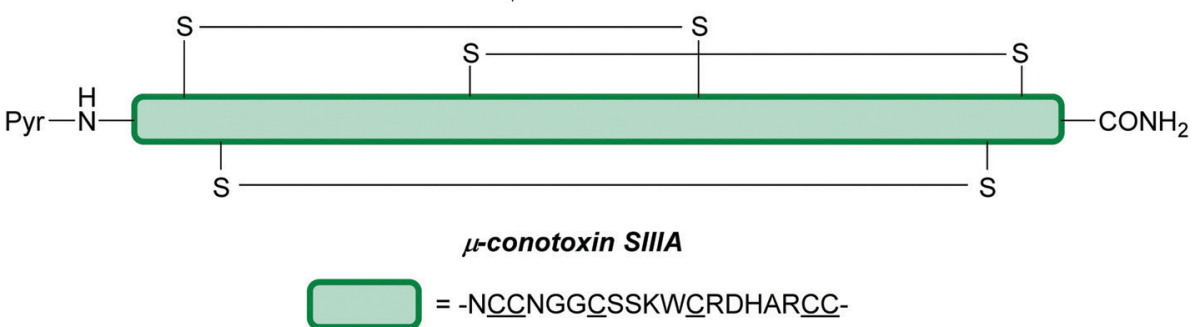

Fig. 6 (a) Cys thiol protection with the trityl (Trt) protecting group. Examples of Cys(Trt) use include (b) macrocyclic peptide synthesis and (c) regioselective disulfide formation in $\mu$-conotoxin SIIIA via a post-synthetic tritylation strategy. 
Cys-containing peptides containing two or more disulfide bonds. ${ }^{70}$ Additionally, acids such as $\mathrm{HBr} / \mathrm{AcOH}$ and TFA were noted to cleave the Trt group without affecting existing disulfide bonds. ${ }^{70}$

Should the desired product of Trt cleavage be a new disulfide bond, oxidising agents such as $\mathrm{I}_{2}$ may be used. ${ }^{4}$ The rate of Trt removal and subsequent disulfide formation by $\mathrm{I}_{2}$ has previously been to shown to depend on the choice of solvent. ${ }^{171}$ It is common to remove Trt using weak acids (e.g. $\mathrm{HBF}_{4}$ or TFA) in the presence of scavengers such as triisopropylsilane (TIS) or triethylsilane (TES); these scavengers prevent the released Trt cations from adding back onto the synthesised peptide during cleavage and isolation. ${ }^{172}$ This method of Trt removal renders Trt orthogonal to other common protecting groups such as Acm or $t \mathrm{Bu}$, as shown in the regioselective synthesis of human insulin, via sequential disulfide formation. ${ }^{72}$ Other applications of Cys(Trt) in SPPS include synthesis of human relaxin, ${ }^{173}$ and $\alpha$-melanocyte stimulating hormone ( $\alpha$-MSH) analogues. ${ }^{174}$ Additionally, macrocyclic peptides displaying in vitro anti-malarial properties have also been synthesised using Cys(Trt); in this example, the Trt protecting group is retained rather than removed in the final bioactive product (Fig. 6b). ${ }^{175} \mathrm{Cys}(\mathrm{Trt}$ ) has also very recently been reported to undergo complete deprotection in model peptides when treated with $\mathrm{CuSO}_{4}$ and cysteamine in aqueous buffered conditions. ${ }^{73}$

Cys(Trt) is routinely used in Fmoc SPPS. It is worth noting that Trt is highly hydrophobic and that the Trt cation-scavenger adducts formed during deprotection may not be removed completely during final TFA cleavage however. The presence of Trt can then mask the quality of the crude peptide due to its high UV absorbance. ${ }^{103}$ To avoid incomplete detritylation, typical cleavage cocktails of $>90 \%$ TFA with TIS/TES (typically ca. 5\%) can be used. These conditions can, however, cause the reduction of the indole ring of Trp. ${ }^{71,172}$ Due to its lability to TFA, Trt is removed during cleavage of acid-labile resins (as commonly seen in Fmoc SPPS). It is still possible to use Trt as a protecting group when forming disulfide bonds in solution post-cleavage from the resin, however, the deprotected residues must be re-tritylated post-synthetically. This was exemplified in the regioselective syntheses of $\mu$-conotoxin SIIIA (Fig. 6c) and human hepcidin, using combinations of StBu, Trt, Meb/Mob and Acm. ${ }^{74}$
5.2.2 Diphenylmethyl (Dpm). The diphenylmethyl (Dpm) group (Fig. 7a) was introduced as a Bzl replacement by Zervas and Photaki alongside the Trt group in $1962 .{ }^{70}$ It is also sometimes referred to as the benzhydryl (Bzh/Bh) protecting group in the literature, ${ }^{176,177}$ and has featured in Boc SPPS of peptides such as oxytoceine (which is oxidised to oxytocin post cleavage/ deprotection). ${ }^{178}$ The use of Dpm in Fmoc SPPS was first reported in 2012, where it was suggested as a replacement for the acid-labile Mob protecting group. ${ }^{75} \mathrm{Dpm}$ is stable to low concentrations of TFA $(<25 \%)$ but can be cleaved with higher concentrations - up to 90\% TFA in DCM (using $2.5 \%$ TIS and $2.5 \% \mathrm{H}_{2} \mathrm{O}$ as scavengers) is required for full removal. Due to its acid lability profile, Dpm is orthogonal to Trt and Mmt and fills a niche between groups that are labile to weak acids, e.g. Trt, and groups that are only removed using strong acids such as $\mathrm{HF}$, e.g. Meb. ${ }^{75,105}$ Additionally, Cys racemisation (a known side effect during incorporation when using phosphonium and uronium salt-based coupling reagents) is attenuated considerably when using Dpm compared to Trt or Bzl: racemisation levels of $1.2 \%, 8.0 \%$ and $5.3 \%$ are seen under conventional Fmoc SPPS conditions, respectively. ${ }^{106}$ The acid stability profile of Dpm was demonstrated in the synthesis of $\alpha$-conotoxin ImI (in conjunction with Trt), along with synthesis of the hinge region of human immunoglobulin G1 (IgG1, 225-232/225'-232', in conjunction with Mmt, Fig. 7b).

5.2.3 Tetrahydropyranyl (Thp). The tetrahydropyranyl (Thp) group (Fig. 8) was first introduced as a Cys protecting group in 1958, when it was found to be slowly removed by $\mathrm{Na} / \mathrm{NH}_{3}$ (liq.) reduction yet rapidly removed by aqueous $\mathrm{AgNO}_{3}$ at $0{ }^{\circ} \mathrm{C}$. ${ }^{76} \mathrm{Thp}$ was further established as a Cys protecting group in 2015, where its use in Fmoc SPPS was discussed. ${ }^{77}$ Thp is stable in mildly acidic conditions, but its acid-lability is strongly increased in the presence of TIS. It can be removed under conventional cleavage conditions, e.g. (i) $10 \%$ TFA and $2.5 \%$ TIS in DCM, (ii) $95 \%$ TFA and $2.5 \%$ TIS in $\mathrm{H}_{2} \mathrm{O}$ or (iii) $0.1 \mathrm{M} \mathrm{HCl}$ and HFIP-TIS (99:1). Cys(Thp)-protected peptides exist as a diastereomeric mixture; however, once cleaved in concentrated TFA with a scavenger $(e . g$. 95\% TFA, 2.5\% TIS in DCM), a single pure product is obtained. ${ }^{77}$ Thp protected tripeptides displayed greater solubility in $\mathrm{H}_{2} \mathrm{O}$ / MeCN solvent systems (as assessed by reverse-phase HPLC) than

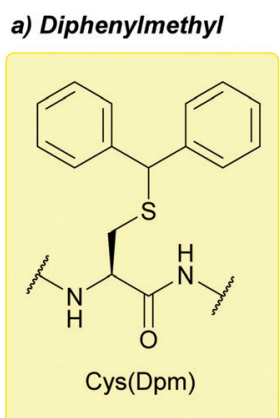

$$
\begin{aligned}
& \text { b) IgG1 hinge } \\
& \text { fragment synthesis }
\end{aligned}
$$

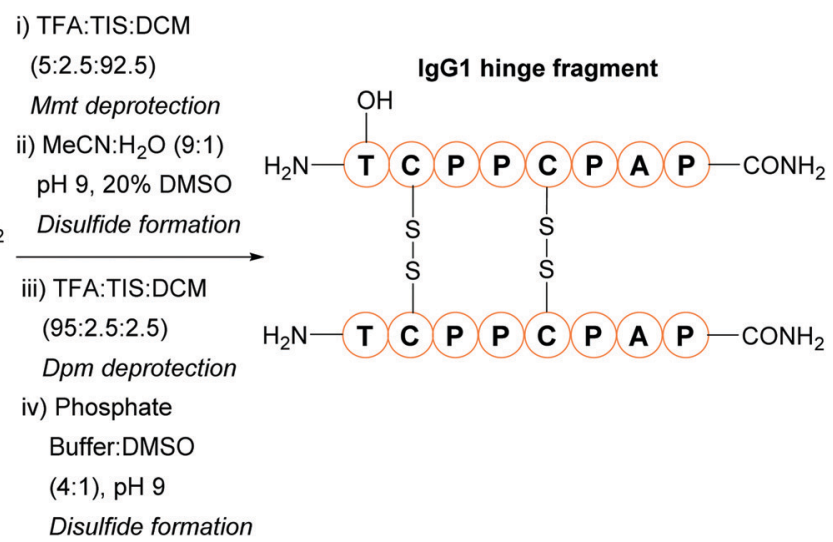

Fig. 7 (a) Cys thiol protection with the diphenylmethyl (Dpm) protecting group (b) synthesis of the IgG1 hinge region fragment using Cys(Dpm). 


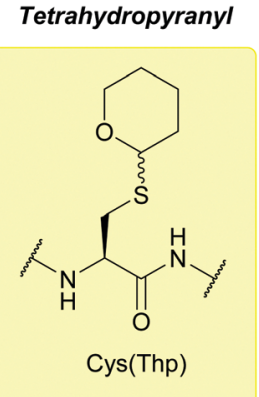

Fig. 8 Cys thiol protection with the tetrahydropyranyl (Thp) protecting group.

either Trt or Dpm protected tripeptides. Racemisation is also decreased compared to Trt, Dpm or StBu, and fewer side products, e.g. C-terminal 3-(1-piperidinyl)alanine adducts, are observed. ${ }^{77}$ More recently, Thp has been explored as a protecting group for additional amino acid residues such as Ser and Thr. ${ }^{179}$
5.2.4 tert-Butyl ( $t$ Bu). The use of the tert-butyl ( $t \mathrm{Bu})$ group to protect Cys (Fig. 9a) was first proposed in $1962 .{ }^{180}$ It was then later discovered that 2-(nitrophenyl)sulfenyl chloride ( $\mathrm{NpsCl})(2 \mathrm{~h}$, $\mathrm{RT}$ ) could cleave the $t \mathrm{Bu}$ group. This could then be followed by treatment with $\mathrm{NaBH}_{4}, 2$-mercaptoethanol (BME) or thioglycolic acid to obtain the free thiol. It should be noted that these conditions will also cleave Acm. ${ }^{78}$ The $\mathrm{Cys}(t \mathrm{Bu})$ protecting group has been shown to be stable to oxidation by $\mathrm{I}_{2}{ }^{72}$ and neat TFA treatment. ${ }^{86}$ Cleavage of $t \mathrm{Bu}$ can be achieved with HF using anisole as a scavenger ${ }^{79}$ (the group remains intact if $m$-cresol is used as the scavenger instead), ${ }^{72}$ and TFA in the presence of $2,2^{\prime}$ dithiobis(5-nitropyridine) (DTNP). ${ }^{80} t \mathrm{Bu}$ may also be cleaved using $\mathrm{Hg}(\mathrm{OAc})_{2}$ in cold TFA-anisole (alternatively, $\mathrm{Hg}(\mathrm{TFA})_{3}$ in AcOH (aq.)). ${ }^{81}$ To form the disulfide, cleavage may be performed using silyl chloride-sulfoxide in TFA, ${ }^{82} \mathrm{Tl}(\mathrm{TFA})_{3}{ }^{83}$ or DMSO in TFA (with DMSO acting as an oxidant). The latter method has been used for the regioselective syntheses of various analogues of human insulin containing four disulfide bonds, in conjunction

a) tert-Butyl

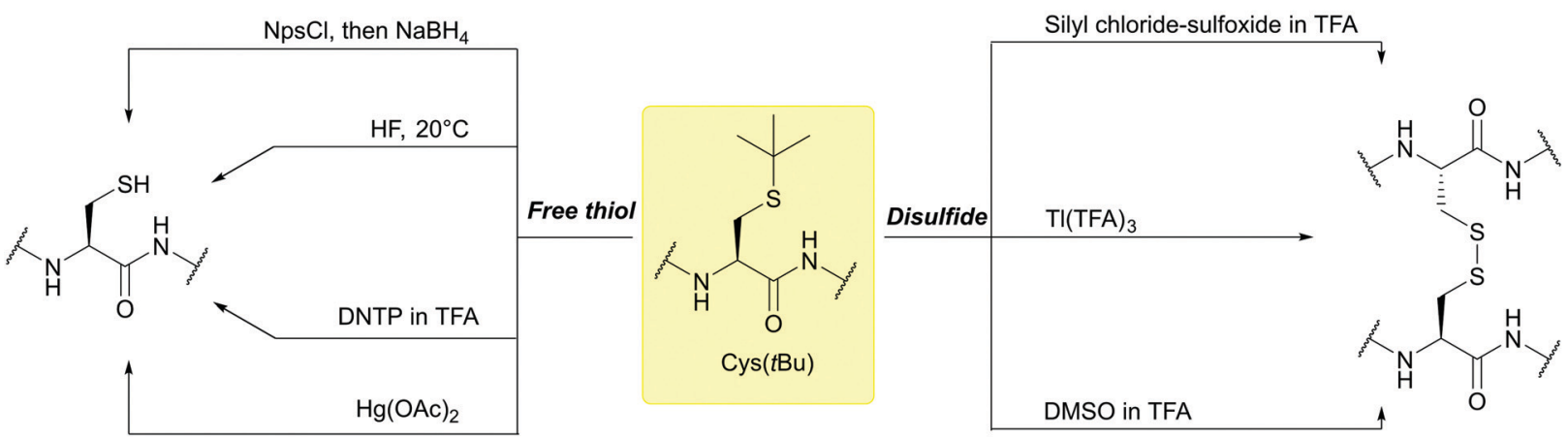

b) Ubiquitinated histone $\mathrm{H} 2 \mathrm{~A}$ activity-based probe synthesis
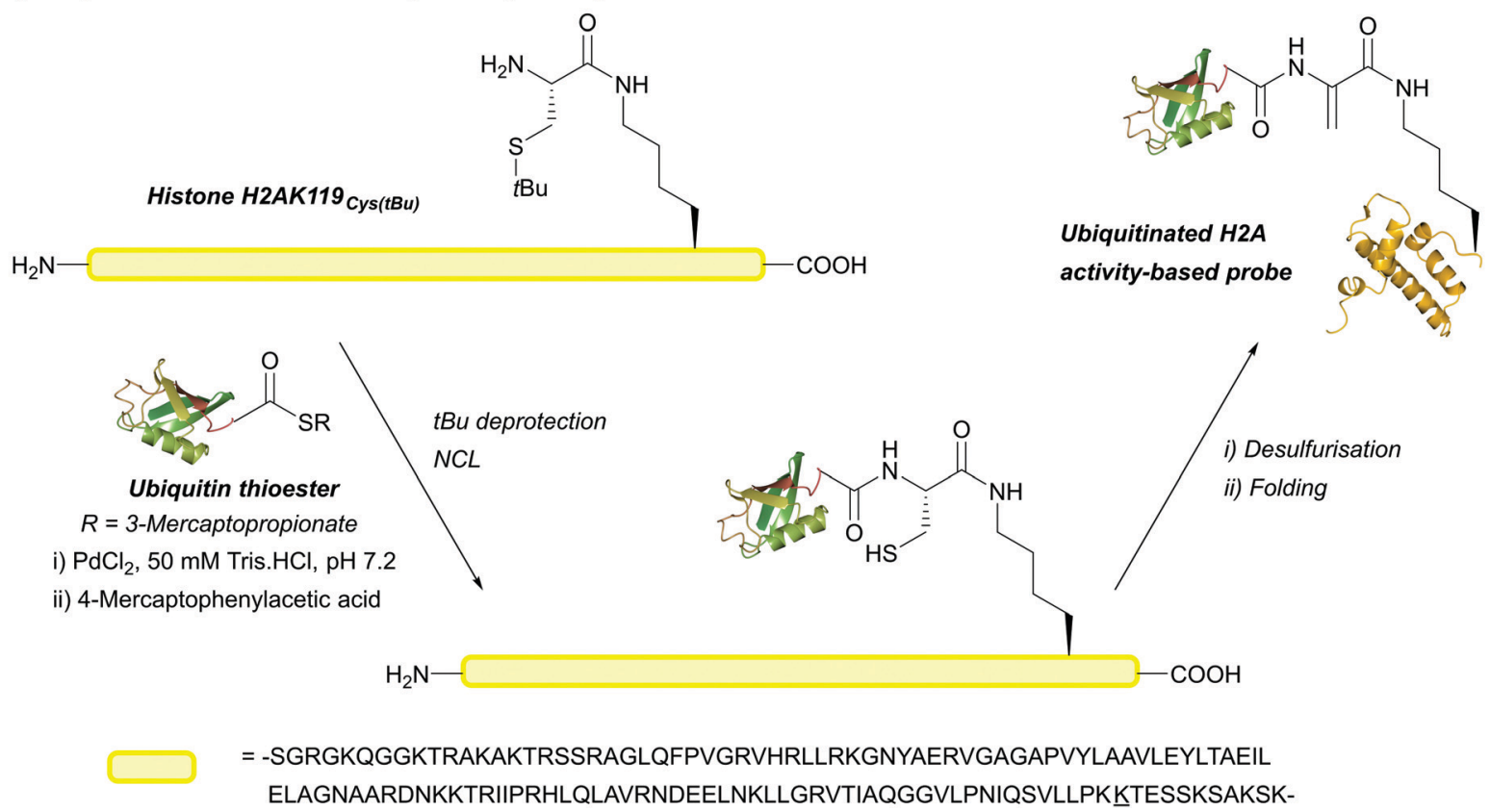

Fig. 9 (a) Cys thiol protection with the tert-butyl (tBu) protecting group and different methods of deprotection to give the corresponding Cys or cystine. (b) Synthesis of a ubiquitinated histone $\mathrm{H} 2 \mathrm{~A}$ activity-based probe using $\mathrm{Cys}(t \mathrm{Bu})$ and $\mathrm{PdCl}_{2}$ for deprotection under aqueous conditions. 
with Trt, Acm and $\mathrm{Mmt}^{.84}$ If orthogonality to Meb is desired, DMSO/TFA may also be used for deprotection - $t \mathrm{Bu}$ is cleaved in DMSO/TFA at room temperature, whilst higher temperatures $\left(45{ }^{\circ} \mathrm{C}\right)$ are required to cleave Meb. This has been used in the regioselective synthesis of an $\alpha$-conotoxin dimer peptide with four disulfide bonds. Deprotection and oxidation were performed simultaneously in two one-pot procedures (the second procedure involved the Trt and Acm groups). ${ }^{88} t \mathrm{Bu}$ is stable to AgOTf in TFA, making it orthogonal to Mob and Acm under those conditions, ${ }^{87}$ as well as to alkaline ester hydrolysis, hydrazine, and $\mathrm{Na} / \mathrm{NH}_{3}$ (liq.). ${ }^{78}$

The above methods of deprotection are harsh, ${ }^{128}$ and often result in the formation of side products and low yields. ${ }^{166}$ Additionally, incubation in neat TFA causes $\sim 20 \%$ deprotection. $^{86}$ In $2018, \mathrm{PdCl}_{2}$ in a $50 \mathrm{mM}$ Tris or urea buffer at $37^{\circ} \mathrm{C}$ was shown to cleave $t \mathrm{Bu}$, providing a much milder way to remove the protecting group. ${ }^{85}$ This is significant for $t \mathrm{Bu}$, as the harsh conditions required for deprotection can hinder its practical use. $t \mathrm{Bu}$ is not removed by $[\mathrm{Pd}(\text { allyl }) \mathrm{Cl}]_{2}$, making it orthogonal to Thz and Acm under those conditions. This method of removal was successfully used to synthesise an activity-based probe of ubiquitinated histone $\mathrm{H} 2 \mathrm{~A}$ (Fig. 9b). ${ }^{85}$

5.2.5 4-Methoxybenzyl (Mob). 4-Methoxybenzyl (Mob, MBzl) was introduced in 1964 as a Cys protecting group (Fig. 10a) that is labile to strong acids - originally boiling TFA, ${ }^{89}$ but also $\mathrm{HF}^{79}$ or TFA-thioanisole plus an electrophilic disulfide (DTNP, DTP). ${ }^{80,89,90}$ Mob can also be removed by heavy metal salts, e.g. $\mathrm{Hg}(\mathrm{TFA})_{2}{ }^{81}$ or AgOTf in TFA/anisole, followed by DTT to obtain the free thiol. ${ }^{87}$ $\mathrm{Tl}(\mathrm{TFA})_{3}$ can also be used for deprotection ${ }^{83}$ Additionally, AgOTf cannot cleave Meb, making Mob and Meb orthogonal under this treatment. ${ }^{87}$ Mob is also stable to $\mathrm{HBr}^{89}$ Compared to Meb, the Mob group is much more acid-labile - it is at least 2700 times less stable in $50 \%$ TFA. $^{91}$ Mob is however mostly stable to TFA without any scavengers at room temperature. At elevated temperatures and extended reaction times $\left(12 \mathrm{~h}, 37^{\circ} \mathrm{C}\right)$, in the presence of TIS, complete deprotection has been noted (with the resulting mixture existing as both the free thiol and the disulfide). ${ }^{86}$ The conditions typically required for full removal of Mob are harsh: relatively high concentrations of TFA (or HF), long reaction times and high temperatures. ${ }^{75}$ Additionally, Mob is slightly labile to TFA when treated for extended periods - $\sim 10 \%$ deprotection has been observed following treatment for $2.5 \mathrm{~h}$ at $0{ }^{\circ} \mathrm{C}$. Partial conversion to $t \mathrm{Bu}$ can then occur, as Boc-derived $t \mathrm{Bu}$ cations may attack the Cys residue following partial Mob cleavage. ${ }^{93}$ This means that Mob may not be suitable for Boc SPPS if large peptides that require repeated TFA treatment are being synthesised. ${ }^{92}$ Nevertheless, Mob has been demonstrated as a protecting group in the Boc solution phase synthesis of peptides, including oxytocin, urotensin II, and human calcintonin. ${ }^{83} \mathrm{Cys}(\mathrm{Mob})$ has also been incorporated into tripeptide benzyl esters for use as potential DNA intercalators, with the protecting group intact in the final product. ${ }^{181} \mathrm{Cys}(\mathrm{Mob})$ is also compatible with Fmoc SPPS, as demonstrated in the synthesis of methylene thioacetal human insulin analogue (SCS-Ins), ${ }^{182}$ and of LaIT2, a $\beta-\mathrm{KTx}^{183}$ peptide that is found in Liocheles australasiae scorpion venom, and displays antimicrobial activity. ${ }^{184}$

Cys(Mob) can undergo oxidation to give the corresponding Mob-protected sulfone, $\operatorname{Cys}(\operatorname{Mob}(\mathrm{O}))$ (Fig. 10b) with either $\mathrm{NaBO}_{3}{ }^{185}$ or $\mathrm{H}_{2} \mathrm{O}_{2} \cdot{ }^{186} \mathrm{Cys}(\mathrm{Mob}(\mathrm{O}))$ can in turn be incorporated into peptides via Fmoc-SPPS. Deprotection with TfOH:TFA: $\mathrm{H}_{2} \mathrm{O}$ $(50: 45: 5)$ leads to removal of the Mob protecting group, generating peptides bearing Cys sulfinic acid, a known PTM of Cys-containing proteins that can regulate protein function. ${ }^{186}$ Additionally, Cys(Mob(O)) can be used for disulfide formation via sulfoxidedirected disulfide reactions ${ }^{187}$ in an $\mathrm{I}_{2}$-free manner $\left(\mathrm{I}_{2}\right.$ can otherwise oxidise amino acids such as Trp). ${ }^{188}$ Example syntheses include oxytocin, ${ }^{189}$ chicken calcitonin-gene-related peptide (cCGRP), ${ }^{190}$ and human insulin-like peptide-6 (INSL-6) (Fig. 10c). ${ }^{188}$

5.2.6 3,4-Dimethylbenzyl (DMB). The 3,4-dimethylbenzyl (DMB) Cys protecting group (Fig. 10d) was developed in 1973 as an alternative to Mob for the synthesis of large peptides using Boc SPPS. ${ }^{92}$ DMB is noticeably more stable to TFA than Mob, with only $0.2 \%$ deprotection observed under $50 \%$ TFA in DCM for $23 \mathrm{~h}$ at $24{ }^{\circ} \mathrm{C}$. The group is, however, easily cleaved

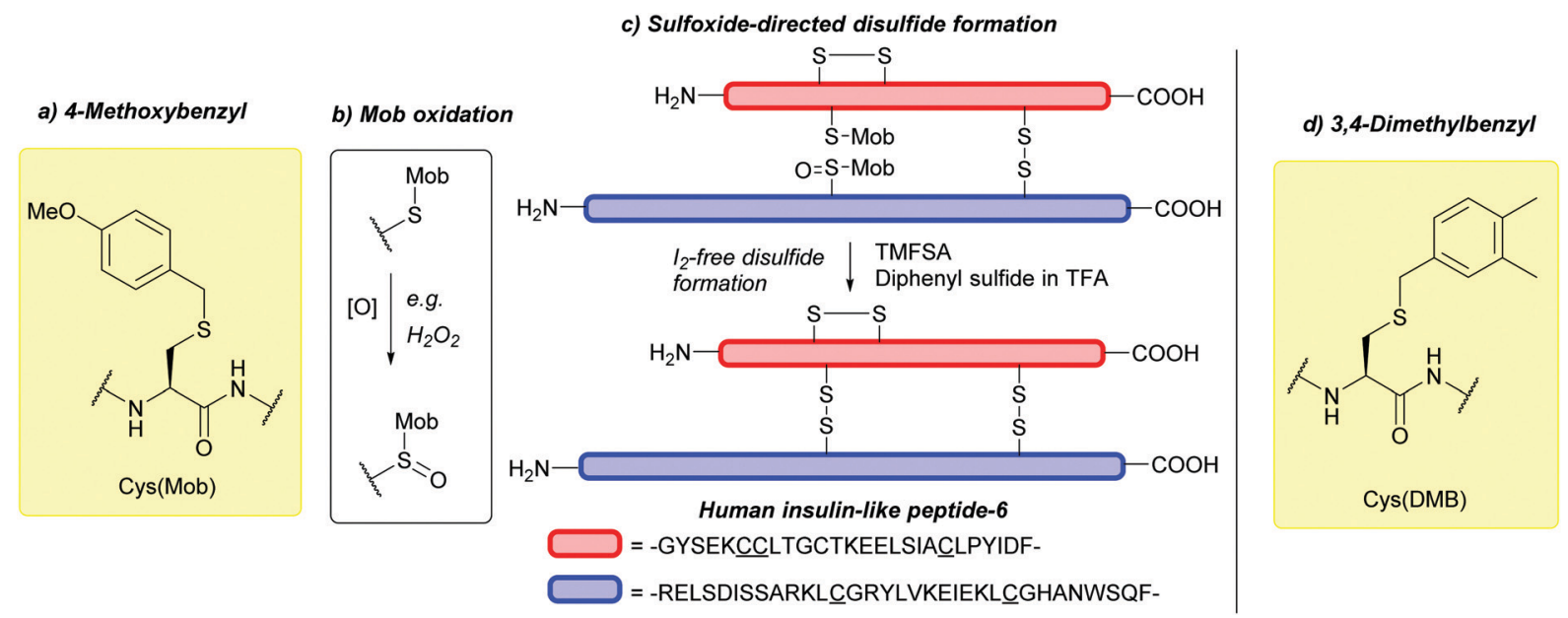

Fig. 10 (a) Cys thiol protection with the 4-methoxybenzyl (Mob) protecting group. (b) Oxidation of Cys(Mob) gives the corresponding Mob-protected sulfone, Cys(Mob(O)). (c) Synthesis of human insulin-like peptide-6 (INSL-6) using Cys(Mob) and Cys(Mob(O)) for sulfoxide-directed disulfide formation. (d) Cys thiol protection with the 3,4-dimethylbenzyl (DMB) protecting group. 
using $\mathrm{HF}\left(10 \mathrm{~min}\right.$ at $\left.0{ }^{\circ} \mathrm{C}\right)$. Its use was demonstrated by the solid-phase synthesis of the C-terminal cyclic dodecapeptide of human pituitary growth hormone, ${ }^{92}$ oxytocin derivatives, ${ }^{191}$ and melanotropin analogues. ${ }^{192}$

We note to the reader that the $\mathrm{DMB}$ protecting group is different to that of the similarly abbreviated 2,4-dimethoxybenzyl $(\mathrm{Dmb})$ group, which is used as an amide backbone protecting group to prevent peptide aggregation and off-target reactions during peptide synthesis. ${ }^{4}$

5.2.7 Methylbenzyl (Meb). The methylbenzyl (Meb, 4-MeBn, 4-MeBzl) protecting group (Fig. 11a) is broadly similar to Mob but less labile to TFA. Meb can be removed using HF-anisole $(50 \%, 1 \mathrm{~h}$, $\left.0{ }^{\circ} \mathrm{C}\right) .{ }^{91} \mathrm{Tl}(\mathrm{TFA})_{3}$ treatment of Boc-Cys(Meb)-OH will lead to cystine formation, along with minor by-products (anticipated to be sulfoxide species). ${ }^{83}$ Oxidation may also be achieved using DMSO/TFA at $45{ }^{\circ} \mathrm{C}$. Meb is orthogonal to Trt, Acm, $t$ Bu and StBu. ${ }^{74,88}$ Consequently, these protecting groups have been used together, for example, in the regioselective synthesis of human hepcidin (Fig. 11b), ${ }^{74}$ and in the synthesis of Cys-rich peptides such as protoxin I (ProTx-I), a three-disulfide-containing peptide isolated from tarantula (Thrixopelma pruriens) venom. ${ }^{193}$

5.2.8 1-Adamantyl (Ad). The 1-adamantyl (Ad, 1-Ada) group (Fig. 12a) was first introduced as a Cys protecting group in 1978,

\section{a) Methylbenzyl}

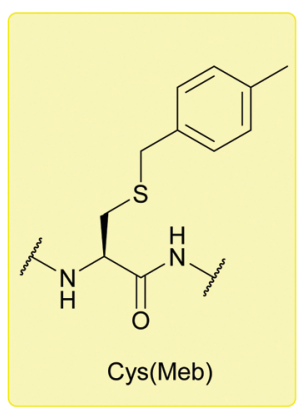

b) Regioselective disulfide formation of human hepcidin
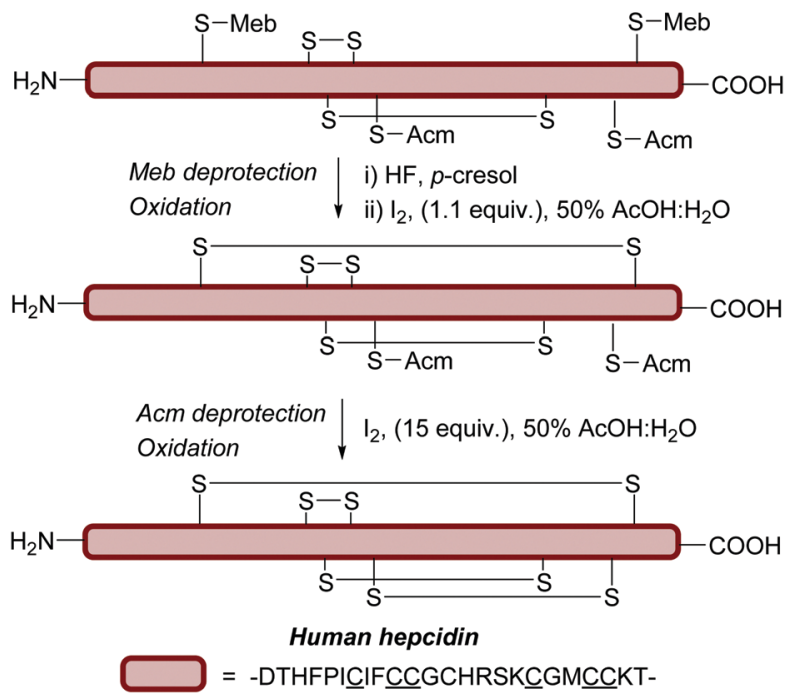

Fig. 11 (a) Cys thiol protection with the methylbenzyl (Meb) protecting group. (b) Synthesis of human hepcidin using Cys(Meb). where it was noted that it could be cleanly removed using $\mathrm{Hg}(\mathrm{OAc})_{2}$ in TFA. ${ }^{81}$ It is less labile to TFA than Mob, remaining intact under $2.5 \mathrm{~h}$ TFA treatment at $0{ }^{\circ} \mathrm{C}$. It can, however, be removed in $1 \mathrm{M}$ TFMSA-anisole/TFA in the presence of $m$-cresol, whereas application of $\mathrm{Tl}(\mathrm{TFA})_{3}$ causes disulfide formation. Ad is less susceptible to sulfoxide formation than Mob - taking $28 \mathrm{~h}$ $v s .18 \mathrm{~h}$ for complete oxidation when treated with $\mathrm{NaBO}_{3}$. This is advantageous for the synthesis of relatively large peptides. ${ }^{93}$ It is also stable to AgOTf in TFA, and is thus orthogonal to Acm, Bam and Mob. ${ }^{87}$ Cys(Ad) has previously been employed in synthesis of calcitonin-based peptides via Boc solution ${ }^{194}$ and solid phase synthesis, ${ }^{87}$ and in the synthesis of four-helix bundle proteins in Fmoc SPPS (Fig. 12b). ${ }^{195}$

a) 1-Adamantyl

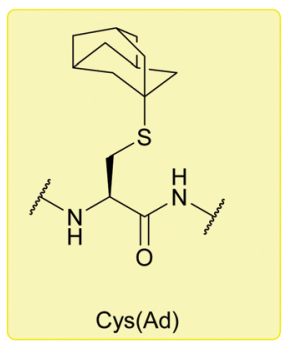

b) Four-helix bundle protein synthesis
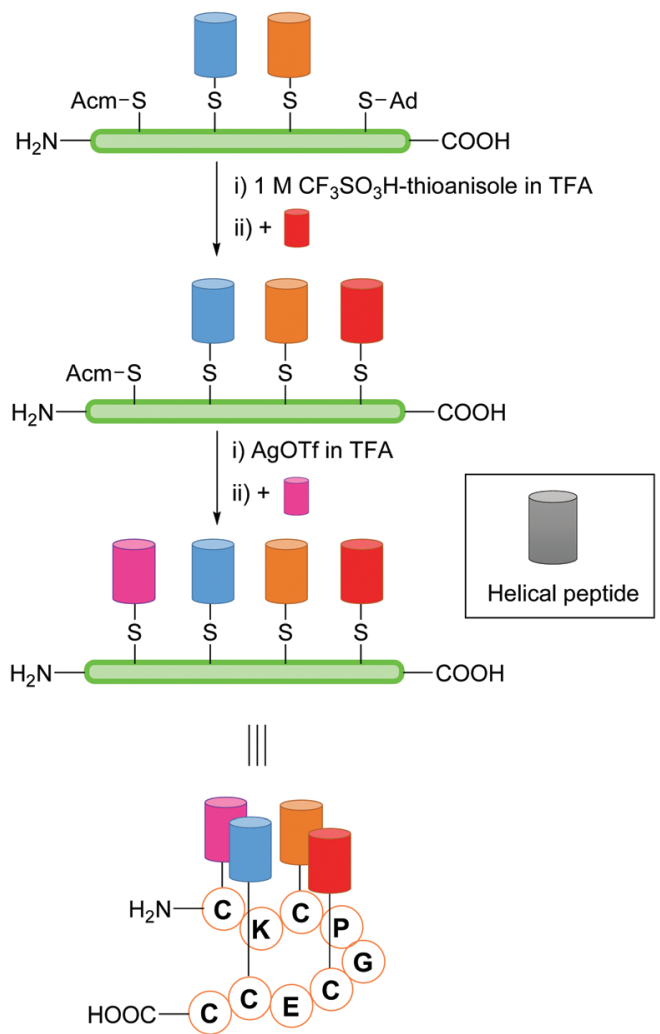

Artificial four-helix

bundle protein

Fig. 12 (a) Cys thiol protection with the 1-adamantyl (Ad) protecting group. (b) Synthesis of four-helix bundle proteins using Cys(Ad). 

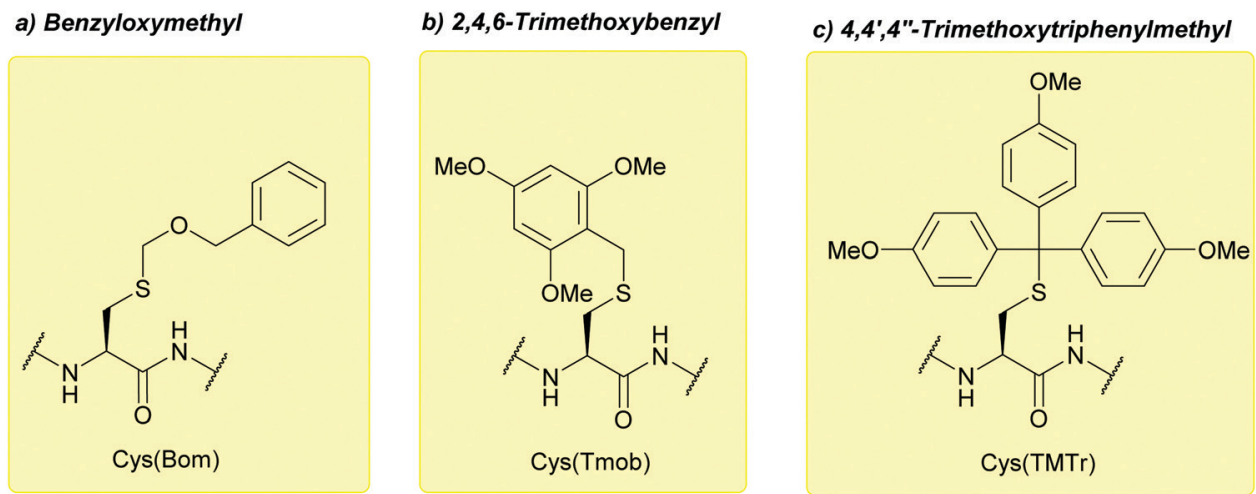

Fig. 13 (a) Cys thiol protection with the benzyloxymethyl (Bom) protecting group. (b) Cys thiol protection with the 2,4,6-trimethoxybenzyl (Tmob) protecting group. (c) Cys thiol protection with the 4,4',4"-trimethoxytriphenylmethyl (TMTr).

5.2.9 Benzyloxymethyl (Bom). Although it was already in use as a His protecting group, ${ }^{196}$ benzyloxymethyl (Bom) was introduced as a Cys protecting group (Fig. 13a) for use in Fmoc SPPS in 1989, where it was used in the synthesis of porcine brain natriuretic peptide (pBNP) ${ }^{94}$ Deprotection can be achieved using AgOTf-anisole in TFA $\left(1 \mathrm{~h}, 0{ }^{\circ} \mathrm{C}\right)$. It can also be removed using $1 \mathrm{M}$ TMSOTf-thioanisole in TFA $\left(1 \mathrm{~h}, 0{ }^{\circ} \mathrm{C}\right)$, although the former method gives a better yield of the deprotected Cys. To obtain the disulfide, $\mathrm{Tl}(\mathrm{TFA})_{3}$ may be used (but not $\left.\mathrm{I}_{2}\right)$. Bom is stable to TFA $\left(4 \mathrm{~h}, 0{ }^{\circ} \mathrm{C}\right)$, hydrazine, piperidine/ DMF and $\mathrm{NaBO}_{3}$ oxidation. ${ }^{94}$

5.2.10 2,4,6-Trimethoxybenzyl (Tmob). 2,4,6-Trimethoxybenzyl (Tmob) (Fig. 13b) was introduced in 1992 as a novel, acid-labile Cys protecting group suitable for use in Fmoc SPPS. ${ }^{96}$ Tmob can be removed by a minimum of $6 \%$ TFA in DCM, in the presence of TES or TIS $\left(5 \mathrm{~min}, 25{ }^{\circ} \mathrm{C}\right)$. Tmob can also be removed in a mixture of phenol, thioanisole and $\mathrm{H}_{2} \mathrm{O}$ ( $5 \%$ each) but, in this case, at least 30\% TFA is required. Tmob groups may be removed and the Cys thiol subsequently oxidised to the disulfide using $\mathrm{I}_{2}$ in DMF $\left(0{ }^{\circ} \mathrm{C}\right)$ or $\mathrm{Tl}(\mathrm{TFA})_{3}$ in DMF-anisole $\left(0 \quad{ }^{\circ} \mathrm{C}\right)$. Peptides synthesised using Tmob showed a marked improvement in purity when compared to identical peptides synthesised using Trt. The group is orthogonal to Acm; as such, the Tmob/Acm combination can be useful for the synthesis of two disulfide bond containing-peptides. ${ }^{95}$ However, modification of Trp residues by Tmob cations during deprotection has been reported. ${ }^{71}$

5.2.11 4, $4 \mathbf{4}^{\prime}, \mathbf{4}^{\prime \prime}$-Trimethoxytriphenylmethyl (TMTr). 4,4', $4^{\prime \prime}$ Trimethoxytriphenylmethyl (TMTr) (Fig. 13c) is removed by $1 \%$ TFA in DCM, making it more acid-labile than either Tmob or Trt. TMTr was designed to allow the selective generation of free Cys residues during Fmoc SPPS whilst the peptide is still on-resin. ${ }^{96}$

5.2.12 Pseudoprolines ( $\Psi$ Pro). Pseudoprolines ( $\Psi$ Pro) were first introduced to SPPS in 1992 as a tool for disrupting unwanted secondary structure formation on resin. ${ }^{197}$ The formation of such structures can negatively affect the solubility of a peptide in polar organic solvents and decrease coupling efficiency. By using $\Psi$ Pro to simultaneously protect the side chains and amino group of Ser, Thr or Cys, a proline-resembling oxazolidine (or thiazolidine) is formed. ${ }^{197}$ These structures are typically incorporated into peptides using dipeptide building blocks e.g. Fmoc-aaa-Cys $\left(\Psi^{\mathrm{R}, \mathrm{R}^{\prime}}\right.$ pro)-OH, where aaa = any amino acid and $\mathrm{R} / \mathrm{R}^{\prime}=$ identity of the $\mathrm{C} 2$ substituents (Fig. 14a). ${ }^{97}$ YPro imparts similar conformational properties on the peptide to proline (which can adopt a cis conformation), preventing the aggregation of hydrophobic residues by disrupting backbone hydrogen bonding. ${ }^{99,197}$ A follow up study in 1996 investigated the differing stability of $\Psi$ Pro derivatives to acid; by changing the $\mathrm{R} / \mathrm{R}^{\prime}$ groups of the $\Psi$ Pro, the lability of the protecting group to acid changes significantly. ${ }^{97}$ In the case where $\mathrm{R} / \mathrm{R}^{\prime}=\mathrm{Me}\left(\Psi^{\mathrm{Me}, \mathrm{Me}}\right.$ pro), a protected Cys can be deprotected by TFA within hours. This can be reduced to minutes if $\mathrm{R}=\mathrm{H}$ and $\mathrm{R}^{\prime}=2$,4-dimethoxyphenyl (Dmp) $\left(\Psi^{\mathrm{H}, \mathrm{Dmp}}\right.$ pro). Alternatively, when $\mathrm{R} / \mathrm{R}^{\prime}=\mathrm{H}\left(\Psi^{\mathrm{H}, \mathrm{H}}\right.$ pro, i.e., Thz $)$, the group is stable to strong acids. It should also be noted that the kinetics of deprotection appear to show some dependency on the acid and solvent system used..$^{97}$ The $\Psi$ Pro derivatives are all stable to $20 \%$ piperidine in DMF, and are thus suitable for use in Fmoc SPPS. ${ }^{97}$ As Cys $\left(\Psi^{\mathrm{H}, \mathrm{Dmp}}\right.$ pro $)$ can be readily cleaved by $90 \%$ TFA, it has been suggested to be the most appropriate $\Psi$ Pro protecting group for this strategy. The protecting group also shows stablilty to $\operatorname{Pd}(0)$, suggesting it may be orthogonal to allylbased protecting groups. ${ }^{197}$ It may also be possible exploit the differing stability of the various $\Psi$ Pro derivatives by using them together as orthogonal protecting groups. ${ }^{97}$ The acidic stability of $\mathrm{Cys}\left(\Psi^{\mathrm{Me}, \mathrm{Me}}\right.$ pro $)$ appears to be highly sequence dependant. For example, deprotection of linear 21-mer Sarafotoxin-S6b containing $\operatorname{Cys}\left(\Psi^{\mathrm{Me}, \mathrm{Me}}\right.$ pro $)$ required removal times of $32-36 \mathrm{~h},{ }^{97}$ whereas a more recent publication observed complete deprotection of $\mathrm{Cys}\left(\Psi^{\mathrm{Me}, \mathrm{Me}}\right.$ pro)-containing linear 4-10-mer peptides within 1-6 h. ${ }^{99}$ In the case of protected head-to-tail cyclic peptides, high TFA stability has been observed, with required deprotection times of 13 days reported; complete $\mathrm{Cys}\left(\Psi^{\mathrm{Me}, \mathrm{Me}}\right.$ pro $)$ deprotection can alternatively be achieved with TFMSA at $0{ }^{\circ} \mathrm{C}$ within $15 \mathrm{~min}^{.98}$ The use of $\mathrm{Cys}\left(\Psi^{\mathrm{Me}, \mathrm{Me}}\right.$ pro $)$ can enhance the rate of peptide macrocyclization, as the "kinks" induced in the peptide backbone by the $\Psi$ Pro-protected residues may bring the reacting ends closer together. This was demonstrated by the synthesis of the $\alpha$-conotoxins CnIB and A1.4 which cyclised significantly faster using $\mathrm{Cys}\left(\Psi^{\mathrm{Me}, \mathrm{Me}}\right.$ pro $)$ in place of Cys(Trt). ${ }^{99}$ In the first reported synthesis of cyclogossine B, 
a) Pseudoprolines

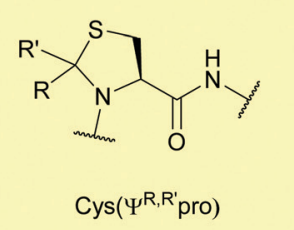

b) Synthesis of cyclogossine $B$

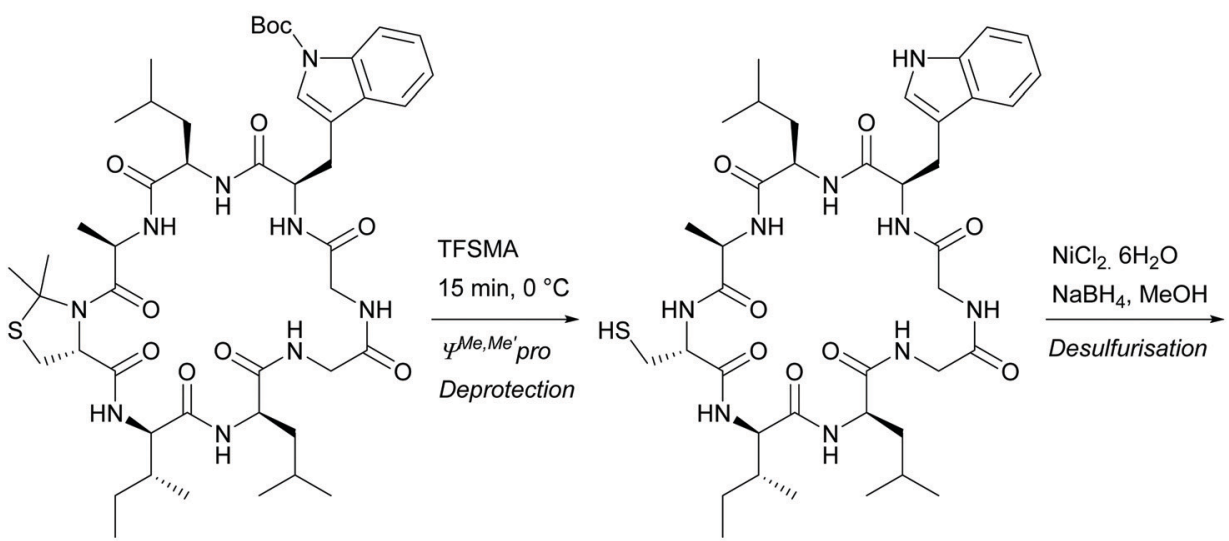

c) Synthesis of human C-type natriuretic peptide 22
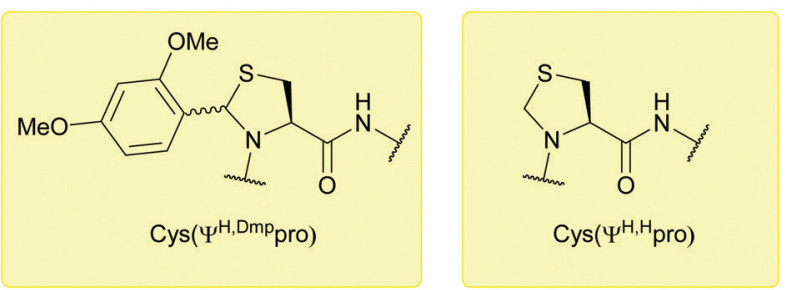<smiles>[R]OC(=O)C1CS[C@@H](c2ccc(OC)cc2OC)N1C(=O)CNC(F)F</smiles>

Fmoc-Gly-Cys $\left(\Psi^{\mathrm{H}, \mathrm{Dmp} p r o}\right)-\mathrm{OH}$

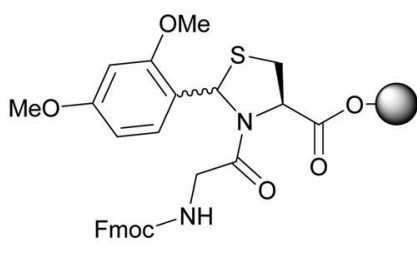

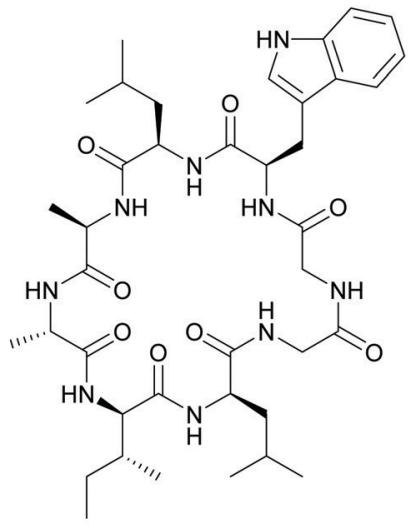

Cyclogossine B

Fig. 14 (a) Cys thiol protection with pseudoprolines bearing different substituents. (b) Synthesis of cyclogossine B using Cys( $\Psi^{\text {Me,Me }}$ pro). (c) Synthesis of human C-type natriuretic peptide 22 using a resin-bound C-terminal protected Gly-Cys $\left(\Psi^{\mathrm{H}, \mathrm{Dmp}}\right.$ pro $)$ dipeptide.

$\mathrm{Cys}\left(\Psi^{\mathrm{Me}, \mathrm{Me}}\right.$ pro $)$ was introduced in place of an Ala residue where it was used as a turn-inducer to improve cyclisation yields. Following cyclisation, a desulfurisation reaction was used to give the native Ala (Fig. 14b).$^{98}$ Additionally, replacement of Pro residues with $\mathrm{Cys}\left(\Psi^{\mathrm{Me}, \mathrm{Me}}\right.$ pro $)$ in synthetic cyclic peptide phakellistatin 19 leads to a peptide analogue with greater cytotoxicity in vitro. ${ }^{101}$ Additionally, it has recently been reported that the use of C-terminal resin-bound Cys $\left(\Psi^{\mathrm{H}, \mathrm{Dmp}}\right.$ pro) (Fig. 14c) can be used to suppress C-terminal epimerisation. ${ }^{100}$ When short peptides were treated with $20 \%$ piperidine for $24 \mathrm{~h}$ at room temperature, epimerisation levels of $<0.01 \%$ were observed. Deprotection can be achieved simultaneously with resin cleavage, using a standard cocktail of TFA/TIS/ $\mathrm{H}_{2} \mathrm{O}(95: 2.5: 2.5)$ for $1 \mathrm{~h} .{ }^{100}$

5.2.13 4-Methyltrityl (Mtt). The 4-methyltrityl (Mtt) protecting group (Fig. 15a) is a highly acid labile group that can be deprotected using $1 \%$ TFA and scavengers. ${ }^{75}$ It has been utilised for the synthesis of glutathione (GSH) and associated analogues ${ }^{198,199}$ and in the solid phase synthesis of bicyclic guanidinium oligomers. ${ }^{200}$ More recently, Cys(Mtt) has been used for the construction of peptide amphiphiles. ${ }^{201}$ In this example, dually palmitoylated peptides bearing $\mathrm{Cys}(\mathrm{Mtt})$ were first synthesised via Fmoc SPPS. Selective removal of Mtt $(2.5 \%$ TFA, $5 \%$ TIS in DCM), followed by activation with $2,2^{\prime}$-dithiobis(5nitropyridine) (DNTP) (Fig. 15b) enabled intermolecular disulfide formation with bioactive, cell penetrating peptides to construct peptide amphiphiles; these then self-assembled into the corresponding peptide amphiphile micelles. B cell lymphoma targeting aptamer (C10.36) could then be annealed to these structures to design aptamer-displaying micelles with anti-cancer properties. ${ }^{201}$ The Mtt group has also been used to protect other amino acids such as Lys. ${ }^{202}$

5.2.14 4-Methoxytrityl (Mmt). 4-Methoxyltrityl (Mmt) is a very acid-labile Cys protecting group (Fig. 16a) introduced in 1996. Mmt can be deprotected and cleaved from a resin using 1-3\% TFA in DCM-TES. Under the removal conditions stated, deprotection of Mmt is irreversible. ${ }^{71}$ This is not always the case, as some groups can re-react with the thiol following deprotection in an unwanted side reaction (e.g. Trt). ${ }^{172} \mathrm{Mmt}$ is stable to bases and very weak acids: $30 \%$ piperidine in DMF $\left(24 \mathrm{~h}, 22{ }^{\circ} \mathrm{C}\right)$ and AcOH/TFE/DCM $(1: 2: 7,30 \mathrm{~min})$. Most of its 


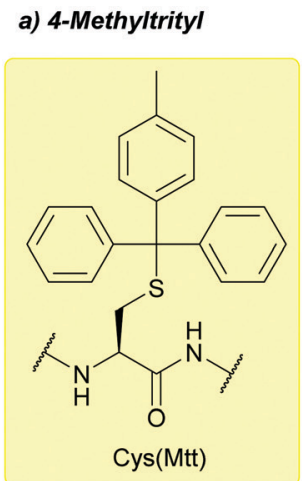

b) Mtt deprotection and thiol activation

Dually lipidated protected peptide

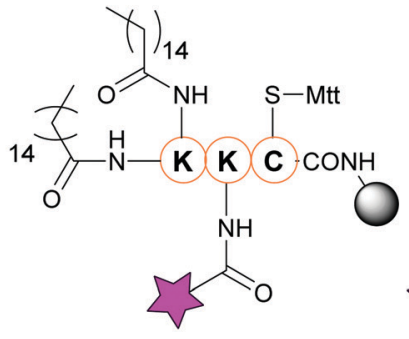

i) $2.5: 5: 92.5$ (TFA:TIS:DCM)

ii) DTNP

$=$ TAMRA dye
Dually lipidated activated peptide

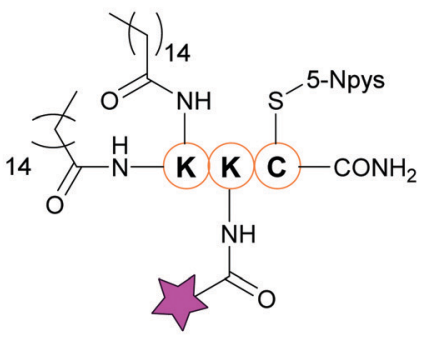

Peptide amphiphile micelle formation

Fig. 15 (a) Cys thiol protection with the 4-methyltrityl (Mtt) protecting group. (b) Deprotection of Cys(Mtt) and activation with DTNP in preparation for peptide amphiphile micelle formation

a) 4-Methoxytrityl

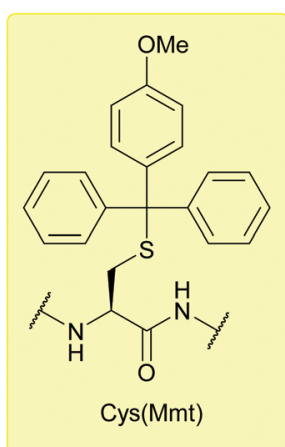

b) Partial $\alpha$-conotoxin MII synthesis

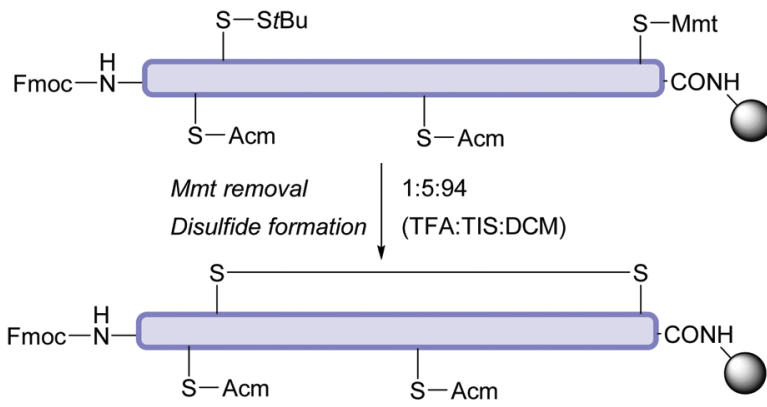

Resin bound protected $\alpha$-conotoxin MII

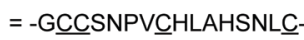

Fig. 16 (a) Cys thiol protection with the 4-methoxyltrityl (Mmt) protecting group. (b) Cys(Mmt) deprotection and subsequent disulfide formation through displacement of StBu in the synthesis of $\alpha$-conotoxin MII.

other properties, such as coupling rates, stability to piperidine, oxidative removal using $\mathrm{I}_{2}$, are identical to those of Trt. However, no modification of Trp residues is seen when using thiols or silanes as Mmt cation scavengers, which is advantageous when compared to using Tmob or Trt. $^{71}$ The Mmt group displays orthogonality to multiple Cys protecting groups, including $t \mathrm{Bu},{ }^{84}$ $\mathrm{Dpm},{ }^{75} \mathrm{oNv},{ }^{142} \mathrm{StBu},{ }^{84}$ and Acm. ${ }^{203}$ As a result, Cys(Mmt) has been frequently used in combination with other Cys protecting groups to synthesise disulfide containing peptides, such as $\alpha$-conotoxin
MII (Fig. 16b). ${ }^{203}$ In addition, as it is more acid-labile than Trt, the two groups are technically orthogonal to each other; care, however, must be taken if using Mmt and Trt together to avoid an overlap in deprotection conditions (4-5\% Trt deprotection is observed in 1\% TFA for 30 min at $22{ }^{\circ}$ C). ${ }^{71,75} \mathrm{Cys}(\mathrm{Mmt})$ has also been used in the synthesis of cyclic tumour-homing peptides, ${ }^{204}$ and conopeptides in combination with Cys(Allocam). ${ }^{205}$ The protecting group also features in the synthesis of palmitoylated peptide fragments; these peptides can be subsequently used as building blocks in Ser/Thr ligation for the chemical synthesis of complex, palmitoylated membrane proteins, including $S$-palmitoylated rabbit sarcolipin $\left(S\right.$-palm SLN), ${ }^{206} S$-palmitoylated interferon-induced transmembrane protein 3 (S-palm IFITM3), ${ }^{207}$ and $S$-palmitoylated matrix-2 ion channel from influenza A virus (S-palm M2). ${ }^{206,207}$

5.2.15 9H-Xanthen-9-yl (Xan) and 2-methoxy-9H-xanthen-9-yl (2-Moxan). The $9 H$-xanthen-9-yl (Xan) ${ }^{208}$ (Fig. 17a) and 2-methoxy$9 H$-xanthen-9-yl (2-Moxan) (Fig. 17b) groups were introduced for use in Fmoc SPPS in 1997. ${ }^{102}$ 2-Moxan is somewhat more acidlabile than Xan, but both groups are removed by $0.1 \%$ TFA in the presence of TES. Silane scavengers are highly efficient at aiding cleavage, whilst conventional aromatic scavengers, e.g. thioanisole, show no effect. Thiol scavengers, e.g. BME, may be used, but $10 \%$ TFA and a great excess of scavenger are needed. In the solid phase, more concentrated acid is required, i.e. $1 \%$ TFA for $2 \mathrm{~h}$ is sufficient. For oxidation to give a disulfide species, $\mathrm{I}_{2}$ in $\mathrm{MeOH}$

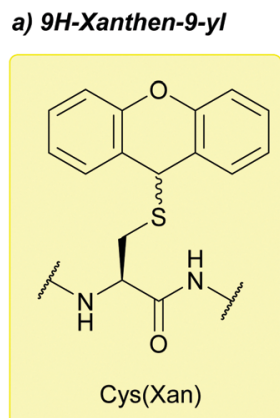

b) 2-Methoxy-9H-xanthen-9-yl

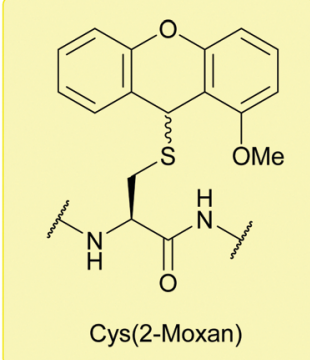

Fig. 17 (a) Cys thiol protection with the $9 H$-xanthen-9-yl (Xan) protecting group. (b) Cys thiol protection with the 2-methoxy-9H-xanthen-9-yl (2Moxan). 
or $\mathrm{Tl}(\mathrm{TFA})_{3}$ may be used. ${ }^{102}$ Both groups are stable to reagents used in Fmoc SPPS (piperidine in DMF at $25{ }^{\circ} \mathrm{C}$ for more than $24 \mathrm{~h}, 1$-hydroxybenzotriazole (HOBt) in DMF at $25{ }^{\circ} \mathrm{C}$ for $24 \mathrm{~h}$, etc.) and $\mathrm{AcOH}$ (even in the presence of silane scavengers). They can be selectively removed from acid-labile PAL (peptideamide-linker) supports, further expanding their potential use. Both groups produced sample peptides of greater purity than the equivalent peptides synthesised using Cys(Trt). These groups may thus have potential as alternatives to Trt, Tmob or Acm. ${ }^{102}$ Xan has featured in the on-resin synthesis of cyclic peptides. ${ }^{209}$

5.2.16 4,5,6-Trimethoxy-2,2-dimethyl-2,3-dihydrobenzofuran7-methyl (Tmbm), 2,2,5,7,8-pentamethylchroman-6-methyl (Pmcm) and 2,2,4,6,7-pentamethyl-2,3-dihydrobenzofuran-5-methyl (Pbfm). 4,5,6-Trimethoxy-2,2-dimethyl-2,3-dihydrobenzofuran-7-methyl (Tmbm, Fig. 18a), 2,2,5,7,8-pentamethylchroman-6-methyl (Pmcm Fig. 18b) and 2,2,4,6,7-pentamethyl-2,3-dihydrobenzofuran-5methyl (Pbfm, Pmbf, Fig. 18c) were reported as Cys protecting groups in 2010 in an attempt to find an alternative to Trt. ${ }^{103}$ Cys(Tmbm) and Cys(Pmcm) were all cleaved by TFA (although different concentrations were required). However, Pbfm was found to be a superior protecting group candidate, due to its lability and the desired solubility profile. ${ }^{103} \mathrm{Pbfm}$ shows promise as a less hydrophobic Trt replacement, with the greater solubility of Pbfm allowing for easier removal during work-up. Pbfm displays similar lability to Trt, and is removed by $1 \%$ TFA in the presence of either TES or TIS, although it should be noted that TES may not be used for Trp-containing peptides. Pbfm can also be removed oxidatively on the solid phase using $\mathrm{I}_{2}$. For oxidative removal, fluorinated acidic solvents, such as HFIP or TFE in DMF, work well, requiring only 15 min of treatment. ${ }^{103}$

5.2.17 4-Methoxybenzyloxymethyl (Mbom). 4-Methoxybenzyloxymethyl (Mbom) was introduced in 2012 as a Cys racemisationsuppressing replacement (Fig. 18d) for Trt or Acm for use in Fmoc SPPS. ${ }^{104}$ Racemisation levels during the activation and coupling steps of conventional Fmoc SPPS were found to be just $0.4 \% v s$. $8.0 \%$ for $\mathrm{Trt}^{106}$ an d $4.8 \%$ for Acm. ${ }^{104}$ Mbom can be removed using reagent $\mathrm{K}$ (a standard cleavage cocktail composed of TFA $/ \mathrm{H}_{2} \mathrm{O}$ / phenol/thioanisole/ethane-1,2-dithiol (EDT), v/v 82.5:5:5:5:2.5. Both methoxybenzyl cations and formaldehyde are generated during TFA cleavage, which can undergo side reactions with the peptide; the methoxybenzyl cations can alkylate Cys residues, whereas formaldehyde can lead to hydroxymethylation, and react with N-terminal Cys residues producing Thz-capped peptides. Such side reactions are prevented by using a thiol as a methoxybenzyl cation scavenger, and $\mathrm{MeONH}_{2} \cdot \mathrm{HCl}$ as a formaldehyde scavenger. $^{104}$

5.2.18 2,6-Dimethoxybenzyl (2,6-diMeOBn) and 4-methoxy2-methylbenzyl (4MeO-2MeBn). 2,6-Dimethoxybenzyl (2,6-diMeOBn, Fig. 18e) and 4-methoxy-2-methylbenzyl (4MeO-2MeBn, Fig. 18f) were reported by Ramos-Tomillero et al. in 2012, when searching for protecting groups that fill the gap between Trt and Mob in Fmoc SPPS, i.e. labile to TFA concentrations of $\sim 60-90 \%$. $^{75}$ Both groups fulfil this requirement and are removed using $50 \%$ TFA $\left(1 \mathrm{~h}, 25^{\circ} \mathrm{C}\right)$. Despite this, Dpm was recommended for use instead, due to its similar properties and easier accessibility. ${ }^{105}$
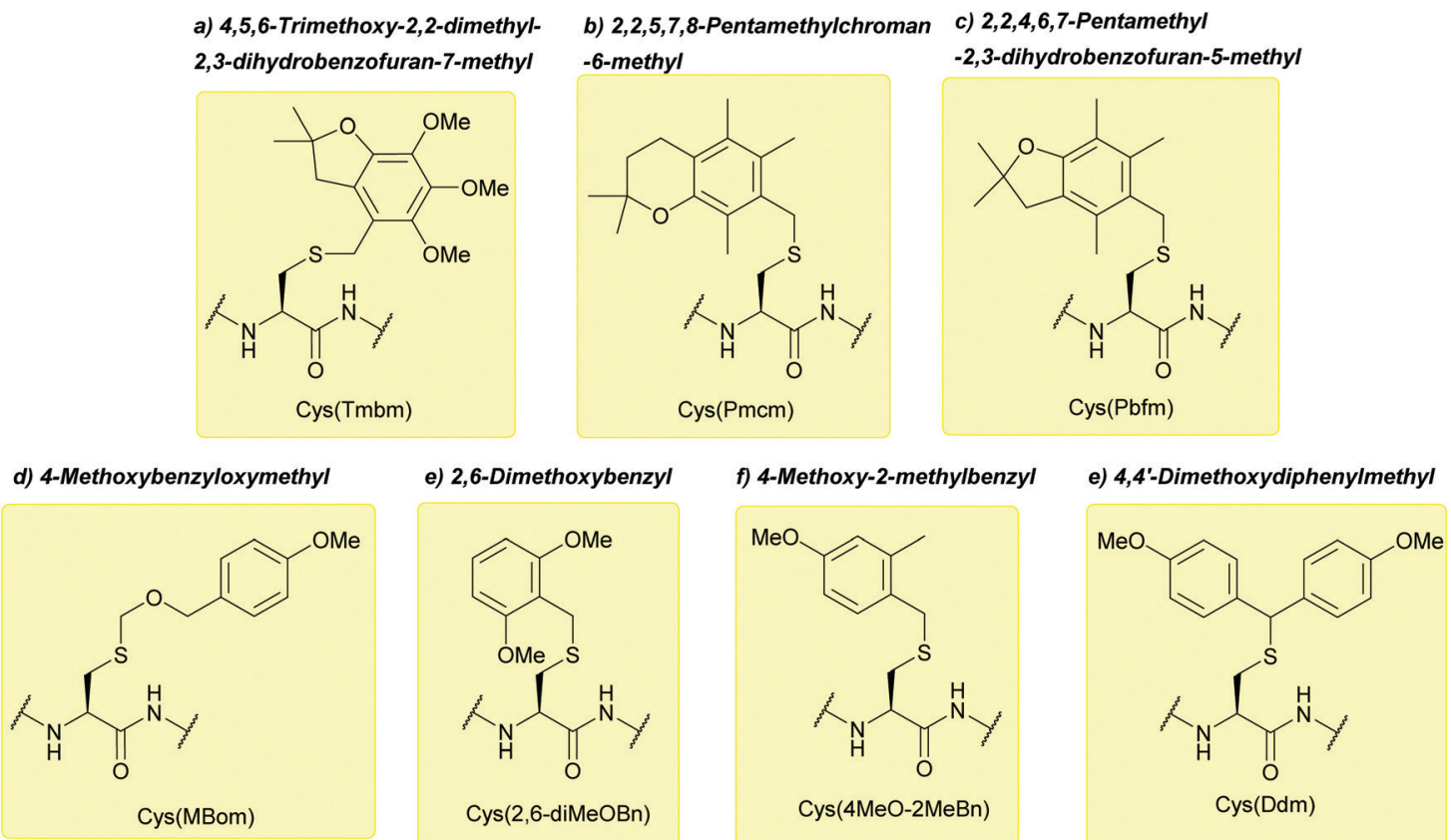

Fig. 18 (a) Cys thiol protection with the 4,5,6-trimethoxy-2,2-dimethyl-2,3-dihydrobenzofuran-7-methyl (Tmbm) protecting group. (b) Cys thiol protection with the 2,2,5,7,8-pentamethylchroman-6-methyl (Pmcm) protecting group. (c) Cys thiol protection with the 2,2,4,6,7-pentamethyl-2,3dihydrobenzofuran-5-methyl (Pbfm) protecting group. (d) Cys thiol protection with the 4-methoxybenzyloxymethyl (Mbom). (e) Cys thiol protection with the 2,6-dimethoxybenzyl (2,6-diMeOBn) protecting group. (f) Cys thiol protection with the 4-methoxy-2-methylbenzyl (4MeO-2MeBn). (g) Cys thiol protection with the $4,4^{\prime}$-dimethoxydiphenylmethyl (Ddm) protecting group. 
a) 2-Hydroxy-4-methoxybenzyl

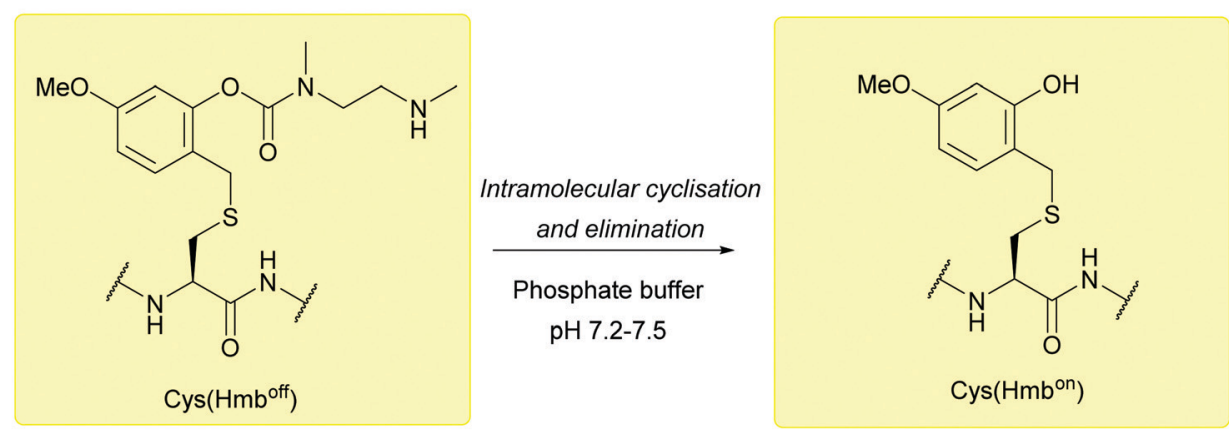

b) Conversion of $\mathrm{Hmb}^{\text {off }}$ to $\mathrm{Hmb}^{\text {on }}$

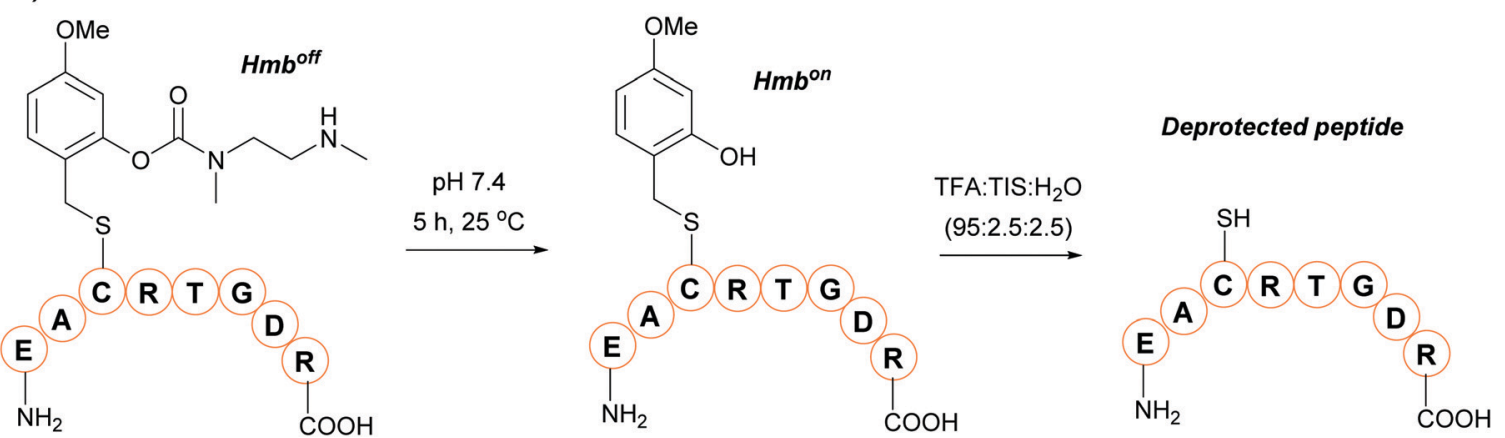

Fig. 19 (a) Cys thiol protection with the $\mathrm{Hmb}^{\text {off/on }}$ protecting group. (b) Conversion of $\mathrm{Cys}\left(\mathrm{Hmb}^{\text {off }}\right)$ to $\mathrm{Cys}\left(\mathrm{Hmb}^{\text {on }}\right)$ and subsequent deprotection of a model peptide.

5.2.19 4,4'-Dimethoxydiphenylmethyl (Ddm). 4,4'-Dimethoxydiphenylmethyl (Ddm, 4,4'-diMeODpm, Fig. 18g) was introduced by Ramos-Tomillero et al. and can be removed in 10\% TFA ( 5 min, $\left.25{ }^{\circ} \mathrm{C}\right) .{ }^{105}$ It is recommended as a racemisation-suppressing replacement for Trt, displaying lower rates of Cys racemisation that Trt or Dpm (0.8\% vs. $8.0 \%$ vs. $1.2 \%$, (respectively)) when used for conventional Fmoc SPPS. ${ }^{106}$

5.2.20 $\mathrm{Hmb}^{\text {off/on }}$. Hmb ${ }^{\text {off/on }}$, a 2-hydroxy-4-methoxy benzyl derivative, is a novel protecting group with switchable activity (Fig. 19a). Hmb ${ }^{\text {off }}$ is fully compatible with Fmoc SPPS. However, when treated with aqueous buffer at $\mathrm{pH}$ 7.2-7.5 the group is selectively converted to the $\mathrm{Hmb}^{\text {on }}$ form. The $\mathrm{Hmb}^{\text {on }}$ form is fully compatible with standard NCL protocols, including the post-ligation desulfurisation reaction and any HPLC handling steps but can be removed by TFA cocktails (Fig. 19b, 95\% TFA, 2 h, $25{ }^{\circ} \mathrm{C}$ - conditions under which $\mathrm{Hmb}^{\text {off }}$ is stable). To demonstrate its use in SPPS, a segment of erythropoietin (EPO) was synthesised

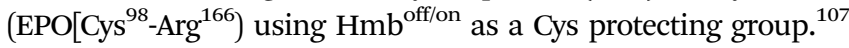

\subsection{Oxidatively-labile protecting groups}

5.3.1 Acetamidomethyl (Acm). Acetamidomethyl (Acm, Fig. 20a) was first described by Veber et al. in $1968 .{ }^{108}$ This novel protecting group was noted to be stable to acidic conditions (TFA, $\mathrm{HBr} / \mathrm{AcOH}$ and $\mathrm{HCl} / \mathrm{EtOH}$ (all at $25^{\circ} \mathrm{C}$ ), as well as anhydrous $\mathrm{HF}$ at $0{ }^{\circ} \mathrm{C}$ ). Acm was also noted to be stable to alkaline aqueous solutions at $25{ }^{\circ} \mathrm{C}$ but, crucially, could be removed by $\mathrm{Hg}(\mathrm{OAc})_{2} \cdot{ }^{108,110}$ Follow up literature reports further established that Acm shows stablilty to commonly used peptide synthesis protocols, can be removed under relatively mild conditions and displays no major racemisation problems. ${ }^{110} \mathrm{Hg}$ (II) is not the only heavy metal that can remove Acm-AgOTf in TFA (followed by DTT to obtain the free thiol) will also work, ${ }^{87}$ as will $\mathrm{AgBF}_{4}$ in TFA-thioanisole (also followed by DTT treatment). ${ }^{121}$ Alternative acidic conditions can also be used: $6 \mathrm{M} \mathrm{HCl}$ at $110{ }^{\circ} \mathrm{C}$ for $20 \mathrm{~h}^{110}$ or $97.5 \%$ TFA-thioanisole with an electrophilic disulfide (DTNP or DTP), with an excess of DTNP required (15 eq. for $\sim 90 \%$ deprotection). ${ }^{90}$ Partial Acm deprotection (70\%) of a Cys(Acm)containing peptide in $98 \%$ TFA-TIS has also been observed after $12 \mathrm{~h}$ at $37{ }^{\circ} \mathrm{C}$, with $35 \%$ of the deprotected peptide in the disulfide form and $35 \%$ present as the free thiol. Replacing TIS with anisole or phenol resulted in similar levels of deprotection, whilst $80-90 \%$ deprotection was observed when using thioanisole. TES scavenger proved more effective at promoting Acm removal (TES can however reduce the indole ring of Trp). It should be noted here that whilst thioanisole and TES promote disulfide formation, as observed with TIS, anisole and phenol do not. ${ }^{86}$ For Acm removal and subsequent oxidation to yield disulfides, $\mathrm{I}_{2}$, $\mathrm{Tl}(\mathrm{TFA})_{3},{ }^{111}$ or silyl chloride-sulfoxide in TFA may be used. ${ }^{82}$ Acm is stable to TCEP and is thus orthogonal to SiPr. ${ }^{114}$ The Acm protecting group also displays orthogonality to a large number of other Cys protecting groups; these include Trt, $t \mathrm{Bu}$, Meb, ${ }^{74}$ Ad, ${ }^{87} \mathrm{Hqm} / \mathrm{Hgm},{ }^{128}$ Msbh, ${ }^{166} \mathrm{Tmob}, \mathrm{Mmt},{ }^{203}$ and Dnpe. ${ }^{124}$ As a result, Acm is widely used as a Cys protecting groups, as shown in the synthesis of proteins including chemokine CCL27 (using $\mathrm{AgNO}_{3}$ for Cys(Acm) deprotection), ${ }^{51}$ EPO (using AgOAc for Cys(Acm) deprotection), ${ }^{210}$ and HIV-1 Rev (Fig. 20b, using $\mathrm{Hg}(\mathrm{OAc})_{2}$ for Cys(Acm deprotection). ${ }^{210} \mathrm{How}^{-}$ ever, the methods described thus far to deprotect Cys(Acm) 


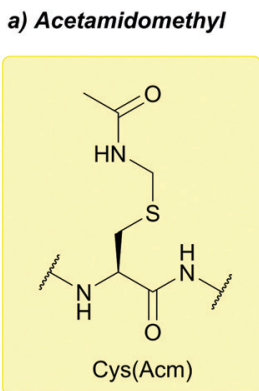

b) Synthesis of HIV-1 Rev

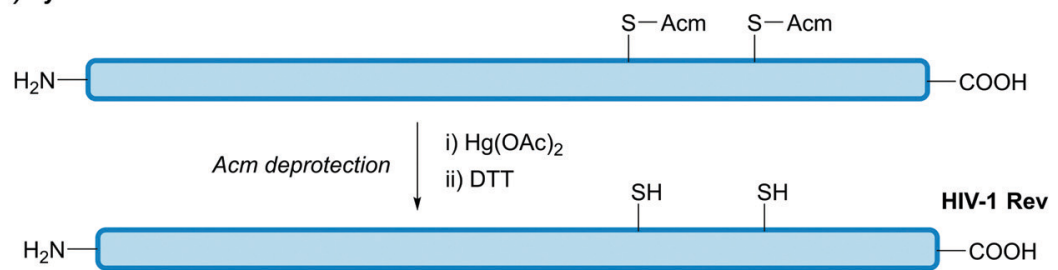

MAGTSGDSDEELIRTVRLIKLLYQSNPPPNPEGTRQARRNRRRRQRERQRQIHSISERI LGTYLGRSAEPVPLQLPPLERLTLDCNNEDCGGTSGTQGVGSPQILVESPTVLESGTKE

c) Synthesis of thioredoxin-1
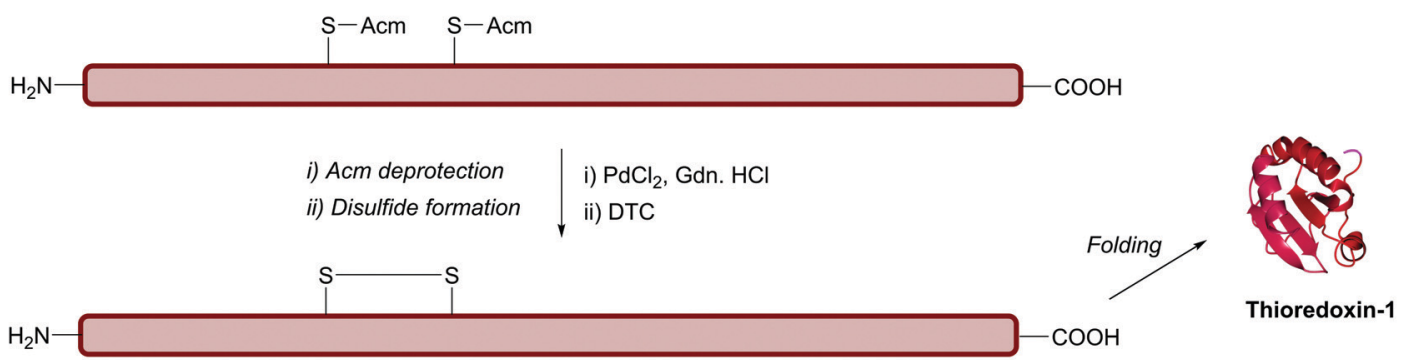

$=\begin{aligned} & -{ }^{n} \text { LSDKIIHLTDDSFDTDVLKADGAILVDFWAEW } \underline{\text { CGPCEKMIAPILDEIADEYQGKLT }} \\ & \text { VAKLNIDQNPGTAPKYGIRGIPTLLLFKNGEVAATKVGALSKGQLKEFLDANLA- }\end{aligned}$

d) Synthesis of Ecballium elaterium trypsin inhibitor II

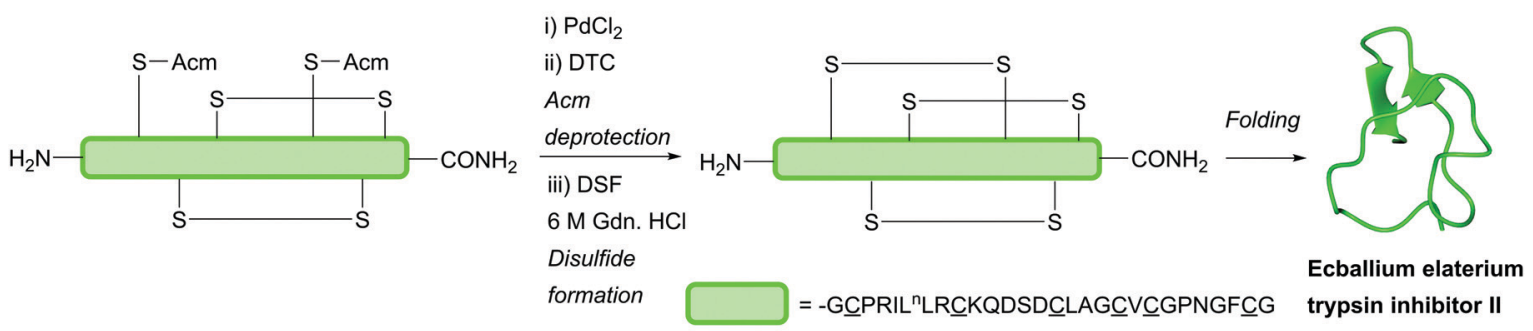

e) Synthesis of apamin
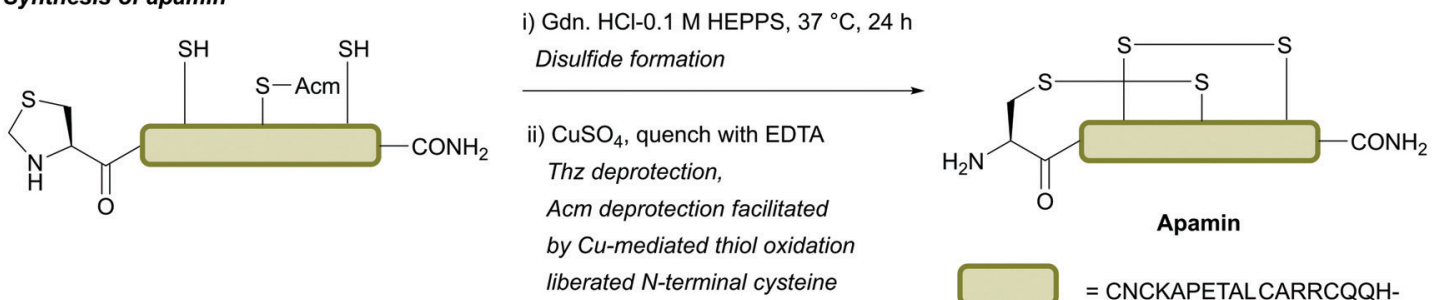

by $\mathrm{Cu}$-mediated thiol oxidation
liberated $\mathrm{N}$-terminal cysteine

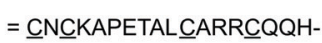

Fig. 20 (a) Cys thiol protection with the acetamidomethyl (Acm) protecting group. (b) Synthesis of HIV-1 Rev using Cys(Acm) and Hg-mediated deprotection. (c) Synthesis of thioredoxin-1 using Cys(Acm) and Pd-mediated deprotection. DTC is used for Pd quenching. (d) Synthesis of Ecballium elaterium trypsin inhibitor II (EETI-II) using Cys(Acm) and Pd-mediated deprotection. DSF is used for disulfide formation post-Acm removal. (e) Synthesis of apamin using $\mathrm{Cys}(\mathrm{Acm})$ and a $\mathrm{Cu}$-mediated deprotection process.

have been reported to suffer from several issues over the years. The heavy metals used to remove Acm are toxic and harmful to the environment, and $S$ to $O(\mathrm{Ser}, \mathrm{Thr}) \mathrm{Acm}$ shifts can occur upon deprotection in peptides with high Ser or Thr content. This can be avoided by using glycerol (with a molar ratio of glycerol:peptide, 5600:1) as a scavenger with $\mathrm{Hg}$ (II) or $\mathrm{Tl}(\mathrm{III})$ deprotection methods. ${ }^{211} \mathrm{I}_{2}$ can cause back-alkylation, resulting in the formation of residual products, which can be difficult to remove. ${ }^{127}$ Other potential side-reactions include the iodination of Trp and Tyr residues ${ }^{128}$ or over-oxidation to the sulfonic acid. ${ }^{109}$ Although mostly acid-stable, premature cleavage of Acm occasionally occurs in mildly acidic conditions in the presence of free thiol and thioanisole scavengers. Irreversible alkylation of Tyr residues with Acm groups can then occur. This reaction appears to be suppressed with scavengers (e.g. phenol) at comparatively high peptide dilution and may be sequence-specific. ${ }^{212}$ The preparation of Acm can also be problematic, as thiazolidine-2-carboxylic acid can be formed as a 
byproduct. Activation using DCC/HOBt can also result in side reactions. The Bam and Tacm protecting groups have been reported in an attempt to address these issues. ${ }^{120}$ Due to its partial instability to $\mathrm{HF}$, Acm is not particularly suitable for use in Boc SPPS. ${ }^{111}$ In addition, Acm reacts with strong electrophiles, such as the $t \mathrm{Bu}$ cation (which is generated during Boc removal), causing $S$-alkylation - although this reaction can be suppressed by the addition of dimethyl sulfide. ${ }^{110}$

More recently, it has been reported that Acm can be removed under significantly milder conditions using transition metal catalysts, such as Pd(II) complexes. ${ }^{109,112}$ For example, the synthesis of the two-Cys containing ubiquitin-like protein 5 (UBL5) could be achieved in a one-pot manner, with deprotection of Cys(Acm) in the final step performed using 30 equiv. $\mathrm{PdCl}_{2}$ within $15 \mathrm{~min}$ (followed by DTT addition). ${ }^{112}$ Treatment of Cys(Acm)-containing peptides with $\mathrm{PdCl}_{2}$ in $\mathrm{H}_{2} \mathrm{O}$ or guanidinium chloride $(\mathrm{Gdn} \cdot \mathrm{HCl}$ ) pH 7 (30 min, $37^{\circ} \mathrm{C}$ ) followed by DTT addition led to total deprotection to yield the free thiol-containing peptide. Alternatively, using 100 equiv. of diethyldithiocarbamate (DTC) after Pd-mediated deprotection of multi-Cys(Acm)-containing peptides allows for oxidation to form disulfide bonds. Total synthesis of E. Coli thioredoxin (Trx-1) in a one pot manner has been reported using this strategy. First NCL was performed between an N-terminal fragment (bearing two Cys(Acm) residues and a C-terminal thioester, $\operatorname{Trx}(1-56)$ ) and a C-terminal fragment (bearing an $N$-terminal Cys (Trx58-109)). This was then followed by desulfurisation, and finally sequential Acm deprotection and disulfide formation using $\mathrm{PdCl}_{2}$ and DTC respectively to yield the target protein (Fig. 20c). ${ }^{109}$ Moreover, whilst Acm and Thz are both labile to $\mathrm{Pd}(\mathrm{II})$ complexes, Acm is stable to the lower concentrations of $[\mathrm{Pd}(\text { allyl }) \mathrm{Cl}]_{2}$ compared to Thz, giving the two groups some orthogonality to one another. Following Thz deprotection, Acm can be deprotected within $5 \mathrm{~h}$ following the addition of an extra 10 equiv. of $[\mathrm{Pd}(\text { allyl }) \mathrm{Cl}]_{2}$. Additionally, Acm is orthogonal to $t \mathrm{Bu}$ under these conditions. ${ }^{85} \mathrm{Acm}$ is also stable to $\mathrm{Pd}(0)$-mediated removal of $\mathrm{N}$-terminal Alloc-protected $\alpha$-amino groups. This, in tandem with a $\beta$-thiolactone-mediated NCL procedure, ${ }^{213,214}$ was demonstrated in the full chemical synthesis of histone $\mathrm{H} 3$ bearing an $\mathrm{N} \varepsilon$ trimethylated Lys residue ([Lys(Me3)9]H3.1). ${ }^{113}$ The use of $\mathrm{PdCl}_{2}$ for Cys(Acm) deprotection, with DTC for Pd quenching and disulfiram (DSF) for disulfide formation, has very recently been described in combination with photolabile Cys protecting groups for rapid deprotection/disulfide formation in multiple disulfiderich peptides, including Linaclotide and Ecballium elaterium trypsin inhibitor II (EETI-II, Fig. 20d). ${ }^{215}$

In addition to $\mathrm{Pd}(\mathrm{II})$ chemistry, deprotection of $\mathrm{Cys}(\mathrm{Acm})$ using $\mathrm{CuSO}_{4}$ has been reported. ${ }^{73}$ Initially, it was noted that $\mathrm{CuSO}_{4}$-mediated deprotection of Cys(Thz) would also lead to deprotection of Cys(Acm) in the same peptide scaffold if ascorbate was not added, but would leave Acm intact if ascorbate was added. In model peptides, $\mathrm{CuSO}_{4}$ would only deprotect Cys(Acm) if the peptide contained an N-terminal Cys that was either unprotected or protected with Thz (which could undergo $\mathrm{CuSO}_{4}$-mediated deprotection to reveal an N-terminal Cys). This instance of double deprotection has been hypothesised to result from $\mathrm{Cu}$-mediated oxidation of the deprotected/unprotected $\mathrm{N}$-terminal thiol to sulfenic acid, which then undergoes nucleophilic attack from the Cys(Acm) sulfur, leading to disulfide formation and loss of Acm. Excess $\mathrm{Cu}$ can then be quenched with DTT (leading to the free thiol-containing peptide) or ethylenediaminetetraacetic acid (EDTA, leading to the disulfide containing peptide). This protocol was demonstrated in the synthesis of the two-disulfide containing peptide apamin. Following formation of the first disulfide bond via Cys(Trt) deprotection and air oxidation, sequential deprotection of Cys(Thz) and Cys(Acm) with $\mathrm{CuSO}_{4}$ (100 mM, $6 \mathrm{M}$ Gdn.HCl, 0.1 M HEPPS) followed by quenching with EDTA (100 mM), could be used to engineer the second disulfide bond in a regioselective manner (Fig. 20e). Oxidation by-products were formed, however, if the order of disulfide bond formation in this procedure was reversed (with Cys(Mob) used as opposed to Cys(Trt)). In the case of peptides which lack an unprotected $N$-terminal Cys, $\mathrm{Cys}(\mathrm{Acm})$ can be deprotected by addition of $\mathrm{CuSO}_{4}$ in the presence of an equimolar amount of aminothiol additive, such as cysteamine. ${ }^{73}$

5.3.2 5-Dibenzosuberyl (Dbs). The 10,11-dihydro-5 $H$-dibenzo$[a, d]$ cyclohepten-5-yl or 5-dibenzosuberyl (Dbs, Sub) protecting group (Fig. 21a) was proposed in $1976 .{ }^{115}$ The group was noted to be removed reductively with $\mathrm{Hg}(\mathrm{OAc})_{2}$ or oxidatively with $\mathrm{I}_{2}$ in $\mathrm{MeOH}$ or AcOH. ${ }^{115}$ Dbs-Protected Cys may also be cleaved using $\mathrm{Tl}(\mathrm{TFA})_{3}$ to yield cystine. ${ }^{83,216}$ Dbs was observed to have "sufficient" stability to TFA but was partially removed by $\mathrm{HBr} / \mathrm{AcOH} .{ }^{115}$ Preliminary work has shown that Dbs may be a
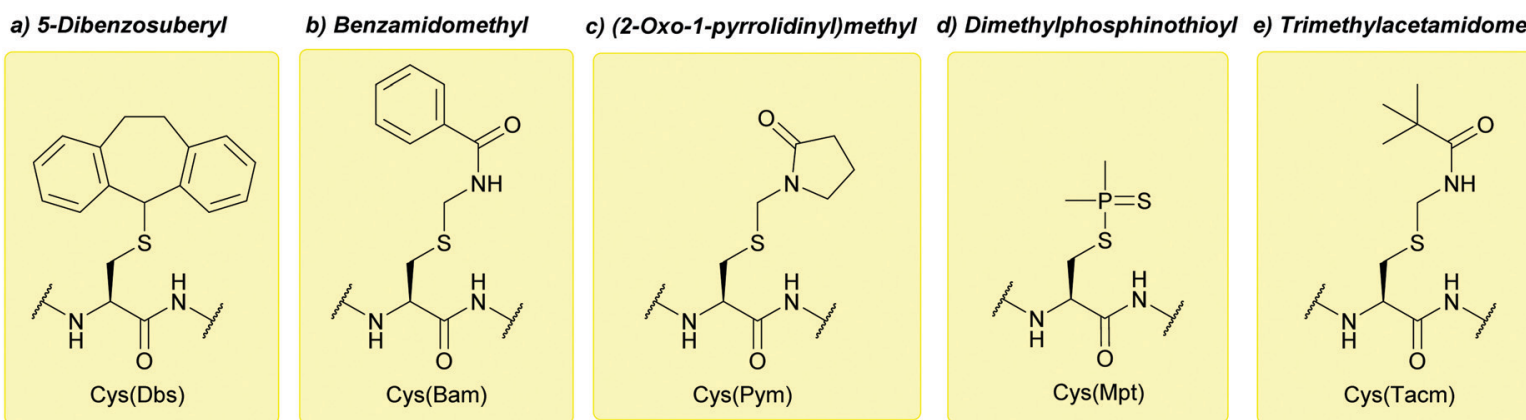

Fig. 21 (a) Cys thiol protection with the 5-dibenzosuberyl (Dbs) protecting group. (b) Cys thiol protection with the benzamidomethyl (Bam) protecting group. (c) Cys thiol protection with the (2-oxo-1-pyrrolidinyl)methyl (Pym) protecting group. (d) Cys thiol protection with the dimethylphosphinothioyl (Mpt) protecting group. (e) Cys thiol protection with the trimethylacetamidomethyl (Tacm) protecting group. 
promising protecting group for the guanidino function of $\mathrm{Arg},{ }^{217}$ however, it is yet to find widespread use as a protecting group. ${ }^{4}$

5.3.3 Benzamidomethyl (Bam). The benzamidomethyl (Bam) protecting group (Fig. 21b) was proposed in 1978 as an alternative to Acm which could still be removed using $\mathrm{Hg}(\mathrm{OAc})_{2}(1 \mathrm{~h}, \mathrm{RT}){ }^{116}$ Bam can also be removed oxidatively by AgOTf in TFA-anisole $(1 \mathrm{~h}$, $\left.0{ }^{\circ} \mathrm{C}\right)^{87}$ as well as by methyltrichlorosilane/tetrachlorosilane in the presence of diphenyl sulfoxide (a "silyl chloride-sulfoxide system") in TFA. ${ }^{82}$ DTT may then be added to obtain the free thiol. ${ }^{87}$ Compared to Acm and when no $\mathrm{N}$-terminal protecting was present, preparation of Cys(Bam) was not accompanied by the formation of thiazolidine-2-carboxylic acid and side reactions were not observed during DCC/HOBt activation. ${ }^{120}$ Bam has been shown to be stable to many commonly used reagents in peptide synthesis: $1 \mathrm{M} \mathrm{NaOH}$, $1 \mathrm{M} \mathrm{HCl}$, and TFA (all at $25{ }^{\circ} \mathrm{C}$ ), as well as $90 \% \mathrm{Zn} / \mathrm{AcOH}$ at $0{ }^{\circ} \mathrm{C}$. The group is however unstable to very strong acids, such as $6 \mathrm{M}$ $\mathrm{HCl}\left(24 \mathrm{~h}, 110{ }^{\circ} \mathrm{C}\right),{ }^{116}$ as well as HF cleavage and towards alkaline conditions. $^{120}$

5.3.4 (2-Oxo-1-pyrrolidinyl)methyl (Pym). The (2-oxo-1-pyrrolidinyl)methyl (Pym) functionality has been reported as a somewhat obscure Cys protecting group (Fig. 21c). ${ }^{117}$ Cys(Pym) shows instability when treated with $\mathrm{HF}$, and is susceptible to oxidation with $\mathrm{NaBO}_{3}$ to give the corresponding Cys sulfoxide species similar to Cys(Acm) and Cys(Bam). It has been noted to show stability towards $0.05 \mathrm{M} \mathrm{NaOH}$ or treatment with hydrazine. Complications arising from use of DCC-HOBt coupling were not observed when using Boc-Cys(Pym)-OH. ${ }^{117}$ It has been reported as a protecting group for the synthesis of hexapeptides, although its success in giving pure peptides during isolation was rather limited; this was rationalised by the Cys(Pym) group displaying increased solubility in solvents such as $\mathrm{H}_{2} \mathrm{O}$, acetone, and $\mathrm{Et}_{2} \mathrm{O}{ }^{218}$

5.3.5 Dimethylphosphinothioyl (Mpt). Initially reported as an N-terminal $\alpha$-amino protecting group, ${ }^{219}$ the dimethylphosphinothioyl (Mpt) protecting group was first investigated as a Cys protecting group in 1983 (Fig. 21d). ${ }^{118} \mathrm{Cys}(\mathrm{Mpt})$ proved stable at room temperature to $2 \mathrm{M} \mathrm{HCl}$ in solvents such as EtOAc and $\mathrm{AcOH}$, and to TFA. Partial cleavage was observed when using 25\% $\mathrm{HBr} / \mathrm{AcOH}$, and dehydroalanine formation was noted upon treating $\mathrm{Cys}(\mathrm{Mpt})$-containing peptides with 1,8-diazabicyclo[5.4.0] undec-7-ene (DBU). The Mpt group can be cleaved with $\mathrm{AgNO}_{3}$ or $\mathrm{Hg}(\mathrm{OAc})_{2}$ (1-4 equiv. 20-60 min), and has been used in the Boc solution phase synthesis of glutathione. ${ }^{118}$ Alternatively, deprotection can also be achieved using tetrabutylammonium fluoride (TBAF)/THF to give the free thiol, or TBAF/DMF to yield the corresponding disulfide. If Mpt deprotection with TBAF is carried out in DCM, however, methylenedithioether formation between two Cys thiols occurs; this was subsequently demonstrated in the Boc solution phase synthesis of a cyclic enkephalin analogue. ${ }^{119}$

5.3.6 Trimethylacetamidomethyl (Tacm). The trimethylacetamidomethyl (Tacm) group (Fig. 21e) was proposed in 1989 as an alternative to Bam. ${ }^{120}$ Similar to Bam, preparation and activation when using Tacm are not accompanied by side reactions; in contrast, however, Tacm is also stable to acidic conditions (HF, $1 \mathrm{~h}, 0{ }^{\circ} \mathrm{C}$, or TFMSA-thioanisole in TFA, $2 \mathrm{~h}$, $\left.0{ }^{\circ} \mathrm{C}\right)$ and a variety of basic conditions $(0.05 \mathrm{M} \mathrm{NaOH}$ in $\mathrm{MeOH}$, $1 \mathrm{~h}, 0{ }^{\circ} \mathrm{C}$ or hydrazine in $\left.\mathrm{MeOH}, 24 \mathrm{~h}, \mathrm{RT}\right)$. Tacm is also stable to $90 \% \mathrm{Zn} / \mathrm{AcOH}\left(1 \mathrm{~h}, 25^{\circ} \mathrm{C}\right)$, and displays a higher resistance to air oxidation than Acm or Bam. Partial cleavage is observed, however, when treated with $25 \% \mathrm{HBr} / \mathrm{AcOH} .{ }^{117}$ Tacm can be removed using $\mathrm{Hg}(\mathrm{OAc})_{2}$, or $\mathrm{I}_{2} / \mathrm{EtOH}$ in $\mathrm{AcOH}$ (aq.) for oxidation to the disulfide. ${ }^{120}$ Much like Acm, Tacm is also cleaved by $\mathrm{AgBF}_{4}$ in TFA-thioanisole (followed by DTT treatment) ${ }^{121}$ and silyl chloridesulfoxide in TFA, the latter of which gives the disulfide. ${ }^{82}$

Care must be taken when using $\mathrm{I}_{2}$ in $\mathrm{AcOH}$, as partial oxidation of methionine (Met) residues to methionine sulfoxide (Met(O)) can occur. Modification of Trp residues has also been observed. ${ }^{121}$ Despite its higher resistance to acid than Bam, Tacm can still show instability in HF and TFA/heat. It is therefore not particularly suitable for Boc SPPS, ${ }^{111}$ although it has been used successfully in Boc solution phase synthesis of pBNP using this strategy. ${ }^{120}$ Furthermore, Cys(Tacm) has been used in Fmoc SPPS to synthesise oxytocin in combination with the silyl chloride-sulfoxide system. ${ }^{20}$

\subsection{Base-labile protecting groups}

5.4.1 9-Fluorenylmethyl (Fm). The 9-fluorenylmethyl (Fm) group is a base-labile Cys protecting group (Fig. 22a) first described in $1982^{122}$ and introduced to SPPS in $1986 .{ }^{123} \mathrm{Fm}$ can be removed by $\mathrm{NH}_{3}$ in $\mathrm{MeOH}^{122}$ or $50 \%$ piperidine in DMF $(2 \mathrm{~h}, \mathrm{RT}) .{ }^{123} \mathrm{Fm}$ displays favourable properties for Boc SPPS; it is stable to TFA, TFMSA/TFA, boiling $\mathrm{HCl}\left(\text { at } 110{ }^{\circ} \mathrm{C}\right)^{122}$ as well as HF-anisole
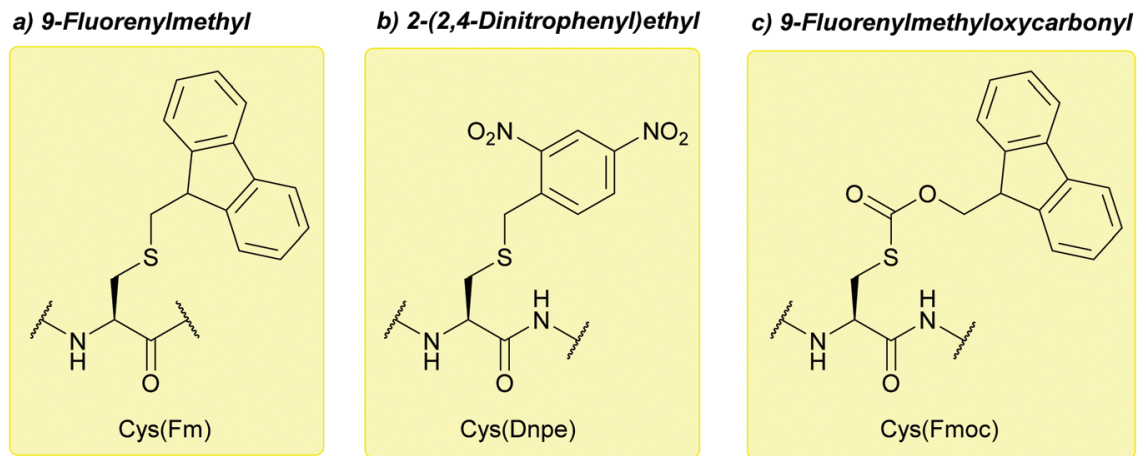

Fig. 22 (a) Cys thiol protection with the 9-fluorenylmethyl (Fm) protecting group. (b) Cys thiol protection with the 2-(2,4-dinitrophenyl)ethyl (Dnpe). (c) Cys thiol protection with the 9-fluorenylmethyloxycarbonyl (Fmoc) protecting groups. 
$\left(95: 5,1 \mathrm{~h}, 0{ }^{\circ} \mathrm{C}\right)$. It is also stable to $0.1 \mathrm{M} \mathrm{I}_{2}$ in $\mathrm{DMF},{ }^{123}$ and catalytic hydrogenation. ${ }^{122}$ As with all of the base-labile protecting groups described here, Fm is incompatible with Fmoc SPPS. ${ }^{123}$ Some Fm-protected peptides can display poor solubility in polar media, and a low peptide yield is observed upon final HF cleavage from the resin when the $C$-terminal amino acid is Cys(Fm). Dnpe is an alternative protecting group that may be used to avoid these complications. ${ }^{124}$

5.4.2 2-(2,4-Dinitrophenyl)ethyl (Dnpe). 2-(2,4-Dinitrophenyl)ethyl (Dnpe) was introduced in 1992 as a replacement for the Fm group in Boc SPPS (Fig. 22b). ${ }^{124}$ Compared to Fm, superior yields were obtained following HF cleavage when the C-terminal amino acid is Cys; this is attributed to the reduced steric hinderance encountered during cleavage when using Dnpe. Dnpe is more base-labile than Fm and cleavage using 50\% piperidine in DMF can yield the desired disulfide-containing peptide within $30 \mathrm{~min}$. Alternatively, the free thiol-containing peptide can be obtained within $1 \mathrm{~h}$ if this process is done in the presence of $2 \% \mathrm{BME}$ under an argon atmosphere. ${ }^{124}$ Deprotection can also be accomplished using DBU (2\% v/v in DMF). ${ }^{68}$

Dnpe is stable to a wide variety of reagents, both basic and acidic. These include: 5\% DIEA in DCM ( $2 \mathrm{~h}), 40 \%$ TFA in DCM (24 h), 90\% HF in the presence of $p$-cresol or anisole $\left(1 \mathrm{~h}, 0{ }^{\circ} \mathrm{C}\right)$, and TFMSA- $p$-cresol in TFA $\left(1: 3: 10,2 \mathrm{~h}, 25^{\circ} \mathrm{C}\right)$. It is also stable to oxidative conditions, e.g. $\mathrm{Tl}(\mathrm{TFA})_{3}$ in $\mathrm{TFA}, \mathrm{I}_{2}$ in $80 \% \mathrm{AcOH}$ (aq.) and is thus orthogonal to protecting groups including Meb, Acm and StBu. ${ }^{124}$

5.4.3 9-Fluorenylmethyloxycarbonyl (Fmoc). Although widely used for N-protection of the $\alpha$-amino group in SPPS, it was not until 2001 that 9-fluorenylmethyloxycarbonyl (Fmoc) as a protecting group for the Cys thiol was demonstrated (Fig. 22c). ${ }^{125}$ Cys(Fmoc) can be converted to Cys(Fm) by treatment with $\mathrm{Et}_{3} \mathrm{~N}$. Fm may then be removed as described previously. Furthermore, $S$-Fmoc can be removed preferentially over $N$-Fmoc through choice of base, allowing the two groups to be used together. Although Fmoc still has not become widely used as an $S$-protecting group, it provides a simple way of synthesising Fm derivatives. Additionally, $S$-Fmoc could be useful in synthetic strategies that require selective deprotection of the thiol group in the presence of $\mathrm{N}$-Fmoc. ${ }^{125}$

\subsection{Enzyme-labile protecting groups}

5.5.1 Phenylacetamidomethyl (Phacm). Phenylacetamidomethyl (Phacm, Fig. 23a) is an environmentally-friendly alternative to Acm, first described in 1991 by Greiner and Hermann. ${ }^{221}$ The protecting group was then introduced to SPPS in 1995 by Royo et $a .^{126}$ Phacm has very similar stability and lability to Acm but is additionally cleaved by $E$. coli penicillin G acylase (PGA, E.C. 3.5.1.1). ${ }^{126}$ PGA can be immobilised on amino-acrylic resin (iPGA, Fig. 23b), which allows the enzyme to be recycled for repeated use; total enzymatic activity is still retained after five reaction cycles. Immobilisation of the enzyme is beneficial as it decrease the environmental impact and increase the cost-effectiveness of the enzyme. Immobilised enzymes also tend to have enhanced stability and display activity over a wider range of conditions. ${ }^{127} \mathrm{Cys}(\mathrm{Phacm})$ deprotection can be performed under mild conditions; the optimal conditions for using iPGA are $0.1 \mathrm{mM}$ phosphate buffer, $\mathrm{pH}$ 7.9, in
DMSO (95:1), at $37{ }^{\circ} \mathrm{C}$. DMSO is the best-tolerated solvent, but enzymatic activity remains almost completely intact when DMSO is replaced by any of a variety of organic co-solvents: $\mathrm{MeCN}, \mathrm{DMF}$, EtOH, various other alcohols and $\mathrm{Et}_{2} \mathrm{O}$. The $\mathrm{pH}$ range under which deprotection can occur is also broad, with clean removal still observed at pH 5.9. ${ }^{127}$ If enzymatic hydrolysis is performed in the presence of BME, the free thiol is obtained, otherwise, the Cys is oxidised directly to the disulfide. ${ }^{126}$ The efficiency of iPGA can depend on the peptide sequence containing Cys(Phacm), as some peptide sequences appear to tolerate a broader range of conditions than others. Care must therefore be taken to choose the optimal conditions for a given sequence. ${ }^{127}$ Phacm may also be removed using $\mathrm{Hg}$ (II) or $\mathrm{Ag}$ (II) salts, or under conventional oxidative conditions, e.g. using $\mathrm{I}_{2}$ or $\mathrm{Tl}(\mathrm{TFA})_{3}$. However, removal under enzymatic conditions circumvents the problems with such reagents, as there is no need to use toxic heavy metals or reagents (e.g. $\mathrm{I}_{2}$ ) which can favour peptide chain modifications (e.g. back alkylation), have difficult to remove residual products and are damaging to the environment. ${ }^{127}$ Phacm is stable to acidic and basic conditions, including 5\% DIEA in DCM, 40\% TFA in DCM, 25\% piperidine in DMF, $0.1 \mathrm{M}$ TBAF in DMF and 5\% DBU in DMF (all for $24 \mathrm{~h}$ at $25^{\circ} \mathrm{C}$ ). Phacm is also stable to $90 \% \mathrm{HF}$ in the presence of anisole or $p$-cresol $\left(1 \mathrm{~h}, 0{ }^{\circ} \mathrm{C}\right)$ and $90 \%$ TFA in the presence of a number of scavengers (i.e. phenol, ethane-1,2-dithiol (EDT), $p$-cresol, anisole, $2 \mathrm{~h}, 25{ }^{\circ} \mathrm{C}$ ). This makes Phacm suitable for both Fmoc and Boc SPPS. However, it is more useful for Fmoc SPPS, as it is partially cleaved $(<20 \%)$ by TFMSA-TFA- $p$-cresol $\left(1: 10: 0.3,2 \mathrm{~h}, 25{ }^{\circ} \mathrm{C}\right) .{ }^{126}$ Phacm is orthogonal to a wide variety of protecting groups, including Fm, Dnpe, Meb, Trt and Tmob. ${ }^{126}$ In addition to those mentioned, Phacm is orthogonal to many acid-labile protecting groups. ${ }^{127}$ It is also partially orthogonal to Acm, provided Phacm is removed enzymatically prior to Acm deprotection. ${ }^{126}$ This has been demonstrated on model tripeptides containing Cys(Phacm) and Cys(Acm) (Fig. 23c). ${ }^{127}$ Cys(Phacm) derivatives, which have been used as protecting groups/solubilising tags in the chemical synthesis of proteins such as histone $\mathrm{H} 4$, can also be removed using $\mathrm{PdCl}_{2}$ under aqueous conditions. ${ }^{222}$ Additionally, the orthogonality of Cys(Phacm) can be used to facilitate multiinterchain disulfide formation in the final step of three-disulfide containing peptides synthesis, such as in the $\mathrm{I}_{2}$ free synthesis of a porcine insulin analogue (Fig. 23d). ${ }^{223}$ Here, the A chain (containing four c) can be synthesised using a combination of Cys(S-Tmp), Cys(Mmt), Cys(Trt) and Cys(Phacm). Selective deprotection, followed by activation, yields the Cys(Trt), Cys(Phacm), disulfide containing resin bound A chain. Simultaneous Trt removal and resin cleavage could then be achieved with a cleavage cocktail of TFA: TIS : $\mathrm{H}_{2} \mathrm{O}(92.5: 5: 2.5)$. Incubation of this A chain peptide with a Cys(Phacm), Cys(5-Npys) activated B chain peptide in ligation buffer $(0.2 \mathrm{M} \mathrm{NH} 4 \mathrm{OAc}, 6 \mathrm{M}$ urea, $\mathrm{pH} 4.5)$ lead to formation of one of the interchain disulfides. Finally, selective removal of the Phacm protecting group with immobilised PGA and activation with 3 equiv. 5,5'-dithiobis-(2-nitrobenzoic acid) (DTNB) lead to formation of the second interchain disulfide to give the desired product. Optimisation of activation with DTNB proved critical to minimise misfolding and disulfide scrambling; 
a) Phenylacetamidomethyl<smiles>CC(C)NC(=O)[C@H](CSCNC(=O)Cc1ccccc1)NC(C)C</smiles>

b) Immobilised Penicillin G Acylase (iPGA)

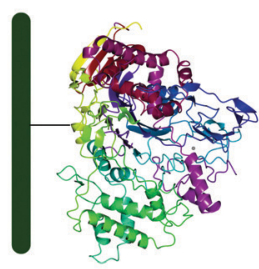

c) Orthogonal Phacm and Acm deprotection<smiles></smiles>

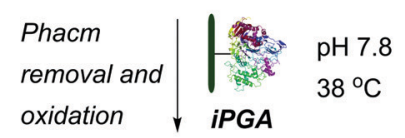

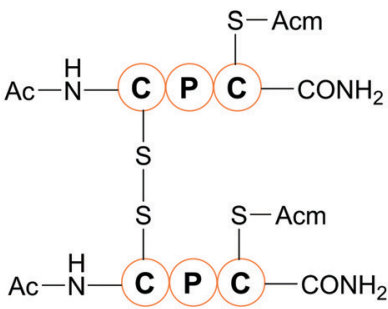

\begin{tabular}{l|l} 
Acm & $\begin{array}{l}\mathrm{I}_{2} \\
\text { removal and } \\
\text { oxidation }\end{array}$
\end{tabular} \mid $\begin{aligned} & \mathrm{AcOH}: \mathrm{H}_{2} \mathrm{O} \\
& (4: 1)\end{aligned}$

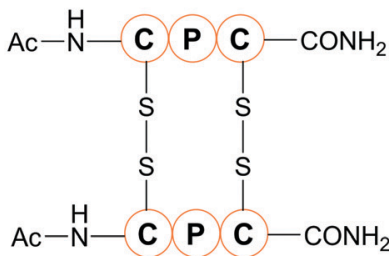

d) Synthesis of ValA16, MetB1, MetB17-porcine insulin

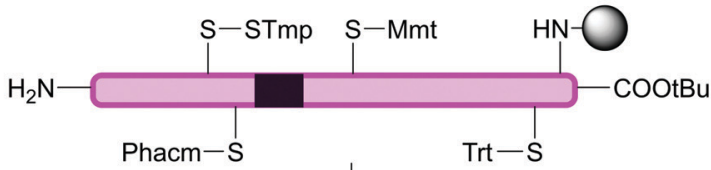

i) STmp deprotection

ii) Thiol activation

iii) Mmt deprotection

BME in DMF

+ disulfide formation

ii) DTNB in DMF

iii) 1:5:94 (TFA:TIS:DCM)

iv) $97.5: 5: 2.5$ (TFA:TIS: $\mathrm{H}_{2} \mathrm{O}$ )

iv) Resin cleavage
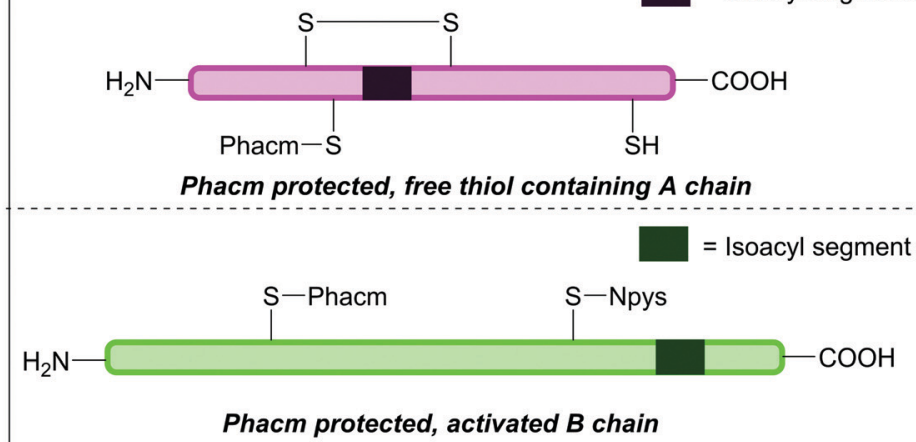

\begin{tabular}{l|l} 
i) Dimer formation & i) $0.2 \mathrm{M} \mathrm{NH}_{4} \mathrm{OAC}, 6 \mathrm{M}$ urea, $\mathrm{pH} 4.5$
\end{tabular}

ii) $\mathrm{O}->\mathrm{N}$ shift of isoacyl Phacm protected, free thiol segments containing A chain

ii) $50 \mathrm{mM}$ Tris $\mathrm{pH} 8.0$
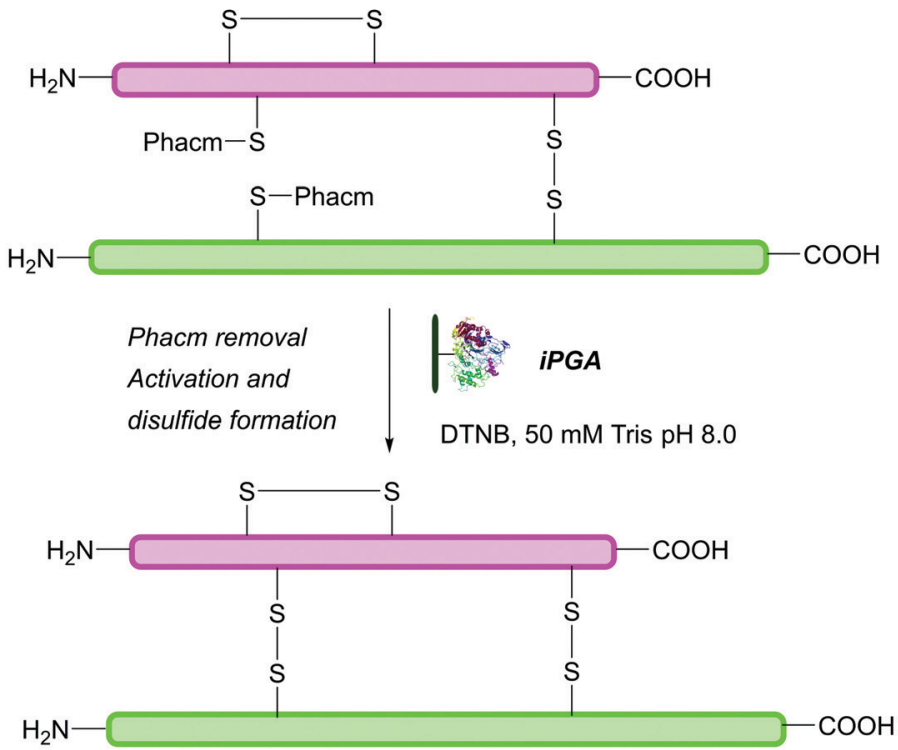

ValA16, MetB1, MetB17-porcine insulin

$=-$ GIVEQCCTSICSLYQVENYCNN-

= -MVNQHLCGSHLVEALYMV CGERGFFYYTPKA-

Fig. 23 (a) Cys thiol protection with the phenylacetamidomethyl (Phacm) protecting group. (b) Schematic of immobilised Penicillin G Acylase (iPGA) used for Cys(Phacm) deprotection. (c) Orthogonal deprotection of Cys(Phacm) and Cys(Acm) in a model tripeptide. (d) Synthesis of a porcine insulin analogue using Cys(Phacm). 
oxidation with no DTNB or in $10 \%$ DMSO led to only scrambled side products, whereas double-DTNB side products were observed when using $>4$ equiv. DTNB. ${ }^{223}$ A similar strategy was adopted for the synthesis of human relaxin-2 using Cys(Phacm) and Cys $(t \mathrm{Bu})$; here, DTNB was replaced with activating agent bis(5(2-methoxyethoxy)-2-pyrimidinyl disulfide (BMPD) post-Phacmremoval to give an improved peptide yield. ${ }^{224}$

\subsection{Hydrazine-labile protecting groups}

5.6.1 Hydroxyglycine-Acm (Hgm) and hydroxyquinoline-Acm (Hqm). Hydroxyglycine-Acm (Hgm, Fig. 24a) and hydroxyquinoline-Acm (Hqm, Fig. 24b) were devised by Shen et al. in $2011 .^{128}$ Both groups are stable to Fmoc and Boc SPPS and are removable by hydrazine $\left(5 \% \mathrm{v} / \mathrm{v}\right.$ in $\left.\mathrm{H}_{2} \mathrm{O}\right)$ under mild conditions

a) Hqm

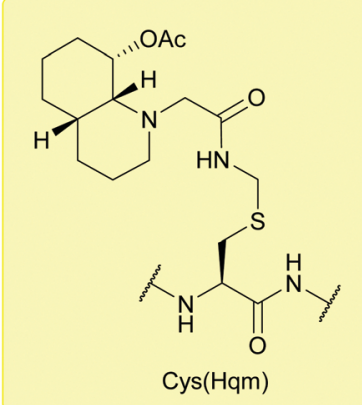

$\mathrm{H}=$ Hydroxy, $\mathrm{q}=$ quinoline, $\mathrm{g}=$ glycine, $\mathrm{m}=$ Derived from $\mathrm{Ac} \underline{\mathrm{m}}$

c) Synthesis of human neutrophil defensin (hNP2)

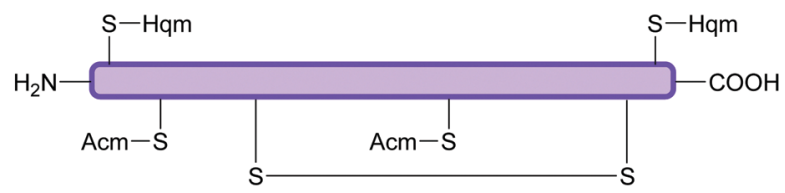

\begin{tabular}{l|l} 
Hqm deprotection & $\begin{array}{c}\text { i) } 5 \% \mathrm{v} / \mathrm{v} \text { hydrazine, } \mathrm{H}_{2} \mathrm{O}: \mathrm{MeCN}(1: 1) \\
\mathrm{pH} 8.5,37^{\circ} \mathrm{C}, 24 \mathrm{~h}\end{array}$ \\
Disulfide formation & $\begin{array}{c}\text { ii) } 20 \% \mathrm{DMSO}, \mathrm{H}_{2} \mathrm{O}: \mathrm{MeCN}(1: 1), \mathrm{pH} 6.0,24 \mathrm{~h}\end{array}$
\end{tabular}
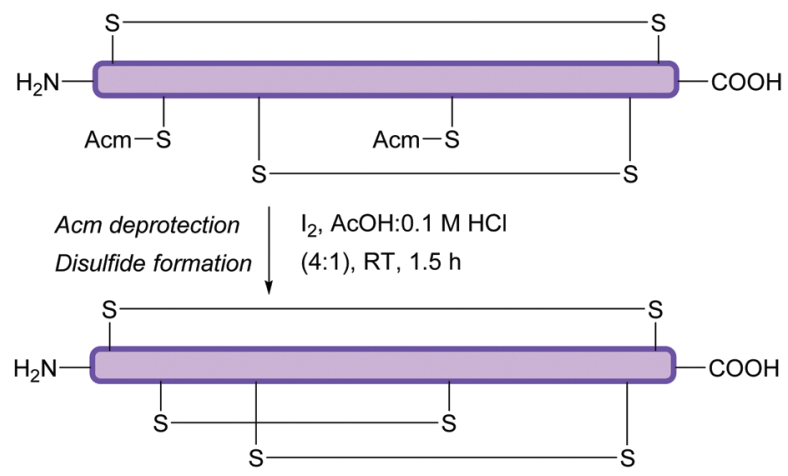

Human neutrophil defensin (hNP2)

$=-\underline{C} Y \underline{C} R I P A \underline{C} \| G E R R Y G T \underline{C} I Y Q G R L W A F \underline{C C}-$

Fig. 24 (a) Cys thiol protection with the Hqm protecting group. (b) Cys thiol protection with the Hgm protecting group. (c) Synthesis of human neutrophil defensin (hNP2) using Cys(Phacm).
$\left(\mathrm{pH} 8.5,37^{\circ} \mathrm{C}\right)$. In both cases, hydrazine removes the OAc moiety, triggering an internal deprotection step. Hqm is removed within $8 \mathrm{~h}$, while Hgm takes $72 \mathrm{~h}$ for full removal; Hqm is therefore recommended for practical use. Treatment with standard oxidative reagents (i.e. $\mathrm{I}_{2}$ or $\mathrm{AgOAc}$ ) removes $\mathrm{Hqm}$ within $30 \mathrm{~min}$. Hqm can be considered orthogonal to (and subsequently removed in the presence of) Acm due to the latter's stability towards treatment with hydrazine. This orthogonality was demonstrated in a synthesis of human neutrophil defensin hNP2 using Hqm, Acm and Trt as three orthogonal protecting groups (Fig. 24c). An improved final yield of the peptide was observed when compared to previous reports that used the $\mathrm{StBu}, \mathrm{Acm}$ and Trt groups. ${ }^{128}$

\subsection{Palladium-labile protecting groups}

5.7.1 Allyloxycarbonyl (Alloc). The allyloxycarbonyl (Alloc) group has been previously described for the protection amino acids, including the thiol group of Cys (Fig. 25a). ${ }^{225}$ Alloc groups can be deprotected using tributyltin hydride $\left(\mathrm{Bu}_{3} \mathrm{SnH}\right)$ and a $\operatorname{Pd}(0)$ catalyst. Removal can be achieved within $10 \mathrm{~min}$ using $\mathrm{PdCl}_{2}\left(\mathrm{PPh}_{3}\right)_{2}$ in DCM and $\mathrm{AcOH}$ with $\mathrm{Bu}_{3} \mathrm{SnH}$. The Alloc group is stable in TFA/DCM $\left(24 \mathrm{~h}, 50{ }^{\circ} \mathrm{C}\right)$, but base-labile piperidine treatment ( $30 \%$ in DMF, $3 \mathrm{~h}, 30{ }^{\circ} \mathrm{C}$ ) of Boc-Cys(Alloc)$\mathrm{OH}$ results in complete removal of the Alloc group. Additionally, under Fmoc conditions, the Alloc group is prone to undergoing $\beta \rightarrow \alpha$ shifts, and intramolecular acylation reactions may also occur. ${ }^{129}$ These issues associated with the Alloc group hinder its suitability as a Cys protecting group. As such, other allyl-based Pd-labile protecting groups, such as Allocam, are recommended instead. $^{130}$

5.7.2 Allyloxycarbonylaminomethyl (Allocam). The allyloxycarbonylaminomethyl (Allocam) group was reported in 1994 as a replacement for Alloc in Cys protection chemistry (Fig. 25b). ${ }^{130}$ The Allocam group can be removed using $\mathrm{Bu}_{3} \mathrm{SnH}$ and $\operatorname{Pd}(0)$ catalyst in $\mathrm{AcOH}$ (10 min, RT). Following deprotection, the crude reaction mixture contains a mixture of the tributyltin salt, the free thiol, and additionally minor amounts of the corresponding disulfide. 0.5 equivalents of $\mathrm{I}_{2}$ may then be added to isolate the deprotected Cys residues as their disulfide derivatives. ${ }^{130}$ In the presence of $\mathrm{Pd}$, Cys(Allocam) derivatives can rearrange into allyl thioethers with a corresponding loss of $\mathrm{CO}_{2}$ and methylenimine. This is prevented by adding a high concentration of a weak nucleophilic species, such as $\mathrm{AcOH}$, into the reaction to trap the $\pi$-allyl entity prior to rearrangement. Without addition of $\mathrm{AcOH}$, $\sim 5-10 \%$ formation of allyl thioethers is observed. ${ }^{130}$ Cys(Allocam) displays stability towards piperidine, ${ }^{226}$ but is slightly unstable to the acidic conditions used for Boc removal, ${ }^{227}$ with $\sim 10 \%$ degradation seen following $20 \mathrm{~h}$ of treatment with $25 \%$ TFA in DCM. ${ }^{130}$

After its initial documentation, the Allocam group saw little use as a protecting group for Cys in the literature. More recently, however, it has seen a revival as an orthogonal Cys protecting group. Here, alternative deprotection protocols that lead directly to the disulfide formation on-resin have been developed: $\mathrm{Pd}(\mathrm{OAc})_{2}$ (1.5 equiv.), $3 \% \mathrm{NMM}$ and $5 \% \mathrm{AcOH}$ in DMSO for $2 \mathrm{~h}$. Under these conditions, complete removal of Allocam could be achieved to yield the disulfide-containing 
a) Allyloxycarbonyl

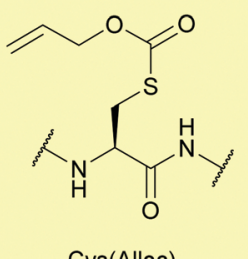

Cys(Alloc)

b) Allyloxycarbonylaminomethyl

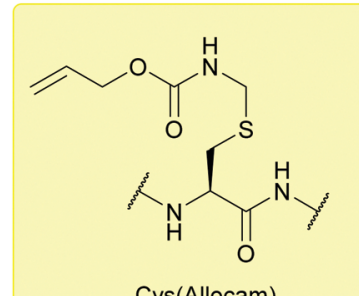

Cys(Allocam)

c) Synthesis of $\alpha 4 / 7-$ Conotoxin LvIA ( $\alpha$-LvIA)
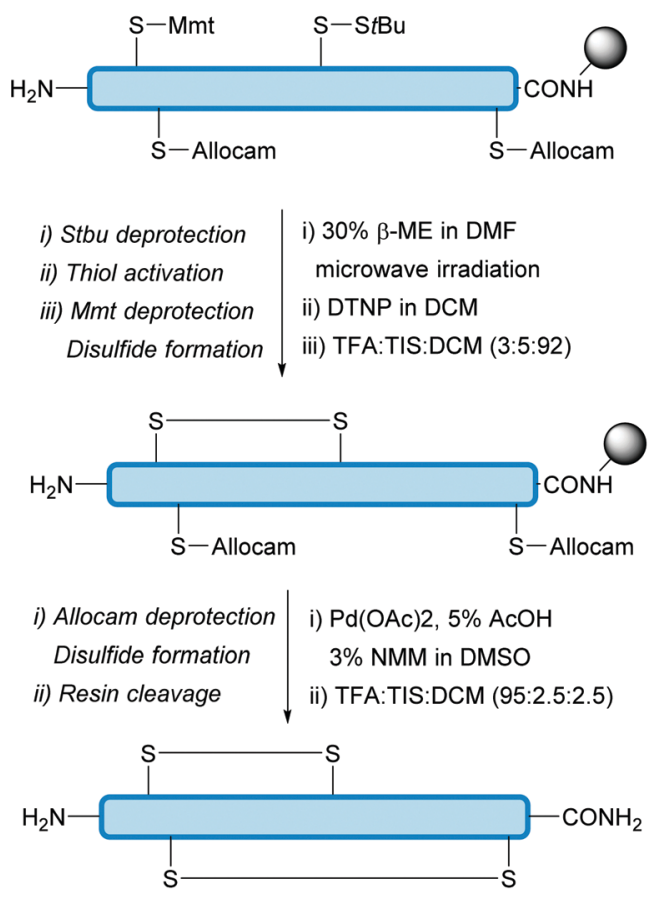

$\alpha 4 / 7$-Conotoxin LvIA ( $\alpha$-LVIA)

$=-$ GCCSHPACNNDHPEI $\underline{\mathrm{C}}-$

Fig. 25 (a) Cys thiol protection with the allyloxycarbonyl (Alloc) protecting group. (b) Cys thiol protection with the allyloxycarbonylaminomethyl (Allocam) protecting group. (c) Synthesis of $\alpha 4 / 7$ Conotoxin LvIA ( $\alpha$-LvlA) using Cys(Allocam).

resin-bound peptide. ${ }^{131}$ The use of Cys(Allocam) has since been expanded towards higher-yielding, on-resin synthesis of $\alpha 4 / 7$-Conotoxin LvIA ( $\alpha$-LvIA, Fig. 25c). ${ }^{205}$ Orthogonality of Cys(Allocam) to other protecting groups was also further established.
For example, treatment of peptides containing Cys(Trt) and Cys $(t \mathrm{Bu})$ with $\mathrm{Pd}(\mathrm{OAc})_{2}$ (1.5 equiv.), $3 \% \mathrm{NMM}$ and $5 \% \mathrm{AcOH}$ in DMSO for $2 \mathrm{~h}$, followed by resin cleavage, led to no removal of either protecting group, whereas treatment of Cys(Mmt) with the same conditions gave a mixture of products. Peptides containing two Cys(Allocam) residues could be fully deprotected to give the corresponding disulfide when using $\mathrm{I}_{2}$, whereas the Allocam protecting groups remained intact when using conditions typically used for $\mathrm{StBu} /$ Mmt removal (20\% BME, DTNP, 1\% TFA, 5\% TIS). Depending on the combination of Cys protecting groups used, Cys(Allocam) could be orthogonally deprotected first or last in a given sequence to perform regioselective synthesis of disulfide-containing $\alpha$-LvIA on-resin. ${ }^{205}$

5.7.3 $\left[N\right.$-[2,3,5,6-Tetrafluoro-4-( $N^{\prime}$-piperidino)-phenyl], $N$-allyloxycarbonyl]-aminomethyl (Fnam). The $[N-[2,3,5,6$-tetrafluoro-4( $N^{\prime}$-piperidino)-phenyl], $N$-allyloxycarbonyl]-aminomethyl (Fnam) group (Fig. 26a) was synthesised in 1999, whilst looking for a replacement to the Allocam group that was more stable to TFA. ${ }^{132}$ The decomposition of Allocam in the presence of acid was hypothesised to be an acid-catalysed fragmentation reaction, which led to the formation of a reactive acyliminium cation. One way which was theorised to increase the stability of Allocam to TFA was to add an electron-withdrawing group to the nitrogen atom, which would disfavour acyliminium formation. Fnam can be removed using $\operatorname{Pd}(0)$ complexes in the presence of allyl scavengers, e.g. $\mathrm{Bu}_{3} \mathrm{SnH}$ in $\mathrm{DCM}$ in the presence of $5 \mathrm{~mol} \% \mathrm{PdCl}_{2}\left(\mathrm{PPh}_{3}\right)_{2}$ and $\mathrm{AcOH}$, followed by $\mathrm{AcOH} / \mathrm{BME}$ to obtain the free thiol. Alternatively, following up with $\mathrm{I}_{2}$ treatment will give the disulfide. $\mathrm{Pd}\left(\mathrm{PPh}_{3}\right)_{4}$ in the presence of either $\mathrm{PhSiH}_{3}$ or NDMBA also caused deprotection within $15 \mathrm{~min}$ and $60 \mathrm{~min}$, respectively (these systems fail to deprotect $S$-Allocam). ${ }^{132}$ As with their Allocam homologues, a scavenger $\left(\mathrm{Bu}_{3} \mathrm{SnH}, \mathrm{PhSiH}_{3}\right.$ or NDMBA) is needed to avoid rearrangement to allyl thioethers. Fnam is completely acid and base stable, and is compatible with both Boc and Fmoc SPPS. Fnam has thus been hypothesised to be compatible with most acid- and base-labile protecting groups. However, Fnam is not stable to the deprotection conditions used for Acm, i.e. heavy metal salts or oxidants such as $\mathrm{Tl}(\mathrm{TFA})_{3}{ }^{132}$

5.7.4 $S$-[ $N$-[2,3,5,6-Tetrafluoro-4-(phenylthio)-phenyl], $N$-allyloxycarbonyl]-aminomethyl (Fsam). $S$-[N-[2,3,5,6-Tetrafluoro-4(phenylthio)-phenyl], $N$-allyloxycarbonyl]-aminomethyl (Fsam, Fig. 26b) is an easier to handle analogue of Fnam. ${ }^{133}$ Conditions for its removal are identical: a $\operatorname{Pd}(0)$ complex in the presence of allyl scavengers, e.g. $\mathrm{PhSiH}_{3}$ (or NDMBA) in DCM, followed by $\mathrm{Pd}\left(\mathrm{PPh}_{3}\right)_{4}$ (inert atmosphere, $10 \mathrm{~min}, 25{ }^{\circ} \mathrm{C}$ ). This gives a mixture of the free thiol and thioaminals - $\mathrm{AcOH} / \mathrm{BME}$ can then be added to give the free thiol as the sole product. As with Fnam, $\mathrm{PdCl}_{2}\left(\mathrm{PPh}_{3}\right)_{2} / \mathrm{Bu}_{3} \mathrm{SnH}$ can also be used, but is more difficult to handle. $\mathrm{I}_{2}$ may be added to remove the Fsam protecting group, yielding a disulfide bond. ${ }^{133}$

Fsam is stable to acidic and basic conditions, and is compatible with both Boc and Fmoc SPPS. Fsam is labile to similar oxidative conditions to Acm and Phacm. A side reaction was observed during SPPS, which was suggested to be due to $\mathrm{N}$-allyl peptides forming via nucleophilic attack of the neighbouring $\alpha$-amino function at the allyl group. This reaction was proposed 
a) $[N-[2,3,5,6-T e t r a f l u o r o-4-(N '-p i p e r i d i n o)-p h e n y l]$, $\mathrm{N}$-allyloxycarbonyl]-aminomethyl

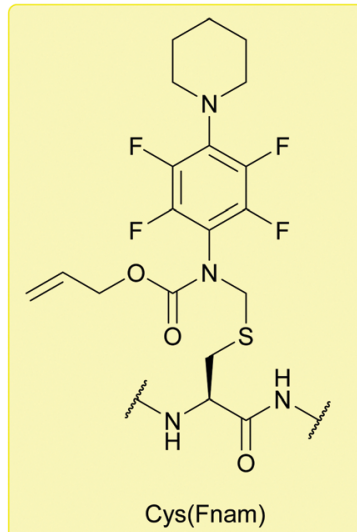

b) $S$-[N-[2,3,5,6-Tetrafluoro-4-(phenylthio)-phenyl], $\mathrm{N}$-allyloxycarbonyl]-aminomethyl

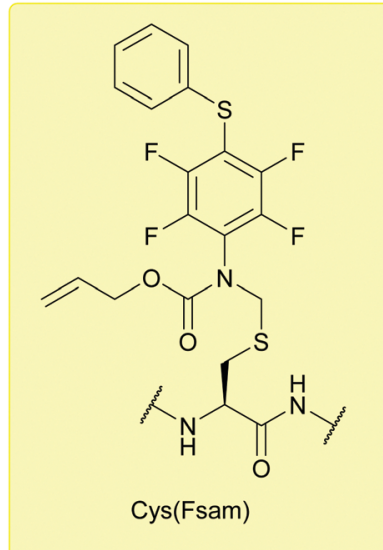

Fig. 26 (a) Cys thiol protection with the $\left[N-\left[2,3,5,6\right.\right.$-tetrafluoro-4- $\left(N^{\prime}-\right.$ piperidino)-phenyl], $\mathrm{N}$-allyloxycarbonyl]-aminomethyl (Fnam) (b) Cys thiol protection with the $S$-[N-[2,3,5,6-tetrafluoro-4-(phenylthio)-phenyl], $N$ allyloxycarbonyl]-aminomethyl (Fsam) protecting group.

to occur under the basic conditions present during Fmoc removal or during the coupling step. This phenomenon has also been observed with the Alloc group. ${ }^{133}$

5.7.5 Allyl (Sac). S-Allyl-cysteine (Cys(All), Sac, Fig. 27a) is a protected Cys variant that is naturally found in garlic. ${ }^{228}$ Recently it has been shown that Sac can be incorporated via unnatural amino acid mutagenesis into Cys-free superfolder GFP (cfsfGFP(R2Sac)). ${ }^{134}$ In contrast to other UAA incorporation strategies, which rely on addition of the UAA to the growth medium during protein biosynthesis, Sac incorporation was achieved by addition of allyl mercaptan; this precursor could be biosynthetically converted to Sac, and subsequently incorporated into the target GFP. The allyl group can be subsequently deprotected overnight using $\operatorname{Pd}(\mathrm{TPPTS})_{4}$ followed by addition of DTT to yield the free thiol-containing GFP in PBS (Fig. 27b). The allyl group could further be used in thiol-ene chemistry to fluorescently label hydrogels with cfsfGFP(R2Sac). ${ }^{134}$

5.7.6 $S$-Propargyl-cysteine (SprC). $S$-Propargyl-cysteine (SprC) is a Cys derivative where the thiol has been capped with a a) AllyI
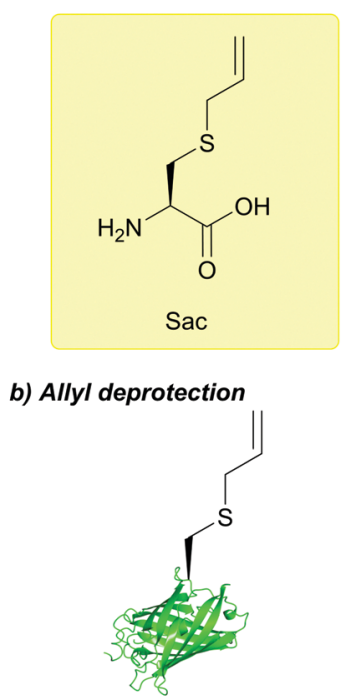

Sac-containing cysteine-free superfolder GFP cfsfGFP(R2Sac)

\begin{tabular}{l|l} 
i) Pd(TPPTS $)_{4}$ & ii) DTT \\
PBS pH 7.4 & PBS pH 7.4 \\
Overnight & $20 \mathrm{~min}$ \\
$37^{\circ} \mathrm{C}$ & $37^{\circ} \mathrm{C}$
\end{tabular}

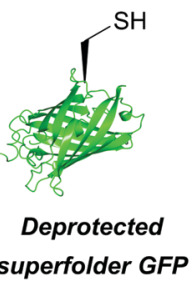

Fig. 27 (a) S-allyl cysteine (Sac). (b) Pd-mediated deprotection of Saccontaining cysteine-free superfolder GFP (cfsfGFP(R2Sac)).

propargyl group (Fig. 28a). Similarly to Sac, SprC has been used in unnatural amino acid mutagenesis to generate proteins bearing a protected Cys, such as SprC-containing human thioredoxin (Trx73SprC). SprC could then be deprotected with Pd(TPPTS $)_{4}$ followed by DTT addition to give the free-thiol containing protein (in PBS, pH 7.4, $37{ }^{\circ} \mathrm{C}$ ). Control of catalytic Cys activity via SprC Cys protection/deprotection could also be achieved, as shown with SprC-containing human rhinovirus-14 3C (HRV 3C(147SprC)) protease. HRV 3C(147SprC) protease showed no proteolytic activity against substrates containing a relevant HRV 3C protease cleavage site; upon in situ addition of Pd however, substrate cleavage was initiated as confirmed by SDS-PAGE analysis, suggesting activation of protease activity and thus deprotection of the catalytic Cys (Fig. 28b). This was further confirmed by ESI-MS analysis. SprC-containing proteins could additionally undergo bioconjugation reactions using the alkyne group as a reactive handle, including thiol-yne click reactions, and $\mathrm{Cu}(\mathrm{I})$-catalysed azide-alkyne cycloaddition (CuAAC). In particular, SprC can participate in Pd-catalysed Sonogashira coupling, the product of which can be cleaved/deprotected with a different 
Pd catalyst to give the free Cys containing protein. For example, Sonogashira coupling could be performed between SprC-containing enhanced GFP (EGFP(182SprC)) and biotin-iodobenzene using $\mathrm{Pd}\left(\mathrm{NO}_{3}\right)_{2}$ to yield biotinylated GFP; this species could then be deprotected with $\operatorname{Pd}(\mathrm{TPPTS})_{4}$ to release the conjugated biotin and reveal EGFP(182Cys, Fig. 28c).

5.7.7 Succinimide (Suc). The reaction of maleimides with Cys to yield succinimide (Suc) conjugates is one of the most widely used methods for a variety of applications, such as surface modification, ${ }^{229}$ ADC synthesis, ${ }^{230}$ and fluorescent bioconjugates. ${ }^{231}$ Very recently, Suc has been applied as a Pd-labile Cys protecting group (Fig. 29a) for use in protein semisynthesis. ${ }^{136}$ Suc undergoes deprotection using $\mathrm{PdCl}_{2}$ similar to $t \mathrm{Bu}$ and $\mathrm{Acm}$, with deprotection significantly accelerated by addition of $\mathrm{MgCl}_{2}$ (giving total Suc deprotection within $45 \mathrm{~min}$ as opposed to $4 \mathrm{~h}$ ). Additionally, Cys(Suc) remains stable to treatment with $\left[\mathrm{Pd}(\right.$ allyl $\left.) \mathrm{Cl}_{2}\right]$, giving a degree of orthogonality to $\mathrm{Thz}$ (Thz is partially labile to $\mathrm{PdCl}_{2}$ treatment). Furthermore, Cys(Suc) shows stability to conditions both for NCL and desulfurisation, as demonstrated in the synthesis of disulfide-containing Trx-1. Here, an N-terminal fragment of Trx-1 (containing a $C$-terminal thioester) was synthesised via expressed protein ligation (EPL), and the two Cys residues capped with $\mathrm{N}$-methyl maleimide for Cys(Suc) protection. Capping was carried out at $-18{ }^{\circ} \mathrm{C}$ to avoid hydrolysis of the succinimide ring. Subsequent NCL of a C-terminal fragment of Trx-1 (containing an $\mathrm{N}$-terminal Cys), followed by desulfurisation, led to the Cys(Suc) protected Trx-1. Deprotection of Cys(Suc) with $\mathrm{PdCl}_{2}$, followed by oxidation with DTC yielded the desired disulfide-containing Trx-1 (Fig. 29b). Maleimides for Suc protection of Cys can also be functionalised for further applications; for example, a Suc-based protecting group could act both as a protecting group and a linker to a solid phase resin (PEGA resin) in the synthesis of ubiquitin activity-based probes. ${ }^{136}$

\subsection{N-terminal cysteine protecting groups}

5.8.1 Thiazolidine (Thz). The thiazolidine group (Thz) was introduced as far back as 1937, where it was noted that formaldehyde reacts with Cys residues to form thiazolidinecarboxylic

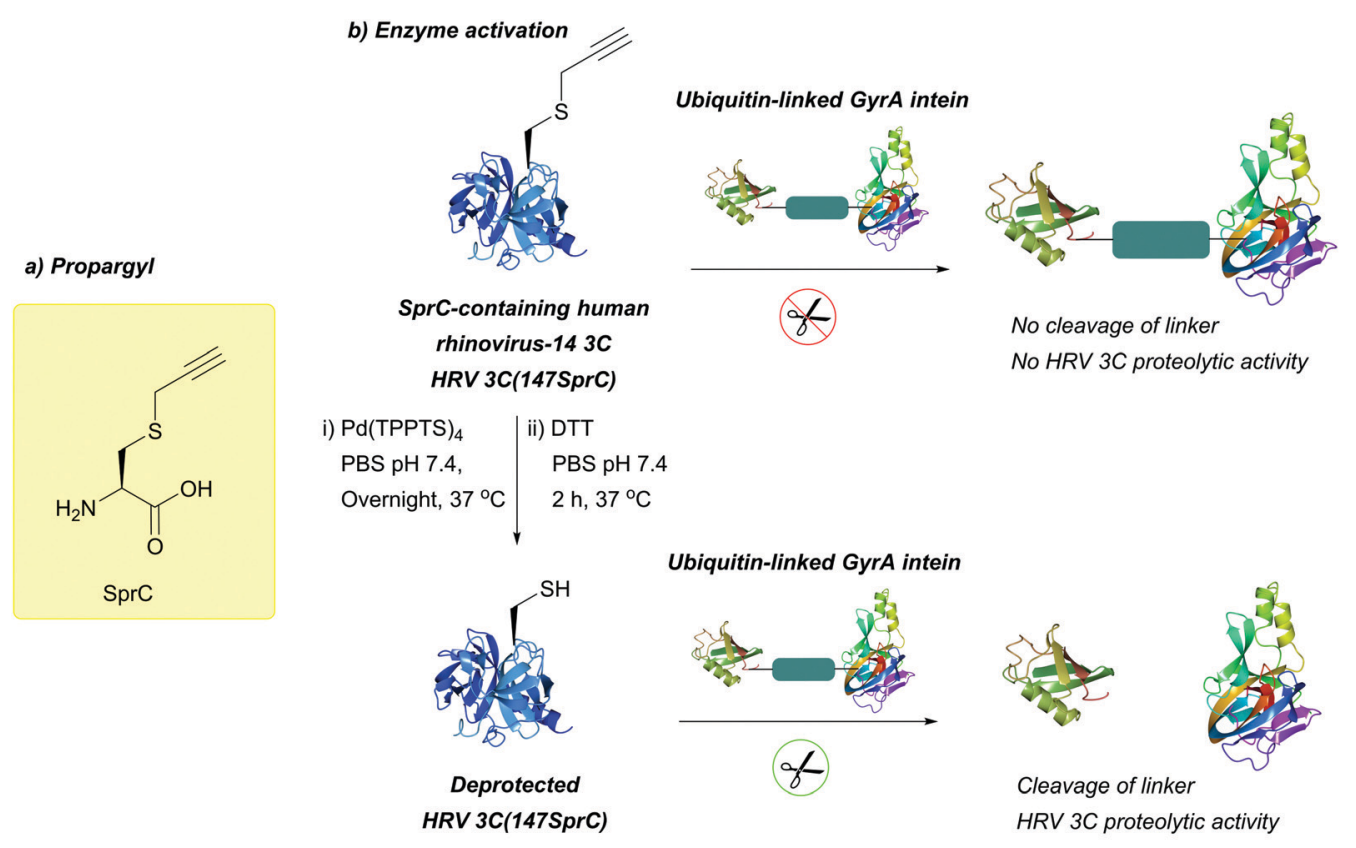

c) SprC conjugation and deprotection

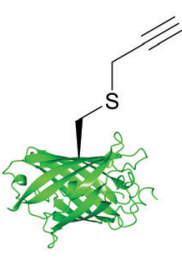

SprC-containing

enhanced GFP EGFP(182SprC)
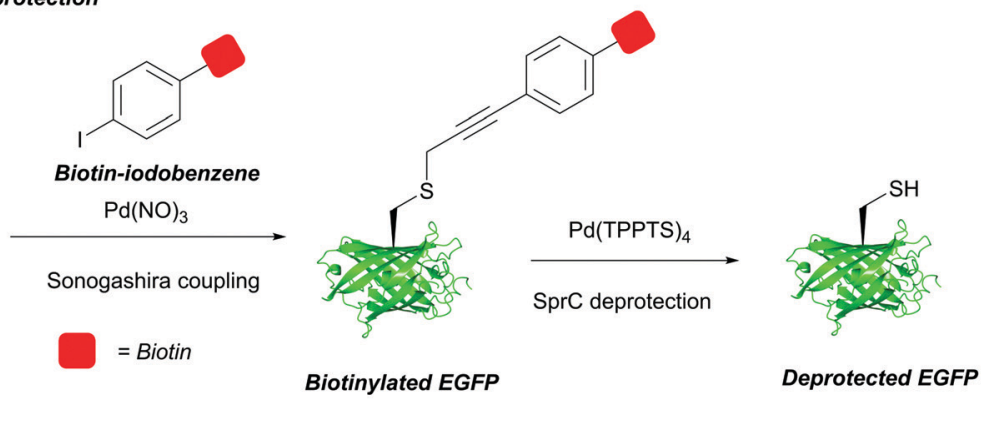

Fig. 28 (a) S-Propargyl-cysteine (SprC). (b) Pd-Mediated deprotection of SprC-containing human rhinovirus-14 3C (HRV 3C(147SprC)). Upon SprC deprotection, HRV $3 \mathrm{C}$ proteolytic activity is restored. (c) Sonogashira coupling of SprC-containing enhanced GFP (EGFP(182SprC)), followed by subsequent Pd-mediated deprotection to yield the free-thiol containing EGFP. 
b) Synthesis of thioredoxin-1

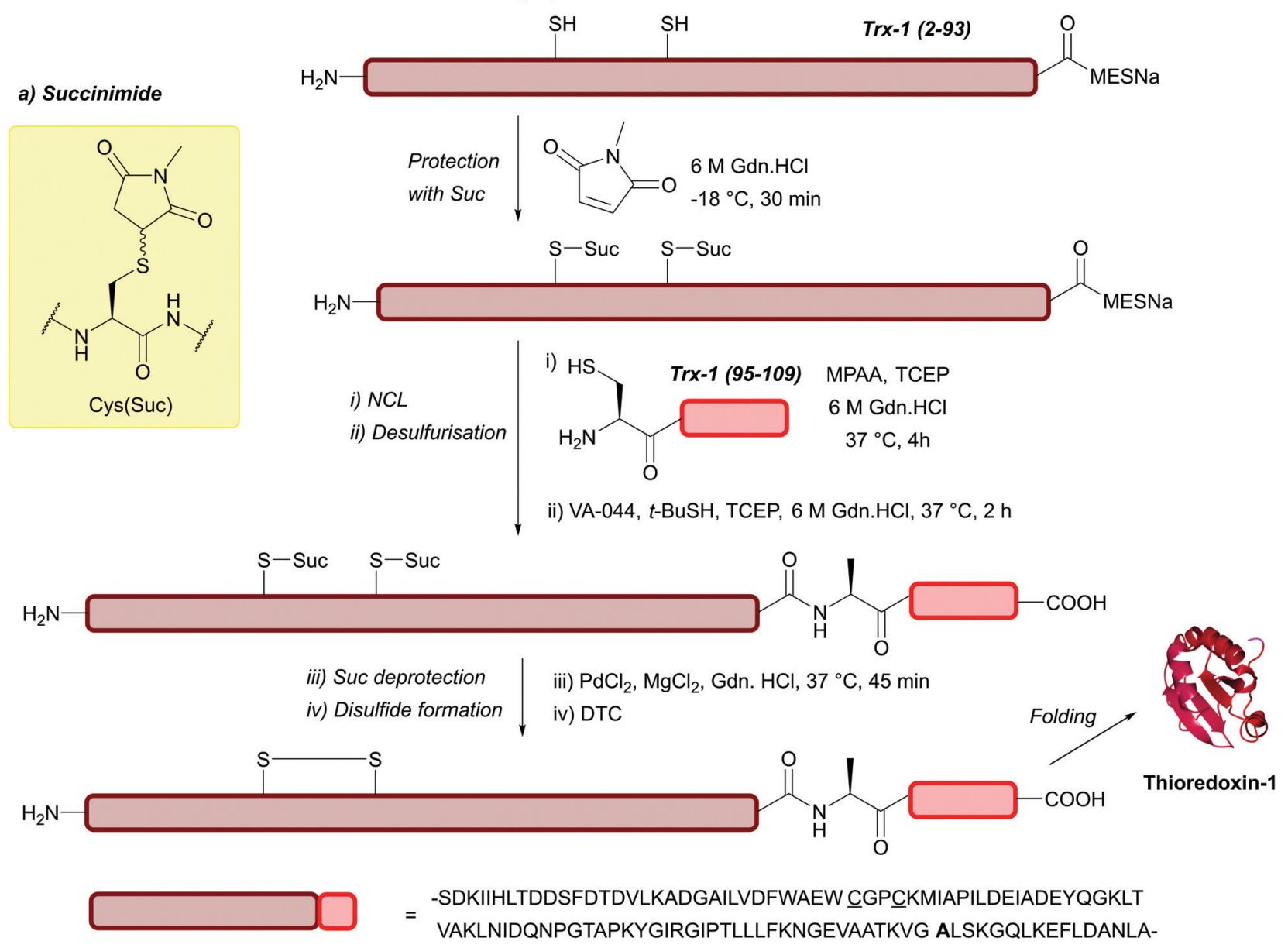

Fig. 29 (a) Cys thiol protection with $N$-methyl maleimide yielding a thiol-succinimide conjugate (Suc). (b) Synthesis of thioredoxin-1 using Cys(Suc).

acids. ${ }^{137}$ The Thz group simultaneously protects the thiol and the amino group of Cys (Fig. 30a), and can be removed using oxidants such as $\mathrm{H}_{2} \mathrm{O}_{2}$ and $\mathrm{I}_{2}$. Removal will also occur following treatment with iodoacetic acid and benzyl chloride ( $\mathrm{pH} \mathrm{10-11,}$ $\mathrm{RT}$ ), or ferric chloride in air ( $\mathrm{pH}$ 10). ${ }^{137}$ Alternatively, deprotection of Thz can be achieved by adjusting the reaction mixture to $c a$. pH 4 in the presence of a large excess of methoxyamine. Due to the ease of deprotection using this strategy, the Thz protecting group has seen widespread use in chemical protein synthesis, with one of the first examples being the total chemical synthesis of Crambin reported in 2004 (Fig. 30b). ${ }^{39}$ The group has also been employed in the synthesis of other proteins such as ubiquitinated proteins, ${ }^{232}$ histones, ${ }^{233}$ and HIV-1 protease. ${ }^{234}$ The use of methoxyamine to deprotect Thz has proven, however, to be incompatible with one-pot ligations involving thioesters (undesired reaction at/hydrolysis of the thioester group). ${ }^{233}$ Thz has also been shown to be unstable towards $\mathrm{NaNO}_{2}$ treatment at $\mathrm{pH} 3-4$ during activation of peptide hydrazides; this issue can be circumvented by using a 2-(tert-butyldisulfanyl)ethyloxycarbonyl protected Thz group (Tbeoc$\mathrm{Thz}$ ) in place of Thz, which is stable to $\mathrm{NaNO}_{2}$ hydrazide activation, and can be converted to Thz upon reduction with TCEP. ${ }^{47}$

More recently, alternative strategies for the deprotection of Thz have been described; these have primarily been transition-metal based. ${ }^{235}$ For example, deprotection of Thz can be achieved using water-soluble $\mathrm{Pd}(\mathrm{II})$ complexes, such as $[\mathrm{Pd}(\text { allyl }) \mathrm{Cl}]_{2}$ followed by treatment with DTT to both obtain the free thiol and quench remaining Pd species (Fig. 30c). This is performed under native chemical ligation (NCL) conditions and in the presence of MPAA and TCEP, with complete removal obtained within 15 min. ${ }^{138}$ MPAA and TCEP appear to be crucial for efficient removal (100\% removal in $15 \mathrm{~min}$ vs. $40 \%$ removal after $4 \mathrm{~h}$ ). It has been hypothesised that this is due to MPAA and TCEP chelating to $\mathrm{Pd}(\mathrm{II})$, or possibly reducing it to $\mathrm{Pd}(0)$. To demonstrate the usage of this deprotection method, Lys34-ubiquitinated H2B and several other sample peptides have been synthesised using $\operatorname{Pd}(\mathrm{II})$ to deprotect Thz. ${ }^{138}$ In further work, it was found that $[\mathrm{Pd}(\text { allyl }) \mathrm{Cl}]_{2}$ and GSH (1:1) in $6 \mathrm{M} \mathrm{Gdn} \cdot \mathrm{HCl}\left(\mathrm{pH} 6.5,37{ }^{\circ} \mathrm{C}, 45 \mathrm{~min}\right)$ were sufficient for full removal, along with its orthogonality demonstrated to both Acm and $t \mathrm{Bu} .{ }^{85} \mathrm{Pd}$-mediated Thz deprotection has also been successfully applied towards in vivo systems for triggered release/chemical activation of peptides/proteins. ${ }^{236}$ Furthermore, Thz analogues have also been employed in within the field of protein bioconjugation; Pd-mediated ${ }^{237}$ (or Ag-mediated) ${ }^{238}$ "unmasking” of unnatural Thz side chains leads to generation of an $\alpha$-oxo aldehyde side chain, which can be used for downstream site-selective protein modification. ${ }^{239}$ Aside from 
a) Thiazolidine

b) Synthesis of crambin<smiles>CC(C)NC(=O)[C@H]1CSCN1</smiles><smiles>[R]C(=O)CCCC(=O)C1CSCN1</smiles>

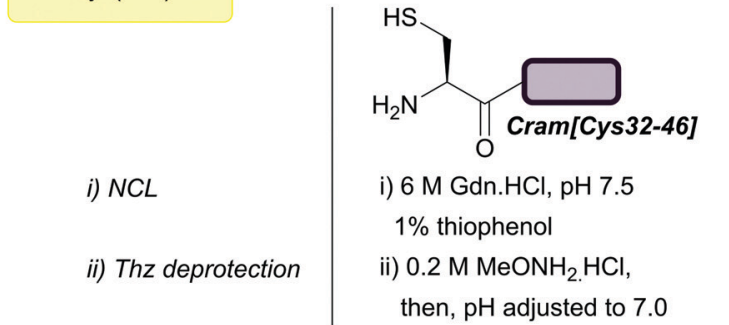<smiles>N[C@@H](CS)C(=O)CCCC(=O)N[C@@H](CS)C(=O)CCCCC(=O)O[Mg]</smiles>

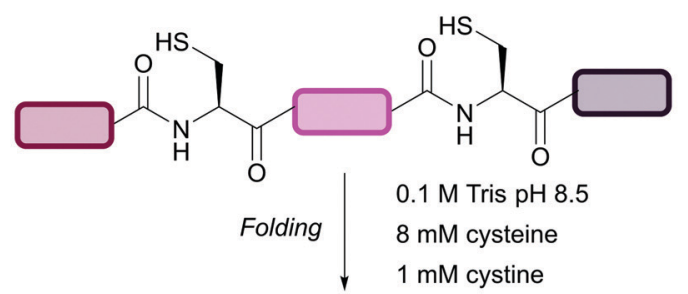

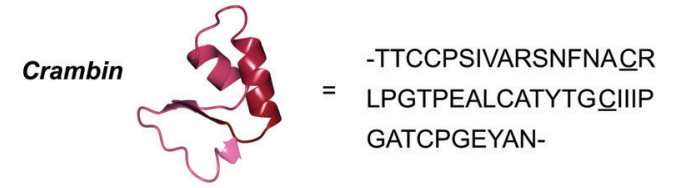

c) Palladium-mediated deprotection

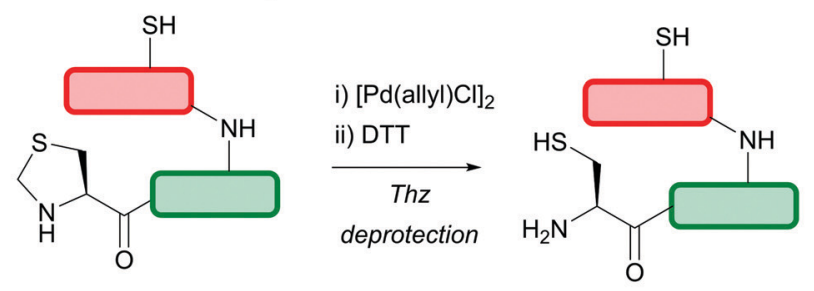

\section{= $-M L I K V K T L T G K E I E I D I E P T D K V E R I K E R V E E K E G I P P Q Q Q R L I$} YSGKQMNDEKTACDPYILGGSVLHLVLALRGG-

$=-\underline{C}$ IVRIMKMRKVLKHQ-

\section{d) Copper-mediated deprotection}<smiles>[R]C(=O)C1CCCC1</smiles>

i) Thz deprotection ii) $\mathrm{NCL}$

i) $\mathrm{CuSO}_{4}$, ascorbate, then DTT, purification ii) $6 \mathrm{M}$ Gdn. $\mathrm{HCl}, 0.1 \mathrm{M}$ HEPPS 40 mM MPAA, 30 mM TCEP, pH 7.0<smiles>CCCCCCC(=O)N[C@@H](CS)C(=O)C1CCCC1</smiles>
Folding $\downarrow \begin{aligned} & 3 \mathrm{M} \mathrm{Gdn} . \mathrm{HCl} \\ & 0.1 \mathrm{M} \text { phosphate buffer } \mathrm{pH} 7.7\end{aligned}$ CXCL14 $=\begin{aligned} & \text {-SKCKCSRKGPKIRYSDVKKLEMKPKY } \\ & \text { PLCEEKMMVIITTKSVSRYRGQEHCLLHP }\end{aligned}$<smiles>CCNC(=O)[C@@H]1CSC2(N1)C(=O)c1ccccc1C2=O</smiles>
f) Cyclic peptide synthesis

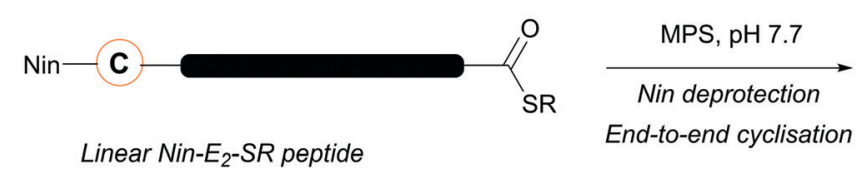

= -GKFTRSQKEGLHYTCSSHFPYSQYQFWKNFQTLKIVIKG-

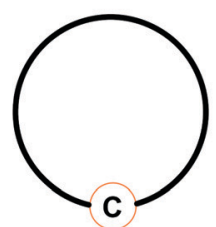

Cyclised peptide

Fig. 30 (a) N-terminal Cys protection with the thiazolidine (Thz) protecting group. (b) Synthesis of Crambin using Cys(Thz) with methoxyaminemediated deprotection. (c) Peptide synthesis using Cys(Thz) with Pd-mediated deprotection. (d) Synthesis of CXCL14 using Cys(Thz) with Cu-mediated deprotection. (e) N-terminal Cys protection with the ninhydrin (Nin) protecting group. (f) Cyclic peptide synthesis using Cys(Nin).

$\mathrm{Pd}, \mathrm{Cu}$ complexes can also be used to deprotect Thz. ${ }^{139,240} \mathrm{Thz}$ is removed by $\mathrm{CuSO}_{4}$ in the presence of sodium ascorbate (critical for avoiding oxidation by-products), in $5 \mathrm{M} \mathrm{Gdn} \cdot \mathrm{HCl}$ in HEPPS buffer ( $\mathrm{pH} 7.0,1 \mathrm{~h}, 37^{\circ} \mathrm{C}$ ). This was demonstrated in the synthesis of CXCL14 (Fig. 30d). ${ }^{139}$ As discussed previously, the reaction can then be quenched with DTT or EDTA. ${ }^{73}$ No epimerisation or side products are observed using this method of deprotection, and the reaction can be performed under standard NCL conditions. ${ }^{139}$ Outside of transition-metal based strategies, 2,2 -dipyridyl disulfide (DPDS) in $50 \% \mathrm{MeCN}(0.1 \% \mathrm{TFA})$ as a reagent for Thz deprotection has also been described. ${ }^{140,241}$

5.8.2 Ninhydrin (Nin). The 1,2,3-indanetrione monohydrate (ninhydrin, Nin) group can be used to modify N-terminal Cys residues, protecting both the amine and thiol moiety as a 
thiazolidine ring (Fig. 30e). ${ }^{242}$ Nin has since been used as an Nterminal Cys protecting group used in Boc SPPS. ${ }^{141}$ The Nin group can be converted back to the Cys in the presence of an excess of Cys (pH 7.7, 30 min, $23{ }^{\circ} \mathrm{C}$ ). Cysteine $O$-methylester in DMF/DIEA will remove the group while the peptide is still attached to the resin. However, using Cys deprotection is not possible for thioester-containing peptides as Cys can form amide bonds with thioesters. Deprotection can be achieved in this case using excess 3-mercaptopropiosulfonic acid (MPS) at pH 7.7 (which is nearly as effective as Cys-mediated deprotection) or under reducing conditions $\left(10 \% \mathrm{TFA} / \mathrm{H}_{2} \mathrm{O} / \mathrm{Zn}\right.$ dust, $\left.1 \mathrm{~h}\right){ }^{141}$

Protection using the Nin group avoids the formylation side reactions normally found during TFA and HF cleavage when His(Bom) groups are present, without the need for additional scavengers. Additionally, in thioester-containing peptides, the products will cyclise under deprotection conditions, enabling a one-step process. As proof of concept, a Nin- $\mathrm{E}_{2}-\mathrm{SR}$ peptide was synthesised and cyclised in one-step (Fig. 30f), using an excess of MPS (where $E_{2}$ refers to a 40-residue long sequence of the chemokine receptor CCR5's second extracellular loop). ${ }^{141}$

\subsection{Photo-labile protecting groups}

5.9.1 2-Nitrobenzyl (oNB). 2-Nitrobenzyl (oNB) is a photocleavable Cys protecting group (Fig. 31a) first described in $1981,{ }^{243}$ and can be removed by irradiation at $\geq 350 \mathrm{~nm} .^{142}$ The protecting group is compatible with Boc and Fmoc SPPS, and can be cleaved under aqueous conditions; for example, in deoxygenated MeCN/0.05 M PBS, 1:1 at pH 6, in the presence of semicarbazide and $\mathrm{L}_{-}(-)$-ascorbic acid. ${ }^{143}$ The $o$ NB protecting group also shows a high one-photon efficiency, and a high yield of the free thiol is obtained upon photolysis. ${ }^{145}$

The protecting group has since featured in the synthesis of a thioester-containing HCDLP pentapeptide; this peptide can then undergo NCL to semisynthesise a variant of a nickeldependant superoxide dismutase (NiSOD). Critically, Cys $(o \mathrm{NB})$ remained intact after acidic deprotection of other amino acid residues (95\% TFA) of the pentapeptide, and subsequent NCL to a recombinant Streptomyces coelicolor NiSOD bearing an $\mathrm{N}$-terminal Cys. Photochemical deprotection of $o \mathrm{NB}$ could then be achieved by irradiation at $365 \mathrm{~nm}$ (100 mM NaOAc, $20 \mathrm{mM}$ TCEP, $10 \mathrm{mM}$ semicarbazide, $\mathrm{pH} 5.8) .{ }^{244} \mathrm{Cys}(\mathrm{oNB})$ can also be
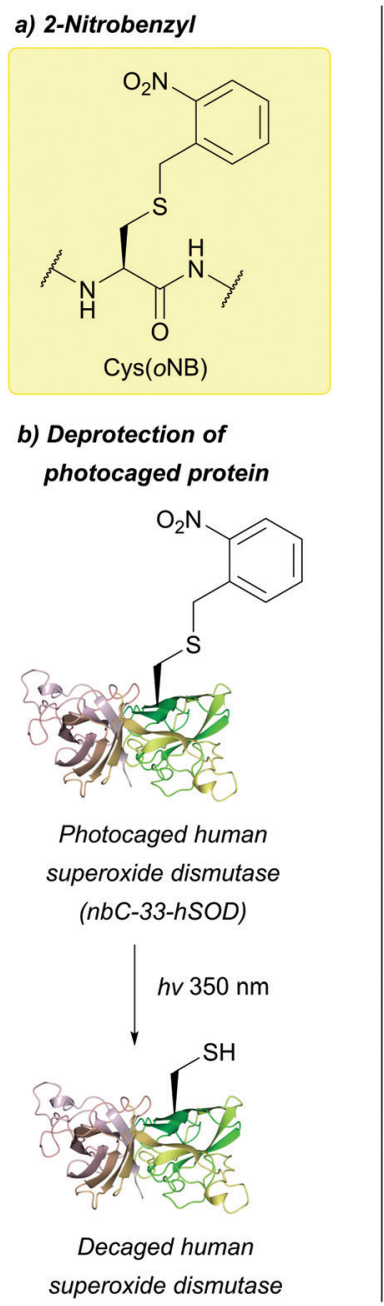

c) Partial synthesis of plectasin

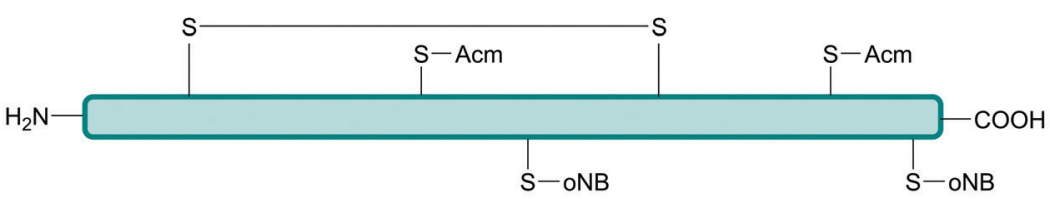

\begin{tabular}{l|l} 
oNV deprotection & $h v 350 \mathrm{~nm}$ \\
Disulfide formation & $\quad 6 \mathrm{M} \mathrm{Gdn} . \mathrm{HCl}, \mathrm{pH} 7,25^{\circ} \mathrm{C}$
\end{tabular}

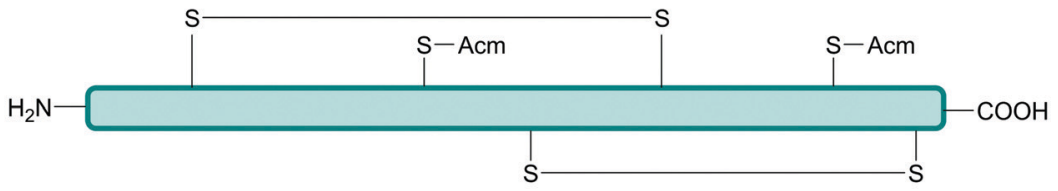

\begin{tabular}{l|l} 
i) Acm deprotection & i) $\mathrm{PdCl}_{2}, \mathrm{pH} 1$ \\
$\begin{array}{ll}\text { ii) } P d \text { quench } \\
\text { iii) Disulfide formation }\end{array}$ & ii) $\mathrm{DTC}$ \\
iii) DSF, $6 \mathrm{M} \mathrm{Gdn} \cdot \mathrm{HCl}, \mathrm{pH} 7,37^{\circ} \mathrm{C}$
\end{tabular}
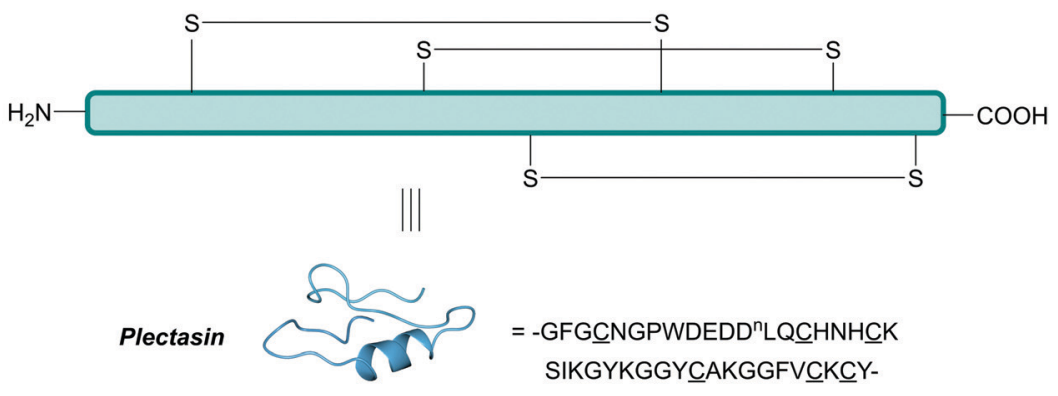

Fig. 31 (a) Cys thiol protection with the 2-nitrobenzyl (oNB) protecting group. (b) Photodecaging of a photocaged human superoxide dismutase containing Cys(oNB) as an unnatural amino acid (nbC-33-hSOD). (c) Partial synthesis of plectasin using Cys(oNB). 
incorporated into proteins via unnatural amino acid mutagenesis $^{245,246}$ as demonstrated with a photocaged human superoxide dismutase (nbC-33-hSOD, Fig. 31b), ${ }^{246}$ along with being used to study protein activity, ${ }^{247,248}$ As discussed previously, $\operatorname{Cys}(o \mathrm{NB})$ is also orthogonal to Pd-mediated deprotection of Acm, as recently displayed in the synthesis of Linaclotide and plectasin (Fig. 31c). ${ }^{215}$ The $o$ NB protecting group is, however, a poor chromophore and displays low two-photon sensitivity. Coumarin-based protecting groups have since been described in an attempt to address some of these issues. ${ }^{145}$

5.9.2 [7,8-Bis(carboxymethoxy)coumarin-4-yl]methoxycarbonyl (7,8-BCMCMOC), [7-bis(carboxymethyl)-amino-coumarin-4-yl]methoxycarbonyl (BCMACMOC), and $\alpha$-carboxy-methoxy-2-nitrobenzyl (C4MNB). [7,8-Bis(carboxymethoxy)coumarin-4-yl]methoxycarbonyl (7,8-BCMCMOC, Fig. 32a), [7-bis(carboxymethyl)-aminocoumarin-4-yl]methoxycarbonyl (BCMACMOC, Fig. 32b), and $\alpha$-carboxy-4-methoxy-2-nitrobenzyl (C4MNB, Fig. 32c) were reported for use as $\mathrm{H}_{2} \mathrm{O}$ soluble, photocleavable Cys protecting groups in 2009. ${ }^{144}$ The 7,8-BCMCMOC and BCMACMOC groups are based on (coumarin-4-yl)methyl chromophores, whereas the C4MNB group is based on a 2-nitrobenzyl chromophore (along with a $\alpha$-carboxy-4,5-dimethoxy-2-nitrobenzyl (CDMNB) group that was also reported). All groups were reported to show stability towards TFA and thiolysis, and all three protectedFmoc-Cys derivatives showed good solubility in HEPES buffer: MeCN (95:5, pH 7.2). The 7,8-BCMCMOC and BCMACMOC groups were, however, unstable when treated with piperidine, resulting in intramolecular $S$ - $>N$ acyl shifts in the case of $\mathrm{N}$-terminal Cys; an alternative Fmoc removal protocol (1\% DBU in DMF, acidic washing) and acetylation of the $\mathrm{N}$-terminus was therefore utilised. The BCMACMOC group can be removed by irradiation at $\geq 402 \mathrm{~nm}$ whilst the 7,8 -BCMCMOC and C4MNB groups can be removed by irradiation at $\geq 325 \mathrm{~nm}$, allowing for orthogonal deprotection (provided BCMACMOC is photolysed using long-wavelength irradiation first). These groups were subsequently used to synthesise an $\mathrm{N}$-acetylated resact peptide (Ac ${ }^{0}$-resact). ${ }^{144}$

5.9.3 2-Nitroveratryl (oNV). Originally described as a photolabile group for other functional groups, ${ }^{249-251}$ the 2-nitroveratryl/ 6-nitroveratryl (oNV), or 4,5-dimethoxy-2-nitrobenzyl (DMNB) group was first introduced as a Cys protecting group (Fig. 33a) for use in Fmoc SPPS in 2014. ${ }^{142}$ Removal of this group was achieved by irradiation at $350 \mathrm{~nm}$ for $30 \mathrm{~min}$ in aqueous media without the need for additional reagents, and displayed a stronger molar absorptivity at $350 \mathrm{~nm}$ than $o \mathrm{NB}$. No significant racemisation $(<0.5 \%)$ was observed upon the incorporation of Cys(oNV) during SPPS using diisopropylcarbodiimide (DIC) and HOBt activation. ${ }^{142}$

$o \mathrm{NV}$ is fully compatible with Fmoc SPPS, including stability to $10 \%$ TFMSA/TFA in the presence of excess dipyridine disulfide, the conditions necessary to convert the $t \mathrm{Bu}$ protecting group to the activated $S$-Pyr group. The two groups may, therefore, be used together - photolysis of the oNV is followed by thiolysis in the presence of Cys(S-Pyr), selectively forming a disulfide bond. This strategy has been used for the solid-phase synthesis of several Cys-rich peptides, including human insulin and $\alpha$-conotoxin a) [7,8-Bis(carboxymethoxy)coumarin-4-yl]methoxycarbonyl
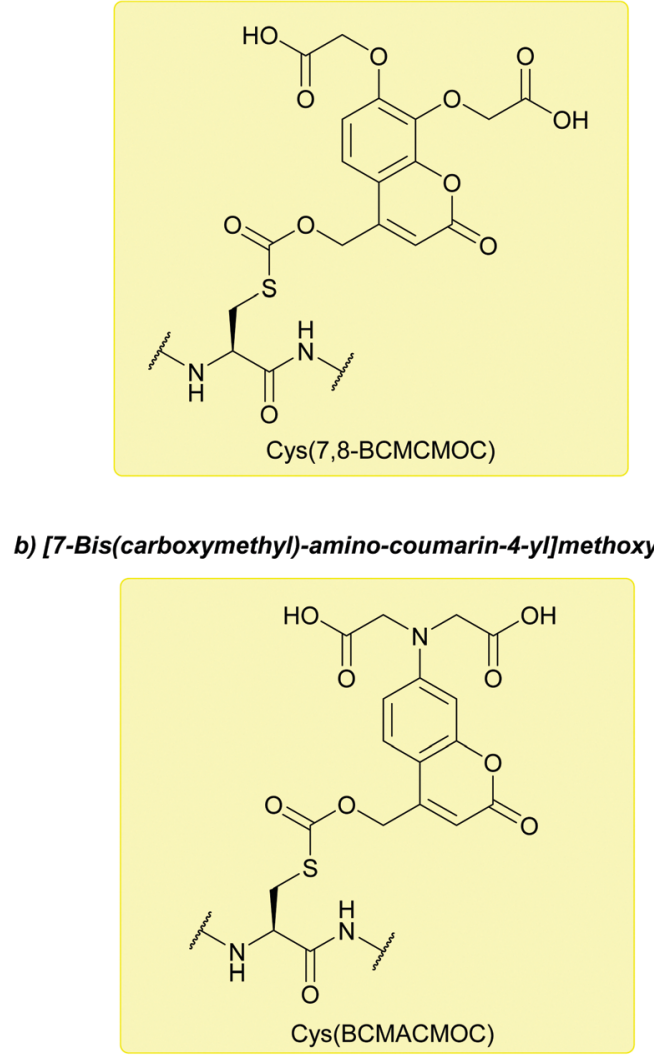

c) $\alpha$-Carboxy-4-methoxy-2-nitrobenzyl

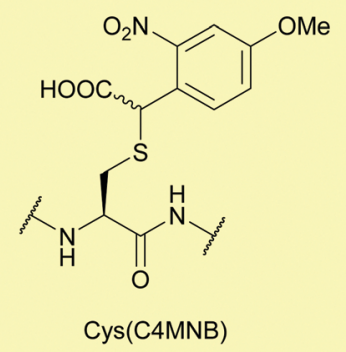

Fig. 32 (a) Cys thiol protection with the [7,8-bis(carboxymethoxy)coumarin-4-yllmethoxycarbonyl (7,8-BCMCMOC) protecting group. (b) Cys thiol protection with the [7-bis(carboxymethyl)-amino-coumarin-4-yl]methoxycarbonyl (BCMACMOC) protecting group. (c) Cys thiol protection with the $\alpha$-carboxy-4-methoxy-2-nitrobenzyl (C4MNB) protecting group.

ImI (Fig. 33b). ${ }^{142}$ oNv photocaged Cys has since been utilised in the synthesis of multi-Cys-containing peptide fragments. ${ }^{252}$ The protected amino acid has also been incorporated into proteins via unnatural amino acid mutagenesis to yield photocaged eGFP (EGFPTyr39DMNB-Cys), ${ }^{253}$ and photocaged glutathione peroxidase 3 (caged Gpx3 Cys32); the latter can then be oxidised with $\mathrm{H}_{2} \mathrm{O}_{2}$ to generate the sulfenic acid analogue (caged Gpx3 Cys32O, Fig. 33c). ${ }^{254}$

5.9.4 6-Bromo-7-hydroxycoumarin (Bhc). 6-Bromo-7-hydroxycoumarin (Bhc) was first described as a thiol-protecting group in $2008,{ }^{255}$ and described as a Cys protecting group (Fig. 34a) 
a) 2-Nitroveratryl

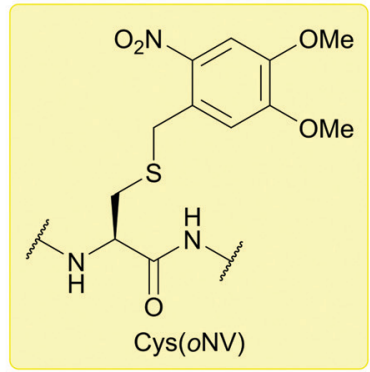

b) Partial synthesis of $\alpha$-conotoxin ImI

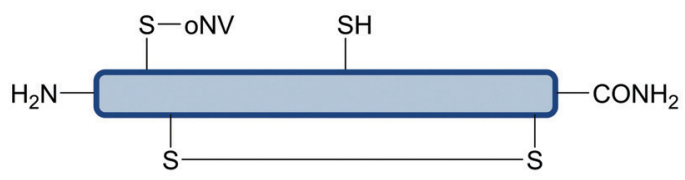

oNV deprotection $\mid h v 350 \mathrm{~nm}$

Disulfide formation $1: 1 \mathrm{H}_{2} \mathrm{O}: \mathrm{MeCN}, 1 \% \mathrm{AcOH}$

$\checkmark \mathrm{pH} 5.5$

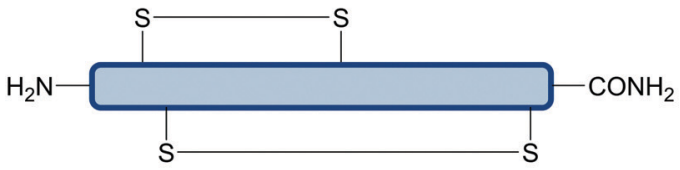

$\alpha$-Conotoxin ImI

$=-$ GCCSDPRCAWRC- c) Cys(oNV) oxidation
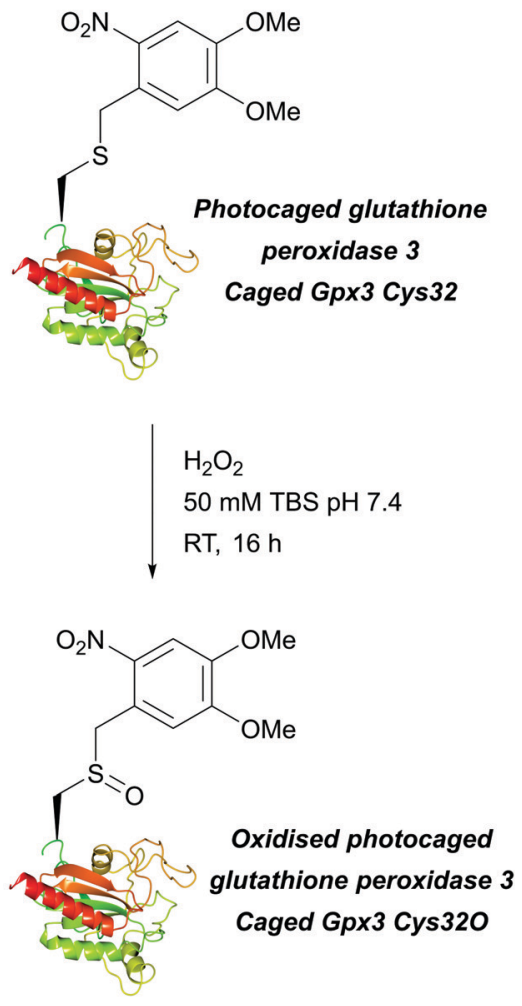

Fig. 33 (a) Cys thiol protection with the 2-nitroveratryl (oNV) protecting group. (b) Partial synthesis of $\alpha$-conotoxin Iml using Cys(oNV). (c) Photocaged glutathione peroxidase 3 (caged Gpx3 Cys32) and subsequent oxidation to yield the the sulfenic acid analogue (caged Gpx3 Cys32O).

in 2016. ${ }^{145}$ Irradiation at $365 \mathrm{~nm}$ in buffer $(1 \mathrm{mM}$ DTT in $50 \mathrm{mM}$ $\mathrm{PB}, \mathrm{pH}$ 7.2) causes deprotection. Unlike $o \mathrm{NB}$, Bhc has a high twophoton sensitivity; for two-photon excitation, wavelengths of $800 \mathrm{~nm}$ may be used.

Although incorporation into a peptide using Fmoc SPPS is not an issue, the photocleavage efficiency of Bhc-protected thiols is context-dependent. This is because the major product of irradiation is typically an unwanted photoisomer (a 4-methylcoumarin-3-yl thioether, Fig. 34b) instead of the free thiol, which limits the applications of Bhc as a Cys protecting group.

5.9.5 Nitrodibenzofuran (NDBF). Nitrodibenzofuran (NDBF) was proposed as an alternative Cys protecting group (Fig. 34c) to Bhc in 2016. ${ }^{145}$ Like Bhc, NDBF is removed from Cys via irradiation at $365 \mathrm{~nm}$; two-photon deprotection is also possible at $800 \mathrm{~nm}$. The two-photon cross-section of NDBF is comparable to that of Bhcprotected acetate. Additionally, NDBF deprotection results in clean conversion to the free thiol without the occurrence of $S$-to- $N$ shifts, and displays a higher uncaging efficiency than that of oNV. ${ }^{145} \mathrm{NDBF}$ was also found to be compatible with Fmoc SPPS, allowing for the synthesis of Cys(NDBF) photocaged K-Ras-derived peptides. These photocaged peptides could then be decaged with irradiation at $800 \mathrm{~nm}$, and the free thiol enzymatically farnesylated in situ in the presence of protein farnesyltransferases (PFTase) and farnesyl diphosphate; as anticipated, farnesylation did not occur if the Cys residue was protected with NDBF.
The NDBF protecting group has been additionally used for live cell applications. A fluorescent, farnesylated peptide which can undergo enzymatic palmitoylation by palmitoyl acyltransferase was synthesised and protected as a NDBF thioether (Fig. 34d). ${ }^{145}$ Incubation of this peptide with human ovarian carcinoma SKOV3 cells led to localisation of the peptide to the cytosol and the Golgi apparatus. Upon irradiation, the peptide migrated to the plasma membrane, indicating that enzymatic palmitoylation had occurred (Fig. 34e).

5.9.6 6-Bromo-7-hydroxy-3-methylcoumarin (mBhc). The 6-bromo-7-hydroxy-3-methylcoumarin (mBhc) protecting group (Fig. 35a) is an analogue of the Bhc protecting group. ${ }^{146}$ Compared to Bhc, the $\mathrm{C} 3$ position of mBhc is alkylated, which prevents photoisomerisation upon irradiation allowing for decaging to occur. Photolysis can be achieved using with one or two-photon excitation. Controlled prenylation of K-ras derived peptides was also demonstrated in a manner similar to that described for the Cys(NDBF) group. Furthermore, mBhc caged thiols could be used to design 3D patterns in hydrogel matrices. ${ }^{146}$

5.9.7 Methoxy-nitrodibenzofuran (OMe-NDBF). Very recently, methoxy-nitrodibenzofuran (OMe-NDBF) has been suggested as an alternative to NDBF for the protection of the Cys thiol (Fig. 35b) with a higher two-photon photolysis efficiency. ${ }^{147}$ Additionally, in-depth analysis into suppressing Cys racemisation in the coupling of both Fmoc-Cys(NDBF) and Fmoc-Cys(OMe-NDBF) was performed; 
a) 6-Bromo-7-hydroxycoumarin<smiles>CC(C)CNC(=O)[C@H](CSCc1cc(=O)oc2cc(O)c(Br)cc12)NC(C)C</smiles>

b) Photoisomerisation of Cys(Bhc)

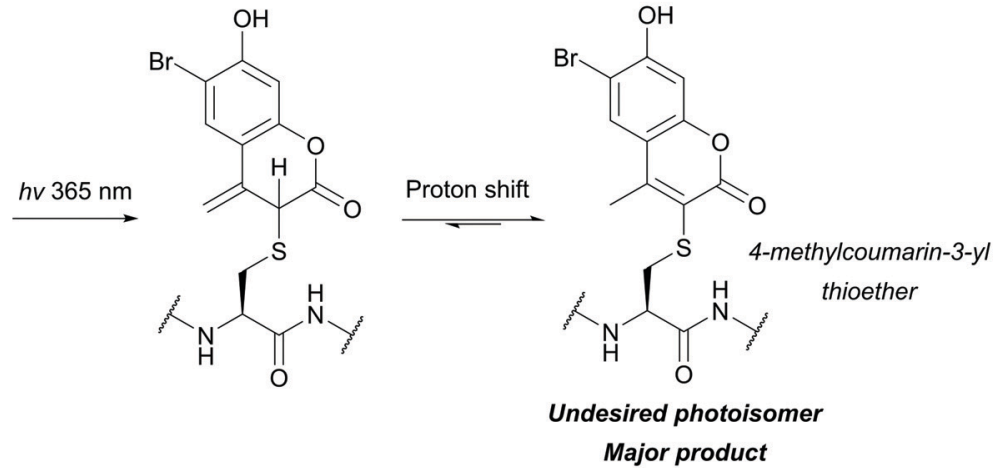

c) Nitrodibenzofuran

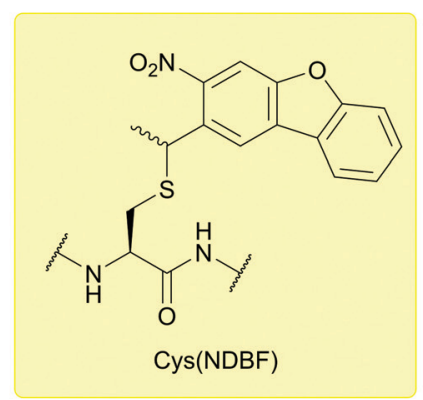

d) Cys(NDBF)-containing fluorescent, farnesylated peptide<smiles>CCCCNc1ccc([N+](=O)[O-])c2nonc12</smiles>

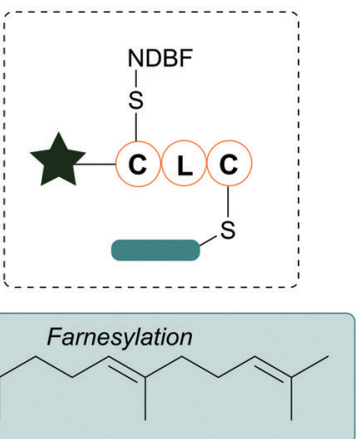

e) Cys(NDBF) deprotection in vivo

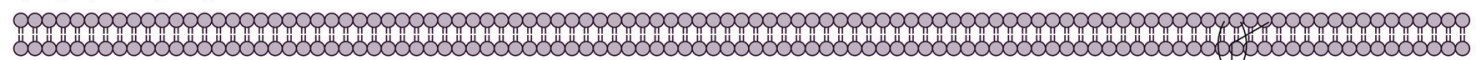
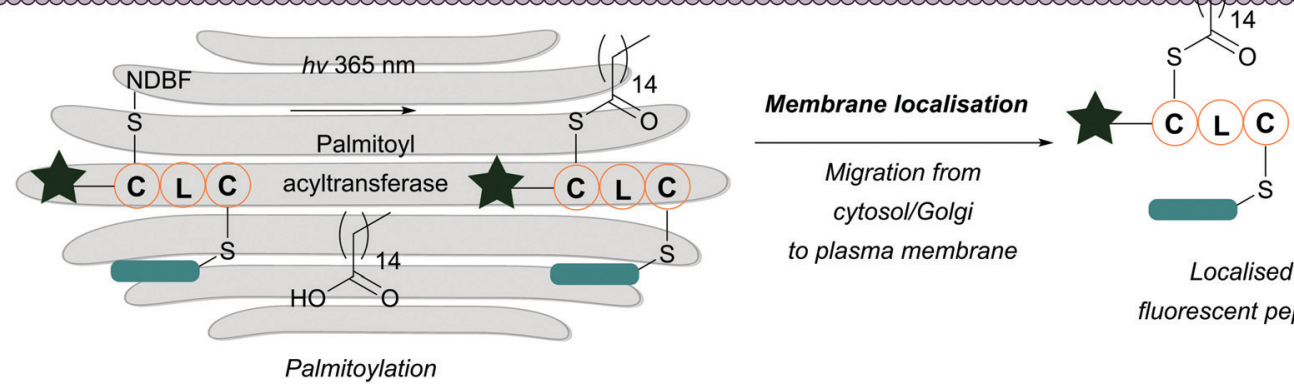

to plasma membrane

Localised

fluorescent peptide

Palmitoylation

Fig. 34 (a) Cys thiol protection with the 6-bromo-7-hydroxycoumarin (Bhc) protecting group. (b) Irradiation of Cys(Bhc) and subsequent formation of a 4-methylcoumarin-3-yl thioether photoisomer. (c) Cys thiol protection with the nitrodibenzofuran (NDBF) protecting group. (d) Fluorescent, farnesylated peptide containing Cys(NDBF). (e) Incubation and subsequent localisation in vivo of the aforementioned peptide within the cytosol and Golgi apparatus. Irradiation of the cells leads to deprotection of $\mathrm{Cys}(\mathrm{NDBF})$ which can then under enzymatic palmitoylation, leading to membrane localisation of the peptide.

employing benzotriazole-1-yl-oxy-tris-pyrrolidino-phosphonium hexafluorophosphate/1-hydroxy-7-azabenzotriazole (PyBOP/HOAT) coupling (using DIEA as a base) lead to $<2 \%$ racemisation observed when either of these were used in Fmoc SPPS. Irradiation of $\alpha$-factor-derived peptides containing Cys(OMe$\mathrm{NDBF})$ at $350 \mathrm{~nm}$ for less than $30 \mathrm{sec}$ proved sufficient for deprotection. As for Cys(NDBF), Cys(OMe-NDBF) caging and decaging can be used to control farnesylation of photocaged K-ras derived peptides. ${ }^{147}$

\subsection{Reducing agent-labile protecting groups}

5.10.1 para-Nitrobenzyl ( $p \mathrm{NB})$. The use of the para-nitrobenzyl $(p \mathrm{NB})$ group for protecting Cys (Fig. 36) was first proposed in $1957 .{ }^{256} p$ NB was originally removed by catalytic hydrogenation in the presence of $10 \%$ palladium on carbon $(3 \mathrm{~h}, \mathrm{RT}$, atmospheric pressure). ${ }^{256}$ However, it was later reported that $\mathrm{H}_{2} / \mathrm{Pd} / \mathrm{C}$ reduction of pNB can lead to para-aminobenzyl ( $p \mathrm{AB})$ formation instead. ${ }^{257}$ To avoid this, the group can be removed by a reducing agent, followed by an oxidising agent to remove $p \mathrm{AB}$ and form a disulfide. $\mathrm{Zn} / \mathrm{AcOH}$ followed by $\mathrm{I}_{2}$ has been reported to be the most effective agent in solution, while $\mathrm{SnCl}_{2} / \mathrm{HCl}$ followed by $\mathrm{I}_{2}$ is the preferred agent for cleavage on solid support. On-resin, $\mathrm{I}_{2}$ oxidation resulted in complex mixtures and low yields. Other methods are less effective but still viable; oxidative removal of $p \mathrm{AB}$ can be achieved using an excess of ceric ammonium nitrate (CAN) and Hopkins reagent $\left(10 \% \mathrm{HgSO}_{4} / 5 \% \mathrm{H}_{2} \mathrm{SO}_{4}\right)$ but $\mathrm{I}_{2}$ in $\mathrm{AcOH}$ gives both a faster 
a) 6-Bromo-7-hydroxy-3-methylcoumarin<smiles>Cc1c(CSC[C@H](NC(C)C)C(=O)NC(C)C)c2cc(Br)c(O)cc2oc1=O</smiles>

b) Methoxy-nitrodibenzofuran

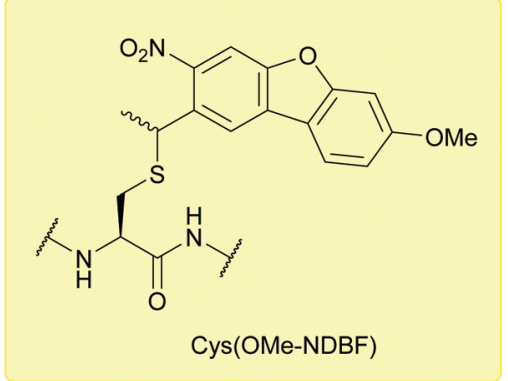

Fig. 35 (a) Cys thiol protection with the 6-bromo-7-hydroxy-3-methylcoumarin (mBhc) protecting group. (b) Cys thiol protection with the methoxy-nitrodibenzofuran (OMe-NDBF) protecting group.

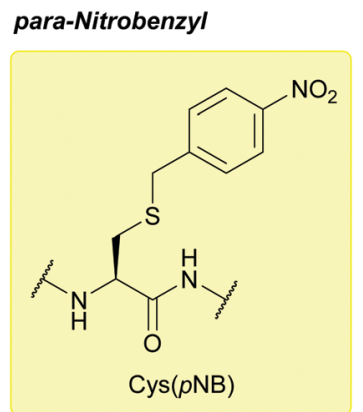

Fig. 36 Cys thiol protection with the para-nitrobenzyl ( $p N B$ ) protecting group.

and cleaner reaction in solution. ${ }^{111} p \mathrm{NB}$ is stable to $\mathrm{HF} / p$-cresol $\left(9: 1,1 \mathrm{~h}, 0{ }^{\circ} \mathrm{C}\right)$, TFA and $\mathrm{I}_{2} / \mathrm{AcOH} / 2 \mathrm{M} \mathrm{HCl}$. No side products are observed during cleavage. $p \mathrm{NB}$ has been proposed as an alternative to Acm for use in Boc SPPS due to its greater stability to acid. ${ }^{111}$

5.10.2 Carbomethoxysulfenyl (Scm). Carbomethoxysulfenyl (Scm) was first proposed in 1970, as a thiol protecting group (Fig. 37a) that could be used to form unsymmetrical disulfides. ${ }^{258} \mathrm{Scm}$ can be removed using thiols or other reducing agents, ${ }^{68}$ e.g. DTT. ${ }^{148} \mathrm{Scm}$ is stable to strong acids, including anhydrous HF and TFMSA. ${ }^{149}$ Cys(Acm) can be converted to Cys $(\mathrm{Scm})$ via treatment with methoxycarbonylsulfenyl chloride (Fig. 37b), where upon Cys(Scm) can be displaced by free thiols to form disulfides. ${ }^{148}$ However, there is some evidence that side reactions involving $S$-to- $N$ acyl migrations occur during coupling. The related derivative $\mathrm{Snm}$ has been recommended for use in Boc SPPS instead as side reactions are minimised. ${ }^{149}$ Both Cys(Scm) and Cys(Snm) have both been used in the synthesis of disulfide and trisulfide-containing oxytocin and deaminoxoxytocin. ${ }^{259}$

Cys(Scm) has recently used in protein bioconjugation to facilitate the modification of MB23-Cys (an Alphabody - a trihelical peptide with potential as an anti-cancer therapeutic) via disulfide linkages. MB23-Cys was first reduced using DTT (to remove any protein dimer), then modified with a folic acid, Cys(Scm)-containing peptide in $10 \mathrm{mM}$ Tris- $\mathrm{HCl}, \mathrm{pH} 7.4,37^{\circ} \mathrm{C}$ (Fig. 37c). ${ }^{148}$

5.10.3 ( $N^{\prime}$-Methyl- $N^{\prime}$-phenylcarbamoyl)sulfenyl (Snm). ( $N^{\prime}$-Methyl- $N^{\prime}$-phenylcarbamoyl)sulfenyl ( $\left.\mathrm{Snm}\right)$ is an Scm derivative synthesised in 1989 for Cys thiol protection. ${ }^{149}$ Snm can be removed under mild thiolytic conditions, e.g. DTT in the presence of $\mathrm{N}$-methylmorpholine (NMM) in $\mathrm{CDCl}_{3}$, which results in a mixture of the free thiol and the cyclic disulfide. If treated with 2-mercaptopyridine Snm is converted to the 2-pyridyl disulfide. Snm is stable to strong acids (anhydrous HF, TFMSA) and is less prone to undergoing side reactions than Scm - although a terminating side reaction is still observed when coupling of glycine is carried out in DMF. Snm is suitable for use in Boc SPPS. ${ }^{149}$ With regards to Fmoc SPPS, upon treatment with piperidine the Snm group is converted to a $S$-( $N$-piperidylcarbamoyl)sulfenyl group $(-\mathrm{S}(\mathrm{C}=\mathrm{O})$ Piperidine $)$, referred to as the Snip protecting group (Fig. 38a). These derivatives can be used for intramolecular disulfide formation both on and off resin akin to peptides containing Cys(Snm). ${ }^{259}$

5.10.4 4-Picolyl. The 4-picolyl protecting group (Fig. 38b) was originally removed by electrolytic reduction in a $0.5 \mathrm{M}$ sulfuric acid solution. ${ }^{151}$ However, it can be removed in a more convenient manner by $\mathrm{Zn} / \mathrm{AcOH}{ }^{150}$ The group is completely stable in TFA or $32 \% \mathrm{HBr}$ in AcOH (1 week, RT). ${ }^{151}$ However, the group is rarely used in SPPS. ${ }^{150}$

5.10.5 Sulfonic acid $\left(\mathrm{SO}_{3} \mathrm{H}\right)$ and sulfonyl $\left(\mathrm{SO}_{2} \mathrm{R}\right)$. The sulfonic acid $\left(\mathrm{SO}_{3} \mathrm{H}\right)$ group (Fig. 38c) is removed by thiols, ${ }^{152}$ e.g. DTT, BME, or phosphines, e.g. tributylphosphine. ${ }^{153}$ However, it is very acid sensitive and, as such, has little use in peptide synthesis. ${ }^{152}$ The sulfonate derivative $\left(\mathrm{SO}_{3} \mathrm{Na}\right)$ has, however, been shown to have sufficient stability to be used in both Boc and Fmoc SPPS. As proof of concept, $\mathrm{Arg}^{8}$-vasopressin was successfully synthesised using Cys $\left(\mathrm{SO}_{3} \mathrm{Na}\right)$ via Fmoc SPPS. Use of $p$-cresol as the sole scavenger during TFA cleavage from the resin presented premature removal of $\mathrm{SO}_{3} \mathrm{Na}$ from Cys. ${ }^{153}$

Sulfonyl $\left(\mathrm{SO}_{2} \mathrm{R}\right)$ protecting groups (Fig. 38d) have recently found some use in the synthesis of enantiomerically-enriched $\alpha$-hydroxy and $\alpha$-chloro acid building blocks. The sulfonyl group is first introduced to the Cys residue, and diazotisation is subsequently used to generate the $\alpha$-hydroxy or $\alpha$-chloro acid (depending on whether $\mathrm{H}_{2} \mathrm{SO}_{4}$ or $\mathrm{HCl}$ is used). ${ }^{260}$

5.10.6 3-Nitro-2-pyridinesulfenyl (Npys). The 3-nitro-2pyridinesulfenyl (Npys) ${ }^{261}$ protecting group (Fig. 38e) is a reducing agent-labile Cys protecting group. ${ }^{155}$ Npys can be removed in under $10 \mathrm{~min}$ using aliphatic thiols (e.g. 3-mercaptoacetic acid (MAA), BME). ${ }^{154}$ It can also be removed at room temperature using 
a) Carbomethoxysulfenyl<smiles>COC(=O)SSC[C@H](NC(C)C)C(=O)NC(C)C</smiles>

b) Conversion of Cys(Acm) to Cys(Scm)

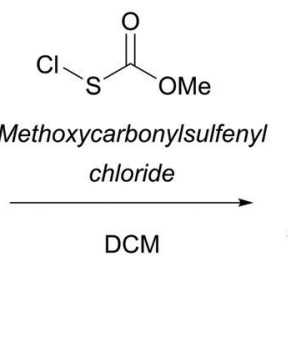<smiles>CCNC(CSSC(=O)OC)C(=O)NC(C)C</smiles>

c) Protein bioconjugation
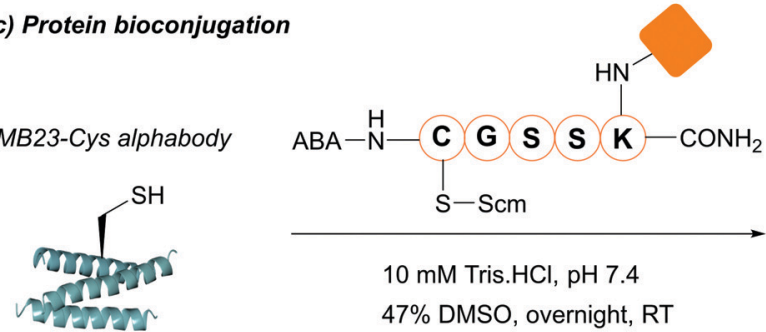

$10 \mathrm{mM}$ Tris. $\mathrm{HCl}, \mathrm{pH} 7.4$

$47 \%$ DMSO, overnight, RT

$=$ Folic acid

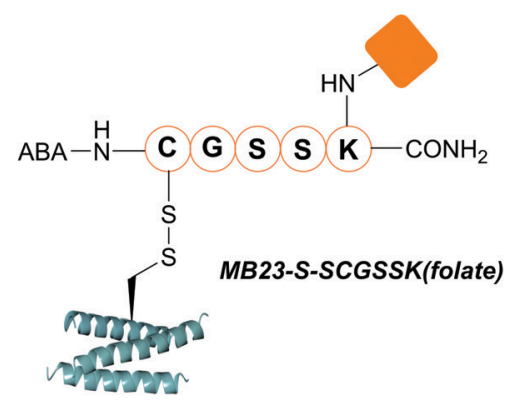

Fig. 37 (a) Cys thiol protection with the carbomethoxysulfenyl $(\mathrm{Scm})$ protecting group. (b) Conversion of Cys(Acm) to Cys(Scm) using methoxycarbonylsulfenyl chloride. (c) Protein bioconjugation with $\mathrm{Cys}(\mathrm{S} \mathrm{cm})$-containing peptides.

a) (N'-Methyl-N'-phenylcarbamoyl)sulfenyl and S-(N-piperidylcarbamoyl)sulfenyl

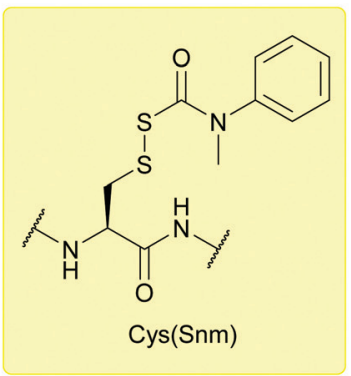

c) Sulfonic Acid

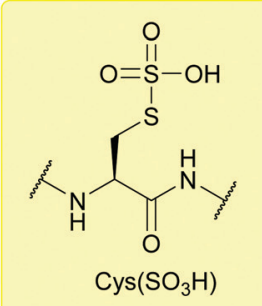

d) Sulfonyl

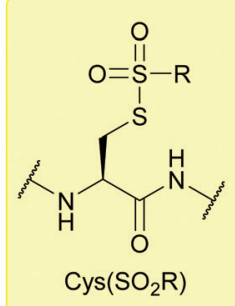

$20 \%$ piperidine in DMF

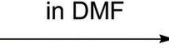

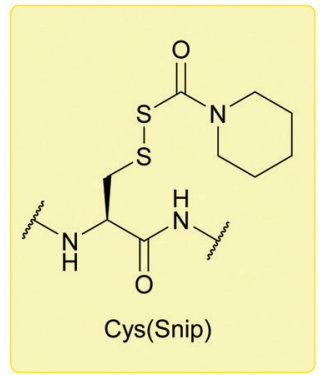

e) 3-Nitro-2-pyridinesulfenyl

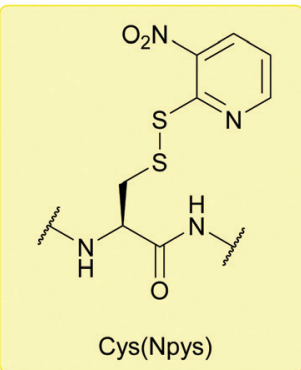

b) 4-Picolyl

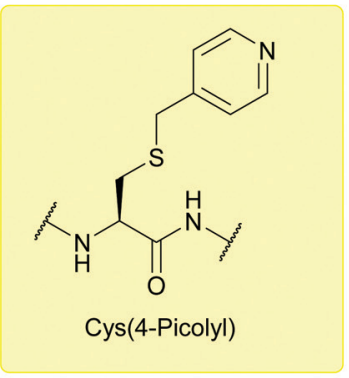

f) 5-Nitro-2-pyridinesulfenyl

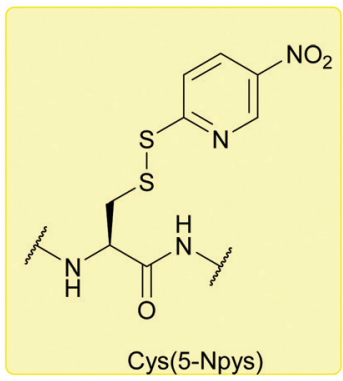

Fig. 38 (a) Cys thiol protection with the ( $N^{\prime}$-methyl- $N^{\prime}$-phenylcarbamoyl)sulfenyl (Snm) protecting group. Conversion of Cys(Snm) to Cys(Snip) occurs upon treatment with piperidine (b) Cys thiol protection with the 4-picolyl protecting group. (c) Cys thiol protection with the sulfonic acid ( $\left.\mathrm{SO}_{3} \mathrm{H}\right)$ protecting group. (d) Cys thiol protection with the sulfonyl $\left(\mathrm{SO}_{2} \mathrm{R}\right)$ protecting group. (e) Cys thiol protection with the 3-nitro-2-pyridinesulfenyl (Npys) protecting group. (f) Cys thiol protection with the 5-nitro-2-pyridinesulfenyl (5-Npys) protecting group.

tertiary phosphines in the presence of $\mathrm{H}_{2} \mathrm{O} .{ }^{155,156}$ Cys(Npys) is stable to strong acids such as TFA ( $24 \mathrm{~h}, \mathrm{RT}), \mathrm{HF}$ ( $1 \mathrm{~h}, \mathrm{RT})$ and $4 \mathrm{M}$ $\mathrm{HCl} /$ dioxane $\left(24 \mathrm{~h}\right.$ ) and is thus suitable for Boc SPPS. ${ }^{155}$ It is also stable to a wide variety of other reagents commonly used in Boc SPPS: DCM, DMF, $N, N$-dimethylacetamide, $N$-methylpyrrolidone, $\mathrm{MeOH}$, trifluoroethanol, pentafluorophenol. ${ }^{157} S$-Npys is somewhat 
stable towards aromatic thiols, which can cleave the $O$-Npys and $N$-Npys derivatives, enabling a degree of selectivity in its deprotection. ${ }^{154}$ It is relatively stable to the photolytic cleavage conditions used for peptide-(o-nitrobenzyl ester) resin bonds (350 nm). ${ }^{157}$ Npys can also act as an activating group for disulfide formation as it is displaced by the free thiol. ${ }^{155}$

Npys is not compatible with Fmoc SPPS, as it is unstable to piperidine (81\% decomposition following treatment with 50\% piperidine in DCM for $10 \mathrm{~min}$ ). It is also unstable to $\mathrm{TBAF}^{-}$, an alternative to piperidine for Fmoc deprotection. Additionally, while treatment with $\mathrm{HF}$ in the presence of anisole or $p$-cresol leaves Npys unaltered, HF in the presence of $p$-thiocresol or DMS (HF/DMS/p-cresol, $25: 65: 10$ or $\mathrm{HF} / \mathrm{DMS} / p$-cresol/ $p$-thiocresol, $25: 65: 5: 5)$, has been shown to cause significant loss of the protecting group. ${ }^{157}$ Npys has been used in many syntheses. ${ }^{154}$ An early example of this is the synthesis of [Lys $]^{8}$ vasopressin, which was chosen as a model peptide to display the use of Npys in Boc SPPS. ${ }^{156}$ More recently, the Npys protecting group has been the focus of a solid phase disulfide ligation (SPDSL) system. ${ }^{262}$ Here, Npys-Bn is loaded onto a solid support and converted to resinbound Npys-Cl through chlorosulfenylation. Cys $(t \mathrm{Bu})$-containing peptides can then be loaded onto the resin via Npys-mediated displacement of the $t \mathrm{Bu}$ protecting group. Peptide release, along with disulfide bond formation, can then be achieved through addition of a Cys-containing peptide. Subsequent intramolecular amide formation then yields a disulfide containing peptide, as demonstrated in the synthesis of oxytocin. ${ }^{262}$ A similar platform that replaces the Npys-Cl group for a more stable Npys-OPh(parafluoro) group has very recently been reported in the literature. ${ }^{263}$

5.10.7 5-Nitro-2-pyridinesulfenyl (5-Npys). 5-Nitro-2-pyridinesulfenyl (5-Npys, $p$-Npys, Fig. 38f) is an analogue of Npys. ${ }^{158} 5$-Npys retains almost all the properties of Npys and is also removed by thiols. ${ }^{158}$ It can also be partially removed (70-75\%) under mild conditions by a large excess of ascorbic acid in the presence of DTNP (pH 7, $35^{\circ} \mathrm{C}, 24$ h). ${ }^{264}$ Much like Npys, 5-Npys can be used as an activating group. The para-nitro group increases the acidity of the corresponding thiol and it is thus able to react at a lower $\mathrm{pH}$ than Npys. ${ }^{158}$ As with Npys, 5-Npys is incompatible with Fmoc SPPS. $^{90}$

5.10.8 tert-Butylsulphenyl (StBu). The tert-butylsulphenyl (StBu) Cys protecting group (Fig. 39a) has been described for use in both Boc ${ }^{265}$ and Fmoc $^{159}$ peptide synthesis. StBu can be removed under organic or aqueous conditions with reducing agents such as thiols, e.g. BME ${ }^{159}{ }^{\mathrm{DTT}},{ }^{266}$ or phosphines (e.g. $\mathrm{PBu}_{3},{ }^{267} \mathrm{PPh}_{3},{ }^{268} \mathrm{TCEP}^{269}$ ) The StBu protecting group is stable to acidic conditions (e.g. TFA:thioansiole:phenol, $95: 2.5: 2.5 \mathrm{v} / \mathrm{v}$ ) provided no thiol scavenger is added. In cases such as for tryptophan-containing peptides, 2-methylindole and anisole can be used as alternative scavengers to avoid StBu deprotection. ${ }^{159}$ The group is also compatible with basic conditions for Fmoc SPPS; extended incubations of resin-bound C-terminal Cys(StBu)containing peptides in $20 \%$ piperidine in DMF can lead to 3-(1-piperidinyl)alanine by-products however. ${ }^{77} \mathrm{StBu}$ can also be removed by TFA in the presence of DTNP-thioanisole. Interestingly, StBu exhibits no lability to TFA/DTNP without the addition of thioanisole to the mixture, making it orthogonal to groups which are removed by TFA/DTNP, such as $t \mathrm{Bu} .{ }^{80}$ Partial removal in HF has also been reported. ${ }^{159}$ The StBu protecting group is orthogonal to other protecting groups, such as Trt, Acm, Meb/Mob, $t \mathrm{Bu},{ }^{74,163}$ and Allocam. ${ }^{205}$ Protected Cys(StBu) has featured in the synthesis of somatostatin, ${ }^{268}$ coumarin-based probes, ${ }^{270}$ and cryptophane-based probes. ${ }^{271}$ Additionally, peptides bearing Cys(StBu) and perfluoroaryl-modified Cys residues can undergo cyclisation to form macrocyclic peptides; this was achieved with in situ reduction of Cys(StBu) with TCEP in the presence of glutathione $S$-transferase (GST, which facilitates conjugation of the newly released thiol and perfluoroaryl group, Fig. 39b). ${ }^{272}$ In the case of N-terminal Cys protected with StBu, in situ reduction/protecting group removal followed by NCL can be achieved in the presence of a suitable peptide thioester substrate. ${ }^{273,274}$ Alternatively, the N-terminal Cys can react with 2-cyano-6-aminobenzothiazoles (CBTs), as demonstrated in the synthesis of imaging agents for positron emission tomography (PET). ${ }^{269,275}$ For formation of disulfide bonds using an orthogonal protecting group strategy, StBu must be removed before other protecting groups to avoid cleaving or scrambling existing disulfides (as would occur upon treatment with a reducing agent). This is typically done on-resin prior to TFA acidolysis, which can result in side products and low yields. As discussed previously, one solution to this problem that has been reported was to perform post-synthetic tritylation; multiple disulfide bonds could then be regioselectively formed in solution using combinations of StBu, Trt, Acm and Meb/Mob, all of which survive the final cleavage step in Fmoc SPPS, bar Trt. ${ }^{74}$

Cys( $\mathrm{S} t \mathrm{Bu})$ has been used in the synthesis of a range of different peptides, including $\mu$-SIIIA (Fig. 39c), ${ }^{74} \alpha$-LvIA (Fig. 39d), ${ }^{205}$ and Linaclotide (Fig. 39e). ${ }^{276}$ The removal of StBu with reducing agents has previously been shown to be sequence-dependent and challenging; ${ }^{166}$ additionally, the lengthy times required for deprotection are undesirable when using the group in routine SPPS. ${ }^{163}$ Alternative disulfide-based protecting groups such as dimethoxyphenylthio (S-Dmp) and 2,4,6-trimethoxyphenylthio (S-Tmp) have since been reported as replacements for the StBu protecting group. $^{163}$

5.10.9 N-Methyl-phenacyloxycarbamidomethyl (Pocam). The $N$-methyl-phenacyloxycarbamidomethyl (Pocam) Cys protecting group (Fig. 40a) is both reducing agent- and acid-labile, removable by $\mathrm{Zn} / \mathrm{AcOH}$ (aq.) or TFA $\left(1 \mathrm{~h}, 50{ }^{\circ} \mathrm{C}\right)$. At lower temperatures, Pocam is relatively resistant to TFA treatment $\left(4 \mathrm{~h}, 4{ }^{\circ} \mathrm{C}\right)$. If Pocam is used for the thioester method of protein ligation it can be removed at the same time as $N$-azido groups protecting amino functionalities. Pocam has been used as an orthogonal protecting group in the synthesis of disulfide containing peptides, such as growth-blocking peptide (GBP) and $\alpha$-conotoxin SI. ${ }^{160}$ However, Pocam still has limited applicability due to its lack of acidstability and has since been effectively replaced by the introduction of Pac. ${ }^{6}$

5.10.10 Phenacyl (Pac). Introduced in 2008 for Boc solution phase synthesis, ${ }^{277}$ and in 2013 for Fmoc SPPS, ${ }^{161}$ the phenacyl (Pac) Cys protecting group (Fig. 40b) has effectively replaced the Pocam group. ${ }^{6}$ Pac may be removed using $\mathrm{Zn}$ in acid $\mathrm{AcOH}$ (aq.), MPS in $6 \mathrm{M} \mathrm{Gdn \cdot HCl).}{ }^{161,162}$ The group is slightly labile to 


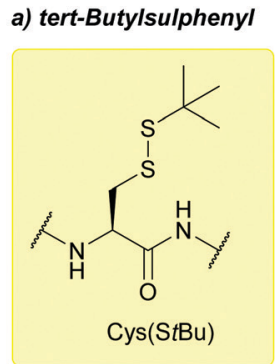

b) Peptide macrocylisation

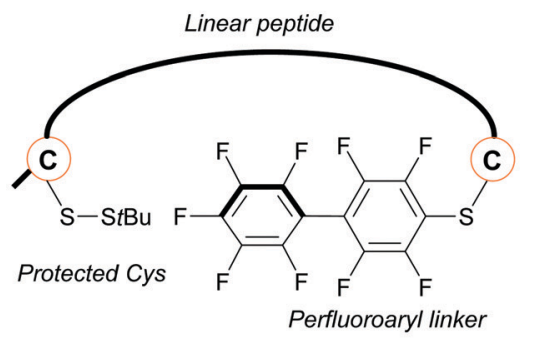

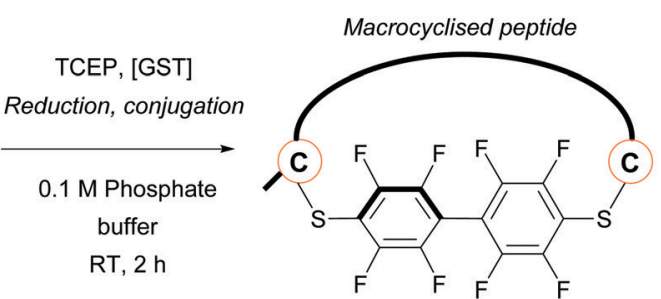

d) Partial synthesis of $\alpha 4 / 7$-Conotoxin LvIA ( $\alpha$-LvIA)

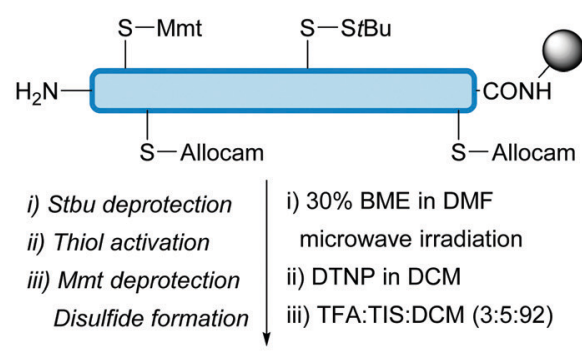

c) Partial synthesis of $\mu$-conotoxin SIIIA
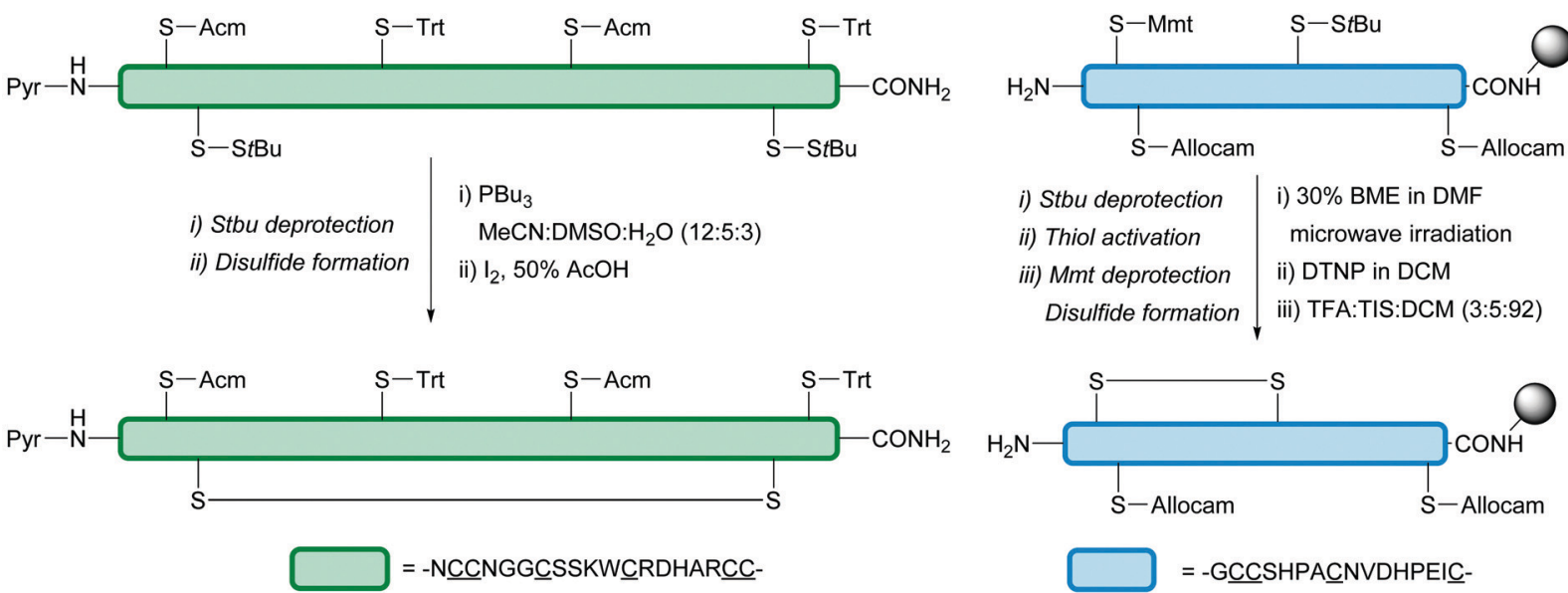

e) Linaclotide synthesis

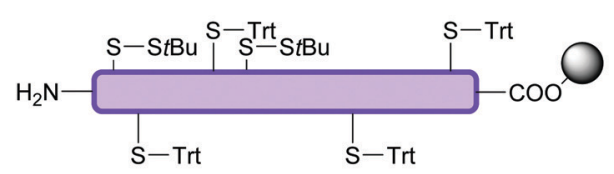

Linear protected linaclotide i) $20 \% \mathrm{BME}, 0.1 \mathrm{M} \mathrm{NMM}$ in DMF

StBu deprotection

ii) TFA:TIS:DCM (1:5:94)

Resin cleavage

iii) $5 \% \mathrm{DMSO}, \mathrm{pH} 8$

Disulfide formation

iv) TFA:TIS: $\mathrm{H}_{2} \mathrm{O}(95: 2.5: 2.5)$

Trt deprotection

v) $5 \% \mathrm{DMSO}, \mathrm{pH} 8$

Disulfide formation

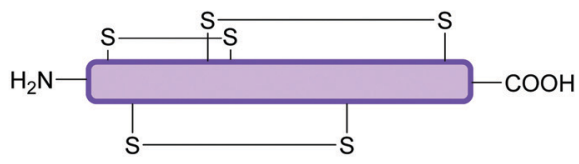

Linaclotide $=-\underline{C C E Y} \underline{C C N P A} \underline{C} T G \underline{C} Y-$

Fig. 39 (a) Cys thiol protection with the tert-butylsulphenyl (StBu) protecting group. (b) Reduction of Cys(StBu) and perfluoroaryl linker-containing peptide with TCEP in the presence of glutathione S-transferase (GST). Deprotection of Cys(StBu) yields the free thiol, which can then undergo macrocyclisation with the perfluoroaryl linker facilitated by GST. (c) Partial synthesis of $\mu$-conotoxin SIIIA using Cys(StBu). (d) Partial synthesis of $\alpha$-LvIA using Cys(StBu). (e) Synthesis of Linaclotide using Cys(StBu).

strongly acidic conditions (1 M TMFSA in TFA) and when $\mathrm{AgNO}_{3}$ is used; these conditions are used to deprotect $\mathrm{Cys}(\mathrm{Mob})$ and Cys(Acm) respectively, and thus (in a given case) deprotection of Cys(Pac) prior to these two groups has been recommended. ${ }^{6}$ As with Pocam, the group can be removed at the same time as an $N$ azido group with $\mathrm{Zn} / \mathrm{AcOH}$ for use in the thioester method of protein ligation. ${ }^{161}$ Peptides including the antimicrobial peptide tachyplesin could be synthesised using Cys(Pac) in standard Fmoc SPPS (Fig. 40c). ${ }^{161}$

The Pac protecting group has been employed in the semisynthesis of histone $\mathrm{H} 3$ bearing an $\mathrm{N}^{\varepsilon}$ trimethylated Lys residue ([Lys $\left.\left(\mathrm{Me}_{3}\right)^{9}\right] \mathrm{H} 3.1$, Fig. 40d). ${ }^{278}$ First, the C-terminal fragment of the peptide containing an $\mathrm{N}$-terminal Cys and two internal Cys residues (H3.1(26-135)) was recombinantly produced by gene expression and subsequent peptide production in E. coli. The $\mathrm{N}$-terminal Cys residue was then orthogonally protected with Thz, whereas both the internal Cys were protected with Pac. Conditions for deprotection of N-terminal Cys(Thz) using methoxyamine also lead to oxime ether formation at the ketone position of Pac, converting Pac to "PacN"; this, however, does not impact on downstream deprotection as PacN also shows lability to $\mathrm{Zn} / \mathrm{AcOH}$ similar to Pac. Deprotection of the N-terminal Cys(Thz), followed by CPE ligation with a suitable H3 N-terminal fragment and subsequent desulfurisation gave the dual Cys(PacN) protected peptide. Critically, the $\mathrm{Cys}(\mathrm{PacN})$ groups remained resistant to desulfurisation. Deprotection with $\mathrm{Zn}$ powder and 
a) N-Methyl-phenacyloxycarbamidomethyl<smiles>CC(C)NC(=O)[C@H](CSCN(C)C(=O)OCC(=O)c1ccccc1)NC(C)C</smiles>

b) Phenacyl

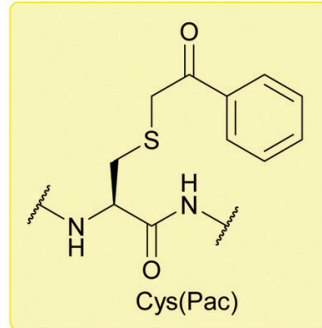

c) Synthesis of tachyplesin
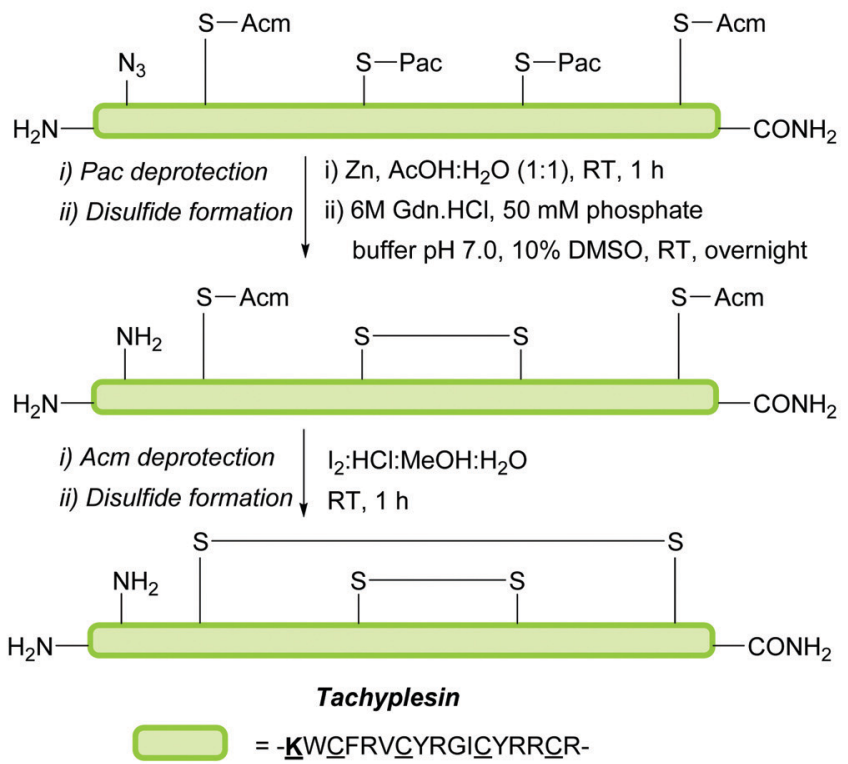

d) Synthesis of histone H3.1

H3.1 (26-135)
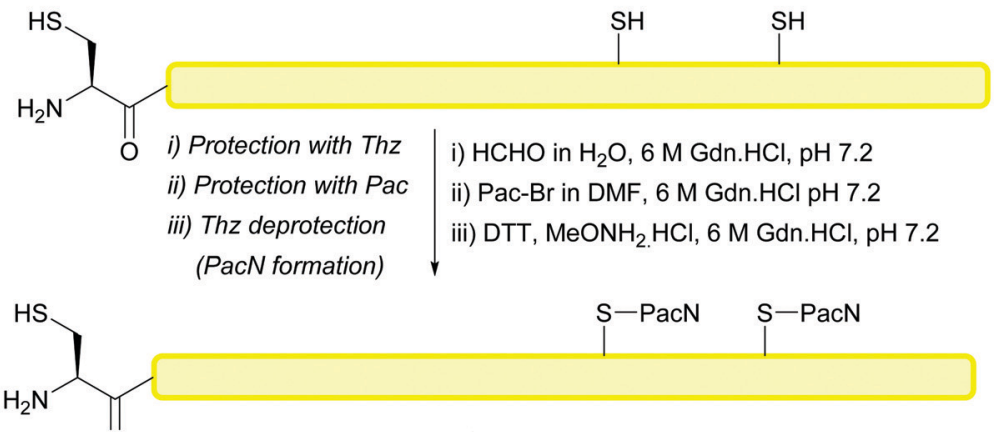

iv) CPE ligation

iv)

v) Desulfurisation

MPAA, TCEP, 6 M Gdn. $\mathrm{HCl}$, pH 8.0
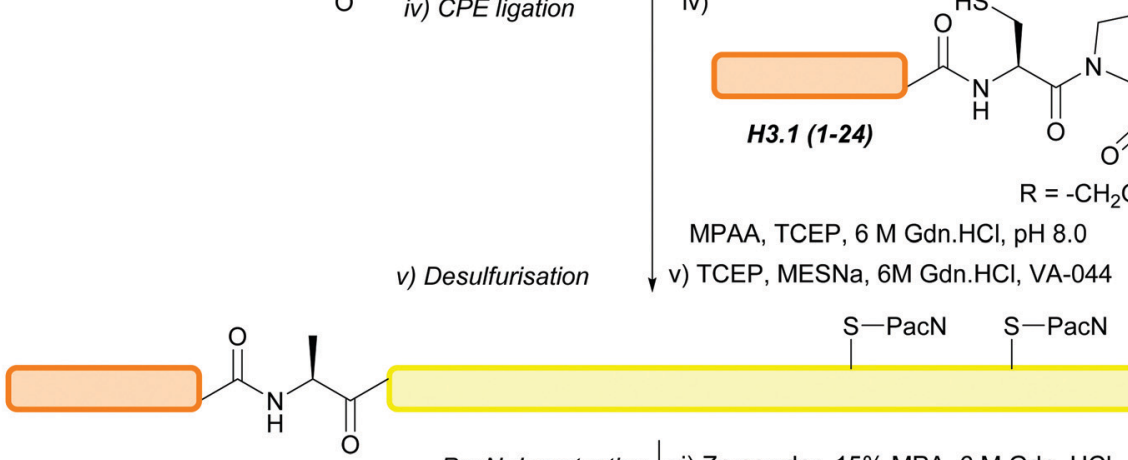

H3.1 (Ala35)

PacN deprotection vi) Zn powder, 15\% MPA, 6 M Gdn. $\mathrm{HCl}$

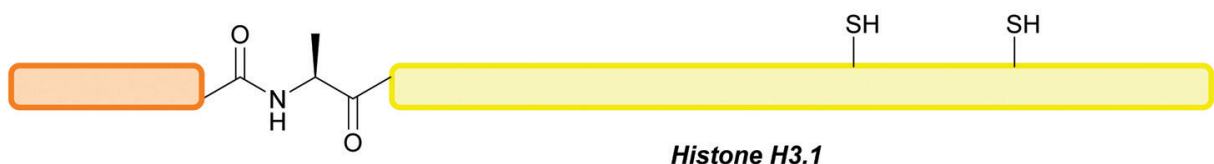

Histone $H 3.1$

$=-$ RTKQTARKSTGGKAPRKQLATKAARKSAPATGGVKKPHRYRPGTVALREIRRYQKSTELLIRKLPF QRLVREIAQDFKTDLRFQSSAVMALQEACEAYYVGLFEDTNLCAAIHAKRVTIMPKDIQLARRIRGERA-

Fig. 40 (a) Cys thiol protection with the $N$-methyl-phenacyloxycarbamidomethyl (Pocam) protecting group. (b) Cys thiol protection with the phenacyl (Pac) protecting group. (c) Synthesis of tachyplesin using Cys(Pac). (d) Semi-synthesis of histone H3.1 using Cys(Pac). 
15\% 3-mercaptopropionic acid (MPA) in $\mathrm{H}_{2} \mathrm{O}$ with $6 \mathrm{M}$ Gdn yielded the product $\left[\mathrm{Lys}\left(\mathrm{Me}_{3}\right)^{9}\right] \mathrm{H} 3.1$ histone. ${ }^{278}$ Similarly, it has been shown that $\mathrm{Cys}(\mathrm{Pac})$ can be used in conjunction with recombinantly generated protein segments for the traceless semisynthesis of human small heat shock protein (Hsp27) and a lipidated variant of murine prion protein (Prp). ${ }^{162}$ Pac proved compatible both with installation into thioester-containing peptides, and in radical desulfurisation steps. Cys(Trt) was also investigated; however, in this case, desulfurisation of Cys(Trt) containing Hsp27 proved lead to a mixture of products. ${ }^{162}$

5.10.11 S-Iso-propyl (SiPr). The $S$-iso-propyl (SiPr) Cys protecting group (Fig. 41a) has been used in the Boc solution phase synthesis of disulfide containing peptides. ${ }^{279}$ More recently, the group has been featured in one of the first examples of using orthogonal Cys protecting groups for construction of multi-drug containing ADCs. ${ }^{114}$ In this example, a dual protected Cys-based carrier containing $\mathrm{Cys}(\mathrm{SiPr})$ and $\mathrm{Cys}(\mathrm{Acm})$ was constructed using Fmoc SPPS, and furnished with a "self stabilising" maleimide linker. The carrier could then be used to site specifically modify free Cys (generated from reduction of interchain disulfides) of an immunoglobulin G (IgG), specifically CD30-directed antibody cAC10 (Fig. 41b). Orthogonal deprotection of Cys(SiPr) with TCEP accompanied by sequential conjugation of maleimide-linked monomethyl auristatin F (MMAF) give rise to the installation of one drug molecule per carrier. This was then followed by Cys(Acm) deprotection with $\mathrm{HgO}(\mathrm{Ac})_{2}$ and addition of monomethyl auristatin E (MMAE), allowing for the installation of two different drug moieties per conjugated carrier, resulting in an average of 16 drugs per antibody ( $8 \mathrm{MMAF}+8 \mathrm{MMAE}$, Fig. 41c). The in vitro and in vivo activities of the dual-auristatin ADC were then compared to cAC10 loaded with only MMAF or cAC10 loaded with only MMAE. The dual-auristatin ADC displayed both resistance to drug exportation via multi drug resistance (MDR) exporters due to MMAF, and cell permeability due to MMAE, combining the advantageous properties of both drugs onto a single ADC. ${ }^{114}$

5.10.12 Dimethoxyphenylthio ( $S$-Dmp) and 2,4,6-trimethoxyphenylthio ( $S$-Tmp). The dimethoxyphenylthio ( $S$-Dmp, Fig. 42a) and 2,4,6-trimethoxyphenylthio ( $S$-Tmp, Fig. 42b) groups were synthesised in 2012 as highly-labile replacements for the $\mathrm{StBu}$ group in Fmoc SPPS. ${ }^{163}$ Both groups can be removed using NMM $(0.1 \mathrm{M})$ with either $20 \% \mathrm{BME}$ in DMF or $5 \%$ DTT in DMF in $5 \mathrm{~min}$. In contrast, removal of the $\mathrm{StBu}$ protecting group using the aforementioned conditions required $3 \mathrm{~h}$ of incubation with $\mathrm{BME}$, whereas little to no deprotection of $\mathrm{StBu}$ was observed when using DTT. Both the $S$-Dmp and $S$-Tmp groups were noted to be compatible to Fmoc removal conditions (20\% piperidine in DMF, 4 h). Model tripeptides containing either group displayed stability towards conditions for resin cleavage (95\% TFA, 1 h, RT); however, partial instability (8-13\% deprotection) was observed following treatment with TFA/TIS/ $\mathrm{H}_{2} \mathrm{O}(95: 2.5: 2.5)$ at RT when

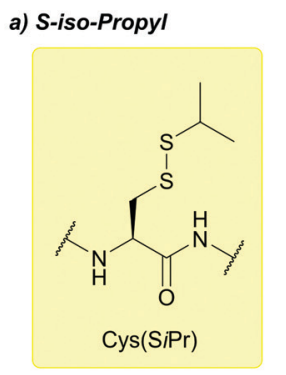

b) cAC10 antibody conjugated with carrier
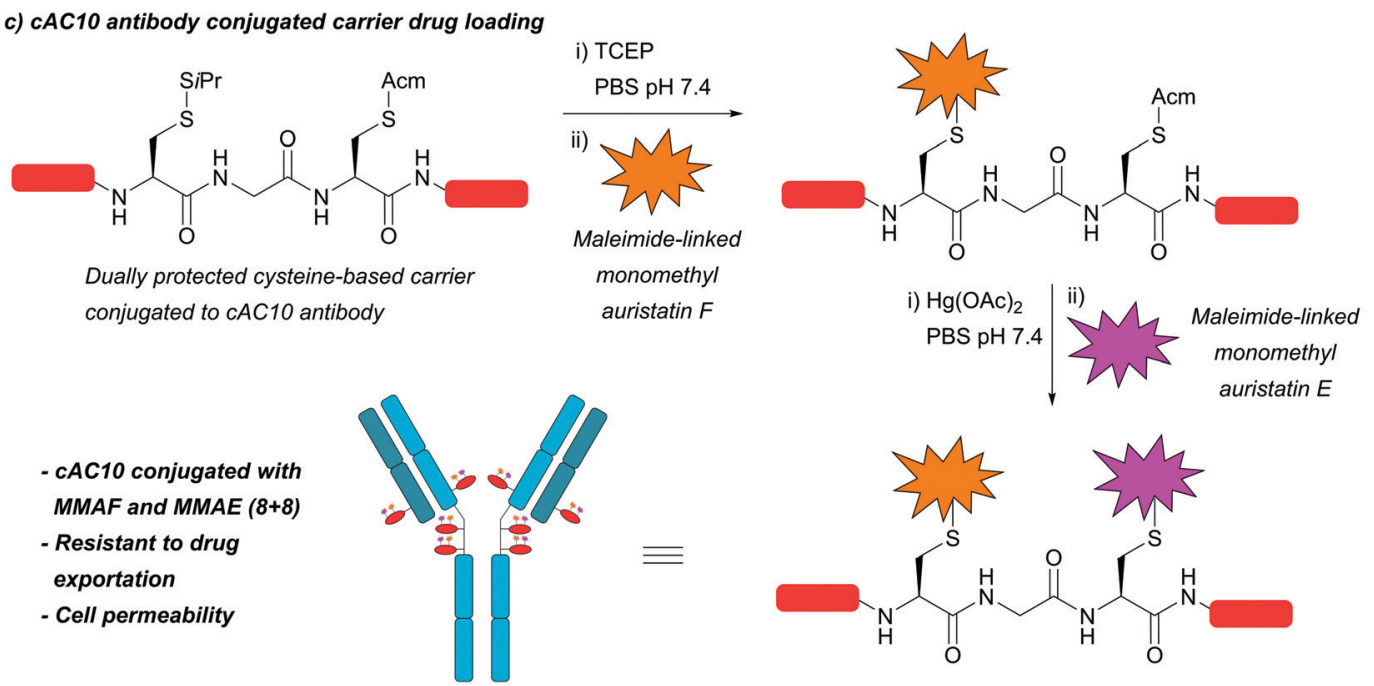

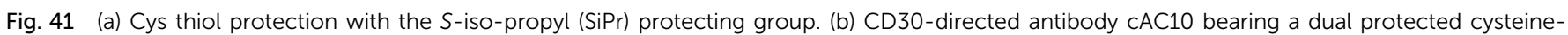
based carrier containing Cys(SiPr) and Cys(Acm). (c) Construction of CAC10 conjugated with MMAF and MMAE (8 + 8) using Cys(SiPr). 
applied to oxytocin synthesis. This could be avoided by using a more acid-labile resin, avoiding the requirement for high TFA concentrations. As with $\mathrm{StBu}, S$-Dmp and $S$-Tmp should be deprotected first in orthogonal protecting group strategies (which usually results in deprotection prior to TFA cleavage from the resin). The use of $S$-Dmp and $S$-Tmp has been demonstrated practically, with sample syntheses of oxytocin on-resin, and T22 carried out. ${ }^{163}$ Both groups produced better yields than the corresponding peptides synthesised using StBu. Out of the two groups, $S$-Tmp showed higher stability and produced purer peptides, and is therefore recommended for use. ${ }^{163}$ In addition to using DTT, deprotection of the S-Dmp group has also been demonstrated using a novel reducing agent 2-(dibenzylamino)butane-1,4-dithiol (DABDT). ${ }^{164}$ The $S$-Tmp protecting group has since been used in conjunction with $N$-chlorosuccinimide in the on-resin, regioselective synthesis of $\alpha$-conotoxin SI (Fig. 42c), ${ }^{280}$ and very recently in the synthesis of a disulfide containing, truncated neuropeptide Y (NPY) analogue. ${ }^{281}$

5.10.13 Sec-isoamyl mercaptan (SIT) and 2-methyloxolane3-thiol (MOT). 3-Methyl-2-butanethiol/sec-isoamyl mercaptan (SIT, Fig. 42d) and 2-methyloxolane-3-thiol (MOT, Fig. 42e) have very recently been described as disulfide-based protecting groups for Cys. ${ }^{165}$ In contrast to other disulfide-based protecting groups, SIT and MOT show greater thiol lability compared to StBu (and are therefore easier to remove), but display greater stability towards $20 \%$ piperidine than $S$-Dmp. Deprotection of both SIT and MOT could be achieved with BME in DMF (1:4) and 0.1 M DIEA. Alternatively, 20 equiv. DTT in MeCN with 5\% DIEA could also be used to remove both groups; the rate of deprotection was further enhanced by addition of $5 \% \mathrm{H}_{2} \mathrm{O}$, with SIT and MOT fully removed within 40 and 20 min respectively. Treatments of 5 equiv. DTT in DMF : DIEA : $\mathrm{H}_{2} \mathrm{O}$ (95:2.5:2.5, three treatments, $10 \mathrm{~min}$ ) was also sufficient for deprotection of Cys(SIT) and Cys(MOT) located within Asn(Trt)-Cys(SIT/MOT)Asn(Trt) tripeptides. Both of these groups have been successfully used in the synthesis of vasopressin via Fmoc SPPS, although minor thiol deprotection (ca. 2\%) of protected vasopressin during SPPS was noted for the more labile MOT group. Furthermore, the SIT protecting group could be used in microwave-assisted synthesis of vasopressin. ${ }^{165}$

5.10.14 2-Pyridinesulfenyl (S-Pyr). The 2-pyridinesulfenyl $(S$-Pyr) group is a thiol-labile protecting group (Fig. 43$){ }^{282}$ It is suitable for use in Boc SPPS ${ }^{283}$ and is stable to acidic conditions, e.g. 1 M TFMSA in TFA-anisole $\left(10: 1,2 \mathrm{~h}, 0{ }^{\circ} \mathrm{C}\right) .{ }^{4} S$-Pyr can also act as an activating group; free thiols can attack the activated sulfur atom, displacing $S$-Pyr to generate disulfide containing peptides. ${ }^{142,284}$ This has recently been shown in the synthesis of sialic acidcontaining insulin analogues, referred to as "Sialic-Ins". 285

\subsection{Safety-catch protecting groups}

5.11.1 4,4-Bis(dimethylsulfinyl)benzhydryl (Msbh). The 4,4-bis(dimethylsulfinyl)benzhydryl (Msbh) protecting group is an example of a 'safety-catch' protecting group - that is, it is stable to a particular set of conditions until the group undergoes a specific reaction. In the case of Msbh, it is stable to acidic (TFA, HF), oxidative and reductive conditions until its electron-withdrawing sulfoxide groups are reduced. It is both reduced and removed by a cocktail of $\mathrm{NH}_{4} \mathrm{I} / \mathrm{DMS} / \mathrm{TFA}$. Iodine/iodosulfonium ions are produced as a a) S-Dimethoxyphenylthio

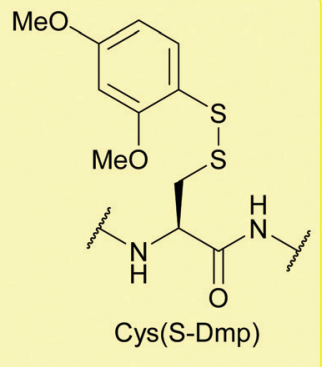

b) S-Trimethoxyphenylthio

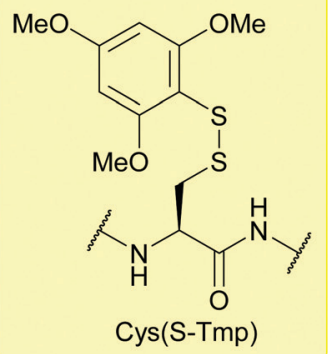

c) Partial synthesis of $\alpha$-Conotoxin SI
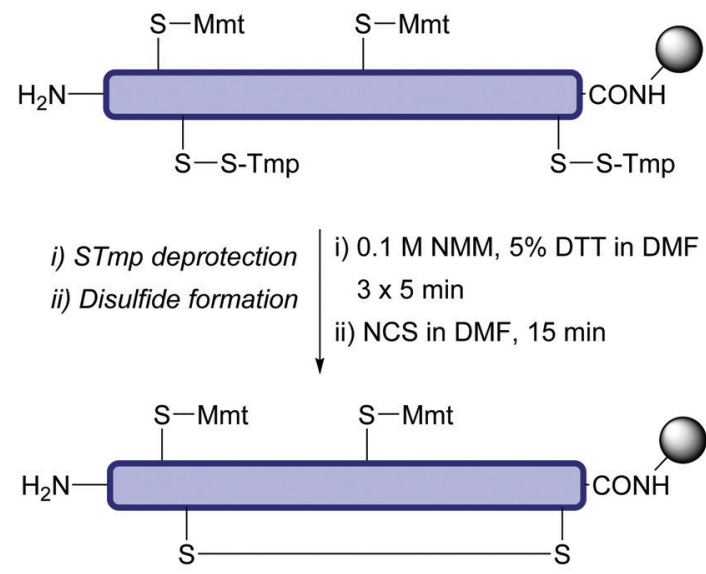

Resin bound protected disulfide containing $\alpha$-Conotoxin SI

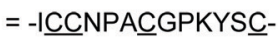

d) Sec-isoamyl mercaptan

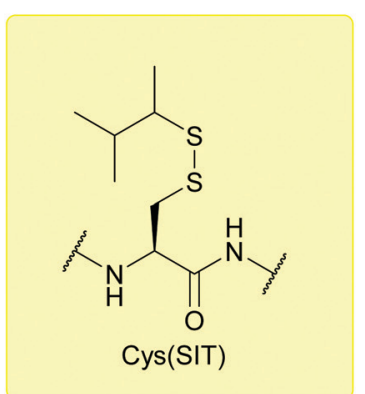

e) 2-Methyloxolane-3-thiol

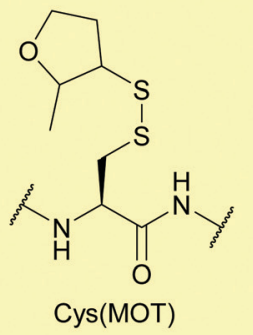

Fig. 42 (a) Cys thiol protection with the dimethoxyphenylthio (S-Dmp) protecting group. (b) Cys thiol protection with the 2,4,6-trimethoxyphenylthio (S-Tmp) protecting group. (c) Partial synthesis of synthesis of $\alpha$-conotoxin SI using Cys(S-Tmp). (d) Cys thiol protection with the sec-isoamyl mercaptan (SIT) protecting group. (e) Cys thiol protection with the 2-methyloxolane-3-thiol (MOT) protecting group. 
2-Pyridinesulfenyl

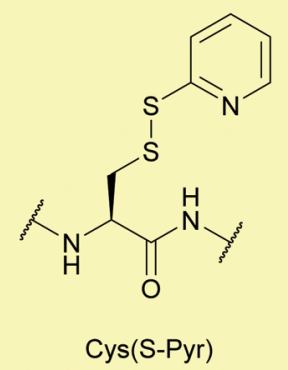

Fig. 43 Cys thiol protection with the 2-pyridinesulfenyl (S-Pyr) protecting group. side product of the reaction and thus oxidise the liberated thiols directly to the disulfide (Fig. 44a). Msbh is fully compatible with a range of other protecting groups, such as Trt, Acm, Meb and Mob. ${ }^{166}$

$\mathrm{NH}_{4} \mathrm{I} / \mathrm{TFA}$ treatment is incompatible with Trp-containing peptides, as Trp undergoes a number of side reactions (due to the presence of $\mathrm{I}_{2}$ ) unless the indole nitrogen is protected with a formyl group. This is standard in Boc SPPS and thus should not present a significant issue in this case. The formyl group is, however, removed by piperidine treatment so if Fmoc SPPS is being used alternative sulfoxide reduction methods or Trp protecting groups are needed. ${ }^{166}$ Msbh has been used in the regioselective synthesis of human hepcidin (Fig. 44b), providing an orthogonal strategy for the synthesise of peptides containing four disulfide bonds. ${ }^{166}$ It has also been theorised to

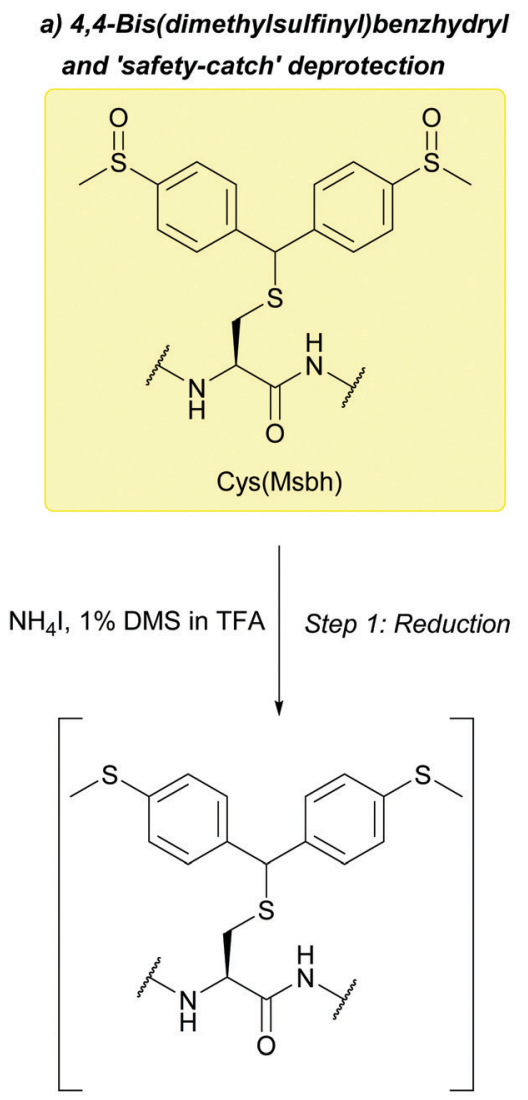

Step 2: Acidolytic cleavage

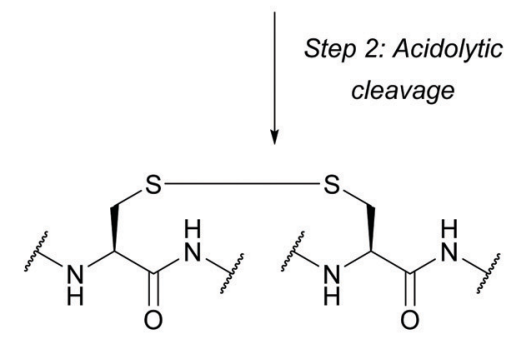

Disulfide containing peptide b) Regioselective disulfide formation of human hepcidin

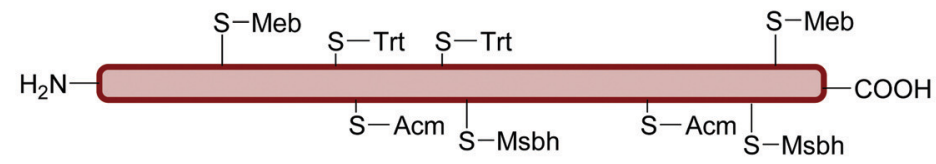

\begin{tabular}{l|l} 
Trt deprotection & i) TFA:TIPS: $\mathrm{H}_{2} \mathrm{O}(95: 2.5: 2.5)$ \\
Disulfide formation & ii) DMSO:AcOH: $\mathrm{H}_{2} \mathrm{O}(30: 5: 65)$
\end{tabular}

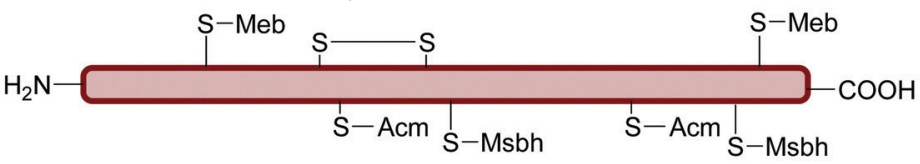

\begin{tabular}{l|l} 
Acm deprotection & Disulfide formation
\end{tabular} \mid $\mathrm{I}_{2} \mathrm{H}_{2} \mathrm{O}: \mathrm{MeCN}(50: 50), 0.1 \%$ TFA

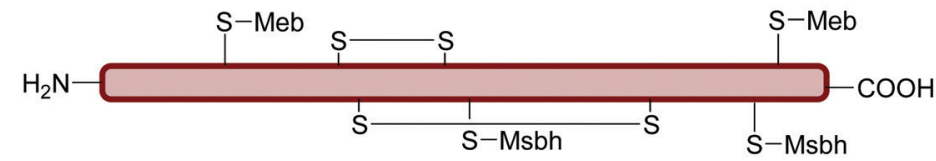

Meb deprotection $\quad$ i) HF:p-cresol (9:1)

Disulfide formation $\downarrow$ ii) DMSO:AcOH: $\mathrm{H}_{2} \mathrm{O}(30: 5: 65)$

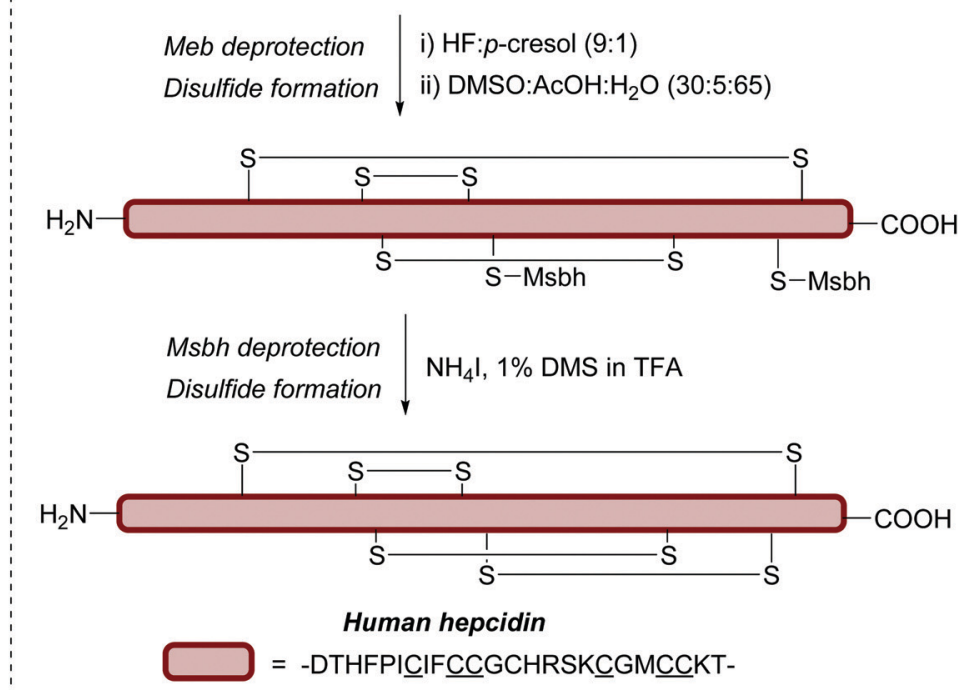

Fig. 44 (a) Cys thiol protection with the 4,4-bis(dimethylsulfinyl)benzhydryl (Msbh) protecting group. Treatment with a cocktail of NH ${ }_{4} / / \mathrm{DMS} \mathrm{TFA}$ results in initial reduction of the Msbh protecting group, followed by acid-mediated cleavage and subsequent iodine-mediated oxidation to yield disulfide containing peptides. (b) Partial synthesis and regioselective disulfide formation of human hepcidin using Cys(Msbh). 
be key to the regiospecific construction of peptides containing five or more disulfide bonds, a feat that is yet to be accomplished. ${ }^{5}$

\section{Conclusions}

Methods for the protection, and subsequent deprotection, of the thiol side chain of Cys has enabled a vast array of peptide and protein chemistry over the last 70 years. The development of protecting groups that are orthogonal to one another in particular has proved critical to facilitating the synthesis of complex products such as disulfide rich peptides. Although there are now numerous protecting strategies that have been reported for Cys, it is likely that research and development on the subject will continue over the course of the coming years. This will undoubtedly involve taking well-established Cys protecting groups, and developing new deprotection conditions that offer advantages over established methods. For example, protecting groups originally reported decades ago for use in Boc peptide synthesis such as Dpm, Thp, and SiPr, have greatly benefitted from being revisited for use in Fmoc peptide synthesis, both from the perspective of developing new deprotection protocols, and the application of such groups. Transition metal chemistry applied to Cys protecting group chemistry in recent years has also yielded new, milder conditions to deprotect protecting groups such as Trt, $t \mathrm{Bu}$, Acm, and Thz, or improved deprotection protocols in the case of Allocam. Alternatively, the development of new protecting groups with their own set of deprotection conditions will also likely prove paramount to furthering Cys-based peptide and protein chemistry. Protecting groups such as Mbom and Ddm offer C-terminal Cys-containing peptides with reduced racemisation side-reactions. Redesigning previously reported protecting group scaffolds has also led to the development of new protecting groups that are significantly easier to deprotect, as seen with $S$-Dmp/S-Tmp and SIT/MOT. Furthermore, newer protecting groups with unique "turn on/off" deprotection conditions, such as $\mathrm{Hmb}^{\text {off/on }}$ and Msbh, will likely see further development in challenging peptide synthesis. For example, regioselective synthesis of peptides with greater than four disulfide bonds has not yet been reported; novel protecting groups will likely prove critical to achieving this.

Finally, there is an increasing desire to make the process of SPPS more "green", which currently uses toxic and environmentally unfriendly reagents such as piperidine, DMF, and DCM. ${ }^{286}$ This will require protecting group chemistry that is compatible with greener reagents, such as water/aqueous-based solvent systems. Enzymatically labile protecting groups such as Phacm, or photolabile groups such as NDBF, have already been successfully utilised in this context; future protecting groups with similar properties will likely prove important in the "greening" of SPPS. We additionally anticipate the field of peptide chemistry and growing field of protein bioconjugation will likely benefit each other in the coming years. In contrast to conventional peptide synthesis, protein bioconjugation/deconjugation is, by design, carried out in benign/ environmentally friendly aqueous systems in a site-specific manner. Additionally, there is a continuing search for new methodology

for use in bioconjugation; although these must yield stable conjugates, strategies whereby the bioconjugate can be released in a controlled manner offers huge potential in applications such as controlled drug release. This interplay has already been demonstrated with Cys protecting groups that have since been applied to bioconjugation, such as Acm, Scm, Thz, and SiPr. Similarly, Suc, which is routinely used in bioconjugation, has very recently been demonstrated as a Cys protecting group in peptide synthesis. It is likely other bioconjugation strategies will also be applied to peptide synthesis in the coming years.

We have reviewed, analysed, and discussed over 60 individual protecting groups for the thiol group of Cys. We hope that this review provides a useful resource for peptide and protein chemists research, and encourages further research into both old and new Cys protecting groups.

\section{Abbreviations}

\section{2-Moxan \\ 2,6-diMeOBn \\ $4 \mathrm{MeO}-2 \mathrm{MeBn}$ \\ 5-Npys \\ 7,8BCMCMOC}

Acm

$\mathrm{AcOH}$

Ad/1-Ada

ADC

Alloc

Allocam

AgOAc

AgOTf

Arg

Bam

BCMACMOC

Bhc

BME

Boc

Bom

BSA

$\mathrm{Bu}_{3} \mathrm{SnH}$

$\mathrm{Bzl} / \mathrm{Bn}$

C4MNB

CAN

CDMNB

CUAAC

CPE

CPI

Cys

Dbs/Sub

DBU

DCC

DCM
2-methoxy-9H-xanthen-9-yl

2,6-dimethoxylbenzyl

4-methoxy-2-methylbenzyl

5-nitro-2-pyridinesulfenyl

[7,8-bis(carboxymethoxy)coumarin-4-yl]methoxycarbonyl

acetamidomethyl

acetic acid

1-adamantyl

antibody drug conjugate

allyloxycarbonyl

allyloxycarbonylaminomethyl

silver acetate

silver trifluoromethanesulfonate

arginine

benzamidomethyl

[7-bis(carboxymethyl)-amino-coumarin-

4-yl]methoxycarbonyl

6-bromo-7-hydroxycoumarin

2-mercaptoethanol

tert-butyloxycarbonyl

benzyloxymethyl

bovine serum albumin

tributyltin hydride

benzyl

$\alpha$-carboxy-4-methoxy-2-nitrobenzyl

ceric ammonium nitrate

$\alpha$-carboxy-4-methoxy-2-nitrobenzyl

copper(I)-catalysed alkyne-azide cycloaddition

cysteinylprolyl ester

cysteinylprolyl imide

cysteine

5-dibenzosuberyl

1,8-diazabicyclo[5.4.0]undec-7-ene

dicyclohexylcarbodiimide

dichloromethane 


\begin{tabular}{|c|c|c|c|}
\hline Ddm/4,4'-diMeODpm & 4,4'-dimethoxydiphenylmethyl & Mob/MBzl & 4-methoxybenzyl \\
\hline DIEA & $N, N$-diisopropylethylamine & MOT & 2-methyloxolane-3-thiol \\
\hline $\mathrm{DMB}$ & 3,4-dimethylbenzyl & MPAA & 4-mercaptophenylacetic acid \\
\hline \multirow[t]{2}{*}{ Dmbm } & (4,6-dimethoxy-2,2-dimethyl-2,3- & MPS & mercaptopropiosulfonic acid \\
\hline & dihydrobenzofuran-7-yl)methanol & Mpt & dimethylphosphinothioyl \\
\hline DMF & dimethylformamide & Msbh & 4,4-bis(dimethylsulfinyl)benzhydryl \\
\hline DMS & dimethyl sulfide & Mtt & 4-methyltrityl \\
\hline DMSO & dimethylsulfoxide & NCL & native chemical ligation \\
\hline Dnpe & 2-(2,4-dinitrophenyl)ethyl & NDBF & nitrodibenzofuran \\
\hline DPDS & 2,2'-dipyridyl disulfide & NDMBA & 1,3-dimethylbarbituric acid \\
\hline Dpm & diphenylmethyl & Nin & ninhydrin \\
\hline DSF & disulfiram & NMM & $N$-methylmorpholine \\
\hline DTC & diethyldithiocarbamate & $\mathrm{NpsCl}$ & 2-nitrophenylsulfenyl chloride \\
\hline DTNB & 5,5'-dithiobis-(2-nitrobenzoic acid) & Npys & 3-nitro-2-pyridinesulfenyl \\
\hline DTNP & 2,2'-dithiobis(5-nitropyridine) & OMe-NDBF & methoxy-nitrodibenzofuran \\
\hline DTP & 2,2'-dithiodipyridine & $o \mathrm{NB}$ & 2-nitrobenzyl \\
\hline DTT & dithiothreitol & $o \mathrm{NV}$ & 2-nitroveratryl \\
\hline EDT & ethane-1,2-dithiol & $p \mathrm{AB}$ & para-aminobenzyl \\
\hline EDTA & ethylenediaminetetraacetic acid & Pac & phenacyl \\
\hline EETI-II & Ecballium elaterium trypsin inhibitor II & PAL & peptide-amide-linker \\
\hline EGFP & enhanced green fluorescent protein & $\mathrm{PB}$ & phosphate buffer \\
\hline $\mathrm{EtOH}$ & ethanol & $\mathrm{Pbfm} / \mathrm{Pmbf}$ & 2,2,4,6,7-pentamethyl-2,3- \\
\hline $\mathrm{Fm}$ & 9-fluorenylmethyl & & dihydrobenzofuran-5-methyl \\
\hline Fmoc & 9-fluorenylmethoxycarbonyl & pBNP & porcine brain natriuretic peptide \\
\hline \multirow[t]{2}{*}{ Fnam } & {$\left[N-\left[2,3,5,6\right.\right.$-tetrafluoro-4-( $N^{\prime}$-piperidino)- } & PBS & phosphate-buffered saline \\
\hline & phenyl], $N$-allyloxycarbonyl]-aminomethyl & {$[\mathrm{Pd}(\text { allyl }) \mathrm{Cl}]_{2}$} & allylpalladium(II) chloride dimer \\
\hline Fsam & $\begin{array}{l}S \text {-[N-[2,3,5,6-tetrafluoro-4-(phenylthio)- } \\
\text { phenyl], } N \text {-allyloxycarbonyl]-aminomethyl }\end{array}$ & $\mathrm{PdCl}_{2}\left(\mathrm{PPh}_{3}\right)_{2}$ & $\begin{array}{l}\text { bis(triphenylphosphine)palladium(II) } \\
\text { dichloride }\end{array}$ \\
\hline GBP & growth-blocking peptide & $\mathrm{Pd}(\mathrm{OAc})_{2}$ & palladium(II) acetate \\
\hline $\mathrm{Gdn} \cdot \mathrm{HCl}$ & guanidinium chloride & $\mathrm{Pd}\left(\mathrm{PPh}_{3}\right)_{4}$ & tetrakis(triphenylphosphine)palladium(0) \\
\hline GFP & green fluorescent protein & PFTase & protein farnesyltransferase \\
\hline GSH & glutathione & PGA & penicillin $\mathrm{G}$ acylase \\
\hline $\mathrm{H}_{2} \mathrm{O}$ & water & Phacm & phenylacetamidomethyl \\
\hline \multirow[t]{2}{*}{ HEPES } & (4-(2-hydroxyethyl)-1-piperazineethane- & $\mathrm{PhSiH}_{3}$ & phenylsilane \\
\hline & sulfonic acid) & Pmcm & 2,2,5,7,8-pentamethylchroman-6- \\
\hline \multirow[t]{2}{*}{ HEPPS } & 4-(2-hydroxyethyl)-1-piperazinepropane- & & methyl \\
\hline & sulfonic acid & $p$ NB & para-nitrobenzyl \\
\hline HFIP & hexafluoro-2-propanol & Pocam & $N$-methyl-phenacyloxycarbamidomethyl \\
\hline Hgm & hydroxyglycine-Acm & PTM & post-translational modification \\
\hline $\mathrm{Hg}(\mathrm{OAc})_{2}$ & mercury(II) acetate & Pym & 2-oxo-1-pyrrolidinyl)methyl \\
\hline His & histidine & RP-HPLC & reversed phase high-performance liquid \\
\hline $\mathrm{Hmb}$ & 2-hydroxy-4-methoxy benzyl & & chromatography \\
\hline hNP2 & defensin human neutrophil peptide-2 & $\mathrm{Sac}$ & $S$-allyl cysteine \\
\hline HOBt & 1-hydroxybenzotriazole & $\mathrm{Scm}$ & carbomethoxysulfenyl \\
\hline Hqm & hydroxyquinoline-Acm & $S$-Dmp & dimethoxyphenylthio \\
\hline Lys & lysine & SIT & sec-isoamyl mercaptan/3-methyl-2- \\
\hline MAA & 3-mercaptoacetic acid & & butanethiol \\
\hline mBhc & 6-bromo-7-hydroxy-3-methylcoumarin & $\mathrm{SiPr}$ & $S$-iso-propyl \\
\hline Mbom & 4-methoxybenzyloxymethyl & Snm & $\left(N^{\prime}\right.$-methyl- $N^{\prime}$-phenylcarbamoyl)sulfenyl \\
\hline Meb/4-MeBn/4-MeBzl & 4-methylbenzyl & $\mathrm{SO}_{2} \mathrm{R}$ & sulfonyl \\
\hline $\mathrm{MeCN}$ & acetonitrile & $\mathrm{SO}_{3} \mathrm{H}$ & sulfonic acid \\
\hline $\mathrm{MeOH}$ & methanol & SPPS & solid phase peptide synthesis \\
\hline $\mathrm{MeONH}_{2} \cdot \mathrm{HCl}$ & $O$-methylhydroxylamine & SprC & $S$-propargyl-cysteine \\
\hline MESNA & sodium 2-mercaptoethanesulfonate & $S$-Pyr & 2-pyridinesulfenyl \\
\hline Met & methionine & $\mathrm{St} \mathrm{Bu}$ & tert-butylsulphenyl \\
\hline Mmt & 4-methoxytrityl & $S$-Tmp & 2,4,6-trimethoxyphenylthio \\
\hline
\end{tabular}




$\begin{array}{ll}\text { Suc } & \text { succinimide } \\ \text { Tacm } & \text { trimethylacetamidomethyl } \\ \text { TBAF } & \text { tetrabutylammonium fluoride } \\ t \text { Bu } & \text { tert-butyl } \\ \text { TCEP } & \text { tris(2-carboxyethyl)phosphine } \\ \text { TES } & \text { triethylsilane } \\ \text { TFA } & \text { trifluoroacetic acid } \\ \text { TFE } & \text { tetrafluoroethylene } \\ \text { TFMSA } & \text { trifluoromethanesulfonic acid } \\ \text { THF } & \text { tetrahydrofuran } \\ \text { Thp } & \text { tetrahydropyranyl } \\ \text { Thz } & \text { thiazolidine } \\ \text { TIS } & \text { triisopropylsilane } \\ \text { Tmbm } & 4,5,6 \text {-trimethoxy-2,2-dimethyl-2,3- } \\ \text { Tmob } & \text { dihydrobenzofuran-7-methyl } \\ \text { TMSBr } & 2,4,6 \text {-trimethoxybenzyl } \\ \text { TMSOTf } & \text { bromotrimethylsilane } \\ & \text { trimethylsilyl } \\ \text { TMTr } & \text { trifluoromethanesulfonate } \\ \text { Tppts } & 4,4^{\prime}, 4^{\prime \prime} \text {-trimethoxytriphenylmethyl } \\ \text { Trp } & 3,3^{\prime}, 3^{\prime \prime} \text {-phosphanetriyltris(benzene- } \\ \text { Trt } & \text { sulfonic acid) trisodium salt } \\ \text { Trx } & \text { tryptophan } \\ \text { Tyr } & \text { trityl } \\ \text { VA-044 } & \text { thioredoxin } \\ & \text { tyrosine } \\ \text { UBL5 } & 2,2^{\prime} \text {-azobis[2-(2-imidazolin-2-yl)propane]- } \\ \text { Xan } & \text { dihydrochloride } \\ \text { upro } & \text { ubiquitin-like protein } 5 \\ & \text { pse-xanthen-9-yl } \\ & \end{array}$

\section{Author contributions}

R. J. S., C. M. and V. C. co-wrote the review and co-analysed the literature with R. J. S. and V. C. leading in writing and analysing.

\section{Conflicts of interest}

V. C. is a co-founder and director of the company ThioLogics.

\section{Acknowledgements}

We gratefully acknowledge the Leverhulme Trust (RPG-2017288, 176274) for funding R. J. S.

\section{References}

1 G. Sartori, R. Ballini, F. Bigi, G. Bosica, R. Maggi and P. Righi, Chem. Rev., 2004, 104, 199-250.

2 B. Ghosh and S. S. Kulkarni, Chem. - Asian J., 2020, 15, 450-462.

3 M. Schelhaas and H. Waldmann, Angew. Chem., Int. Ed. Engl., 1996, 35, 2056-2083.
4 A. Isidro-Llobet, M. Álvarez and F. Albericio, Chem. Rev., 2009, 109, 2455-2504.

5 R. He, J. Pan, J. P. Mayer and F. Liu, ChemBioChem, 2020, 21, 1101-1111.

6 T. M. Postma and F. Albericio, Eur. J. Org. Chem., 2014, 3519-3530.

7 B. J. Tombling, C. K. Wang and D. J. Craik, Angew. Chem., Int. Ed., 2020, 59, 11218-11232.

8 M. Góngora-Benítez, J. Tulla-Puche and F. Albericio, Chem. Rev., 2014, 114, 901-926.

9 D. Imhof, D. Roy and F. Albericio, Front. Chem., 2020, 8, 841.

10 V. du Vigneaud, C. Ressler, J. M. Swan, C. W. Roberts and P. G. Katsoyannis, J. Am. Chem. Soc., 1954, 76, 3115-3121.

11 V. du Vigneaud, C. Ressler, C. J. M. Swan, C. W. Roberts, P. G. Katsoyannis and S. Gordon, J. Am. Chem. Soc., 1953, 75, 4879-4880.

12 I. Vecchio, C. Tornali, N. L. Bragazzi and M. Martini, Front. Endocrinol., 2018, 9, 613.

13 J. G. McGivern, Neuropsychiatr. Dis. Treat., 2007, 3, 69-85.

14 M. Corsetti and J. Tack, United Eur. Gastroenterol. J., 2013, 1, 7-20.

15 U. Hennrich and M. Benešová, Pharmaceuticals, 2020, 13(3), 38.

16 A. Fra, E. D. Yoboue and R. Sitia, Front. Mol. Neurosci., 2017, 10, 167.

17 S. Verma, R. Dixit and K. C. Pandey, Front. Pharmacol., 2016, 7, 107.

18 C. D. Spicer and B. G. Davis, Nat. Commun., 2014, 5, 4740.

19 V. Chudasama, A. Maruani and S. Caddick, Nat. Chem., 2016, 8, 114-119.

20 N. Joubert, A. Beck, C. Dumontet and C. DenevaultSabourin, Pharmaceuticals, 2020, 13, 245.

21 P. Stathopoulos, S. Papas, C. Pappas, V. Mousis, N. Sayyad, V. Theodorou, A. G. Tzakos and V. Tsikaris, Amino Acids, 2013, 44, 1357-1363.

22 Y. Fujiwara, K. Akaji and Y. Kiso, Chem. Pharm. Bull., 1994, 42, 724-726.

23 D. Lelièvre, V. P. Terrier, A. F. Delmas and V. Aucagne, Org. Lett., 2016, 18, 920-923.

24 J. Lukszo, D. Patterson, F. Albericio and S. A. Kates, Lett. Pept. Sci., 1996, 3, 157-166.

25 S. Laps, G. Satish and A. Brik, Chem. Soc. Rev., 2021, 50, 2367-2387.

26 E. Fischer and E. Fourneau, Ber. Dtsch. Chem. Ges., 1901, 34, 2868-2877.

27 J. C. Sheehan and G. P. Hess, J. Am. Chem. Soc., 1955, 77, 1067-1068.

28 L. A. Carpino, J. Am. Chem. Soc., 1957, 79, 4427-4431.

29 F. C. McKay and N. F. Albertson, J. Am. Chem. Soc., 1957, 79, 4686-4690.

30 R. B. Merrifield, J. Am. Chem. Soc., 1963, 85, 2149-2154.

31 J. M. Palomo, RSC Adv., 2014, 4, 32658-32672.

32 L. A. Carpino and G. Y. Han, J. Am. Chem. Soc., 1970, 92, 5748-5749.

33 D. M. M. Jaradat, Amino Acids, 2018, 50, 39-68. 
34 M. Ahangarpour, I. Kavianinia, P. W. R. Harris and M. A. Brimble, Chem. Soc. Rev., 2021, 50, 898-944.

35 P. R. Hansen and A. Oddo, in Fmoc Solid-Phase Peptide Synthesis, ed. G. Houen, Springer New York, New York, NY, 2015, pp. 33-50.

36 V. Agouridas, O. El Mahdi, M. Cargoët and O. Melnyk, Bioorg. Med. Chem., 2017, 25, 4938-4945.

37 P. E. Dawson, T. W. Muir, I. Clark-Lewis and S. B. Kent, Science, 1994, 266, 776-779.

38 P. E. Dawson and S. B. H. Kent, Annu. Rev. Biochem., 2000, 69, 923-960.

39 D. Bang and S. B. H. Kent, Angew. Chem., Int. Ed., 2004, 43, 2534-2538.

40 T. C. Evans Jr., J. Benner and M.-Q. Xu, Protein Sci., 1998, 7, 2256-2264.

41 T. W. Muir, D. Sondhi and P. A. Cole, Proc. Natl. Acad. Sci. U. S. A., 1998, 95, LP6705-LP6710.

42 L. Z. Yan and P. E. Dawson, J. Am. Chem. Soc., 2001, 123, 526-533.

43 Q. Wan and S. J. Danishefsky, Angew. Chem., Int. Ed., 2007, 46, 9248-9252.

44 T. Kawakami and S. Aimoto, Chem. Lett., 2006, 76-77.

45 M. Yanase, K. Nakatsu, C. J. Cardos, Y. Konda, G. Hayashi and A. Okamoto, Chem. Sci., 2019, 10, 5967-5975.

46 G.-M. Fang, Y.-M. Li, F. Shen, Y.-C. Huang, J.-B. Li, Y. Lin, H.-K. Cui and L. Liu, Angew. Chem., Int. Ed., 2011, 50, 7645-7649.

47 G.-M. Fang, J.-X. Wang and L. Liu, Angew. Chem., Int. Ed., 2012, 51, 10347-10350.

48 J.-S. Zheng, S. Tang, Y.-K. Qi, Z.-P. Wang and L. Liu, Nat. Protoc., 2013, 8, 2483-2495.

49 Y. Qiao, G. Yu, K. C. Kratch, X. A. Wang, W. W. Wang, S. Z. Leeuwon, S. Xu, J. S. Morse and W. R. Liu, J. Am. Chem. Soc., 2020, 142, 7047-7054.

50 H. Hojo and S. Aimoto, Bull. Chem. Soc. Jpn., 1991, 64, 111-117.

51 H. Hojo, Y. Murasawa, H. Katayama, T. Ohira, Y. Nakahara and Y. Nakahara, Org. Biomol. Chem., 2008, 6, 1808-1813.

52 H. Katayama, H. Hojo, T. Ohira and Y. Nakahara, Tetrahedron Lett., 2008, 49, 5492-5494.

53 H. Hojo, Org. Biomol. Chem., 2016, 14, 6368-6374.

54 W. Hou, X. Zhang and C.-F. Liu, Trans. Tianjin Univ., 2017, 23, 401-419.

55 A. C. Conibear, E. E. Watson, R. J. Payne and C. F. W. Becker, Chem. Soc. Rev., 2018, 47, 9046-9068.

56 S. S. Kulkarni, J. Sayers, B. Premdjee and R. J. Payne, Nat. Rev. Chem., 2018, 2, 122.

57 V. Agouridas, O. El Mahdi, V. Diemer, M. Cargoët, J.-C. M. Monbaliu and O. Melnyk, Chem. Rev., 2019, 119, 7328-7443.

58 G. Barany and R. B. Merrifield, J. Am. Chem. Soc., 1977, 99, 7363-7365.

59 H. C. Hang, C. Yu, D. L. Kato and C. R. Bertozzi, Proc. Natl. Acad. Sci. U. S. A., 2003, 100, LP14846-LP14851.

60 S. S. Nguyen and J. A. Prescher, Nat. Rev. Chem., 2020, 4, 476-489.

61 E. M. Sletten and C. R. Bertozzi, Acc. Chem. Res., 2011, 44, 666-676.
62 P. Agarwal, B. J. Beahm, P. Shieh and C. R. Bertozzi, Angew. Chem., Int. Ed., 2015, 54, 11504-11510.

63 S. T. Laughlin, J. M. Baskin, S. L. Amacher and C. R. Bertozzi, Science, 2008, 320, 664-667.

64 R. Brabham and M. A. Fascione, ChemBioChem, 2017, 18, 1973-1983.

65 C. H. Kim, M. Kang, H. J. Kim, A. Chatterjee and P. G. Schultz, Angew. Chem., Int. Ed., 2012, 51, 7246-7249.

66 A. Deiters, D. Groff, Y. Ryu, J. Xie and P. G. Schultz, Angew. Chem., Int. Ed., 2006, 45, 2728-2731.

67 V. du Vigneaud, L. F. Audrieth and H. S. Loring, J. Am. Chem. Soc., 1930, 52, 4500-4504.

68 D. Andreu, F. Albericio, N. A. Solé, M. C. Munson and G. Barany, in Methods in Molecular Biology, Peptide Synthesis Protocols, ed. M. W. Pennington and B. M. Dunn, Human Press Inc., Totowa, NJ, 1994, vol. 35, pp. 91-169.

69 H. Yajima, N. Fujii, S. Funakoshi, T. Watanabe, E. Murayama and A. Otaka, Tetrahedron, 1988, 44, 805-819.

70 L. Zervas and I. Photaki, J. Am. Chem. Soc., 1962, 84, 3887-3897.

71 K. Barlos, D. Gatos, O. Hatzi, N. Koch and S. Koutsogianni, Int. J. Pept. Protein Res., 1996, 47, 148-153.

72 K. Akaji, K. Fujino, T. Tatsumi and Y. Kiso, J. Am. Chem. Soc., 1993, 115, 11384-11392.

73 D. Kobayashi, N. Naruse, M. Denda, A. Shigenaga and A. Otaka, Org. Biomol. Chem., 2020, 18, 8638-8645.

74 M. Mochizuki, S. Tsuda, K. Tanimura and Y. Nishiuchi, Org. Lett., 2015, 17, 2202-2205.

75 M. Góngora-Benítez, L. Mendive-Tapia, I. RamosTomillero, A. C. Breman, J. Tulla-Puche and F. Albericio, Org. Lett., 2012, 14, 5472-5475.

76 G. F. Holland and L. A. Cohen, J. Am. Chem. Soc., 1958, 80, 3765-3769.

77 I. Ramos-Tomillero, H. Rodriguez and F. Albericio, Org. Lett., 2015, 17, 1680-1683.

78 J. J. Pastuszak and A. Chimiak, J. Org. Chem., 1981, 46, 1868-1873.

79 S. Sakakibara, Y. Shimonishi, Y. Kishida, M. Okada and H. Sugihara, Bull. Chem. Soc. Jpn., 1967, 40, 2164-2167.

80 A. L. Schroll, R. J. Hondal and S. Flemer, J. Pept. Sci., 2012, 18, 1-9.

81 O. Nishimura, C. Kitada and M. Fujino, Chem. Pharm. Bull., 1978, 26, 1576-1585.

82 K. Akaji, T. Tatsumi, M. Yoshida, T. Kimura, Y. Fujiwara and Y. Kiso, J. Chem. Soc., Chem. Commun., 1991, 167-168.

83 N. Fujii, A. Otaka, S. Funakoshi, K. Bessho, T. Watanabe, K. Akaji and H. Yajima, Chem. Pharm. Bull., 1987, 35, 2339-2347.

84 F. Wu, J. P. Mayer, V. M. Gelfanov, F. Liu and R. D. Dimarchi, J. Org. Chem., 2017, 82, 3506-3512.

85 M. Jbara, S. Laps, M. Morgan, G. Kamnesky, G. Mann, C. Wolberger and A. Brik, Nat. Commun., 2018, 9, 3154.

86 E. J. Ste Marie and R. J. Hondal, J. Pept. Sci., 2018, 24, e3130.

87 N. Fujii, A. Otaka, T. Watanabe, A. Okamachi, H. Tamamura, H. Yajima, Y. Inagaki, M. Nomizu and K. Asano, J. Chem. Soc., Chem. Commun., 1989, 283.

88 A. Cuthbertson and B. Indrevoll, Org. Lett., 2003, 5, 2955-2957. 
89 S. Akabori, S. Sakakibara, Y. Shimonishi and Y. Nobuhara, Bull. Chem. Soc. Jpn., 1964, 37, 433-434.

90 K. M. Harris, S. Flemer and R. J. Hondal, J. Pept. Sci., 2007, 13, 81-93.

91 B. W. Erickson and R. B. Merrifield, J. Am. Chem. Soc., 1973, 95, 3750-3756.

92 D. Yamashiro, R. L. Noble and C. H. Li, J. Org. Chem., 1973, 38, 3561-3565.

93 N. Fujii, A. Otaka, S. Funakoshi, H. Yajima, O. Nishimura and M. Fujino, Chem. Pharm. Bull., 1986, 34, 869-872.

94 A. Otaka, H. Morimoto, N. Fujii, T. Koide, S. Funakoshi and H. Yajima, Chem. Pharm. Bull., 1989, 37, 526-528.

95 M. C. Munson, C. Garcia-Echeverria, F. Albericio and G. Barany, J. Org. Chem., 1992, 57, 3013-3018.

96 M. C. Munson, C. Garcia-Echeverria, F. Albericio and G. Barany, in Peptides Chemistry and Biology (Proceedings of the Twelfth American Peptide Symposium), ed. J. A. Smith and J. E. Rivier, Leiden, 1992, pp. 605-606.

97 T. Wöhr, F. Wahl, A. Nefzi, B. Rohwedder, T. Sato, X. Sun and M. Mutter, J. Am. Chem. Soc., 1996, 118, 9218-9227.

98 M. S. Y. Wong and K. A. Jolliffe, Aust. J. Chem. 2010, 63, 797-801. 99 T. M. Postma and F. Albericio, Org. Lett., 2014, 16, 1772-1775. 100 S. Tsuda, S. Masuda and T. Yoshiya, J. Org. Chem., 2020, 85, 1674-1679.

101 M. Pelay-Gimeno, A. Meli, J. Tulla-Puche and F. Albericio, J. Med. Chem., 2013, 56, 9780-9788.

102 Y. Han and G. Barany, J. Org. Chem., 1997, 62, 3841-3848. 103 O. Garcia, J. M. Bofill, E. Nicolas and F. Albericio, Eur. J. Org. Chem., 2010, 3631-3640.

104 H. Hibino and Y. Nishiuchi, Org. Lett., 2012, 14, 1926-1929. 105 I. Ramos-Tomillero, L. Mendive-Tapia, M. Góngora-Benítez, E. Nicolás, J. Tulla-Puche and F. Albericio, Molecules, 2013, 18, 5155-5162.

106 H. Hibino, Y. Miki and Y. Nishiuchi, J. Pept. Sci., 2014, 20, 30-35. 107 Y.-K. Qi, S. Tang, Y.-C. Huang, M. Pan, J.-S. Zheng and L. Liu, Org. Biomol. Chem., 2016, 14, 4194-4198.

108 D. F. Veber, J. D. Milkowski, R. G. Denkewalter and R. Hirschmann, Tetrahedron Lett., 1968, 9, 3057-3058.

109 S. Laps, H. Sun, G. Kamnesky and A. Brik, Angew. Chem., Int. Ed., 2019, 58, 5729-5733.

110 D. F. Veber, J. D. Milkowski, S. L. Varga, R. G. Denkewalter and R. Hirschmann, J. Am. Chem. Soc., 1972, 94, 5456-5461.

111 M. Muttenthaler, Y. G. Ramos, D. Feytens, A. D. de Araujo and P. F. Alewood, Biopolymers, 2010, 94, 423-432.

112 S. K. Maity, M. Jbara, S. Laps and A. Brik, Angew. Chem., Int. Ed., 2016, 55, 8108-8112.

113 N. Kamo, G. Hayashi and A. Okamoto, Org. Lett., 2019, 21, 8378-8382.

114 M. R. Levengood, X. Zhang, J. H. Hunter, K. K. Emmerton, J. B. Miyamoto, T. S. Lewis and P. D. Senter, Angew. Chem., Int. Ed., 2017, 56, 733-737.

115 J. Pless, Helv. Chim. Acta, 1976, 59, 499-512.

116 P. K. Chakravarty and R. K. Olsen, J. Org. Chem., 1978, 43, 1270-1271.

117 Y. Kiso, M. Yoshida, Y. Fujiwara, T. Koimura, M. Shimokura and K. Akaji, Chem. Pharm. Bull., 1990, 38, 673-675.
118 M. Ueki and K. Shinozaki, Bull. Chem. Soc. Jpn., 1983, 56, 1187-1191.

119 M. Ueki, T. Ikeo, K. Hokari, K. Nakamura, A. Saeki and H. Komatsu, Bull. Chem. Soc. Jpn., 1999, 72, 829-838.

120 Y. Kiso, M. Yoshida, T. Kimura, Y. Fujiwara and M. Shimokura, Tetrahedron Lett., 1989, 30, 1979-1982.

121 M. Yoshida, T. Tatsumi, Y. Fujiwara, S. Iinuma, T. Kimura, K. Akaji and Y. Kiso, Chem. Pharm. Bull., 1990, 38, 1551-1557.

122 M. Bodanszky and M. A. Bednarek, Int. J. Pept. Protein Res., 1982, 20, 434-437.

123 M. Ruiz-Gayo, F. Albericio, E. Pedroso and E. Giralt, J. Chem. Soc., Chem. Commun., 1986, 1501.

124 M. Royo, C. García-Echeverría, E. Giralt, R. Eritja and F. Albericio, Tetrahedron Lett., 1992, 33, 2391-2394.

125 C. W. West, M. A. Estiarte and D. H. Rich, Org. Lett., 2001, 3, 1205-1208.

126 M. Royo, J. Alsina, E. Giralt, U. Slomcyznska and F. Albericio, J. Chem. Soc., Perkin Trans. 1, 1995, 1095-1102.

127 M. Góngora-Benítez, A. Basso, T. Bruckdorfer, M. Royo, J. Tulla-Puche and F. Albericio, Chem. - Eur. J., 2012, 18, 16166-16176.

128 F. Shen, Z. P. Zhang, J. Bin Li, Y. Lin and L. Liu, Org. Lett., 2011, 13, 568-571.

129 A. Loffet and H. X. Zhang, Int. J. Pept. Protein Res., 1993, 42, 346-351.

130 A. M. Kimbonguila, A. Merzouk, F. Guibé and A. Loffet, Tetrahedron Lett., 1994, 35, 9035-9038.

131 T. D. Kondasinghe, H. Y. Saraha, S. B. Odeesho and J. L. Stockdill, Org. Biomol. Chem., 2017, 15, 2914-2918.

132 P. Gomez-Martinez, A. Malanda Kimbonguila and F. Guibé, Tetrahedron, 1999, 55, 6945-6960.

133 P. Gomez-Martinez, F. Guibé and F. Albericio, Lett. Pept. Sci., 2000, 7, 187-194.

134 M. P. Exner, T. Kuenzl, T. M. T. To, Z. Ouyang, S. Schwagerus, M. G. Hoesl, C. P. R. Hackenberger, M. C. Lensen, S. Panke and N. Budisa, ChemBioChem, 2017, 18, 85-90.

135 J. Liu, R. Cheng, H. Wu, S. Li, P. G. Wang, W. F. DeGrado, S. Rozovsky and L. Wang, Angew. Chem., Int. Ed., 2018, 57, 12702-12706.

136 G. B. Vamisetti, G. Satish, P. Sulkshane, G. Mann, M. H. Glickman and A. Brik, J. Am. Chem. Soc., 2020, 142, 19558-19569.

137 S. Rather and H. T. Clarke, J. Am. Chem. Soc., 1937, 59, 200-206. 138 M. Jbara, S. K. Maity, M. Seenaiah and A. Brik, J. Am. Chem. Soc., 2016, 138, 5069-5075.

139 N. Naruse, D. Kobayashi, K. Ohkawachi, A. Shigenaga and A. Otaka, J. Org. Chem., 2020, 85, 1425-1433.

140 H. Katayama and S. Morisue, Tetrahedron, 2017, 73, 3541-3547.

141 C. T. Pool, J. G. Boyd and J. P. Tam, J. Pept. Res., 2004, 63, 223-234.

142 J. A. Karas, D. B. Scanlon, B. E. Forbes, I. Vetter, R. J. Lewis, J. Gardiner, F. Separovic, J. D. Wade and M. A. Hossain, Chem. - Eur. J., 2014, 20, 9549-9552.

143 A. B. Smith, S. N. Savinov, U. V. Manjappara and I. M. Chaiken, Org. Lett., 2002, 4, 4041-4044. 
144 N. Kotzur, B. Briand, M. Beyermann and V. Hagen, J. Am. Chem. Soc., 2009, 131, 16927-16931.

145 M. M. Mahmoodi, D. Abate-Pella, T. J. Pundsack, C. C. Palsuledesai, P. C. Goff, D. A. Blank and M. D. Distefano, J. Am. Chem. Soc., 2016, 138, 5848-5859.

146 M. M. Mahmoodi, S. A. Fisher, R. Y. Tam, P. C. Goff, R. B. Anderson, J. E. Wissinger, D. A. Blank, M. S. Shoichet and M. D. Distefano, Org. Biomol. Chem., 2016, 14, 8289-8300.

147 T. K. Bader, F. Xu, M. H. Hodny, D. A. Blank and M. D. Distefano, J. Org. Chem., 2020, 85, 1614-1625.

148 S. B. Gunnoo, A. Iyer, W. Vannecke, K. W. Decoene, T. Hebbrecht, J. Gettemans, M. Laga, S. Loverix, I. Lasters and A. Madder, Chem. Commun., 2018, 54, 11929-11932.

149 A. L. Schroll and G. Barany, J. Org. Chem., 1989, 54, 244-247.

150 W. Chan and P. White, Fmoc Solid Phase Peptide Synthesis: A Practical Approach, Oxford University Press, Oxford, 2000, p. 87.

151 A. Gosden, D. Stevenson and G. T. Young, J. Chem. Soc., Chem. Commun., 1972, 1123.

152 R. B. Merrifield, The Chemistry of Polypeptides, Springer US, Boston, MA, 1973.

153 I. Maugras, J. Gosteli, E. Rapp and R. Nyfeler, Int. J. Pept. Protein Res., 1995, 45, 152-156.

154 C. Rentier, K. Fukumoto, A. Taguchi and Y. Hayashi, J. Pept. Sci., 2017, 23, 496-504.

155 R. Matsueda, T. Kimura, E. T. Kaiser and G. R. Matsueda, Chem. Lett., 1981, 737-740.

156 R. J. Ridge, G. R. Matsueda, E. Haber and R. Matsueda, Int. J. Pept. Protein Res., 1982, 19, 490-498.

157 F. Albericio, D. Andreu, E. Giralt, C. Navalpotro, E. Pedroso, B. Ponsati and M. Rue-Gayo, Int. J. Pept. Protein Res., 1989, 34, 124-128.

158 F. Rabanal, W. F. DeGrado and P. L. Dutton, Tetrahedron Lett., 1996, 37, 1347-1350.

159 R. Eritja, J. P. Ziehler-Martin, P. A. Walker, T. D. Lee, K. Legesse, F. Albericio and B. E. Kaplan, Tetrahedron, 1987, 43, 2675-2680.

160 H. Katayama, Y. Nakahara and H. Hojo, Org. Biomol. Chem., 2011, 9, 4653.

161 H. Katayama and H. Hojo, Org. Biomol. Chem., 2013, 11, 4405-4413.

162 M. Matveenko, S. Hackl and C. F. W. Becker, ChemistryOpen, 2018, 7, 106-110.

163 T. M. Postma, M. Giraud and F. Albericio, Org. Lett., 2012, 14, 5468-5471.

164 S. N. Mthembu, A. Sharma, F. Albericio and B. G. de la Torre, Org. Lett., 2019, 21, 10111-10114.

165 A. Chakraborty, A. Sharma, F. Albericio and B. G. de la Torre, Org. Lett., 2020, 22, 9644-9647.

166 Z. Dekan, M. Mobli, M. W. Pennington, E. Fung, E. Nemeth and P. F. Alewood, Angew. Chem., Int. Ed., 2014, 53, 2931-2934.

167 R. Deslauriers, R. Walter and I. C. P. Smith, Biochem. Biophys. Res. Commun., 1972, 48, 854-859.

168 G. Flouret, W. Brieher, K. Mahan and L. Wilson, J. Med. Chem., 1991, 34, 642-646.

169 B. Banerji, M. Chatterjee, C. Prodhan and K. Chaudhuri, RSC Adv., 2016, 6, 112667-112676.
170 M. Amblard, J.-A. Fehrentz, J. Martinez and G. Subra, Mol. Biotechnol., 2006, 33, 239-254.

171 B. Kamber, A. Hartmann, K. Eisler, B. Riniker, H. Rink, P. Sieber and W. Rittel, Helv. Chim. Acta, 1980, 63, 899-915.

172 D. A. Pearson, M. Blanchette, M. Lou Baker and C. A. Guindon, Tetrahedron Lett., 1989, 30, 2739-2742.

173 E. E. Büllesbach and C. Schwabe, J. Biol. Chem., 1991, 266, 10754-10761.

174 J. Ying, X. Gu, M. Cai, M. Dedek, J. Vagner, D. B. Trivedi and V. J. Hruby, J. Med. Chem., 2006, 49, 6888-6896.

175 C. Fagundez, D. Sellanes and G. Serra, ACS Comb. Sci., 2018, 20, 212-219.

176 G. P. Schwartz and P. G. Katsoyannis, J. Chem. Soc., Perkin Trans, 1973, 1, 2894.

177 R. G. Hiskey and J. B. Adams, J. Org. Chem., 1966, 31, 2178-2183.

178 S. A. Khan and K. M. Sivanandaiah, Int. J. Pept. Protein Res., 1978, 12, 164-169.

179 A. Sharma, I. Ramos-Tomillero, A. El-Faham, E. Nicolas, H. Rodriguez, B. G. de la Torre and F. Albericio, ChemistryOpen, 2017, 6, 168-177.

180 A. Chimiak, in Proceedings of 5th European Peptide Symposium, 1962, p. 37.

181 X. Zhang, Y. Yang, M. Zhao, L. Liu, M. Zheng, Y. Wang, J. Wu and S. Peng, Eur. J. Med. Chem., 2011, 46, 3410-3419.

182 N. Zheng, P. Karra, M. A. Vandenberg, J. H. Kim, M. J. Webber, W. L. Holland and D. H. C. Chou, J. Med. Chem., 2019, 62, 11437-11443.

183 J. Tytgat, K. G. Chandy, M. L. Garcia, G. A. Gutman, M.-F. Martin-Eauclaire, J. J. van der Walt and L. D. Possani, Trends Pharmacol. Sci., 1999, 20, 444-447.

184 H. Juichi, R. Ando, T. Ishido, M. Miyashita, Y. Nakagawa and H. Miyagawa, J. Pept. Sci., 2018, 24, e3133.

185 H. Yajima, S. Funakoshi, N. Fujii, K. Akaji and H. Irie, Chem. Pharm. Bull., 1979, 27, 1060-1061.

186 A. R. Urmey and N. J. Zondlo, Pept. Sci., 2020, 112, e24137. 187 N. Fujii, A. Otaka, T. Watanabe, H. Arai, S. Funakoshi, K. Bessho and H. Yajima, J. Chem. Soc., Chem. Commun., 1987, 1676-1678.

188 F. Wu, J. P. Mayer, A. N. Zaykov, F. Zhang, F. Liu and R. D. DiMarchi, Chem. - Eur. J., 2016, 22, 9777-9783.

189 N. Fujii, A. Otaka, S. Funakoshi, T. Watanabe, H. Arai, K. Bessho and H. Yajima, J. Protein Chem., 1988, 7, 151-156.

190 N. Fujii, T. Watanabe, T. Aotake, A. Otaka, I. Yamamoto, J. Konishe and H. Yajima, Chem. Pharm. Bull., 1988, 36, 3304-3311.

191 V. J. Hruby, D. A. Upson and N. S. Agarwal, J. Org. Chem., 1977, 42, 3552-3556.

192 J. J. Knittel, T. K. Sawyer, V. J. Hruby and M. E. Hadley, J. Med. Chem., 1983, 26, 125-129.

193 S. Tsuda, T. Yoshiya, M. Mochizuki and Y. Nishiuchi, Org. Lett., 2015, 17, 1806-1809.

194 N. Fujii, H. Yajima, A. Otaka, S. Funakoshi, M. Nomizu, K. Akaji, I. Yamamoto, K. Torizuka, K. Kitagawa, T. Akita, K. Ando, T. Kawamoto, Y. Shimonishi and T. Takao, J. Chem. Soc., Chem. Commun., 1985, 602-603. 
195 S. Futaki, M. Aoki, T. Ishikawa, F. Kondo, T. Asahara, M. Niwa, Y. Nakaya, T. Yagami and K. Kitagawa, Bioorg. Med. Chem., 1999, 7, 187-192.

196 T. Brown, J. H. Jones and J. D. Richards, J. Chem. Soc., Perkin Trans. 1, 1982, 1553-1561.

197 T. Haack and M. Mutter, Tetrahedron Lett., 1992, 33, 1589-1592.

198 A. Kreżel, J. Wójcik, M. Maciejczyk and W. Bal, Inorg. Chem., 2011, 50, 72-85.

199 A. Krężel and W. Bal, Org. Biomol. Chem., 2003, 1, 3885-3890.

200 V. Martos, P. Castreño, M. Royo, F. Albericio and J. de Mendoza, J. Comb. Chem., 2009, 11, 410-421.

201 J. D. Smith, L. N. Cardwell, D. Porciani, A. Nolla, B. T. Cornelison, M. C. Schulte, F. Gallazzi, D. H. Burke, M. A. Daniels and B. D. Ulery, Mol. Syst. Des. Eng., 2020, 5, 269-283.

202 A. Aletras, K. Barlos, D. Gatos, S. Koutsogianni and P. Mamos, Int. J. Pept. Protein Res., 1995, 45, 488-496.

203 A. S. Galanis, F. Albericio and M. Grøtli, Pept. Sci., 2009, 92, 23-34.

204 A. Graziadio, M. Zanda, S. Frau, I. N. Fleming, M. Musolino, S. Dall'Angelo, M. Baldassarre and M. Piras, Bioconjugate Chem., 2016, 27, 1332-1340.

205 T. D. Kondasinghe, H. Y. Saraha, S. T. Jackowski and J. L. Stockdill, Tetrahedron Lett., 2019, 60, 23-28.

206 D.-L. Huang, C. Montigny, Y. Zheng, V. Beswick, Y. Li, X.-X. Cao, T. Barbot, C. Jaxel, J. Liang, M. Xue, C.-L. Tian, N. Jamin and J.-S. Zheng, Angew. Chem., Int. Ed., 2020, 59, 5178-5184.

207 D.-L. Huang, Y. Li, J. Liang, L. Yu, M. Xue, X.-X. Cao, B. Xiao, C.-L. Tian, L. Liu and J.-S. Zheng, J. Am. Chem. Soc., 2020, 142, 8790-8799.

208 J. Halstrøm, K. Brunfeldt and K. Kovács, Acta Chem. Scand., 1979, 33b, 685-689.

209 J. Tulla-Puche and G. Barany, J. Org. Chem., 2004, 69, 4101-4107.

210 S. Liu, B. L. Pentelute and S. B. H. Kent, Angew. Chem., Int. Ed., 2012, 51, 993-999.

211 H. Lamthanh, C. Roumestand, C. Deprun and A. Ménez, Int. J. Pept. Protein Res., 1993, 41, 85-95.

212 M. A. Y. Engebretsen, E. Agner, J. Sandosham and P. M. Fischer, J. Pept. Res., 1997, 49, 341-346.

213 Y. Wang, L. Han, N. Yuan, H. Wang, H. Li, J. Liu, H. Chen, Q. Zhang and S. Dong, Chem. Sci., 2018, 9, 1940-1946.

214 H. Chen, Y. Xiao, N. Yuan, J. Weng, P. Gao, L. Breindel, A. Shekhtman and Q. Zhang, Chem. Sci., 2018, 9, 1982-1988.

215 S. Laps, F. Atamleh, G. Kamnesky, H. Sun and A. Brik, Nat. Commun., 2021, 12, 870.

216 N. Fujii, A. Otaka, S. Funakoshi, K. Bessho and H. Yajima, J. Chem. Soc., Chem. Commun., 1987, 163-164.

217 M. Noda and M. Kiffe, J. Pept. Res., 1997, 50, 329-335.

218 A. K. Ivanov, E. É. Grigor'eva, A. A. Antonov and I. A. Donetskii, Chem. Nat. Compd., 1993, 29, 97-103.

219 M. Ueki, T. Inazu and S. Ikeda, Bull. Chem. Soc. Jpn., 1979, 52, 2424-2427.

220 K. Akaji, T. Tatsumi, M. Yoshida, T. Kimura, Y. Fujiwara and Y. Kiso, J. Am. Chem. Soc., 1992, 114, 4137-4143.
221 G. Greiner and P. Hermann, in Peptides 1990, Proceedings of the Twenty-First European Peptide Symposium, ed. E. Giralt and D. Andreu, ESCOM Science Publishers B.V., Leiden, 1991, pp. 227-278.

222 S. K. Maity, G. Mann, M. Jbara, S. Laps, G. Kamnesky and A. Brik, Org. Lett., 2016, 18, 3026-3029.

223 F. Liu, Q. Liu and A. R. Mezo, Org. Lett., 2014, 16, 3126-3129.

224 X. Yang, V. Gelfanov, F. Liu and R. DiMarchi, Org. Lett., 2016, 18, 5516-5519.

225 O. Dangles, F. Guibe, G. Balavoine, S. Lavielle and A. Marquet, J. Org. Chem., 1987, 52, 4984-4993.

226 F. Guibé, Tetrahedron, 1998, 54, 2967-3042.

227 A. Malanda Kimbonguila, A. Merzouk, F. Guibé and A. Loffet, Tetrahedron, 1999, 55, 6931-6944.

228 J. Borlinghaus, F. Albrecht, M. C. H. Gruhlke, I. D. Nwachukwu and A. J. Slusarenko, Molecules, 2014, 19, 12591-12618.

229 N. D. J. Yates, M. A. Fascione and A. Parkin, Chem. - Eur. J., 2018, 24, 12164-12182.

230 J. M. J. M. Ravasco, H. Faustino, A. Trindade and P. M. P. Gois, Chem. - Eur. J., 2019, 25, 43-59.

231 K. Renault, J. W. Fredy, P.-Y. Renard and C. Sabot, Bioconjugate Chem., 2018, 29, 2497-2513.

232 H. P. Hemantha, S. N. Bavikar, Y. Herman-Bachinsky, N. Haj-Yahya, S. Bondalapati, A. Ciechanover and A. Brik, J. Am. Chem. Soc., 2014, 136, 2665-2673.

233 M. Seenaiah, M. Jbara, S. M. Mali and A. Brik, Angew. Chem., Int. Ed., 2015, 54, 12374-12378.

234 V. Y. Torbeev and S. B. H. Kent, Angew. Chem., Int. Ed., 2007, 46, 1667-1670.

235 M. Jbara, S. K. Maity and A. Brik, Angew. Chem., Int. Ed., 2017, 56, 10644-10655.

236 G. Mann, G. Satish, R. Meledin, G. B. Vamisetti and A. Brik, Angew. Chem., Int. Ed., 2019, 58, 13540-13549.

237 R. L. Brabham, R. J. Spears, J. Walton, S. Tyagi, E. A. Lemke and M. A. Fascione, Chem. Commun., 2018, 54, 1501-1504.

238 X. Bi, K. K. Pasunooti, J. Lescar and C.-F. Liu, Bioconjugate Chem., 2017, 28, 325-329.

239 R. J. Spears, R. L. Brabham, D. Budhadev, T. Keenan, S. McKenna, J. Walton, J. A. Brannigan, A. M. Brzozowski, A. J. Wilkinson, M. Plevin and M. A. Fascione, Chem. Sci., 2018, 9, 5585-5593.

240 K. Ohkawachi, D. Kobayashi, K. Morimoto, A. Shigenaga, M. Denda, K. Yamatsugu, M. Kanai and A. Otaka, Org. Lett., 2020, 22, 5289-5293.

241 H. Katayama and K. Nagata, J. Pept. Sci., 2021, 27, e3290.

242 G. Prota and E. Ponsiglione, Tetrahedron, 1973, 29, 4271-4274.

243 G. J. Hoogerheidem, in Peptides 1980 (Proceedings of the sixteenth European peptide symposium), ed. K. Brunfeldt, Copenhagen, 1981.

244 J. O. Campeciño, L. W. Dudycz, D. Tumelty, V. Berg, D. E. Cabelli and M. J. Maroney, J. Am. Chem. Soc., 2015, 137, 9044-9052.

245 D. P. Nguyen, M. Mahesh, S. J. Elsässer, S. M. Hancock, C. Uttamapinant and J. W. Chin, J. Am. Chem. Soc., 2014, 136, 2240-2243.

246 N. Wu, A. Deiters, T. A. Cropp, D. King and P. G. Schultz, J. Am. Chem. Soc., 2004, 126, 14306-14307. 
247 K. D. Philipson, J. P. Gallivan, G. S. Brandt, D. A. Dougherty and H. A. Lester, Am. J. Physiol. - Cell Physiol., 2001, 281, C195-C206.

248 G. Marriott and M. Heidecker, Biochemistry, 1996, 35, 3170-3174.

249 A. G. Russell, M.-E. Ragoussi, R. Ramalho, C. W. Wharton, D. Carteau, D. M. Bassani and J. S. Snaith, J. Org. Chem., 2010, 75, 4648-4651.

250 S. P. Fodor, J. L. Read, M. C. Pirrung, L. Stryer, A. T. Lu and D. Solas, Science, 1991, 251, LP767-LP773.

251 A. Patchornik, B. Amit and R. B. Woodward, J. Am. Chem. Soc., 1970, 92, 6333-6335.

252 R. Miyajima, Y. Tsuda, T. Inokuma, A. Shigenaga, M. Imanishi, S. Futaki and A. Otaka, Biopolymers, 2016, 531-546.

253 R. Rakauskaitè, G. Urbanavičiūtè, A. Rukšènaitè, Z. Liutkevičiūtè, R. Juškėnas, V. Masevičius and S. Klimašauskas, Chem. Commun., 2015, 51, 8245-8248.

254 J. Pan and K. S. Carroll, Org. Lett., 2015, 17, 6014-6017.

255 J. H. Wosnick and M. S. Shoichet, Chem. Mater., 2008, 20, 55-60.

256 C. Berse, R. Boucher and L. Piché, J. Org. Chem., 1957, 22, 805-808.

257 M. D. Bachi and K. J. Ross-Petersen, J. Org. Chem., 1972, 37, 3550-3551.

258 S. J. Brois, J. F. Pilot and H. W. Barnum, J. Am. Chem. Soc., 1970, 92, 7629-7631.

259 L. Chen, I. Zoulíková, J. Slaninová and G. Barany, J. Med. Chem., 1997, 40, 864-876.

260 S. J. Mear and T. F. Jamison, J. Org. Chem., 2019, 84, 15001-15007.

261 R. Matsueda and K. Aiba, Chem. Lett., 1978, 951-952.

262 A. Taguchi, K. Fukumoto, Y. Asahina, A. Kajiyama, S. Shimura, K. Hamada, K. Takayama, F. Yakushiji, H. Hojo and Y. Hayashi, Org. Biomol. Chem., 2015, 13, 3186-3189.

263 Y. Cui, A. Taguchi, K. Kobayashi, H. Shida, K. Takayama, A. Taniguchi and Y. Hayashi, Org. Biomol. Chem., 2020, 18, 7094-7097.

264 E. J. Ste Marie, E. L. Ruggles and R. J. Hondal, J. Pept. Sci., 2016, 22, 571-576.

265 U. Weber and P. Hartter, Hoppe. Seylers. Z. Physiol. Chem, 1970, 351, 1384-1388.

266 P. S. Herradura, K. A. Pendola and R. K. Guy, Org. Lett., 2000, 2, 2019-2022.
267 S. S. More and R. Vince, Bioorg. Med. Chem. Lett., 2006, 16, 6039-6042.

268 L. Moroder, M. Gemeiner, W. Goehring, E. Jaeger, P. Thamm and E. Wünsch, Biopolymers, 1981, 20, 17-37.

269 S. Wang, D. Gao, K. Li, S. Ye, Q. Liu, Y. Peng, G. Lv, L. Qiu and J. Lin, Org. Biomol. Chem., 2020, 18, 3512-3521.

270 B. Roubinet, A. Chevalier, P.-Y. Renard and A. Romieu, Eur. J. Org. Chem., 2015, 166-182.

271 N. S. Khan, B. A. Riggle, G. K. Seward, Y. Bai and I. J. Dmochowski, Bioconjugate Chem., 2015, 26, 101-109.

272 C. Zhang, P. Dai, A. M. Spokoyny and B. L. Pentelute, Org. Lett., 2014, 16, 3652-3655.

273 J. A. Brailsford, J. L. Stockdill, A. J. Axelrod, M. T. Peterson, P. A. Vadola, E. V. Johnston and S. J. Danishefsky, Tetrahedron, 2018, 74, 1951-1956.

274 B. J. Umlauf, K. A. Mix, V. A. Grosskopf, R. T. Raines and E. V. Shusta, Bioconjugate Chem., 2018, 29, 1605-1613.

275 X. Zhao, G. Lv, K. Li, Y. Peng, Q. Liu, L. Qiu and J. Lin, Nucl. Med. Biol., 2020, 82-83, 72-79.

276 M. Góngora-Benítez, J. Tulla-Puche, M. Paradís-Bas, O. Werbitzky, M. Giraud and F. Albericio, Biopolymers, 2011, 96, 69-80.

277 G. Tang, T. Ji, A. F. Hu and Y. F. Zhao, Synlett, 2008, 1907-1909.

278 T. Kawakami, R. Yoshikawa, Y. Fujiyoshi, Y. Mishima, H. Hojo, S. Tajima and I. Suetake, J. Biochem., 2015, 158, 403-411.

279 P. Hartter, Biol. Chem., 1976, 357, 1683-1694.

280 T. M. Postma and F. Albericio, Org. Lett., 2013, 15, 616-619.

281 K. Krieger, B. Wängler, R. Schirrmacher and C. Wängler, ChemMedChem, 2020, 15, 1652-1660.

282 M. Ruiz-Gayo, F. Albericio, M. Pons, M. Royo, E. Pedroso and E. Giralt, Tetrahedron Lett., 1988, 29, 3845-3848.

283 H. Huang and R. I. Carey, J. Pept. Res., 1998, 51, 290-296.

284 R. A. D. Bathgate, F. Lin, N. F. Hanson, L. Otvos, A. Guidolin, C. Giannakis, S. Bastiras, S. L. Layfield, T. Ferraro, S. Ma, C. Zhao, A. L. Gundlach, C. S. Samuel, G. W. Tregear and J. D. Wade, Biochemistry, 2006, 45, 1043-1053.

285 D. E. K. Kabotso, D. Smiley, J. P. Mayer, V. M. Gelfanov, D. Perez-Tilve, R. D. DiMarchi, N. L. B. Pohl and F. Liu, J. Med. Chem., 2020, 63, 6134-6143.

286 O. Al Musaimi, B. G. de la Torre and F. Albericio, Green Chem., 2020, 22, 996-1018. 